\title{
Zwischenevaluierung der RIF 2000 Regionale Impulsförderung
}

im Auftrag des Bundesministeriums für Verkehr, Innovation und Technologie, Sektion III, Innovation und Telekommunikation, Abt. III/I1

Endbericht

Fritz Ohler Anton Geyer

November 2005

Technopolis Forschungs- und Beratungsgesellschaft mbH Prinz Eugen Straße 80/12

A 1040 Wien

Tel. +43 (1) 5039592 - 17

fritz.ohler@technopolis-group.com www.technopolis-group.com 


\section{Inhaltsverzeichnis}

1 Einleitung 1

2 Die Entstehung des RIF 2000 Programms 3

2.1 Errichtung von Impulszentren in Österreich seit 1986

2.2 Evaluierung der Regionalen Innovationsförderung Ende der 90er Jahre 7

2.3 Das neue RIF 2000 Programm: allgemeine und operationale Ziele 7

2.4 Der politische Kontext während der Entstehung des RIF 2000 Programms 9

2.5 Auf welche Probleme war RIF 2000 die Antwort? 11

3 Die Durchführung des RIF 2000 Programms 15

3.1 Vergleich der Abwicklungsprozeduren der beiden Förderungsmodule 15

3.2 Wahrnehmung und Handhabung des Ausschreibungsprinzips 17

4 Das REGplus Programm in Zahlen $\quad 20$

4.1 Geförderte Projekte, beantragte Förderungen, ausgeschüttete Förderungen 20

4.2 Angemessenheit der Projekte: Kürzungen und Auflagen 22

4.2.1 Kürzungen $\quad 22$

4.2.2 Auflagen bei Aufbau- und Erweiterungsprojekten 27

4.3 Trefferbilanz: Die Verteilung der Projekte und Fördermittel nach
Bundesländern

5 Der Entstehungskontext von RIF 2000 Projekten 42

5.1 Wie kommen RIF 2000 / REGplus Projekte zustande? 42

5.2 Inhaltlich-strategische Orientierung der geförderten Projekte 47

5.3 Projektportfolio der Zentren $\quad 55$

5.4 Das Netzwerk der österreichischen Technologie-, Impuls- und Gründerzentren $\begin{array}{ll}\text { und ihrer Dienstleistungen } & 63\end{array}$

6 Welche Rolle spielt die Entwicklungsagentur des Landes?

7 Woran kann man nach zwei, drei Jahren die Spuren des Projekts noch erkennen?

8 Lernen von Anderen: Eine Übersicht über die internationale Inkubatorszene

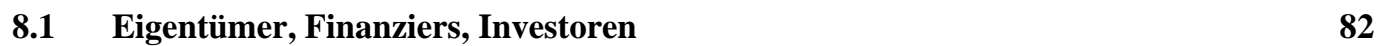

$\begin{array}{lll}\text { 8.2 Standorte und Größe von Impulszentren } & 83\end{array}$

8.3 Ziele, Rollen und strategischer Kontext von Impulszentren $\quad 84$

$\begin{array}{lll}8.4 & \text { Dienstleistungsportfolio von Impulszentren } & 84\end{array}$

8.5 Errichtungs- und Betriebskosten und deren Finanzierung, Erreichung des Break Even $\quad 85$

8.6 Abhängigkeit von öffentlicher Finanzierung $\quad 86$

8.7 Sind die österreichischen Impulszentren anders als die ausländischen
Inkubatoren?

9 Die Zukunft des RIF 2000 Programms $\quad 89$

$\begin{array}{lll}9.1 & \text { RIF } 2000 \text { Programm hat seine Mission erfüllt } & 89\end{array}$

$\begin{array}{lll}9.2 & \text { Kargheit und Fokussierung } & 90\end{array}$ 
9.3 Das Humanpotenzial nutzen

9.4 Hohe Anerkennung der Förderabwicklungsstelle und ihrer Mitarbeiter, veränderte Rolle in Zukunft

9.5 Ein neues Programm?

9.6 Checkliste für künftige Programmplanung aufgrund der Erfahrung mit RIF 2000: To whom it may concern! 


\section{Tabellenverzeichnis}

Tabelle 1 Entwicklung der F\&E-Ausgaben nach Finanzierungssektoren 3

Tabelle 2 Förder- und Entwicklungseinrichtungen der Länder und ihr Dienstleistungsportfolio 4

Tabelle 3 Verteilung der Impulszentren nach Bundesländern 6

Tabelle 4 Begriffsbestimmung: Impuls-, Technologie- und Gründerzentren 6

Tabelle 5 Allgemeine Ziele, operative Ziele und Kriteriensätze des RIF 2000 Programms

Tabelle 6 Veränderungen im institutionellen Umfeld seit Ende der 90er Jahre: Politik, Infrastrukturen, Finanzierung

Tabelle 7 Die Abwicklungsprozeduren der beiden Förderungsmodule im Vergleich

Tabelle 8 Ablauf des Ausschreibungsverfahrens im Fördermodul REGplus 17

Tabelle 9 Eingereichte, geförderte und abgelehnte Projekte im REGplus Programm

Tabelle 10

Ablehnungsquote bei ausgewählten Programmlinien

Tabelle 11 Förderintensität nach Runden

Tabelle 12

Kürzung des beantragten Fördervolmens nach Runde und Projekttyp 23

Tabelle 13

Kürzungen nach Runde und Projekttyp

Tabelle 14

Kürzungen bei Aufbau- und Erweiterungsprojekten

Tabelle 15

Auflagen an geförderte Projekte: Reduktionen, Präzisierungen, Vernetzungen

Tabelle 16

Abweichungen der Vorab-Bewertungen durch die Jury

Tabelle 17

Auflagen bei Aufbau- und Erweiterungsprojekten

31

Tabelle 18

Verteilung der REGplus Bundesförderung nach Bundesländern $\quad 36$

Tabelle 19

Projektkosten, Fördermittel und Trefferbilanz nach Bundesländer und

Ausschreibungsrunden

Tabelle 20 Motive und Umstände für das Zustandekommen von REGplus

Projekten

Tabelle 21 Orientierung der eingereichten / geförderten REGplus / RIFupgrade Projekte: Überblick

Tabelle 22

Orientierung der eingereichten / geförderten Projekte: detailliert

Tabelle 23 Projektportfolio von Förderwerbern im Fördermodul REGplus

Tabelle 24

Verflechtungen von Zentren und Projekten am Beispiel Ost- und Südsteiermark

Tabelle 25

Verteilung der Dienstleistungen in den Impulszentren

Tabelle 26

Technologie-, Impuls- und Gründerzentren in Österreich

Tabelle 27 Wirkungen und ausgebliebene Wirkungen der RIF 2000 Projekte 78

Tabelle 28 Schlüsselpartner von Impulszentren

Tabelle 29

Standorte von Impulszentren

Tabelle 30

Zahl der betreuten Unternehmen / Organisationen

Tabelle 31

Ziele von Impulszentren

Dienstleistungen von Impulszentren

Tabelle 33

Finanzierung der Errichtungskosten von Impulszentren

Tabelle 34

Finanzierung der Betriebskosten von Impulszentren

Tabelle 35

Konsequenzen des Rückzugs aus der öffentlichen Finanzierung von Impulszentren

Tabelle 36 Checkliste für künftige Programmplanung aufgrund der Erfahrung mit RIF 2000 


\section{Abbildungsverzeichnis}

Abbildung 1 Gründung von Impulszentren seit 1986

Abbildung 2 Größenverteilung der Impulszentren [Zahl der Mieter]

Abbildung 3 Größenverteilung der Impulszentren [vermietbare Fläche]

Abbildung 4 Verteilung der Dienstleistungen der Impulszentren 


\section{$1 \quad$ Einleitung}

Mit diesem Bericht wird die Zwischenevaluierung der 'RIF 2000 Regionale Impulsförderung' (im Folgenden: RIF 2000 oder RIF 2000 Programm) vorgelegt. Sie wurde im Auftrag des Bundesministeriums für Verkehr, Innovation und Technologie, Sektion III, Innovation und Telekommunikation, Abt. III/I1, durchgeführt.

Das RIF 2000 Programm ist die Fortsetzung früherer Förderaktivitäten des Bundesministerium für Verkehr, Innovation und Technologie bzw. (im Folgenden: bmvit) und seiner Vorgänger zur Förderung von Regionen, seiner Unternehmen, vor allem aber eines neueren Typs von regionalwirtschaftlichen Akteuren, den Technologie-, Gründer-, Innovations-, kurz: Impulszentren. Wir geben daher in Kapitel 2 einen Überblick über die Entstehung des RIF 2000 Programms und, damit verbunden, in die Entwicklung der Technologie- und Innovationspolitik der letzten 5-8 Jahre, also jenes Zeitraums, in dem das RIF 2000 Programm zum Thema wurde, konzipiert und schließlich umgesetzt worden ist. In diesem Zeitraum hat eine selten zuvor beobachtbare Dynamik stattgefunden. Sie beginnt mit dem Beitritt Österreichs zur EU und, damit verbunden, dem Zugang zu einschlägigen Förderungen. Sie setzt sich fort auf Ebene der Bundesländer, die zunehmend ihre eigene Technologie- und Innovationspolitik betreiben und endet vorläufig in einer weit reichenden Re-Organisation und Re-Orientierung der Politik auf Ebene des Bundes, namentlich der Einführung von Programmen, der Bildung neuer Agenturen (TIG, AWS, FFG) und, nicht zuletzt, der massiven Aufstockung der öffentlichen Mittel.

In Kapitel 3 wird die Durchführung des RIF 2000 Programms beschrieben, das in den Programmmodul RIFupgrade und REGplus aufgeteilt wurde. Diese beiden Module unterscheiden sich wesentlich darin, dass ersteres dem Antragsprinzip folgt, bei dem Förderanträge jederzeit eingereicht werden können, wohingegen das Programmmodul REGplus dem damals neu eingeführten Prinzip der Programmausschreibung folgt. Die zentrale Evaluierungsfrage ist hier, inwieweit dieses neue Prinzip von den Förderadressaten angenommen und von der Förderungsabwicklungsstelle gehandhabt wurde.

In Kapitel 4 geben wir einen Überblick über das Programmmodul REGplus. Hierfür hatten wir Zugang zu den von der Förderungsabwicklungsstelle (Technologie Impulse Gesellschaft, nunmehr Bereich 2 der Forschungsförderungsgesellschaft, FFG), gesammelten Informationen. Neben einer Analyse der geförderten Projekte unter dem Gesichtspunkt der beantragten bzw. ausgeschütteten Förderungen hat uns das uns zur Verfügung gestellte Daten- und Informationsmaterial erlaubt, eine detaillierte Analyse über die 'Angemessenheit der Projekte' bzw. des gesamten Programms durchzuführen. Aus der Analyse der Inhalte und Häufigkeiten der Begründungen für Kürzungen und Auflagen konnten wir Rückschlüsse darüber gewinnen, inwieweit REGplus in Bezug auf seine Zielsetzungen und seine Qualitäten bei den unterschiedlichen, mit Inhalt und Qualität befassten Akteuren (Förderungswerber, Förderungsabwicklungsstelle, Jury) verstanden, kommuniziert und darauf reagiert wurde. Schließlich, weil die RIF 2000 Projekte grundsätzlich der Ko-Finanzierung durch die Länder bedürfen, wurde eine Analyse nach Bundesländern durchgeführt, weil es die begründete Annahme gab, wonach die Politik der jeweiligen Bundesländer einen nicht unerheblichen Einfluss auf Umfang und Qualität der Anträge haben würde. 
Kapitel 5 beleuchtet den Entstehungskontext von RIF 2000 Projekten und stellt insbesondere die Projekte aus dem RIF 2000 Programm in den Kontext anderer, von den Förderempfängern eingeworbenen bzw. durchgeführten Projekte. Hintergrundannahme war hier, dass es einen wesentlichen Unterschied für die Relevanz des RIF 2000 Programms machen würde, wenn die Förderadressaten überwiegend oder gar ausschließlich auf Projekte des RIF 2000 Programm zurückgreifen würden oder ob es hier eine breitere Palette von Fördermöglichkeiten, ja sogar Wahlmöglichkeiten geben würde.

Weil, wie bereits erwähnt, Förderungen des Bundes nur gewährt wurden, wenn auch die Länder die Ko-Finanzierung übernehmen würden und hier von Seiten der Länder eine mehr oder weniger aktive Politik zu erwarten war, wird dieser Frage nachgegangen, u.zw. in Kapitel 6.

Obgleich es sich um eine Zwischenevaluierung handelt, darf die Frage nach den Wirkungen gestellt werden, zumal dann, wenn ein nicht unerheblicher Teil der Projekte bereits abgeschlossen ist. Wir sind dieser Frage in Kapitel 7 nachgegangen und es mit "Woran kann man nach zwei, drei Jahren die Spuren des Projekts noch erkennen?" übertitelt.

Evaluierungen, zumal Zwischenevaluierungen dienen dazu, um zu lernen und um das noch laufende Programm anzupassen und zu verbessern, zumindest um andere, vergleichbare Programme besser zu gestalten und abzuwickeln. Dies schließt folgerichtig ein, von Anderen zu lernen. Wir tun dies in Kapitel 8, wo wir auf eine der wenigen, und unter Aktualitätsgesichtspunkten einzigen Benchmarking-Studie zurückgreifen, diese in ihren wichtigsten Ergebnissen referieren und unsere Beobachtungen aus der detaillieren Kenntnis der österreichischen Verhältnisse daneben stellen und vergleichen.

Schließlich ziehen wir in Kapitel 9 Schlüsse für die Zukunft des RIF 2000 Programms, das, es sei vorweggenommen, seine wesentlichen Ziele erreicht und seine Mission insoweit erfüllt hat. Unter dem Titel "To whom it may concern!" schließen wir den Bericht mit einer Auflistung von Beobachtungen ab, die wert sind, bei der künftigen Planung von ähnlichen Programmen berücksichtigt zu werden. 


\section{Die Entstehung des RIF 2000 Programms}

Impulszentren $^{1}$ als Instrument von Industrie-, Innovations- und Regionalpolitik ist ein Phänomen, das sowohl in Österreich als auch international betrachtet, ein typisches Phänomen der 90er Jahre ist. Die 90er Jahre sind umgekehrt auch das Jahrzehnt, in dem Innovationspolitik seine Hochblüte erfahren hat. Sie war angesiedelt zwischen Industriepolitik einerseits und Forschungs- und Technologiepolitik andererseits. Zudem hat man verstärkt die KMUs als Träger von Innovation und technologischem Wandel entdeckt. Nicht zuletzt waren die Leitkonzepte der 90er Jahre 'Innovationssystem' und 'Cluster', in denen all diese Konzepte und Politikzugänge ihre konzeptuellen Grundlagen haben. Beide weisen einen gemeinsamen Kern darin auf, dass die Innovationsleistung einer Nation bzw. Region wesentlich vom Wechselspiel der Unternehmen untereinander sowie der Spezialisierung einschlägiger Institutionen (Forschung, Aus- und Weiterbildung, Regulierung, öffentliche Verwaltung) geprägt wird. Dementsprechend kann auch das Konzept der Vernetzung als der wohl prägendste Politikansatz der 90er Jahre bezeichnet werden.

Ein zweites, beachtenswertes Phänomen der, nunmehr späten, 90er Jahre ist das Auftauchen der Bundesländer als wesentlicher technologie- und innovationspolitischer Akteur. So haben sich im Zeitraum 1998-2005 die Aufwendungen des Bundes für F\&E um 58\% erhöht. Die der Länder sind dagegen im Ausmaß von 131\% gestiegen (vgl. Tabelle 1).

Tabelle 1 Entwicklung der F\&E-Ausgaben nach Finanzierungssektoren

\begin{tabular}{|l|r|r|r|r|r|r|r|r|}
\hline \multicolumn{1}{|c|}{ Finanzierungssektoren } & $\mathbf{1 9 9 8}$ & $\mathbf{1 9 9 9}$ & $\mathbf{2 0 0 0}$ & $\mathbf{2 0 0 1}$ & $\mathbf{2 0 0 2}$ & $\mathbf{2 0 0 3}$ & $\mathbf{2 0 0 4}$ & $\mathbf{2 0 0 5}$ \\
\hline $\begin{array}{l}\text { Bruttoinlandsausgaben für } \\
\text { F\&E [Mio. EUR] }\end{array}$ & 3.400 & 3.762 & 4.029 & 4.393 & 4.788 & 4.902 & 5.274 & 5.773 \\
\hline Davon finanziert durch & & & & & & & & \\
\hline Bund & 1.098 & 1.201 & 1.225 & 1.351 & 1.408 & 1.360 & 1.579 & 1.741 \\
\hline Bundesländer & 142 & 206 & 249 & 280 & 274 & 301 & 302 & 329 \\
\hline Unternehmenssektor & 1.418 & 1.545 & 1.684 & 1.835 & 1.999 & 2.088 & 2.188 & 2.480 \\
\hline Ausland & 685 & 739 & 800 & 863 & 1.041 & 1.085 & 1.134 & 1.159 \\
\hline Sonstige & 57 & 71 & 70 & 64 & 66 & 68 & 70 & 64 \\
\hline BIP nominell [Mrd. EUR] & 191 & 197 & 207 & 213 & 218 & 224 & 232 & 245 \\
\hline $\begin{array}{l}\text { Bruttoinlandsausgaben für } \\
\text { F\&E [\% des BIP] }\end{array}$ & 1,78 & 1,91 & 1,95 & 2,07 & 2,19 & 2,19 & 2,27 & 2,35 \\
\hline
\end{tabular}

Quelle: STATISTIK AUSTRIA, Forschungs- und Technologieberichte der Bundesregierung http://portal.wko.at/portal.wk?AngID=1\&CtxID=16\&DstID=0\&subcontentparam=AngID\%3d1

Zu dieser Erhöhung der Mittel der Bundesländer kommt noch hinzu, dass die Länder einen deutlich höheren Anteil an disponiblen Mitteln aufweisen, die sie für ein breites Portfolio von Dienstleistungen einsetzen. Tabelle 2 liefert hierzu eine Übersicht, die im Wesentlichen zeigt, dass in den meisten Bundesländern das Portfolio reich bestückt ist.

1 'Impulszentren' wird in diesem Bericht als Sammelbegriff verwendet. Für eine Begriffsdefinition, die dem RIF 2000 zugrundeliegt, vgl. Tabelle 4. 
Tabelle 2 Förder- und Entwicklungseinrichtungen der Länder und ihr Dienstleistungsportfolio

\begin{tabular}{|c|c|c|c|c|c|c|c|c|c|}
\hline Bundesland & Einrichtung & 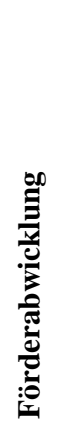 & 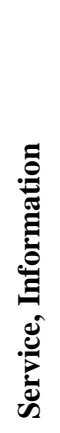 & 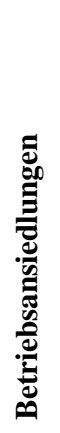 & 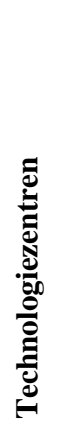 & 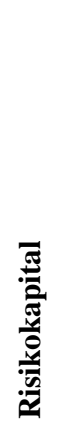 & 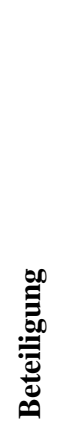 & 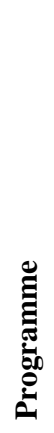 & 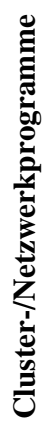 \\
\hline Burgenland & $\begin{array}{l}\text { Wirtschaftsservice Burgenland } \\
\text { Aktiengesellschaft (WIBAG) }\end{array}$ & $\mathrm{x}$ & $\mathrm{x}$ & $\mathrm{x}$ & $\mathrm{x}$ & $\mathrm{x}$ & $\mathrm{x}$ & $\mathrm{x}$ & $\mathrm{x}$ \\
\hline \multirow[t]{3}{*}{ Kärnten } & Kärntner Wirtschaftsförderungsfond (KWF) & $\mathrm{x}$ & & & $\mathrm{x}$ & & $\mathrm{x}$ & $\mathrm{x}$ & $\mathrm{x}$ \\
\hline & $\begin{array}{l}\text { Kärntner Betriebsansiedlungs- und } \\
\text { Beteiligungsgesellschaft (BABEG) }\end{array}$ & $\mathrm{x}$ & $\mathrm{x}$ & $\mathrm{x}$ & $\mathrm{x}$ & $\mathrm{x}$ & $\mathrm{x}$ & & \\
\hline & Entwicklungsagentur Kärnten (EAK) & & $\mathrm{x}$ & $\mathrm{x}$ & $\mathrm{x}$ & & $\mathrm{x}$ & & $\mathrm{x}$ \\
\hline \multirow[t]{4}{*}{ Niederösterreich } & ecoplus & $\mathrm{x}$ & $\mathrm{x}$ & $\mathrm{x}$ & $\mathrm{x}$ & & $\mathrm{x}$ & $\mathrm{x}$ & $\mathrm{x}$ \\
\hline & TecNet Company & & & & & $\mathrm{x}$ & $\mathrm{x}$ & & \\
\hline & Regionales Innovationszentrum (RIZ) & & $\mathrm{x}$ & & $\mathrm{x}$ & & & & \\
\hline & Land NÖ, Abteilung Wirtschaftsförderung & $\mathrm{x}$ & $\mathrm{x}$ & & & & & $\mathrm{x}$ & $\mathrm{x}$ \\
\hline \multirow[t]{2}{*}{ Oberösterreich } & $\begin{array}{l}\text { OÖ Technologie Marketing Gesellschaft } \\
\text { (TMG) }\end{array}$ & & $\mathrm{x}$ & $\mathrm{x}$ & $\mathrm{x}$ & & $\mathrm{x}$ & $\mathrm{x}$ & $\mathrm{x}$ \\
\hline & Land Oberösterreich, Abteilung Gewerbe & $\mathrm{x}$ & & & & & & & \\
\hline \multirow[t]{3}{*}{ Salzburg } & SalzburgAgentur & & $\mathrm{x}$ & $\mathrm{x}$ & & & & & \\
\hline & $\begin{array}{l}\text { Innovations- und Technologietransfer } \\
\text { Gesellschaft (ITG) } \\
\end{array}$ & & $\mathrm{x}$ & & & & & & $\mathrm{x}$ \\
\hline & $\begin{array}{l}\text { Land Salzburg, Abteilung 15: Wirtschaft, } \\
\text { Tourismus und Energie }\end{array}$ & $\mathrm{x}$ & & & & & & & \\
\hline \multirow[t]{2}{*}{ Steiermark } & $\begin{array}{l}\text { Steirische Wirtschaftsförderungsgesellschaft } \\
\text { (SFG) }\end{array}$ & $\mathrm{x}$ & $\mathrm{x}$ & $\mathrm{x}$ & $\mathrm{x}$ & $\mathrm{x}$ & $\mathrm{x}$ & $\mathrm{x}$ & $\mathrm{x}$ \\
\hline & $\begin{array}{l}\text { Amt der Steiermärkischen Landesregierung, } \\
\text { Abteilung } 3 \text { - Wissenschaft und Forschung }\end{array}$ & $\mathrm{x}$ & & & & & $\mathrm{x}$ & $\mathrm{x}$ & \\
\hline \multirow[t]{2}{*}{ Tirol } & Tiroler Zukunftsstiftung (TZS) & $\mathrm{x}$ & $\mathrm{x}$ & $\mathrm{x}$ & $\mathrm{x}$ & & $\mathrm{x}$ & $\mathrm{x}$ & $\mathrm{x}$ \\
\hline & Abteilung Wirtschaft und Arbeit & $\mathrm{x}$ & $\mathrm{x}$ & & $\mathrm{x}$ & & & $\mathrm{x}$ & \\
\hline \multirow[t]{2}{*}{ Vorarlberg } & $\begin{array}{l}\text { Wirtschafts-Standort Vorarlberg GmbH } \\
\text { (WISTO) }\end{array}$ & & $\mathrm{x}$ & $\mathrm{x}$ & $\mathrm{x}$ & $\mathrm{x}$ & & & \\
\hline & $\begin{array}{l}\text { Land Vorarlberg, Abteilung für Allgemeine } \\
\text { Wirtschaftsangelegenheiten }\end{array}$ & $\mathrm{x}$ & & & & & & & \\
\hline \multirow[t]{3}{*}{ Wien } & Wiener Wirtschaftsförderungsfond (WWFF) & $\mathrm{x}$ & $\mathrm{x}$ & $\mathrm{x}$ & & & $\mathrm{x}$ & $\mathrm{x}$ & \multirow{3}{*}{$\mathrm{x}^{\mathrm{a}}$} \\
\hline & $\begin{array}{l}\begin{array}{l}\text { Zentrum für Innovation und Technologie } \\
\text { (ZIT) }\end{array} \\
\end{array}$ & $\mathrm{x}$ & & & $\mathrm{x}$ & & $\mathrm{x}$ & $\mathrm{x}$ & \\
\hline & $\begin{array}{l}\text { Wiener Wissenschafts-, Forschungs- und } \\
\text { Technologiefonds (WWTF) }\end{array}$ & $\mathrm{X}$ & & & & & & $\mathrm{x}$ & \\
\hline Summe & & 14 & 12 & 10 & 11 & 5 & 11 & 9 & 9 \\
\hline
\end{tabular}

Quelle: Ohler, F., Neue Wege in der Forschungs-, Technologie- und Innovationspolitik zwischen Bund und

Bundesländer. Studie im Auftrag des Rats für Forschung und Technologieentwicklung, Technopolis. Wien 2004.

${ }^{a}$ : Wien hat keine Clusterprogramme in dem Sinn, wie sie etwa Oberösterreich oder Niederösterreich betreiben.

Betrachtet man aber die Aktivitäten der hier angeführten Agenturen, so kann man feststellen, dass mit unter-

schiedlichen Instrumenten und Programmen dieselben Zielgruppen und Themen von unterschiedlicher Seite, aber aufeinander abgestimmt, angesprochen werden. 


\subsection{Errichtung von Impulszentren ${ }^{2}$ in Österreich seit 1986}

Abbildung 1 zeigt, wie die Entwicklung von Impulszentren in Österreich verlaufen ist. Abgesehen von ein paar versprengten Zentren in den späten 80er und frühen 90er Jahren, in denen die Steiermark und Salzburg die Pioniere sind, boomt das Konzept der Impulszentren erst ab 1998 bzw., wenn mit die Vorbereitung einrechnet, ab Mitte der 90er Jahre. Diese Phase koinzidiert auch mit dem verstärkten Auftauchen der Bundesländer als technologie- und innovationspolitischer Akteur und indirekt die Mitgliedschaft in der EU und damit verbunden, die Inanspruchnahme von Regionalfördermitteln.

Abbildung 1 Gründung von Impulszentren seit 1986

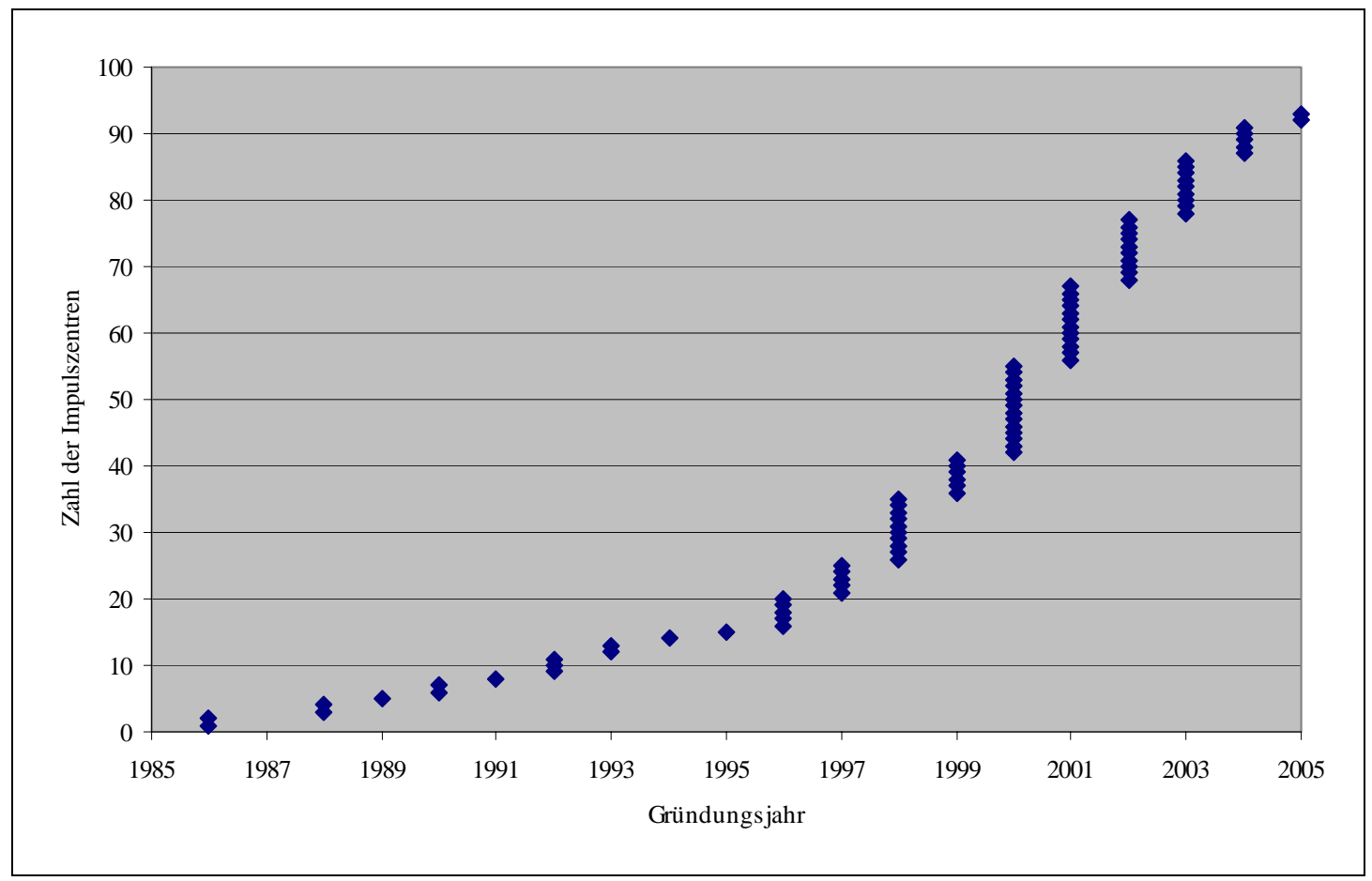

Quelle: www.vto.at sowie die jeweiligen Homepages

Tabelle 3 zeigt die Verteilung der bislang errichteten Zentren bezogen auf ihren Standort nach Bundesländern. Zunächst ist die Zahl selbst im internationalen Vergleich sehr hoch. Sodann fällt auf, dass die Verteilung in mancherlei Hinsicht wenig überraschend ist, in anderer Hinsicht sehr wohl. Dass die Steiermark und Oberösterreich die Liste anführen, ist ein Phänomen, dass wir weiter unten noch einmal vorfinden werden ${ }^{3}$ und das auch bei anderen Programmen vorzufinden ist, namentlich bei den Kompetenzzentrenprogrammen. Den Ausreißer stellt Niederösterreich dar, das in Bezug auf Impulszentren eine durchaus expansive Politik verfolgt hat, was angesichts seines Flächencharakters bei gleichzeitiger Abwesenheit von industriellen Zentren durchaus konsistent ist. Der Flächencharakter trifft überdies auch für die Steiermark und Oberösterreich zu, so dass die Vermutung plausibel ist, dass Technologiezentren auch als Instrument zum Ausgleich regionaler Disparitäten betrachtet wurden und werden. Dort, wo Gelder aus der Regionalförderung der EU in Anspruch

2 Der Begriff 'Impulszentren' wird hier als Sammelbegriff verwendet. Für eine Definition vgl. Tabelle 4.

3 Vgl. Tabelle 18 bzw. Kapitel 4.3. 
genommen wurden, war und ist die Überwindung regionaler Disparitäten sogar ausdrückliches Programm.

Tabelle 3 Verteilung der Impulszentren nach Bundesländern

\begin{tabular}{|l|c|c|c|}
\hline & Zahl der Impulszentren & Anteil [\%] & Anteil [\%kum] \\
\hline Steiermark & 23 & $22 \%$ & $22 \%$ \\
\hline Oberösterreich & 19 & $18 \%$ & $41 \%$ \\
\hline Niederösterreich & 17 & $17 \%$ & $57 \%$ \\
\hline Kärnten & 8 & $8 \%$ & $65 \%$ \\
\hline Tirol & 8 & $8 \%$ & $73 \%$ \\
\hline Vorarlberg & 8 & $8 \%$ & $81 \%$ \\
\hline Salzburg & 7 & $7 \%$ & $87 \%$ \\
\hline Wien & 7 & $7 \%$ & $94 \%$ \\
\hline Burgenland & 6 & $6 \%$ & $100 \%$ \\
\hline Gesamt & $\mathbf{1 0 3}$ & & \\
\hline
\end{tabular}

Quelle: www.vto.at

Eine genauere Betrachtung zeigt übrigens, dass der Bund viel früher die Bedeutung von Impulszentren erkannt hat. Zahlreiche Gründungen in den späten 80er und frühen 90er Jahre, vor allem in entwicklungsschwachen bzw. krisenhaften Regionen, sind auf die starke Präsenz des Bundes zurückzuführen.

Tabelle 4 Begriffsbestimmung: Impuls-, Technologie- und Gründerzentren

\begin{tabular}{|l|l|}
\hline Impulszentren & $\begin{array}{l}\text { Der Sammelbegriff Impulszentrum umfasst im engeren Sinn - unabhängig von } \\
\text { der Finanzierungs- und Eigentümerstruktur - Zentren mit regionsbezogenen, } \\
\text { regionalwirtschaftlichen Aufgaben, von denen in der Regel auch Initiativen zur } \\
\text { Verbesserung regionaler Innovationssysteme ausgehen. In solchen Impuls- } \\
\text { zentren werden einer konzentriert angesiedelten Standortgemeinschaft von } \\
\text { Unternehmen, deren betriebliche Tätigkeit vorwiegend in der Entwicklung, } \\
\text { Produktion und Vermarktung neuer Produkte, Verfahren und Dienstleistungen } \\
\text { besteht, gemeinsame hochwertige infrastrukturelle Einrichtungen sowie } \\
\text { Dienstleistungen durch ein Zentrums-Management angeboten. }\end{array}$ \\
& $\begin{array}{l}\text { Im weiteren Sinn sind dem Sammelbegriff Impulszentrum auch so genannte } \\
\text { 'virtuelle Impulszentren' zuzuordnen, das sind Zentren mit regionsbezogenen, } \\
\text { regionalwirtschaftlichen Aufgaben ohne materielle Infrastruktur. Impulszentren } \\
\text { im Sinne dieser Sonderrichtlinien umfassen somit Gründer- und Technologie- } \\
\text { zentren, Technologietransferzentren, Innovationszentren sowie im weiteren Sinn } \\
\text { auch „virtuelle Impulszentren“. } \\
\text { Vom Begriff Impulszentrum nicht umfasst sind reine Immobilienprojekte, d.h. } \\
\text { Büro-, Labor- oder Produktionsräumlichkeiten ohne gemeinsames Management } \\
\text { und als Standortgemeinschaft ohne Verflechtungen und ohne gemeinsame } \\
\text { regionsbezogene wirtschaftliche oder sozioökonomische Ziele. Weiters fallen } \\
\text { nicht unter den Begriff Impulszentrum regionale Agenturen, Landesförderungs- } \\
\text { stellen oder vergleichbare Organisationen. }\end{array}$ \\
\hline
\end{tabular}




\begin{tabular}{|c|c|}
\hline Technologiezentren & $\begin{array}{l}\text { Die Zielsetzung von Technologiezentren besteht im wesentlichen darin, den } \\
\text { Technologietransfer zwischen Wissenschaft und Wirtschaft zu fördern und neue } \\
\text { hoch qualifizierte Arbeitsplätze in technologieorientierten Unternehmen zu } \\
\text { schaffen. Technologiezentren sind daher Standortgemeinschaften überwiegend } \\
\text { junger Unternehmen, die technologisch neue Produkte und Verfahren } \\
\text { entwickeln und vermarkten. Für deren Entwicklung sind nicht nur Service- und } \\
\text { Beratungseinrichtungen, sondern auch ein regional bedeutsames Netzwerk von } \\
\text { Forschungs- und Finanzierungsinstitutionen wichtige Rahmenbedingungen. Die } \\
\text { F\&E-Komponente steht in Technologiezentren im Vordergrund und ist meistens } \\
\text { auch auf bestimmte Technologiethemen ausgerichtet. Zielgruppen sind daher } \\
\text { überwiegend Hochtechnologiefirmen, welche für die ersten Jahre nach der } \\
\text { Gründung Unterkunft, Beratung, Förderung und Dienstleistungen erhalten. Die } \\
\text { Verweildauer ist zum Teil, unabhängig vom Erfolg der Unternehmungen, } \\
\text { begrenzt. }\end{array}$ \\
\hline Grü & $\begin{array}{l}\text { Gründerzentren entsprechen weitgehend den Zielsetzungen und dem Leistungs- } \\
\text { angebot der Technologiezentren. Allerdings wenden sich Gründerzentren eher } \\
\text { an Unternehmen des verarbeitenden Gewerbes und der produktionsorientierten } \\
\text { Dienstleistungen, deren Wachstums- bzw. Überlebenschancen in der Startphase } \\
\text { durch Bereitstellung von Infrastruktur und Dienstleistungen durch die Träger- } \\
\text { gesellschaft erhöht werden sollen. Je nach individueller Ausrichtung eines Grün- } \\
\text { derzentrums kann aber auch die Technologieorientierung bzw. der Technologie- } \\
\text { schwerpunkt der Neugründer ein Kriterium bei der Auswahl der Unternehmen } \\
\text { darstellen. Die Verweildauer der Mieterunternehmen in einem Gründerzentrum } \\
\text { ist in der Regel zeitlich beschränkt. Innovationszentren Ein Innovationszentrum } \\
\text { ist eine Standortgemeinschaft von vorwiegend jungen Betrieben, die auf techno- } \\
\text { logisch hohem Niveau produzieren, neue Produkte und/oder neue Verfahren } \\
\text { entwickeln und vermarkten, sowie gemeinsam mit dem Zentrumsmanagement } \\
\text { Kooperations- und Netzwerkprojekte initiieren. Das Zentrumsmanagement } \\
\text { erfüllt darüber hinaus meist Dienstleistungs- und Beratungsfunktionen für die } \\
\text { Entwicklung der Region. }\end{array}$ \\
\hline
\end{tabular}

Quelle: Technologie Impulse Gesellschaft, Sonderrichtlinien RIF 2000 - Regionale Impulsförderung, bmvit / TIG, Wien, 2002

\subsection{Evaluierung der Regionalen Innovationsförderung Ende der 90er Jahre}

Ende der 90er Jahre wurde eine Evaluierung des damaligen RIF-Programms durchgeführt, die in Bezug auf die Impulszentren im Wesentlichen folgendes Ergebnis zutage förderte: Zwar sind die Unternehmen in den Impulszentren in technologiebzw. know-how intensiveren Sektoren angesiedelt, allerdings bleiben sie hinter den Erwartungen in Bezug auf die zwei wichtigsten Zielsetzungen von Impulszentren, nämlich eine Inkubator- bzw. Treibhausfunktion zu gewährleisten, also (i) höhere Überlebensrate bei jungen Unternehmen und (ii) rascheres Wachstum, jeweils verglichen mit Unternehmen außerhalb der Zentren. Beide Funktionen wurden nicht befriedigend erfüllt, ganz zu schweigen davon, dass die Impulszentren ihre Dienstleistungen auch an Dritte außerhalb der Zentren nachhaltig angeboten hätten.

\subsection{Das neue RIF 2000 Programm: allgemeine und operationale Ziele}

Aufgrund der Ergebnisse der Evaluierung der Regionalen Innovationsförderung wurde in den Jahren 1999-2000 ein neues Förderkonzept ausgearbeitet, das nicht nur auf diese beiden Funktionen abstellen sollte. Darüber hinaus sollten von den Zentren auch Impulse (daher auch der Name 'Impulszentren') für die Entwicklung der Region ausgehen. Tabelle 5 gibt eine Übersicht über die allgemeinen und operativen Ziele und Kriteriensätze des RIF 2000 Programms. Darin fallen folgende Punkte besonders auf: 
- Der Gedanke des Innovationssystems steht deutlich im Vordergrund. Die Impulszentren werden verstanden als (lokale) Entwicklungsagenturen, die ihre Ressourcen durch Kooperation mit anderen Impulszentren sowie mit einschlägigen Bildungs-, Forschungs- und Infrastruktureinrichtungen auf regionaler, nationaler und internationaler Basis erschließen.

- Die Leistungsfähigkeit dieser Agenturfunktion wird auf große Ziele hin ausgerichtet: Stärkung der Innovationskraft insb. klein- und mittelbetrieblicher Unternehmensstrukturen in den Regionen durch die Verbreiterung der Wissensbasis und durch Technologietransfer. Beiträge zur Verbesserung sozioökonomischer Parameter wie Beschäftigungsquote, berufliche Qualifikation und Mobilität, Verringerung der Abwanderung, Gründerdynamik. Eine kritische Betrachtung der allgemeinen Ziele führt zu der Einsicht, dass diese nur mäßig diskriminieren, insofern sich zahlreiche anders konzipierte Programme und Maßnahmen denken lassen, die diese Ziele auch oder sogar noch besser zu erfüllen imstande sind. Wir relativieren diese Kritik aber letztlich dadurch, dass es durchaus legitim, ja sogar notwendig ist, einen größeren Zielhorizont vorzugeben. Die Notwendigkeit ergibt sich nicht zuletzt dadurch, dass die großen Ziele ${ }^{4}$ die konzeptuelle Grundlage für Politikkoordination darstellen.

- Während also die allgemeinen Ziele für eine Vielzahl von Programmen gelten können, sind die operativen Ziele ausschließlich auf Impulszentren gerichtet, die entweder durch eigene Dienstleistungen bzw. vorgehaltene Infrastrukturen die Leistungsfähigkeit des regionalen Innovationssystems erhöhen sollen oder Dritte darin unterstützen. Ein wesentliches gemeinsames Charakteristikum der operativen Ziele ist die Aufwertung der Managementkapazitäten und -fähigkeiten sowie des Wissensstandes unter den beteiligten Akteuren. Sogar das Ziel 'Schließung von Lücken regionaler Innovationssysteme und Infrastrukturen durch Schaffung 'virtueller Impulszentren' in Form dauerhafter, projektorientierter Trägerstrukturen für regionale Innovationsvorhaben' stellt wesentlich auf die Fähigkeit ab, solche Trägerstrukturen aufzubauen und aufrecht zu erhalten. Wir sehen hier einen starken Fokus auf Humanpotenzial, Beziehungen, Wissen und Orientierung ${ }^{5}$ und damit auf wesentliche Elemente des gegenwärtigen Verständnisses von Innovation und Innovationsprozessen.

- Von Infrastrukturen i.e.S. ist keine Rede mehr. Ausgenommen davon die damals vorherrschende Überbewertung von Informations- und Kommunikationstechnologien. Damit verbunden ist auch die Idee 'virtueller Impulszentren', namentlich in Form von dauerhaften, projektorientierten Trägerstrukturen für regionale Innovationsvorhaben - auch ein Kind ihrer Zeit.

- Zusammenfassend lässt sich sagen, das damals eine deutliche Kehrtwendung vollzogen wurde: Abkehr von der Orientierung auf (physische) Infrastruktur,

$4 \quad$ Unter dem Titel 'Ziele' sind teilweise auch Strategien enthalten, wenn es etwa heißt "Stärkung der Innovationskraft insb. klein- und mittelbetrieblicher Unternehmensstrukturen in den Regionen durch die Verbreiterung der Wissensbasis und durch Technologietransfer". [Hervorhebung durch die Autoren]

5 Aus der Evaluierung diverser Programme mit einem hohen Gehalt an Kooperationsprojekten wissen wir, dass oftmals das vorgebliche materielle Ergebnis gar nicht im Vordergrund steht. Vielmehr geht es häufig um den Aufbau von Vertrauen, gemeinsamer Zielsetzungen und Werte, Erproben von Arbeitsbeziehungen usw.. Dementsprechend ist die Fortsetzung der Beziehungen trotz mäßigem Ausgang des Primärvorhabens oft das eigentlich erfreuliche Ergebnis. 
Hinwendung zum Konzept des Innovationssystems, angewandt auf Regionen. Generell herrscht ein großer Optimismus, der sich auch konzeptuell darin ausdrückt, dass man auf regionaler Ebene, d.h. hier im Einzugsbereich von Impulszentren, ein einigermaßen komplettes und leistungsfähiges Innovationssystem, zumindest virtuell, nachbilden kann. Managementkapazitäten und -fähigkeiten, vor allem im Aufbau von Beziehungsnetzwerken zwischen Unternehmen bzw. mit einschlägigen Institutionen wird eine hohe Bedeutung und Wertschätzung eingeräumt.

Tabelle 5 Allgemeine Ziele, operative Ziele und Kriteriensätze des RIF 2000 Programms

\begin{tabular}{|c|c|c|}
\hline Allgemeine Ziele & Operative Ziele & Kriteriensätze \\
\hline $\begin{array}{l}\text { Verbesserung des Innovations- } \\
\text { systems österreichischer Regio- } \\
\text { nen bezogen auf die jeweilige } \\
\text { Ausgangslage. Leitvorstellung des } \\
\text { Förderungsprogramms ist die } \\
\text { 'Innovative Region'. } \\
\text { Stärkung der Innovationskraft } \\
\text { insb. klein- und mittelbetrieblicher } \\
\text { Unternehmensstrukturen in den } \\
\text { Regionen durch die Verbreiterung } \\
\text { der Wissensbasis und durch } \\
\text { Technologietransfer. } \\
\text { Beiträge zur Verbesserung } \\
\text { sozioökonomischer Parameter } \\
\text { wie Beschäftigungsquote, } \\
\text { berufliche Qualifikation und } \\
\text { Mobilität, Verringerung der } \\
\text { Abwanderung, Gründerdynamik. }\end{array}$ & $\begin{array}{l}\text { Stärkung von Impulszentren durch (i) Erhöhung der Innen- } \\
\text { wirkung mit Nutzen für das Impulszentrum selbst und für die } \\
\text { darin angesiedelten Unternehmen, (ii) Erhöhung der Außen- } \\
\text { wirkung der Impulszentren durch Intensivierung der Zusam- } \\
\text { menarbeit bestehender Impulszentren untereinander als auch } \\
\text { mit geeigneten Bildungs-, Forschungs- und Infrastrukturein- } \\
\text { richtungen auf regionaler, nationaler und internationaler Basis } \\
\text { und insbesondere mit regionalen KMUs, (iii) Qualifizierungs- } \\
\text { maßnahmen für Impulszentren und deren Mitarbeiter und } \\
\text { Mitarbeiterinnen. } \\
\text { Verstärkter Einsatz moderner IKT } \\
\text { Stärkung von Regionen durch (i) Aufbau oder Erweiterung } \\
\text { von Kooperationsvorhaben und / oder Innovationsnetzwerken, } \\
\text { initiiert durch das Management von Impulszentren, (ii) Förde- } \\
\text { rung von Vorhaben und Projekten der Impulszentren (bzw. } \\
\text { der Impulszentren gemeinsam mit anderen Partnern), mit } \\
\text { denen systematisch der Wissensstand erhöht und für neue } \\
\text { Anwendungen in der Region genutzt wird, (iii) Bedarfsorien- } \\
\text { tierte Schließung von Lücken regionaler Innovationssyste- } \\
\text { me und Infrastrukturen durch Schaffung 'virtueller Impuls- } \\
\text { zentren' in Form dauerhafter, projektorientierter Trägerstruk- } \\
\text { turen für regionale Innovationsvorhaben }\end{array}$ & \begin{tabular}{|l|} 
Relevanz und \\
Angemessenheit \\
Qualitäts- und \\
Innovationssprung \\
Partnerstrukturen \\
Humanressourcen \\
Nutzen für den \\
Unternehmenssektor \\
Innovationsnetzwerke
\end{tabular} \\
\hline
\end{tabular}

Quelle: Technologie Impulse Gesellschaft, Sonderrichtlinien RIF 2000 - Regionale Impulsförderung, bmvit / TIG, Wien, 2002

\subsection{Der politische Kontext während der Entstehung des RIF 2000 Programms}

Seit Ende der 90er Jahre hat sich in der Forschungs-, Technologie- und Innovationspolitik in Österreich vieles verändert. Man ist bisweilen geneigt, eher von zwei verschiedenen Ländern zu sprechen als von einem, dass sich verändert hat. Tabelle 6 gibt einen knappen Überblick über die wichtigsten Veränderungen.

Tabelle 6 Veränderungen im institutionellen Umfeld seit Ende der 90er Jahre: Politik, Infrastrukturen, Finanzierung

\begin{tabular}{|l|l|}
\hline \multirow{4}{*}{ Politikorganisation } & Einrichtung des Rates für Forschung und Technologieentwicklung \\
\cline { 2 - 2 } & $\begin{array}{l}\text { Einrichtung der Nationalstiftung für Forschung, Technologie und } \\
\text { Entwicklung }\end{array}$ \\
\cline { 2 - 2 } & $\begin{array}{l}\text { Re-Organisation der Forschungsförderung durch die Einrichtung der } \\
\text { Forschungsförderungsgesellschaft (FFG) bzw. der Wirtschaftsförderung } \\
\text { (AWS) }\end{array}$ \\
\hline $\begin{array}{l}\text { Infrastrukturen in } \\
\text { Forschungs- und Aus- } \\
\text { und Weiterbildung }\end{array}$ & $\begin{array}{l}\text { Einrichtung von Kompetenzzentren und -netzwerke (Kplus, Kind/net) und } \\
\text { von Inkubatoren (AplusB) sowie die deutliche Aufstockung der Zahl der } \\
\text { Christian-Doppler-Labors }\end{array}$ \\
\cline { 2 - 2 } & $\begin{array}{l}\text { Ausbau des Fachhochschulsystems einschl. der Förderung seiner } \\
\text { Forschungsaufgabe (FHplus) }\end{array}$ \\
\hline
\end{tabular}




\begin{tabular}{|l|l|}
\hline & Re-Organisation des Universitätssystems (UG 2002) \\
\cline { 2 - 2 } & deutliche Veränderungen in der Politik der ÖAW und der LBG \\
\cline { 2 - 2 } & $\begin{array}{l}\text { Re-Organisation des Seibersdorf- und Arsenal-Konglomerats und Aufbau } \\
\text { der Austrian Research Centers }\end{array}$ \\
\cline { 2 - 3 } & Wachstumsinitiative bei den Kooperativen Forschungseinrichtungen (ACR) \\
\hline Finanzierung & Offensivprogramme des Bundes \\
\hline & $\begin{array}{l}\text { Übergang der Förderungspolitik der Ministerien durch weitgehende Still- } \\
\text { legung der Projektförderung zugunsten der Durchführung von Programmen }\end{array}$ \\
\hline & $\begin{array}{l}\text { Auftauchen der Bundesländer als maßgebliche Akteure der FTI-Politik } \\
\text { einschließlich des Erstarkens von Förder- und Entwicklungsagenturen }\end{array}$ \\
\hline & $\begin{array}{l}\text { generell: Steigerung der F\&E-Aufwendungen von 1,78\% [1998] auf 2,35\% } \\
\text { [2005] }\end{array}$ \\
\hline
\end{tabular}

In genau diese Phase fällt die Vorbereitung und Umsetzung des RIF 2000 Programms. Es ist daher interessant, den Entstehungszusammenhang von RIF $2000 \mathrm{im}$ Allgemeinen und einzelnen markanten Details im Besonderen zu rekonstruieren. Folgende Umstände und Überlegungen haben dabei auf ihre Weise eine Rolle gespielt.

- Im bmvit setzte sich die Vorstellung durch, dass im Bereich der Impulszentren ein Übergang zu 'weichen' Förderungen ansteht. Gefördert wurde diese Einsicht primär durch eigene Erfahrung, ergänzt, zugespitzt und explizit gemacht wurde durch sie die Evaluierung der Regionalen Innovationsförderung. Eine zweite Schlussfolgerung bestand darin, nicht mehr nur Zielgebiete, sondern Regionen generell zu fördern. Die Förderung von Regionen sollte nicht allein und nicht primär defizitgetrieben sein, sondern es sollte jede Region, nach Maßgabe ihrer Ausgangssituation und ihrer Möglichkeiten, gefördert werden können, also auch chancengetrieben. Dass es gerade die Impulszentren waren, kann als ein typischer Fall von Pfadabhängigkeit betrachtet werden, insofern hier Erfahrung und Beziehungen existierten, nicht zuletzt aus früheren Förderaktionen bzw. aus Eigentumsverhältnissen. Unter anderen Umständen ${ }^{6}$ hätte man vielleicht darauf abgezielt, die damals im Entstehen begriffenen regionalen Entwicklungsagenturen zum Partner von ko-finanzierten Programmen zu machen, etwa im Bereich der Clusterinitiativen ${ }^{7}$.

- Es gab Erfahrungen mit Regionen und vor allem von Impulszentren im Ressort und in der damals neu gegründeten Technologie Impulse Gesellschaft (TIG) bzw. ihrer Vorgängerorganisation. Diese Erfahrung resultiert vor allem aus der Beschäftigung mit Machbarkeitsstudien, die obligatorisch vor der Errichtung von Impulszentren durchgeführt wurden. Dazu kam die Erfahrung, dass es für die Manager der jeweiligen Zentren in der Regel schwierig war, "ihr Haus mit intelligenten Betrieben voll zu kriegen". Also mussten die Manager und ihr Umfeld überzeugt und gestärkt werden, ihr Geschäft in diese Richtung hin zu entwickeln.

- Es gab eine zweite, prägende Erfahrung, nunmehr in der TIG. Es war dies die erfolgreiche Vorbereitung und Einführung des Kplus Kompetenzzentrenprogramms, wo erstmals ein Programm von A bis Z durchgeplant worden ist. In der Retrospektive betrachtet, lässt sich das Kplus Programm als Paradigma für

$6 \quad$ Im Besonderen ist hier die Arbeitsteilung zwischen dem Vorgänger des heutigen Bundesministeriums für Verkehr, Innovation und Technologie bzw. dem heutigen Bundesministerium für Wirtschaft und Arbeit zu berücksichtigen.

7 Diese Partnerschaft ist ja inzwischen durch die Hintertür eingetreten: Ein nicht unerheblicher Teil der Bundesprogramme haben eine Ko-Finanzierung durch die Länder zur Bedingung. 
die nachfolgenden Programme, zumal innerhalb der TIG, identifizieren. Nicht umsonst haben die in den Folgejahren entwickelten Programme jeweils das Kürzel 'plus' enthalten ${ }^{8}$.

- Diese strenge, auf Explizitheit gerichtete Programmphilosophie wurde auch durch ein verändertes Regime im Bundesministerium für Finanzen sowie im damals neu gegründeten Rat für Forschung und Technologieentwicklung unterstützt. Beide hatten ihre Liebe zu Formalisierung und Quantifizierung entdeckt und damit - unvermeidbar - eine gewisse Schematisierung eingehandelt. Programme mussten durch Rückgriff auf harte Kriterien dargestellt und konnten so leichter 'verkauft' werden. Die Barcelona- und Lissabon-Ziele waren hoch im Kurs und Programme mussten dementsprechend zur Erreichung der entsprechenden Ziele beitragen können ${ }^{9}$.

- Die Tatsache, dass vor allem seit 1998 fleißig gebaut wurde (vgl. Abbildung 1), tut der Ausrichtung seitens des Bundes auf 'weiche' Maßnahmen keinen Abbruch, weil hier - nicht unschlau - die Leitvorstellung existierte, wonach die Zentren insbesondere die 'Zentren der Region' sein sollten. Bauen war daher oft auch als ein Signal zu verstehen, dass die Region in den physischen Zentren auch ein Zentrum der Aufmerksamkeit und damit der Unterstützung und Orientierung, nicht zuletzt des Zugangs zu 'ferneren' Unterstützungsangeboten (Land, Bund, EU) erhalten würden.

- Zusammenfassend kommen wir zu der Einschätzung, wonach die Entstehung des RIF 2000 Programms stark von internen Faktoren geprägt war. Diese sind (i) vorgängige Erfahrung, (ii) aufgebaute Strukturen im Ministerium und (iii) Erfahrung und Strukturen auf der Agenturseite. So wurden unter dem allgemeinen Titel 'Regionale Innovationsförderung' Impulszentren gefördert, nicht aber etwa Clusterinitiativen, ein damals viel versprechender Politikansatz auf Länderebene. Wegen dieser starken Innenorientierung gab es auch kaum Abstimmung mit verwandten Programmen. Im Vordergrund wäre hier vor allem das Bundesministerium für Wirtschaft und Arbeit gestanden, namentlich durch seine Zuständigkeit für das Programm Technologietransfer bzw. die 'Wachstumsförderung' der Kooperativen Forschungseinrichtungen oder die Patronanz für den Verband der Technologiezentren Österreichs (VTÖ). ${ }^{10}$ Alles in Allem betrachten wir das Zustandekommen des RIF 2000 Programms als typisches Ergebnis eine durch kontingente Faktoren frühzeitig determiniertes und durch Pfaddeterminiertheit stabilisiertes Programm.

\subsection{Auf welche Probleme war RIF 2000 die Antwort?}

Das RIF 2000 Programm zielt auf Impulszentren als Adressaten von Förderung ab. Es verfolgt aber nicht so sehr die Entwicklung von Impulszentren selbst, sondern eher die Entwicklung der regionalen Wirtschaft und versteht die jeweiligen Impulszentren

Es sind dies FHplus, REGplus, protec netplus und AplusB. Auch ein BplusC war angedacht. Dieses Problem der Schematisierung reicht bei Weitem nicht aus, um die Meriten dieser Vorgangsweise auch nur im Mindesten aufzuwiegen, vor allem, wenn man damals noch schwach entwickelte Programmkultur als Kontrast heranzieht.

10 Diese mangelnde Koordination wurde beim Nachfolgeprogramm des Technologietransferprogamms, protec, deutlich überwunden. Äußerer Ausdruck dieser Koordination ist die Aufspaltung von protec in zwei Programmlinien. 
als Entwicklungspol. Grundlage ist das Konzept des Innovationssystems. Das Programmdokument ist hier sehr explizit und eindeutig ${ }^{11}$.

\section{Förderungsmodul "REGplus"}

Mit diesem Förderungsmodul sollen Projekte gefördert werden, bei denen Impulszentren als Initiatoren, Träger oder Vermittler regionaler Innovationsprojekte auftreten. Im Vordergrund steht die Stärkung des Impulszentrums als Infrastruktureinrichtung und wesentlicher Träger von entsprechenden Initiativen. Zugleich soll die Innovationskraft der jeweiligen Region verbessert werden. Im Förderungsmodul "REGplus" wird zwischen "Aufbauprojekten" und "Erweiterungsprojekten" unterschieden.

\section{Aufbauprojekte}

Aufbauprojekte werden in der Regel eine beschränkte regionale Wirkung haben und können sich auch auf die Verbesserung der Struktur des Zentrums selbst beschränken. Sie werden in den meisten Fällen eine organisatorische Vorlaufphase für die Bildung von Kooperationen benötigen. Inhalte der Aufbauprojekte sind insbesondere:

- Maßnahmen und deren Umsetzungsstrategie zur Erhöhung der Attraktivität bestehender Impulszentren für vorhandene und potenzielle Mieter und zur Erhöhung des internen Dienstleistungsangebots;

- Planung und Umsetzung der inhaltlichen Positionierung im regionalen Umfeld, der Schwerpunktbildung und der regionalen Koordinationsfunktionen von bestehenden Impulszentren.

\section{Erweiterungsprojekte}

Erweiterungsprojekte sind anspruchsvolle Kooperationsprojekte mit modellhaftem Charakter unter Einbeziehung mehrerer Partner aus dem Infrastruktur- und Unternehmensbereich. Als Resultat wird die Erreichung eines insgesamt höheren Kooperations- und Innovationsniveaus der betroffenen Region erwartet. Inhalte der Erweiterungsprojekte sind insbesondere:

- Planung und Umsetzung von Vernetzungsaktivitäten sowie arbeitsteiliger, innovationsorientierter und regionaler Leistungspakete bestehender Impulszentren. Dies umfasst sowohl die Kooperation von Impulszentren untereinander als auch mit geeigneten Forschungs- und Bildungseinrichtungen sowie mit anderen Institutionen und mit Unternehmen.

\section{Förderungsmodul "Invest"}

Mit diesem Förderungsmodul sollen auf Basis projektbezogener Strategie- und Nutzungskonzepte Projekte gefördert werden, die eine verstärkte regionale Positionierung des Impulszentrums gewährleisten sowie Kapazitäts- und Ausstattungsprobleme lösen, insbesondere durch:

- Verbesserungen der technischen Ausstattung der Impulszentren;

- Ausbaustufen bestehender Impulszentren in begründeten Ausnahmefällen.

\section{Sonderprojekte}

Über die Förderungsmodule "REGplus" und "Invest" hinaus können weitere Upgrading-Projekte von Impulszentren gefördert werden, insbesondere nationale und internationale Vernetzungs- und Qualifikationsprogramme der Impulszentren. Dadurch soll das Qualifikationsniveau der Zentrumsmitarbeiter sowie die Integration der Zentren in Netzwerke von F\&E-Einrichtungen und anderer Impulszentren verbessert werden.

Dies ist ein Auszug aus der ersten Richtlinie aus dem Jahr 2000. In der überarbeiteten Form aus dem Jahr 2002 werden das Förderungsmodul 'Invest' und die Sonderprojekte durch 'RIFupgrade' ersetzt.

\section{Förderungsmodul "RIFupgrade"}

Mit diesem Förderungsmodul sollen auf Basis projektbezogener Strategie- und Nutzungskonzepte Projekte gefördert werden, die eine verstärkte regionale Positionierung des Impulszentrums gewährleisten, Kapazitäts- und Ausstattungsprobleme lösen und/oder das Qualifikationsniveau der Zentrumsmitarbeiter verbessern oder sonst zu einem qualitativem Upgrading des Impulszentrums beitragen, d.h. Projekte für

- technologisches und qualitatives Upgrading der Impulszentren;

11 Bundesministerium für Verkehr, Innovation und Technologie, Sonderrichtlinien für die RIF 2000 - Regionale Impulsförderung. Laufzeit 2000 - 2006. Entwurf vom 20.11.2000 
Das RIF 2000 Programm zielt auf Regionalentwicklung durch Innovation bzw. Kooperation ab. Unmittelbarer Adressat sind Impulszentren einschließlich so genannter 'virtueller Impulszentren'. Dabei dominieren zwei, aufeinander bezogene Interventionsmodelle: (i) Stärkung des Impulszentrums als Infrastruktureinrichtung und wesentlicher Träger von entsprechenden Initiativen, (ii) Kooperation und Vernetzung von Impulszentren untereinander als auch mit geeigneten Forschungsund Bildungseinrichtungen sowie mit anderen Institutionen und mit Unternehmen. Offenkundig dient das erste Interventionsmodell als Voraussetzung zur effizienten Nutzung des zweiten.

Man könnte einwenden, dass zur Erreichung der gesetzten Ziele bzw. der Ausübung des zweiten Interventionsmodells auch andere Zielgruppen bzw. Akteure zur Verfügung gestanden wären: regionale Entwicklungsagenturen, Clusterinitiativen, Forschungseinrichtungen, Bildungseinrichtungen, Interessenvertretungen, Berater ${ }^{12}$. Grundlage dieses Einwands ist die Tatsache, dass diese unterschiedlichen Institutionen eine bisweilen größere Reichweite und größere Flexibilität aufweisen und sie nicht an die Existenz eines Impulszentrums angewiesen sind. Warum beschränkt man sich also, wenn es um Regionalentwicklung durch Innovation und Kooperation geht, auf diese doch sehr spezifische Zielgruppe der Impulszentren?

Wir teilen diesen Einwand nur sehr eingeschränkt, u.zw. wesentlich durch eine historische Betrachtung. Wir befinden uns, als über das RIF 2000 Programm nachgedacht wird, in den Jahren 1999-2000. Hier war das meiste, was wir heute kennen, noch gar nicht existent oder bestenfalls im status nascendi. Auf Bundesebene lief noch das Technologietransfer- und das FINT-Programm. Clusterinitiativen waren noch rar, neben dem Steirischen Automobilcluster gab es in Oberösterreich den Automobil- und den Kunststoffcluster, Salzburg richtete gerade den Cluster 'Digitale Medien' und das Innovationsservice ein. Die TMG war mit sich selbst, d.h. mit seinem großen 'Strategischen Programm OÖ 2000+' beschäftigt, das ZIT existierte noch gar nicht, die Tiroler Zukunftsstiftung war in Umbau begriffen, desgleichen das Techno-Z Salzburg, Niederösterreich war mit der Technologieoffensive und der Gründung der TecNetCompany beschäftigt. Insgesamt gab es entweder noch keine ausgeprägte Struktur - weder auf Landes- noch auf Bundesebene -, oder es waren die diversen Institutionen ihrerseits vorwiegend mit sich selbst und mit den Planungen über die Verwendung der Ende der 90er Jahre massiv angewachsenen Landesgelder beschäftigt. Dazu kommt die erwähnte starke Innensteuerung des RIF 2000 Programms auf Seiten der handelnden Personen im Ressort und in der TIG bzw. ihrer Vorgängerorganisation.

Wenn man heute, 2005, einwendet, dass im RIF 2000 Programm technologie- und innovationsorientierte Regionalentwicklung gefördert wurde bzw. wird und man sich dabei auf ein einziges Instrument, nämlich die Impulszentren, festgelegt und dabei zahlreiche andere Instrumente nicht benutzt hat, so tut man dies aus einer geschichtslosen Betrachtung heraus. Eine genauere Betrachtung zeigt vielmehr, dass die heute in Hülle und Fülle vorhandenen Förderangebote damals, also 1999-2000, zu großen

12 Man beachte: Es handelt sich um dieselben Arten von Organisationen bzw. Institutionen, die auch Zielgruppe etwa von CIR-CE oder protec sind. 
Teilen weder zur Verfügung gestanden sind, noch absehbar war, wie sich das Politiksystem sowohl auf Bundesebene wie auch auf Ebene der Länder entwickeln würde ${ }^{13}$.

Die Tatsache, dass in den Jahren 1997 ff. ein Großteil der heute existierenden Impulszentren erst gegründet wurde (vgl. Abbildung 1), kann im Nachhinein eher als eine Bestätigung der im Rahmen des RIF 2000 Programms gewählten Strategie, nämlich Regionalentwicklung über Impulszentren zu betreiben, angesehen werden. Für die Programmverantwortlichen war die Vermehrung der Impulszentren eine Verbesserung der Situation, insofern zu erwarten war, dass eine größere Zahl an Zentren die Wahrscheinlichkeit erhöhen würde, genügend gute Projekte zu finden. Wäre es das Ziel von RIF 2000 gewesen, die Versorgungsdichte mit Impulszentren, verstanden hier als Inkubator für junge Unternehmen, zu erhöhen, so wäre dies in der Tat problematisch gewesen, insofern die Schaffung von hochwertigen Immobilien tatsächlich kein gravierender Engpass war und ist. Was hingegen die Versorgung der regionalen, besser noch: der lokalen Wirtschaft mit einschlägigen Dienstleistungen und Unterstützungen betrifft, so wurde durch das RIF 2000 Programm Neuland betreten und ein tatsächlich vorhandenes Problem angesprochen. Das Problem, um das es sich handelt, ist die Versorgung der lokalen Wirtschaft - definiert hier als die in einem Einzugsbereich von einer halben bis max. einer Stunde Fahrzeit mit dem Auto - mit unterschiedlichen innovationsunterstützenden Dienstleistungen.

\section{Schlussfolgerungen}

Eine Analyse der Zielsetzungen und darin eingebauten Strategien von RIF 2000 lässt natürlich Zweifel über die Angemessenheit der gewählten Strategie aufkommen, nämlich mit Hilfe von Impulszentren technologie- und innovationsorientierte Regionalentwicklung zu betreiben. Unser Zugang indes, der die Entstehungsgeschichte und den damaligen Kontext in den Mittelpunkt stellt, führt zu einem deutlich anderen Schluss. Demnach gab es gute Gründe, das RIF 2000 Programm aufzulegen und es in der vorliegenden Form auszurichten. Dass man allerdings, zweifellos in Kenntnis des bevorstehenden Booms bei den Zentren ab 1998, noch einen Förderungsmodel 'Invest' vorgesehen hatte, zeugt von einem gewissen Nachwirken das alten Förderansatzes ${ }^{14}$. In einer kontextlosen Betrachtung wäre dieses Modul zweifellos als entbehrlich beurteilt worden. Im Lichte der zuvor und über viele Jahre geübten Praxis und vor dem Hintergrund, dass der Wechsel zu einem weitgehend neuen Förderungsregime durchaus mit Unsicherheit und Risiko behaftet war, lässt sich verstehen, dass man die alte Förderlogik nicht gänzlich aufgeben wollte.

13 Wie unsicher und wie schwer vorhersehbar die Zukunft in dem hier betrachteten Politiksegment ist, kann man daran erkennen, dass, obwohl in den nächsten zwei Jahren die meisten Kompetenzzentren auslaufen, sich nach nunmehr anderthalb Jahren Beratung noch immer keine stabile Lösung abzeichnet.

14 Dieses Förderungsmodul 'Invest' wurde schließlich ohnehin nicht umgesetzt und fand in RIFupgrade eine modernere Entsprechung. 


\section{Die Durchführung des RIF 2000 Programms}

Die beiden Förderungsmodule 'RIFupgrade' und 'REGplus' unterscheiden sich in ihrer Handhabung erheblich. Wir wollen dies im Folgenden analysieren und stellen die jeweiligen Elemente in einer Matrix gegenüber.

\subsection{Vergleich der Abwicklungsprozeduren der beiden Förderungsmodule}

Zwischen RIFupgrade und REGplus liegen Welten und Kulturen. Während RIFupgrade noch dem traditionellen, über Jahrzehnte gepflogenen Modell der Antragsförderung in Verbindung mit Begutachtung durch den Förderer verpflichtet ist, folgt die Projektakquisition bei REGplus über das modernere Konzept der Ausschreibung bzw. Wettbewerben und die Begutachtung der Projekte durch Fachexperten bzw. eine Jury. Die Wahl des Ausschreibungs- und Juryprinzips war historisch zweifellos inspiriert von der einige Jahre zuvor eingeführten Praxis im Rahmen des Kplus Kompetenzzentrenprogramms, das als Paradigma der Technologie Impulse Gesellschaft für die Art der Projektakquisition und Begutachtung gelten kann. Tabelle 7 stellt die Details der Abwicklungsprozeduren der beiden Förderungsmodule im Vergleich dar.

Tabelle 7 Die Abwicklungsprozeduren der beiden Förderungsmodule im Vergleich

\begin{tabular}{|c|c|c|}
\hline RIFupgrade & REGplus & Kommentar \\
\hline \multicolumn{3}{|l|}{ Abwicklung des Fördermoduls } \\
\hline $\begin{array}{l}\text { Mit der Abwicklung dieses Förderungs- } \\
\text { moduls ist die Abteilung V/2 des bmvit als } \\
\text { Förderungsabwicklungsstelle betraut. }\end{array}$ & $\begin{array}{l}\text { Mit der Abwicklung dieses Förderungs- } \\
\text { moduls ist die TIG als Förderungsabwick- } \\
\text { lungsstelle betraut. Das Förderungsmodul } \\
\text { 'REGplus' wird in Form von Wettbewerben } \\
\text { durchgeführt. Die TIG führt in Abstim- } \\
\text { mung mit dem bmvit während der Laufzeit } \\
\text { dieser Sonderrichtlinien Ermittlungsver- } \\
\text { fahren zur Förderung von Projekten in } \\
\text { Form offener Ausschreibungen durch. Die } \\
\text { detaillierte Verfahrensabwicklung wird in } \\
\text { der Ausschreibung geregelt. }\end{array}$ & $\begin{array}{l}\text { RIFupgrade Projekte } \\
\text { können jederzeit, } \\
\text { REGplus nur im Zuge } \\
\text { von Ausschreibungen } \\
\text { eingeworben werden. }\end{array}$ \\
\hline \multicolumn{3}{|l|}{ Einreichung des Förderungsansuchens } \\
\hline $\begin{array}{l}\text { Förderungsansuchen können - vorbehalt- } \\
\text { lich einer vorzeitigen Revision - unter } \\
\text { Verwendung des dafür vorgesehenen For- } \\
\text { mulars mit den erforderlichen Unterlagen in } \\
\text { zweifacher Ausfertigung bis zum 31.7.2006 } \\
\text { beim Bundesministerium für Verkehr, } \\
\text { Innovation und Technologie (bmvit), } \\
\text { Abt.V/2, Renngasse 5, } 1010 \text { Wien } \\
\text { eingereicht werden. }\end{array}$ & $\begin{array}{l}\text { Die Förderungsansuchen sind gemäß dem } \\
\text { 'Leitfaden für Antragsteller' bei der TIG, } \\
\text { Grillparzerstraße 7/Top 8, } 1010 \text { Wien } \\
\text { einzureichen. }\end{array}$ & \\
\hline
\end{tabular}




\begin{tabular}{|c|c|c|}
\hline RIFupgrade & REGplus & Kommentar \\
\hline \multicolumn{3}{|l|}{ Prüfung des Förderungsansuchens } \\
\hline $\begin{array}{l}\text { Die formgerecht eingebrachten Förderungs- } \\
\text { ansuchen sind von der Abt. V/2 des bmvit } \\
\text { im Sinne der Richtlinien zu prüfen. Im Falle } \\
\text { einer möglichen EU-Kofinanzierung ist } \\
\text { frühzeitig (bei verpflichtender EU-Kofinan- } \\
\text { zierung mit der Antragstellung) das Vor- } \\
\text { liegen der Absichtserklärung der zuständig- } \\
\text { en maßnahmenverantwortlichen Förde- } \\
\text { rungsstelle zu prüfen. Bei unvollständigen } \\
\text { Förderungsansuchen ist durch die Abt. V/2 } \\
\text { des bmvit eine Verbesserung binnen ange- } \\
\text { messener Frist zu verlangen. Kommt diese } \\
\text { nicht zustande, so ist das unvollständige } \\
\text { Förderungsansuchen außer Evidenz zu } \\
\text { nehmen und dem Förderungswerber rück- } \\
\text { zumitteln. Ansuchen, die nicht den Richt- } \\
\text { linien entsprechen, sind auszuscheiden und } \\
\text { dem Förderungswerber mit Begründung } \\
\text { rückzumitteln. Die Begutachtung des } \\
\text { Förderungsansuchens erfolgt durch die Abt. } \\
\text { V/2 des bmvit. Im Bedarfsfall werden } \\
\text { ergänzende Expertengutachten (betriebs- } \\
\text { wirtschaftlich, regionalwirtschaftlich, } \\
\text { fachspezifisch) eingeholt. }\end{array}$ & $\begin{array}{l}\text { Die formale Prüfung auf Vollständigkeit } \\
\text { des Förderungsansuchens gemäß Leitfaden } \\
\text { ('eligibility check') erfolgt durch die TIG } \\
\text { und entscheidet über die weitere Behand- } \\
\text { lung des Förderungsansuchens. Die } \\
\text { Prüfung und Beurteilung der Qualität der } \\
\text { verpflichtenden Kriterien erfolgt durch die } \\
\text { Mitglieder einer einzurichtenden unabhän- } \\
\text { gigen ExpertInnenjury. Dabei muss jedes } \\
\text { verpflichtende Kriterium adäquat } \\
\text { vorhanden und erfüllt sein. Die inhaltliche } \\
\text { ('technisch-fachliche') Prüfung der qualita- } \\
\text { tiven Kriterien erfolgt ebenfalls durch die } \\
\text { ExpertInnenjury. Dabei ist zu beachten, } \\
\text { dass in den Förderungsansuchen nicht } \\
\text { jedes qualitative Kriterium dargestellt sein } \\
\text { muss. Die ExpertInnenjury wird durch den } \\
\text { Bundesminister für Verkehr, Innovation } \\
\text { und Technologie eingesetzt. Diese Exper- } \\
\text { tInnenjury, für die eine Geschäftsordnung } \\
\text { erlassen wird, führt eine Bewertung und } \\
\text { Reihung der Projekte durch und gibt auf } \\
\text { dieser Basis eine Förderungsempfehlung } \\
\text { ab. }\end{array}$ & $\begin{array}{l}\text { Bei RIFupgrade prüft } \\
\text { die Förderstelle und } \\
\text { der ERP-Fonds das } \\
\text { Ansuchen, bei } \\
\text { REGplus wird die } \\
\text { Formalprüfung von der } \\
\text { Abwicklungsstelle } \\
\text { durchgeführt, die } \\
\text { technisch-fachliche } \\
\text { Prüfung von einer } \\
\text { Jury. } \\
\text { Bei RIFupgrade } \\
\text { besteht die } \\
\text { Möglichkeit einer } \\
\text { Nachbesserung des } \\
\text { Ansuchens, bei } \\
\text { REGplus nicht. }\end{array}$ \\
\hline \multicolumn{3}{|l|}{$\begin{array}{l}\text { Entscheidung über das } \\
\text { Förderungsansuchen }\end{array}$} \\
\hline $\begin{array}{l}\text { Die Förderungsansuchen werden von der } \\
\text { Abt. V/2 des bmvit mit einer Zusammen- } \\
\text { fassung aller Gutachten und einer Förde- } \\
\text { rungsempfehlung dem Bundesminister für } \\
\text { Verkehr, Innovation und Technologie } \\
\text { möglichst innerhalb von } 3 \text { Monaten nach } \\
\text { Vorliegen des vollständigen Förderungsan- } \\
\text { suchens vorgelegt. Die } \\
\text { Förderungsentscheidung trifft der } \\
\text { Bundesminister für Verkehr, Innovation } \\
\text { und Technologie nach Prüfung der } \\
\text { Förderungsempfehlung. Die } \\
\text { Förderungsentscheidung ist dem } \\
\text { Förderungswerber im Falle einer Ableh- } \\
\text { nung unter Angabe von Gründen, sonst in } \\
\text { Form eines Förderungsanbots schriftlich } \\
\text { mitzuteilen. }\end{array}$ & $\begin{array}{l}\text { Die Förderungsentscheidung trifft der } \\
\text { Bundesminister für Verkehr, Innovation } \\
\text { und Technologie nach Prüfung der } \\
\text { Förderungsempfehlung. Die Förderungs- } \\
\text { entscheidung ist dem Förderungswerber im } \\
\text { Falle einer Ablehnung unter Angabe von } \\
\text { Gründen, sonst in Form eines } \\
\text { Förderungsanbots schriftlich mitzuteilen. }\end{array}$ & $\begin{array}{l}\text { In beiden Modulen } \\
\text { entscheidet der } \\
\text { Bundesminister. } \\
\text { Ablehnungen müssen } \\
\text { in beiden Fällen } \\
\text { begründet werden. }\end{array}$ \\
\hline \multicolumn{3}{|l|}{ Förderungsanbot / Förderungsvertrag } \\
\hline $\begin{array}{l}\text { Das Förderungsanbot enthält die detaillier- } \\
\text { ten Förderungsbedingungen/- auflagen und } \\
\text { bedarf der schriftlichen Annahme. Es gilt } \\
\text { als zurückgezogen, wenn die Annahme } \\
\text { nicht binnen } 2 \text { Monaten ab Zustellung bei } \\
\text { der Abt. V/2 des bmvit einlangt. Mit der } \\
\text { Annahme kommt der Förderungsvertrag } \\
\text { zustande. }\end{array}$ & $\begin{array}{l}\text { Das Förderungsanbot enthält die } \\
\text { detaillierten Förderungsbedingungen/- } \\
\text { auflagen. Auf Basis des Antrags, der } \\
\text { Förderungsentscheidung, allfälliger } \\
\text { Förderungsauflagen der ExpertInnenjury } \\
\text { und etwaiger Erläuterungen und Spezi- } \\
\text { fikationen erstellt die TIG den Förderungs- } \\
\text { vertrag, der sowohl vom Förderungsgeber } \\
\text { als auch vom Förderungsnehmer zu unter- } \\
\text { zeichnen ist. In diesem Vertrag sind auch } \\
\text { die Einstellungs- und Rückforderungs- } \\
\text { bedingungen anzuführen. }\end{array}$ & \\
\hline
\end{tabular}




\begin{tabular}{|c|c|c|}
\hline RIFupgrade & REGplus & Kommentar \\
\hline Auszahlungen & & \\
\hline $\begin{array}{l}\text { Die Auszahlung der Förderung erfolgt nach } \\
\text { Projektfortschritt sowie Prüfung und } \\
\text { Annahme der vom Förderungsnehmer zu } \\
\text { vereinbarten Meilensteinen fristgerecht } \\
\text { vorzulegenden Unterlagen und Nachweise. } \\
\text { Bei nachgewiesenem Bedarf kann eine } \\
\text { Akontierung der Förderung erfolgen. In } \\
\text { diesem Fall ist der Förderungsnehmer zu } \\
\text { verpflichten, Zinsen einer kurzfristigen } \\
\text { Veranlagung für den Widmungszweck zu } \\
\text { verwenden. Die Prüfung der gemäß Abs.(1) } \\
\text { vorzulegenden Belege und sonstigen } \\
\text { Nachweise erfolgt in Verantwortung des } \\
\text { Kontrollreferates der Sektion V des bmvit. } \\
\text { Das Prüfungsergebnis ist der Abt. V/2 des } \\
\text { bmvit mitzuteilen. Werden die } \\
\text { vorgesehenen förderbaren Projektkosten } \\
\text { unterschritten und sind die } \\
\text { Förderungsvoraussetzungen weiterhin } \\
\text { gegeben, verringert sich die Förderung } \\
\text { aliquot. Dies ist dem Förderungsnehmer } \\
\text { durch die Abt. V/2 des bmvit schriftlich } \\
\text { mitzuteilen. }\end{array}$ & $\begin{array}{l}\text { Die Auszahlungen erfolgen im Rahmen } \\
\text { des genehmigten Budgetplans mit einer } \\
\text { Startrate am Beginn des Projektes nach } \\
\text { Vertragsunterzeichnung und in der Folge } \\
\text { des Projektes nach Bedarf und auf } \\
\text { Grundlage der jeweils im Jahresbericht } \\
\text { (Pkt. 7.3.) vorgelegten Budgetansätze } \\
\text { (Planziffern) für das Folgejahr. Sie werden } \\
\text { an eine Meilensteinkontrolle gekoppelt. } \\
\text { Die letzte Auszahlung ist an die } \\
\text { Genehmigung des Endberichtes gebunden. } \\
\text { Werden die vorgesehenen förderbaren } \\
\text { Projektkosten unterschritten und sind die } \\
\text { Förderungsvoraussetzungen weiterhin } \\
\text { gegeben, verringert sich die Förderung } \\
\text { aliquot; gegebenenfalls sind bereits } \\
\text { ausbezahlte Förderungsmittel verzinst } \\
\text { zurückzuzahlen. Dies ist dem } \\
\text { Förderungsnehmer durch die TIG } \\
\text { schriftlich mitzuteilen. Der } \\
\text { Förderungsnehmer ist zu verpflichten, } \\
\text { Zinsen einer kurzfristigen Veranlagung für } \\
\text { den Widmungszweck zu verwenden. }\end{array}$ & $\begin{array}{l}\text { Bei beiden Förde- } \\
\text { rungsmodulen besteht } \\
\text { die Möglichkeit einer } \\
\text { Akontierung der } \\
\text { Förderung. Ansonsten } \\
\text { erfolgt in beiden } \\
\text { Fällen die Auszahlung } \\
\text { auf Basis von } \\
\text { Meilensteinen bzw. bei } \\
\text { REGplus auf Basis der } \\
\text { Jahresberichte und des } \\
\text { Schlussberichts } \\
\text { (10\%ige Schlussrate } \\
\text { der Förderung nach } \\
\text { Projektende). }\end{array}$ \\
\hline
\end{tabular}

Quelle: Bundesministerium für Verkehr, Innovation und Technologie, Sonderrichtlinien für die RIF

2000 - Regionale Impulsförderung. Laufzeit 2000 - 2006. Stand 2002

\subsection{Wahrnehmung und Handhabung des Ausschreibungsprinzips}

Wir wollen im Folgenden näher auf die Wahrnehmung und Handhabung dieses neuen Prinzips eingehen. Generell kann man behaupten, dass dieser Modus von allen an diesem Prozess Beteiligten positiv aufgenommen wurde und im Vergleich mit dem Antragsprinzip eindeutig favorisiert wird.

Tabelle 8 Ablauf des Ausschreibungsverfahrens im Fördermodul REGplus

\begin{tabular}{|c|c|c|c|c|}
\hline Aufgabe / Arbeitsschritt & $\begin{array}{l}\text { TIG / } \\
\text { FFG }\end{array}$ & Gutachter & Jury & $\begin{array}{l}\text { Bundes- } \\
\text { minister }\end{array}$ \\
\hline Veröffentlichung der Ausschreibung, Laufzeit 3-4 Monate & $\mathrm{x}$ & & & \\
\hline $\begin{array}{l}\text { Durchführung von Informationsveranstaltungen ('Proposers } \\
\text { Days') }\end{array}$ & $\mathrm{X}$ & & & \\
\hline Formalprüfung ('elegibility check') & $\mathrm{x}$ & & & \\
\hline Versendung der eingereichten Anträge an die Gutachter & $\mathrm{x}$ & & & \\
\hline $\begin{array}{l}\text { schriftliche Begutachtung der Anträge durch die einzelnen } \\
\text { Gutachter }\end{array}$ & & $\mathrm{x}$ & & \\
\hline Kompilation der eingegangenen Gutachten und Rangreihung & $\mathrm{x}$ & & & \\
\hline $\begin{array}{l}\text { Sitzung der Jury, Mitarbeiter der TIG / FFG nehmen als } \\
\text { Auskunftspersonen teil, } 1 \text { Vertreter des bmvit mit Vorsitz- } \\
\text { funktion }\end{array}$ & & & $\mathrm{X}$ & \\
\hline Entscheidung & & & & $\mathrm{X}$ \\
\hline Verhandlungen (auf Basis von Auflagen) & $\mathrm{x}$ & & & \\
\hline
\end{tabular}

Quelle: TIG / FFG

Der in Tabelle 8 beschriebene Prozess dauert ca. 6 Monate. Davon verstreichen 3-4 Monate für die Ausschreibungsfrist. Von den Förderungswerbern wird diese Frist unisono als optimal empfunden. Sie ist lang genug, um Projektideen zu entwickeln, Partner zu suchen, diese auf das Projekt einzustimmen und den Antrag zu verfassen. Letzteres geschieht dann in den letzten 3-4 Wochen vor Einreichschluss. Tatsächlich 
zeigt sich, dass die meisten Zentrumsmanager ${ }^{15}$ immer einige Projektideen 'in der Pipeline' haben und die Ausschreibung dementsprechend eine Möglichkeit darstellt, diese aufzugreifen ${ }^{16}$. Und sie ist kurz genug, um die Aufmerksamkeit nicht abreißen zu lassen und die entsprechenden Ressourcen zu disponieren ${ }^{17}$.

Das Ausschreibungsprinzip im Sinne eines fixen Einreichtermins wird durchwegs auch unter dem Gesichtspunkt der Verbesserung der Qualität gesehen ${ }^{18}$. Wir finden neben diesem auf höhere Qualität gerichteten Additionalitätseffekt auch noch einen weiteren Zusatzeffekt des Ausschreibungsprinzips vor. Es wird zwar durchwegs die Ansicht vertreten, dass ein Zentrumsmanager immer mit mehreren Projekten schwanger geht. Vergleichsweise selten werden diese aber in der Folge auch aufgegriffen und umgesetzt. Das Ausschreibungsprinzip wird daher von den meisten Managern daher auch deshalb begrüßt, weil es als Auslöser dafür dient, dass unausgegorene Ideen, hängende Partnerschaften ('weak ties') und das eine oder andere Problem tatsächlich aufgegriffen und konkretisiert und im Falle des Gewinnens auch in Angriff genommen werden. Das Ausschreibungsprinzip dient also als eine Gewähr für qualitätsvollere Projekte.

Natürlich gibt es auch Stimmen, die das eine haben und das andere nicht missen wollen. Wir können dieser Idee nicht folgen und führen folgende Gründe ins Treffen. Es fällt schwer, eine Grenze zwischen unterschiedlichen Projekttypen festzulegen, wonach die einen nach dem Antragsprinzip und die anderen nach dem Ausschreibungsprinzip behandelt werden. Dies umso mehr, als die Projekte, die in den drei Ausschreibungsrunden eingereicht bzw. gefördert wurden, eine Bandbreite hinsichtlich Inhalt und Qualität aufweisen, die, wenn man sie einzelnen Gutachtern überließe, zu weithin erratischen Ergebnissen führen würde ${ }^{19}$.

Ein legitimer Wunsch und auch Vorschlag für die künftige Praxis von REGplusartigen Programmen besteht darin, dass es (i) eine Art Vorschau bei Ausschreibungen geben sollte und, darin impliziert, (ii) eine Abstimmung mit anderen einschlägigen Ausschreibungen.

Der Einsatz von Jurys als Begutachtungsinstrument wird ausnahmslos begrüßt. Sie wird als Beitrag zur Objektivierung empfunden und vermeidet dadurch das Überhandnehmen von ad hoc Entscheidungen ${ }^{20}$. Darüber hinaus wird der höhere Qualitätsan-

15 Mit 'Zentrumsmanager' meinen wir hier nicht allein die Geschäftsführer von Impulszentren i.e.S., sondern jene Gruppe von Personen, die in einer nennenswerten Weise zum Fortkommen des Zentrums beitragen, also Personen mit Führungs- und Gestaltungsaufgaben.

16 Dies impliziert, dass diese Aufmerksamkeit generell gilt und auch nach anderen Möglichkeiten der Förderung bzw. Finanzierung gegriffen wird. Wir werden weiter unten (Tabelle 23) auf dieses spezifische Suchverhalten noch einmal eingehen und es auch überwiegend bestätigt finden.

17 Dass es dann manchmal zu schnell geht, zeigt sich daran, dass sich vielfach der Start des Projekts verzögert.

18 Paradoxerweise, denn die Alltagswahrnehmung lautet ganz anders: Hätten wir noch ein paar Tage mehr Zeit, würden wir alles noch einmal gründlich durcharbeiten.

19 Wir werden weiter unten (Tabelle 16) zeigen, dass mehr als die Hälfte der Projekte bei den Vorab-Bewertungen durch die Jurymitglieder Noten von 0 (Minimum) bis 4 (Maximum) erhalten haben.

20 Wir unterstellen hier, dass die ablehnende Haltung gegenüber ad hoc Entscheidungen überwiegend eine klimatische Wahrnehmung ist und weniger auf spezifische Projekte Bezug nimmt, umso mehr als die Abwicklung der RIFupgrade Projekte aus der Sicht der Förderempfänger klaglos funktioniert hat. 
spruch gewürdigt und eine Bewertung durch die Jury als Benchmark und Referenz empfunden. Allerdings gibt es fallweise den Vorschlag bzw. die Forderung nach einem Hearing, wo die Projektwerber ihr Projekt vertreten können. Geht man von 2530 Anträgen aus und würde man jedem Projektwerber 15-20 Minuten einräumen, so würde sich die Sitzungsdauer der Jury von zwei auf drei Tage verlängern. Wir stehen diesem Vorschlag durchaus distanziert gegenüber, weil der Zusatznutzen ${ }^{21}$ aus diesem Unterfangen sich in Grenzen hält. Einen kleinen Hinweis, der diese Position untermauert, haben wir dadurch gewinnen können, als es eine große Übereinstimmung bei jenen Förderungswerbern gibt, die sowohl Projekte gewonnen als auch verloren haben: Ihre 'innere Stimme' ist über weite Strecken identisch mit jener der Jury.

Informationspolitik und Unterstützung während der Einreichphase. Die TIG / FFG hat während der Ausschreibungsfrist regelmäßig Informationsveranstaltungen durchgeführt. Diese werden als nützlich empfunden und es gibt kaum einen Förderwerber, der nicht eine dieser Veranstaltungen besucht hätte. Darüber hinaus werden persönliche Gespräche bzw. Telefonate mit der Förderabwicklungsstelle als hilfreich, gehaltvoll und objektiv empfunden. Es gibt Hinweise darauf, dass diese beiden Quellen Informationsveranstaltung vs. persönliches Gespräch / Telefonat - nicht zueinander substituierbar sind. Die Informationsveranstaltung scheint vielmehr eine gute Grundlage dafür zu sein, dass man im Rahmen eines persönlichen Gesprächs / Telefonats spezifische Fragen besprechen kann $^{22}$.

21 Diesem marginalen Nutzen stehen marginale (Opportunitäts)Kosten von bis zu EUR 100.000 gegenüber: Honorare für die Jurymitglieder, (Opportunitäts)Kosten bei den Vertretern der Förderabwicklungsstelle, vor allem aber (Opportunitäts)Kosten bei den Förderungswerbern, die wenn sie zu Zweit oder Dritt kommen, dramatisch ansteigen.

22 Die Informationsveranstaltung hat offenbar den Status eines Hygienefaktors - Hygiene nützt zwar nichts, wenn man sie aber unterlässt, entsteht Schaden. 


\section{Das REGplus Programm in Zahlen}

In diesem Kapitel steht das REGplus Programm im Vordergrund. In Kapitel 4.1 wird eine Übersicht über die geförderten Projekte, über beantragte und ausgeschüttete Förderungen gegeben. Dabei zeigt sich, dass es zu Kürzungen sowohl bei den anerkannten Projektkosten als auch bei den Förderintensitäten kam und darüber hinaus in großem Umfang Auflagen ausgesprochen wurden. Diesem Fragenkomplex gehen wir in Kapitel 4.2 nach. In Kapitel 4.3 diskutieren wir die Trefferbilanz nach Bundesländern. Dies ist insofern von Bedeutung, als die Bundesländer immerhin als Ko-Finanzier auftreten und man die begründete Vermutung hegen kann, dass hier unterschiedliche Strategien zum Tragen kommen, was sich denn auch bei näherer Betrachtung als zutreffend erweist.

\subsection{Geförderte Projekte, beantragte Förderungen, ausgeschüttete Förderungen}

In diesem Kapitel geben wir einen Überblick über die Eckdaten des Förderungsmoduls REGplus. In Tabelle 9 sind die eingereichten, geförderten und abgelehnten Projekte dargestellt. Darin zeigt sich zunächst, dass etwas weniger als die Hälfte $(47,4 \%)$ der eingereichten Projekte gefördert wurden. Vergleicht man dies mit den Erfolgsquoten aus vergleichbaren Programmen (Tabelle 10), so liegt REGplus etwa im Mittelfeld. Vergleicht man sie mit den im FFF üblichen Erfolgsquoten ${ }^{23}$, so liegen alle neueren Programmlinien signifikant darunter. Interessant ist, dass diese Ablehnungsquoten, abgesehen von einigen Ausreißern, von den jeweiligen Zielgruppen durchaus akzeptiert werden.

Tabelle 9 Eingereichte, geförderte und abgelehnte Projekte im Förderungsmodul REGplus

\begin{tabular}{|l|c|c|c|c|c|c|c|c|c|c|}
\hline & \multicolumn{3}{|c|}{ eingereicht } & \multicolumn{3}{c|}{ gefördert } & \multicolumn{3}{c|}{ nicht gefördert } & Anteil geförderte Projekte \\
\hline & AP & EP & gesamt & AP & EP & gesamt & AP & EP & gesamt & \\
\hline 1. Runde & 17 & 6 & 23 & 5 & 4 & 9 & 12 & 2 & 14 & $39 \%$ \\
\hline 2. Runde & 14 & 14 & 28 & 7 & 8 & 15 & 7 & 6 & 13 & $54 \%$ \\
\hline 3. Runde & 12 & 13 & 25 & 4 & 8 & 12 & 8 & 5 & 13 & $48 \%$ \\
\hline Gesamt & 43 & 33 & 76 & 16 & 20 & 36 & 27 & 13 & 40 & $47 \%$ \\
\hline
\end{tabular}

Quelle: FFG

23 Wie ersichtlich, schwanken auch diese durchaus in beachtlichem Ausmaß. Die angegebenen Prozentsätze sind die Maxima und Minima im Zeitraum 1995-2004. 
Tabelle 10 Ablehnungsquote bei ausgewählten Programmlinien

\begin{tabular}{|l|l|c|}
\hline \multicolumn{2}{|c|}{ Förderlinie } & Anteil geförderte Projekte [\%] \\
\hline FFF & neue Projekte (1997) & 75 \\
\hline & neue Projekte (2002) & 62 \\
\hline FHplus & 1. Runde & 30 \\
\hline & 2. Runde & 33 \\
\hline protec netplus & 1. Runde & 29 \\
\hline & 2. Runde & 35 \\
\hline & 3. Runde & 52 \\
\hline protec inno & 1. Runde & 32 \\
\hline & 2. Runde & 40 \\
\hline & 3. Runde & 40 \\
\hline REGplus & 1. Runde & 39 \\
\hline & 2. Runde & 48 \\
\hline & 3. Runde & 48 \\
\hline
\end{tabular}

Quelle: Leonhard Jörg, Rahel Falk, Evaluation of the Austrian Industrial Research Promotion Fund (FFF) and the Austrian Science Fund (FWF), Background report 3.1.2, FFF: Internal functioning and customer satisfaction, Technopolis, WIFO, Vienna, March 2004, www.ffg.at

Diese insgesamt 76 eingereichten Projekte stammen von 51 Impulszentren. Wenn wir als Richtschnur die vom Verband der Technologiezentren Österreichs (VTÖ) erfassten 103 Zentren heranziehen, dann kann man kann sagen, dass durch den Fördermodul REGplus mehr als die Hälfte der Zielgruppe erreicht wurde ${ }^{24}$. Diese 51 Zentren, die eingereicht haben, haben 36 Projekte gewonnen. Unter den 51 einreichenden Zentren befinden sich 6 so genannte 'virtuelle Impulszentren'. Die 36 genehmigten Projekte wurden allerdings ausschließlich von 'wirklichen' Impulszentren gewonnen. Die 'virtuellen Impulszentren' sind interessanter Weise leer ausgegangen. Wir werten dies auch als Hinweis darauf, dass während der Konzeptionsphase die Option 'Virtualität' etwas überschätzt wurde, was zweifellos auch Ausdruck des damals vorherrschenden Zeitgeists war.

Wir wollen unsere Aufmerksamkeit nun von der Betrachtung der Projekte auf die darin involvierten Geldmittel verlagern. Tabelle 11 gibt hierzu einen detaillierten Überblick. Es wurden über alle drei Runden Gesamtprojektkosten im Ausmaß von EUR 11.443.266 (AP) + EUR 22.078.530 (EP) = EUR 33.521.796 (gesamt) eingereicht. Die Projektvolumina der schließlich geförderten Projekte betrugen EUR 4.105.973 (AP) + EUR 13.740.828 (EP) = EUR 17.846.801 (gesamt). Die beantragten Förderungen belaufen sich auf EUR 3.578.372 (AP) + EUR 11.207.049 (EP) = EUR 15.195.421 (gesamt). Ausgeschüttet wurden schließlich nicht die beantragten Förderungen, sondern nur Teile davon, u.zw. EUR 3.255.354 (AP) (= 91\% der beantragten Förderung) + EUR 8.538.558 (= 76\% der beantragten Förderung) = EUR 11.793.912 (= 80\% der beantragten Förderung).

Es fällt auf, dass, abweichend von der maximalen Förderintensität von $80 \%$ (je zur Hälfte von Bund und Land zu leisten) in manchen Fällen die beantragten Fördervolumina den Anteil von 80\% überschreiten, z.B. in der zweiten Runde bei Aufbauprojekten in Höhe von 94\% bzw. bei Erweiterungsprojekten in Höhe von 85\%. Dies hat damit zu tun, dass die Möglichkeit eines so genannten Internationalisierungsbonus im

24 Die Zielgruppe ändert sich im Lauf der Zeit. So gab es zu den jeweiligen Einreichzeitpunkt ca. 60 Zentren (2001), in der zweiten Runde ca. 80 (2003) bzw. um die 100 (2005) (vgl. Tabelle 26). 
Ausmaß von max. EUR 50.000 je Projekt eingeräumt wurde. Rund ein Drittel der eingereichten Anträge (23 aus 76, entspricht EUR 976.880) hatten einen solchen Bonus vorgesehen, 12 der 36 geförderten Projekte (entspricht EUR 560.000) haben ihn schließlich erhalten.

Tabelle 11 Förderintensität nach Runden

\begin{tabular}{|c|c|c|c|c|}
\hline & 1. Runde & 2. Runde & 3. Runde & Alle Runden \\
\hline \multicolumn{5}{|l|}{ Aufbauprojekte } \\
\hline Projektkosten aller beantragten AP [EUR] & 4.123 .366 & 4.362 .083 & 2.957 .817 & 11.443 .266 \\
\hline Projektkosten aller geförderten AP [EUR] & 1.147 .689 & 2.072.146 & 886.138 & 4.105 .973 \\
\hline beantragte Förderung bei geförderten AP [EUR] & 918.150 & 1.951 .422 & 708.800 & 3.578 .372 \\
\hline beantragte Förderung bei geförderten AP [\%] & $80 \%$ & $94 \%$ & $80 \%$ & $87 \%$ \\
\hline gewährte Förderung bei geförderten AP [EUR] & 918.150 & 1.630 .844 & 706.360 & 3.255 .354 \\
\hline gewährte Förderung bei geförderten AP [\%] & $80 \%$ & $79 \%$ & $80 \%$ & $79 \%$ \\
\hline Anteil gewährter an beantragter Förderung AP [\%] & $100 \%$ & $84 \%$ & $99,7 \%$ & $91 \%$ \\
\hline Kürzung der beantragten Förderung AP [\%] & $0 \%$ & $16 \%$ & $0,3 \%$ & $9 \%$ \\
\hline \multicolumn{5}{|l|}{ Erweiterungsprojekte } \\
\hline Projektkosten aller beantragten EP [EUR] & 3.321 .738 & 10.978 .402 & 7.778 .390 & 22.078 .530 \\
\hline Projektkosten aller geförderten EP [EUR] & 2.682 .343 & 6.476 .267 & 4.582 .218 & 13.740 .828 \\
\hline beantragte Förderung bei geförderten EP [EUR] & 2.149 .338 & 5.517 .365 & 3.540 .346 & 11.207 .049 \\
\hline beantragte Förderung bei geförderten EP [\%] & $80 \%$ & $85 \%$ & $77 \%$ & $82 \%$ \\
\hline gewährte Förderung bei geförderten EP [EUR] & 1.913 .062 & 3.471 .338 & 3.154 .158 & 8.538 .558 \\
\hline gewährte Förderung bei geförderten EP [\%] & $71 \%$ & $54 \%$ & $69 \%$ & $62 \%$ \\
\hline Anteil gewährter an beantragter Förderung EP [\%] & $89 \%$ & $63 \%$ & $89 \%$ & $76 \%$ \\
\hline Kürzung der beantragten Förderung EP [\%] & $11 \%$ & $37 \%$ & $11 \%$ & $24 \%$ \\
\hline \multicolumn{5}{|l|}{ Alle Projekte } \\
\hline Projektkosten aller beantragten AP + EP [EUR] & 7.445 .104 & 15.340 .485 & 10.736 .207 & 33.521 .796 \\
\hline Projektkosten aller geförderten AP + EP [EUR] & 3.830 .032 & 8.548 .413 & 5.468 .356 & 17.846 .801 \\
\hline beantragte Förderung bei geförderten AP + EP [EUR] & 3.067 .488 & 7.468 .787 & 4.249 .146 & 14.785 .421 \\
\hline beantragte Förderung bei geförderten AP + EP [\%] & $80 \%$ & $87 \%$ & $78 \%$ & $83 \%$ \\
\hline gewährte Förderung bei geförderten AP + EP [EUR] & 2.831 .212 & 5.102 .182 & 3.860 .518 & 11.793 .912 \\
\hline gewährte Förderung bei geförderten AP + EP [\%] & $74 \%$ & $60 \%$ & $71 \%$ & $66 \%$ \\
\hline Anteil gewährter an beantragter Förderung AP + EP [\%] & $92 \%$ & $68 \%$ & $91 \%$ & $80 \%$ \\
\hline Kürzung der beantragten Förderung AP + EP [\%] & $8 \%$ & $32 \%$ & $9 \%$ & $20 \%$ \\
\hline
\end{tabular}

Quelle: FFG

\subsection{Angemessenheit der Projekte: Kürzungen und Auflagen}

Nicht alle der 76 eingereichten Projekte wurden gefördert, sondern nur 36 (47\%). Die Frage, ob Förderung oder nicht, war indes keine Entweder-oder-Entscheidung, vielmehr gab es immer wieder Kürzungen sowohl der förderbaren Kosten als auch, obgleich weniger häufig, der Förderintensität. Eine weiteres Charakteristikum der Förderansuchen im Rahmen der Förderlinie REGplus war das häufige Auftreten von Auflagen an die Förderungswerber. Wir wollen uns in diesem Kapitel der Frage von Kürzungen und Auflagen widmen, weil wir daraus wertvolle Einsichten in das Zusammenspiel von Programmzielen, Zielgruppen, Bewertungs- und Auswahlverfahren und wohl auch Kontext erhalten.

\subsubsection{Kürzungen}

Bei der Bewertung von Projekten gibt es einige grundsätzliche Fragestellungen, die in jedem Fall zu beantworten sind. Zunächst, ob sie grundsätzlich förderungswürdig sind, dann, ob die vorgesehenen Kosten und Aufwendungen anerkannt werden und schließlich wie hoch die Intensität der Förderung ist.

- Art und Inhalt. Im Zentrum steht hier die grundsätzliche Entscheidung, ob 
bestimmte kostenverursachende Tätigkeiten oder Aufwendungen überhaupt anzuerkennen sind oder nicht bestimmte Infrastrukturen, Kenntnisse und Fähigkeiten vorauszusetzen und daher nicht förderwürdig sind.

- Kosten. Soweit bestimmte Arten von Aufwendungen anerkannt werden, stellt sich Frage, inwieweit die damit verbundenen Kosten, die sich aus Umfang und Einheitskosten zusammen setzen, angemessen sind.

- Förderintensität. Sind diese Fragen soweit geklärt, ist schließlich die Frage nach der Förderquote, d.h. dem Umfang des Zuschussbedarfs zu bestimmen. Finanzkraft und Risikohöhe sind hier die zentralen Bestimmungsfaktoren.

In den bisher durchgeführten drei Runden ergibt sich ein durchaus unterschiedliches Verhalten, sowohl was die Art der Projekte - Aufbauprojekte vs. Erweiterungsprojekte - betrifft, als auch, wenn man nach den einzelnen Runden unterscheidet. Tabelle 12 liefert eine erste Übersicht.

Tabelle 12 Kürzung des beantragten Fördervolmens nach Runde und Projekttyp

\begin{tabular}{|l|c|c|c|}
\hline & Aufbauprojekte & Erweiterungsprojekte & Alle Projekte \\
\hline 1. Runde & $0 \%$ & $11 \%$ & $8 \%$ \\
\hline 2. Runde & $16 \%$ & $37 \%$ & $32 \%$ \\
\hline 3. Runde & $0,3 \%$ & $11 \%$ & $9 \%$ \\
\hline Alle Runden & $6 \%$ & $24 \%$ & $20 \%$ \\
\hline
\end{tabular}

Quelle: FFG

Bei Aufbauprojekten war die Jury mit Kürzungen der beantragten Förderungen im Ausmaß von durchschnittlich 6\% generell zurückhaltend, wohingegen Erweiterungsprojekte durchschnittlich um ein Viertel (24\%) gekürzt wurden. Besonders herausragend ist die 2. Ausschreibungsrunde, wo der Rotstift heftig angesetzt wurde und es zu Kürzungen um ein Drittel (32\%) kam. Besonders betroffen waren dabei die Erweiterungsprojekte mit 37\% Kürzungen der beantragten Förderung bei den im Prinzip geförderten Projekten.

In der 3. Runde gab es, ausgelöst durch die massiven Kürzungen in der zweiten Runde, ein Briefing durch die FFG an die Jurymitglieder, mit Kürzungen zurückhaltender umzugehen. Hauptargument war, dass durch die Kürzungen eine wesentliche Aufgabe, nämlich Vernetzungen, nicht mehr aufrecht erhalten werden können, insofern hier die Gefahr besteht, dass Projektpartner abspringen, weil ihr Eigenmittelanteil deutlich höher wird als der in der Absichtserklärung vereinbarte Anteil. Tabelle 13 gibt diese Intervention wieder, u.zw. sowohl beim Anteil der Projekte, bei denen Kürzungen vorgenommen wurden von $73 \%$ auf $42 \%$, als auch und vor allem beim Umfang der Kürzungen, der sich von 32\% der beantragten Förderungen auf 9\% verringerte. 
Tabelle 13 Kürzungen nach Runde und Projekttyp

\begin{tabular}{|l|c|c|c|c|c|c|c|c|c|c|}
\hline & \multicolumn{3}{|c|}{$\begin{array}{l}\text { geförderte } \\
\text { Projekte }\end{array}$} & \multicolumn{3}{c|}{$\begin{array}{c}\text { davon gekürzte } \\
\text { Projekte }\end{array}$} & \multicolumn{2}{c|}{$\begin{array}{c}\text { Umfang der Kürzungen } \\
\text { [\% der Zahl der Projekte] }\end{array}$} & $\begin{array}{c}\text { Umfang der Kürzungen } \\
\text { [\% der beantragten } \\
\text { Förderungen] }\end{array}$ \\
\hline & AP & EP & gesamt & AP & EP & gesamt & AP & EP & gesamt & \\
\hline 1. Runde & 5 & 4 & 9 & 0 & 2 & 2 & $0 \%$ & $50 \%$ & $22 \%$ & $8 \%$ \\
\hline 2. Runde & 7 & 8 & 15 & 3 & 8 & 11 & $43 \%$ & $100 \%$ & $73 \%$ & $32 \%$ \\
\hline 3. Runde & 4 & 8 & 12 & 0 & 5 & 5 & $0 \%$ & $63 \%$ & $42 \%$ & $9 \%$ \\
\hline Gesamt & 16 & 20 & 36 & 3 & 15 & 18 & $19 \%$ & $75 \%$ & $50 \%$ & $20 \%$ \\
\hline
\end{tabular}

Quelle: FFG

Tabelle 14 gibt eine Übersicht über die Art und Begründung der Kürzung. Dabei dominieren folgende Begründungen: Reduktion der förderbaren Kosten generell, Reduktion der Förderintensität sowie Streichung des Internationalisierungsbonus. Ein herausragender Fall ist dabei die Reduktion der beantragen Förderung eines Erweiterungsprojekts zur Errichtung eines Testlabors im Ausmaß von EUR 350.000 + EUR 50.000 (Internationalisierungsbonus) auf eine Machbarkeitsstudie im Ausmaß von EUR 20.000. Das Vorliegen der plausiblen Machbarkeitsstudie hat schließlich die Projektumsetzung im Rahmen von RIFupgrade ermöglicht.

Es lässt sich übrigens ein deutlicher Unterschied im Ausmaß der Reduktion der förderbaren Kosten bzw. der Förderintensität in Abhängigkeit von der Projektgröße beobachten. Die nicht gekürzten Projekte haben einen Umfang von durchschnittlich EUR 344.460 (beantragt), wohingegen die gekürzten Projekten deutlich größer sind, nämlich im Schnitt EUR 616.772 (beantragt). Wir führen dies auf mehrere Faktoren zurück, die im Einzelnen nicht immer säuberlich zu trennen sind. Gut separierbar ist die häufige Streichung des Internationalisierungsbonus, der zu oft aufgesetzt erschien. Ansonsten dominieren Kürzungen, die auf zu hohen Investitionsaufwand bzw. zu hohen (Personal)Aufwand im Verhältnis zu den vorgesehenen Aktivitäten abzielen. Bei Letzterem hat sich bei der Jury immer wieder die Vorstellung durchgesetzt, wonach bestimmte Aktivitäten entweder dem Regelbetrieb der Impulszentren zuzurechnen sind, als Kompetenz vorausgesetzt bzw. rascher erledigt werden können ${ }^{25}$. Generell vermuten wir, da bis auf wenige Projekte die Förderempfehlungen im Konsens ermittelt wurden, bei der Jury auch eine gewisse intuitive Vorstellung von der 'Angemessenheit' eines Projekts.

25 Diese Formulierungen lassen sich auch schärfer fassen: Immer wieder - im Zusammenhang mit Kürzungen - hatte die Jury einen gewissen Verdacht auf 'heiße Luft' und eine gewisse Gemächlichkeit, als auch das Hineinrechnen von Aktivitäten, die eigentlich vorausgesetzt werden sollten, also der Verdacht auf Mitnahmeverhalten. 
Tabelle 14 Kürzungen bei Aufbau- und Erweiterungsprojekten

\begin{tabular}{|c|c|c|c|c|c|c|c|}
\hline & & & $\begin{array}{l}\text { Gesamt- } \\
\text { volumen }\end{array}$ & $\begin{array}{l}\text { Investitions- } \\
\text { kosten }\end{array}$ & $\begin{array}{l}\text { Personal- } \\
\text { kosten }\end{array}$ & $\begin{array}{l}\text { Internationali- } \\
\text { sierungsbonus }\end{array}$ & $\begin{array}{c}\text { Förder- } \\
\text { intensität }\end{array}$ \\
\hline \multicolumn{8}{|c|}{ Aufbauprojekte } \\
\hline 1. Runde & & keine Kürzungen & & & & & \\
\hline \multirow[t]{4}{*}{ 2. Runde } & e-motion & Kürzung der Personalkosten um 10\% & & & $\mathrm{x}$ & & \\
\hline & GkmiT & $\begin{array}{l}\text { Internationalisierungsbonus nicht genehmigt, Kostenrahmen erscheint } \\
\text { generell zu hoch }\end{array}$ & $\mathrm{x}$ & & & $\mathrm{x}$ & \\
\hline & BIO.COM.NET & $\begin{array}{l}\text { Sach-, Investitions- und Drittkosten erscheinen überhöht, Kürzung dieser } \\
\text { Kosten um } 20 \%\end{array}$ & $\mathrm{x}$ & $\mathrm{x}$ & & & \\
\hline & Lantech & $\begin{array}{l}\text { Kürzung des Gesamtvolumens um } 10 \% \text {, Kostenrahmen erscheint generell } \\
\text { zu hoch }\end{array}$ & $\mathrm{x}$ & & & & \\
\hline 3. Runde & & keine Kürzungen & & & & & \\
\hline \multicolumn{8}{|c|}{ Erweiterungsprojekte } \\
\hline \multirow[t]{2}{*}{ 1. Runde } & innonet B & Kostenreduktion von mind. $20 \%$ & $\mathrm{x}$ & & & & \\
\hline & LUHIZ & $\begin{array}{l}\text { Kürzung um EUR } 58.140 \text { (investive Förderung soll nicht über } 33 \text { \% } \\
\text { liegen) }\end{array}$ & & $\mathrm{x}$ & & & \\
\hline \multirow[t]{7}{*}{ 2. Runde } & IR Süd Ost & Kürzung der Personalkosten um 10\% & & & $\mathrm{x}$ & & \\
\hline & $\mathrm{B}^{2}$ - Next Step & $\begin{array}{l}\text { Kürzung der Förderquote von } 80 \% \text { auf } 70 \% \text {, Kürzung der } \\
\text { Gesamtprojektkosten um EUR } 273.453\end{array}$ & $\mathrm{x}$ & & & & $\mathrm{x}$ \\
\hline & Sell-it & $\begin{array}{l}\text { 187.424 € / Internationalisierungsbonus nicht genehmigt, Reduktion des } \\
\text { Gesamtvolumens um } 20 \%\end{array}$ & $\mathrm{x}$ & & & $\mathrm{x}$ & \\
\hline & PROOF & $\begin{array}{l}\text { Reduktion des Gesamtantrags von EUR } 875.000 \text { auf EUR 50.000, um den } \\
\text { ersten, im Gesamtprojekt vorgesehenen Schritt, eine Machbarkeitsstudie } \\
\text { zu erstellen, durchzuführen }\end{array}$ & $\mathrm{x}$ & & & & \\
\hline & EssenZ & $\begin{array}{l}\text { Internationalisierungsbonus nicht genehmigt, Reduktion der Förderquote } \\
\text { für den zugekauften F\&E-Teils auf 50\% }\end{array}$ & & & & $\mathrm{x}$ & $\mathrm{x}$ \\
\hline & RWB Steyr & $\begin{array}{l}\text { Gesamtvolumen erscheint im Verhältnis zu den geplanten Aktivitäten } \\
\text { überhöht, Kürzung um } 25 \%\end{array}$ & $\mathrm{x}$ & & & & \\
\hline & INCUBAT & $\begin{array}{l}\text { Internationalisierungsbonus nicht genehmigt, Reduktion der Förderquote } \\
\text { von } 80 \% \text { auf } 70 \%\end{array}$ & & & & $\mathrm{x}$ & $\mathrm{x}$ \\
\hline
\end{tabular}




\begin{tabular}{|c|c|c|c|c|c|c|c|}
\hline & & & $\begin{array}{l}\text { Gesamt- } \\
\text { volumen }\end{array}$ & $\begin{array}{l}\text { Investitions- } \\
\text { kosten }\end{array}$ & $\begin{array}{l}\text { Personal- } \\
\text { kosten }\end{array}$ & $\begin{array}{l}\text { Internationali- } \\
\text { sierungsbonus }\end{array}$ & $\begin{array}{c}\text { Förder- } \\
\text { intensität }\end{array}$ \\
\hline & NIMM 2 & $\begin{array}{l}\text { Internationalisierungsbonus nicht genehmigt, Kürzung des Gesamtvolu- } \\
\text { mens um 30\%, da nicht unbedingt erforderliche Maßnahmen im Projekt } \\
\text { geplant, insbesondere wird auf bekannte, ausgetestete Tools zurückgegrif- } \\
\text { fen; zu hoher Zeitaufwand kalkuliert, Kürzung der Förderquote von } 80 \% \\
\text { auf 70\%, weil mit Rückflüssen aus der Wirtschaft zu rechnen ist }\end{array}$ & $\mathrm{x}$ & & & $\mathrm{x}$ & $\mathrm{x}$ \\
\hline \multirow[t]{7}{*}{ 3. Runde } & i-weg & Erhöhung des Eigenmittelanteils auf mindestens 10\% & & & & & $\mathrm{x}$ \\
\hline & PEC & Erhöhung des Eigenmittelanteils auf mindestens 10\% & & & & & $\mathrm{x}$ \\
\hline & bio.com.net II & $\begin{array}{l}\text { Reduktion der bundesseitige Förderungsintensität von } 40 \% \text { auf } 30 \% \text { und } \\
\text { Darstellung in einem überarbeiteten Kosten- und Finanzierungsplans (bei } \\
\text { gleich bleibenden Gesamtkosten und gleich bleibenden durchzuführenden } \\
\text { Arbeitspaketen) }\end{array}$ & & & & & $\mathrm{x}$ \\
\hline & $\begin{array}{l}\text { hyper metall } \\
\text { processing }\end{array}$ & $\begin{array}{l}\text { Streichung der Kosten des Kunststoffclusters aus den förderbaren } \\
\text { Gesamtkosten (bei gleich bleibenden durchzuführenden Arbeitspaketen) } \\
\text { d.h. Reduktion der förderbaren Gesamtkosten von } 409.450 \text { EUR auf } \\
\text { 329.550 EUR }\end{array}$ & $\mathrm{X}$ & & & & \\
\hline & energycenter-net & $\begin{array}{l}\text { Anpassung des Tagsatzes an die Höchstgrenze } 750 \text { EUR und Korrektur } \\
\text { der Kostenplanung. Die bundesseitige Förderungsintensität ist von } 40 \% \\
\text { auf } 30 \% \text { der förderbaren Kosten (auf Basis des korrigierten Kostenplans) } \\
\text { zu reduzieren und in einem überarbeiteten Kosten und Finanzierungsplan } \\
\text { darzustellen (bei gleich bleibenden Gesamtkosten und gleich bleibenden } \\
\text { durchzuführenden Arbeitspaketen) }\end{array}$ & & & $\mathrm{x}$ & & $\mathrm{x}$ \\
\hline & FH Region OÖ & $\begin{array}{l}\text { Reduktion der Bundesförderung auf max.150.000 EUR bei gleichzeitiger } \\
\text { Umwandlung des Erweiterungsprojekts zu einem Aufbauprojekt }\end{array}$ & & & & & $\mathrm{x}$ \\
\hline & LANTECH2 & Streichung des Internationalisierungsbonus & & & & $\mathrm{x}$ & \\
\hline
\end{tabular}

Quelle: Juryprotokolle der jeweiligen Ausschreibungsrunden, FFG 


\subsubsection{Auflagen bei Aufbau- und Erweiterungsprojekten}

Projekte aus dem RIF 2000 Programm (also REGplus und RIFupgrade) sind auf den ersten Blick an eine sehr homogene Zielgruppe gerichtet, nämlich Technologie-, Innovations-, Gründer- oder Impulszentren. Tatsächlich trügt der Schein. Auf den zweiten Blick sind sie ausgesprochen inhomogen. Sie weisen unterschiedliche Biographien, unterschiedliche Eigentumsverhältnisse, unterschiedliche Belegungen, nicht zuletzt unterschiedliche Managementkapazitäten und -fähigkeiten auf. Nicht minder relevant ist das Umfeld, in dem die einzelnen Zentren agieren ${ }^{26}$. Ein weiterer Aspekt, der ins Treffen zu führen ist, betrifft die Neuheit dieser Art der Förderung: Nachdem über längere Zeit Errichtung und Betrieb von Gebäuden, Aufbau von Infrastruktur und Vermietung der Gebäude im Vordergrund gestanden sind, geht es nunmehr darum, Vernetzung unter den Unternehmen sowie mit spezialisierten Institutionen bzw. überhaupt Regionalentwicklung voranzutreiben. Gibt es unter diesen heterogenen Bedingungen bzw. sich innerhalb einer kurzen Zeitspanne deutlich verändernden Anforderungen eine klare Vorstellung, was ein gut funktionierendes Impulszentrum ist, näherhin was 'gute' Projekte im Sinne des Programms und damit seiner Zielsetzungen, Verfahrensweisen und deren praktischer Handhabung sind?

Ein Blick in die nachfolgenden Tabellen gibt Stoff für eine ganze Reihe von Betrachtungen mit teils überraschenden Resultaten. Nehmen wir zu Überblickszwecken Tabelle 15, wo dargestellt ist, bei welchen Projekten der jeweiligen Runden von der Jury Auflagen in Bezug auf Reduktionen, Präzisierungen und Vernetzungen (mit anderen Projekten bzw. Institutionen) gemacht wurden sowie die umfangreichere Tabelle 17, die die materiellen Vorschläge wiedergibt, so zeigt sich ein recht deutliches Bild.

- 3 von 36 förderungswürdigen Projekten werden ohne Auflage zur Förderung vorgeschlagen.

- Bei 21 von 36 Projekten (58\%) wird eine Reduktion der förderbaren Kosten bzw. der Förderintensität vorgeschlagen.

- 3 von 36 förderungswürdigen Projekte sind unpräzise beschrieben und müssen nachgebessert werden.

- Bei etwas mehr als der Hälfte der förderungswürdigen Projekte (53\%) wird eine Vernetzung mit anderen Projekten bzw. Institutionen vorgeschlagen bzw. eingefordert. $^{27}$

- Die drei Projekte ohne Auflage werden in der ersten Ausschreibungsrunde genehmigt. Ab der zweiten Ausschreibungsrunde werden überhaupt keine Projekte mehr zur Förderung vorgeschlagen, die nicht irgendeinen Nachbesserungs- bzw. Änderungsbedarf aufweisen.

- Genau ein Viertel aller förderungswürdigen Projekte haben drei, fast die Hälfte (47\%) immerhin zwei Auflagen, 17\% eine Auflage.

26 Wir kommen auf dieses Thema weiter unten unter dem Stichwort 'Projektportfolio' noch einmal zu sprechen (Kapitel 5.2).

27 Wir interpretieren diese hohe Quote als einen Indikator dafür, dass seitens der Jury ein hohes Bewusstsein für die Existenz von thematisch verwandten Programmen und den entsprechenden Interferenzen mit dem REGplus Programm vorliegt. 
Tabelle 15 Auflagen an geförderte Projekte: Reduktionen, Präzisierungen, Vernetzungen

\begin{tabular}{|c|c|c|c|c|}
\hline Runde & Projekt & Reduktion & Präzisierung & Vernetzung \\
\hline \multirow[t]{9}{*}{ 1. Runde } & tzs.net & & & \\
\hline & NIMM & & & \\
\hline & AROMA & & $\mathrm{x}$ & \\
\hline & LEBRING & & $\mathrm{x}$ & $\mathrm{x}$ \\
\hline & IT-LAB & & & \\
\hline & WTZ & & $\mathrm{x}$ & \\
\hline & innonet B & $\mathrm{x}$ & $\mathrm{x}$ & $\mathrm{x}$ \\
\hline & LUHIZ & $\mathrm{x}$ & & \\
\hline & CNC-Inet & & & \\
\hline \multirow{15}{*}{ 2. Runde } & ENERMODE & & $\mathrm{x}$ & $\mathrm{x}$ \\
\hline & e-motion & $\mathrm{x}$ & & $\mathrm{x}$ \\
\hline & IR Süd Ost & $\mathrm{x}$ & $\mathrm{x}$ & \\
\hline & $\mathrm{B}^{2}$ - Next Step & $\mathrm{x}$ & & $\mathrm{x}$ \\
\hline & K3 & & $\mathrm{x}$ & \\
\hline & Sell-it & $\mathrm{x}$ & & $\mathrm{X}$ \\
\hline & PROOF & $\mathrm{x}$ & & \\
\hline & EssenZ & $\mathrm{x}$ & $\mathrm{x}$ & $\mathrm{x}$ \\
\hline & GKmit & $\mathrm{x}$ & $\mathrm{x}$ & $\mathrm{x}$ \\
\hline & RWB Steyr & $\mathrm{x}$ & $\mathrm{x}$ & \\
\hline & water-tec-net & & $\mathrm{x}$ & $\mathrm{x}$ \\
\hline & BIO.COM.NET & $\mathrm{x}$ & $\mathrm{x}$ & \\
\hline & Lantech & $\mathrm{x}$ & $\mathrm{x}$ & $\mathrm{x}$ \\
\hline & INCUBAT & $\mathrm{x}$ & $\mathrm{x}$ & $\mathrm{x}$ \\
\hline & NIMM 2 & $\mathrm{x}$ & $\mathrm{x}$ & \\
\hline \multirow[t]{12}{*}{ 3. Runde } & TRIS & & $\mathrm{x}$ & $\mathrm{x}$ \\
\hline & AREAmp3 & & $\mathrm{x}$ & $\mathrm{x}$ \\
\hline & LANTECH2 & & $\mathrm{x}$ & $\mathrm{x}$ \\
\hline & PEC & $\mathrm{x}$ & $\mathrm{x}$ & \\
\hline & rapidPRO & & $\mathrm{x}$ & \\
\hline & GDI Steyr & $\mathrm{x}$ & $\mathrm{x}$ & $\mathrm{x}$ \\
\hline & bio.com.net II & $\mathrm{X}$ & $\mathrm{x}$ & \\
\hline & hyper metall processing & $\mathrm{x}$ & $\mathrm{x}$ & $\mathrm{x}$ \\
\hline & TRANSFER & & $\mathrm{x}$ & $\mathrm{x}$ \\
\hline & i-weg & $\mathrm{x}$ & $\mathrm{x}$ & $\mathrm{x}$ \\
\hline & energycenter-net & $\mathrm{x}$ & $\mathrm{x}$ & $\mathrm{x}$ \\
\hline & FH Region OÖ & $\mathrm{x}$ & $\mathrm{x}$ & \\
\hline 1. Runde & & $22 \%$ & $44 \%$ & $22 \%$ \\
\hline 2. Runde & & $80 \%$ & $73 \%$ & $60 \%$ \\
\hline 3. Runde & & $58 \%$ & $100 \%$ & $67 \%$ \\
\hline Gesamt & & $58 \%$ & $75 \%$ & $53 \%$ \\
\hline
\end{tabular}

Quelle: Juryprotokolle der jeweiligen Ausschreibungsrunden, FFG

Es herrscht, diesen Schluss kann man angesichts diese Faktenlage getrost ziehen, auch unter den im Prinzip förderungswürdigen Projekten seitens der Impulszentren ein hoher Grad an Unklarheit darüber, was ein gutes Projekt ausmacht. Wir können diesen Befund noch zuspitzen: 3 aus 76 eingereichten Projekten, das sind 4\%, haben einen tadellosen Projektantrag eingereicht. 8\% wurden mit einer Auflage, 22\% mit zwei und 12\% mit drei Auflagen schließlich doch gefördert, 53\% lagen außerhalb der Förderwürdigkeit.

Wenn die Wahrscheinlichkeit eines tadellosen Antrags so gering ist, könnte dann 
nicht das Problem nicht nur bei den Antragstellern, also den Impulszentren liegen, sondern auch beim Programm selbst? Wir wollen dieser Frage im Folgenden nachgehen. Die Förderabwicklungsstelle hat im Zuge der Projektbegutachtung von den einzelnen Jurymitgliedern eine schriftliche Bewertung der Projektanträge vorab eingeholt. Jedes Jurymitglied musste neben einer qualitativen verbalen Bewertung auch eine summarische Note auf der Skala 0-4 (mit 4 als Grad der höchsten Förderwürdigkeit) vergeben. Sinn und Zweck dieses Verfahrensschritts war es, eine Vorsortierung der einzelnen Projektanträge zu erhalten um die Sitzung der Jury effizient zu gestalten und den größeren Teil der knappen Zeit den strittigen Fälle zu widmen ${ }^{28}$.

Hierzu liegen die einzelnen Einschätzungen aus der zweiten und dritten Ausschreibungsrunde vor, also von 53 von insgesamt 76 Projektanträgen. Wir haben als Maß für die unzweideutige Interpretation der Programmziele einschließlich ihrer Anwendung auf die vorliegenden Projektanträge die Abweichung zwischen der besten und der schlechtesten Benotung herangezogen. Tabelle 16 liefert die Ergebnisse. Hier stellt sich etwas ganz Erstaunliches heraus: In 25\% der Anträge gibt es sowohl die beste $(=4)$ als auch die schlechteste $(=0)$ Note. In weiteren 34\% der Fälle beläuft sich der Abstand immerhin auf drei Stufen in der Notenskala. In 59\% weichen also die individuellen Einschätzungen der Jurymitglieder erheblich voneinander ab.

Tabelle 16 Abweichungen der Vorab-Bewertungen durch die Jury

\begin{tabular}{|l|c|c|c|c|c|}
\hline & \multicolumn{5}{|c|}{ Abweichungen [Punkte] } \\
\hline Zahl der geförderten Projekte & 0 & 1 & 2 & 3 & 4 \\
\hline Zahl der nicht förderten Projekte & 2 & 4 & 6 & 10 & 5 \\
\hline Gesamt & 1 & 1 & 8 & 8 & 8 \\
\hline & 3 & 5 & 14 & 18 & 13 \\
\hline
\end{tabular}

Quelle: FFG

NB: Die Abweichungen errechnen sich als Differenz zwischen dem Maximum und dem Minimum der von den einzelnen Jurymitglieder vorab vergebenen Punkte. Grundlage waren die 2. und 3.

Ausschreibungsrunde, das sind 53 von 76 Projekten.

Wir betrachten diese Divergenz als einen starken Hinweis darauf, dass nicht nur die Zielgruppe des Programms, also die Impulszentren, sondern auch die Programmträger, hier repräsentiert durch die de facto Entscheidungsinstanz, nämlich die Jury, ein ausgesprochen heterogenes Bild von REGplus haben.

Man sollte meinen, dass hier ein ernsthaftes Problem vorliegt. Immerhin treffen die meisten Antragsteller daneben, und mehr noch, die Entscheidungsträger sind sich hochgradig uneins darüber, was überhaupt ein förderwürdiges Projekt ist. Wir wollen uns indes einer solchen Kritik nicht anschließen, zunächst aus dem Grund, weil hier ein unvollständiges Bild des Geschehens vorliegt.

Zunächst wird nicht so heiß gegessen, wie gekocht wird: Jene Förderempfänger, die Projekte sowohl gewonnen als auch verloren haben, und die also beide Sorten von Gefühlen kennen, stimmen bis auf wenige, dort allerdings hart verteidigte Ausnahmen darin überein, dass jene Anträge, die sie gewonnen haben auch vor ihrer inneren Instanz die höhere Wertschätzung genießen im Vergleich zu jenen, die sie schließlich

28 Ein legitimes Nebenmotiv bestand zweifellos auch darin, sicherzustellen, dass die Jurymitglieder vor der Sitzung die Dokumente auch sorgfältig studiert hatten. 
verloren haben. Wir interpretieren diese 'innere Kohärenz' als die äußere Hülle des Konfliktraums.

Unser Hauptargument, mit dem wir die Bedeutung des soweit beobachtbaren Dissens abschwächen wollen, bezieht seine Geltung aus einer ganz anderen Quelle, daraus nämlich, dass, bis auf wenige Fälle, die Juryentscheidungen einstimmig waren. Der Prozess der Entscheidungsfindung wird also erst durch die beiden Verfahrensschritte vollständig, die Vorab-Bewertung durch die Jurymitglieder und die Diskussion in der Jurysitzung. Man kann und muss aber die Frage stellen, wie es kommen kann, dass sich ein veritabler Dissens innerhalb von durchschnittlich 15 Minuten Diskussion in einen Konsens verwandeln kann. Der Unterschied, der diese beiden Verfahrensschritte zu einem Ganzen zusammen führt, liegt vor allem darin, dass in der Vorab-Bewertung in der Regel relativ wenig Information über den Kontext der Projekte vorliegt bzw. berücksichtigt wird, wohingegen während der Jurysitzung vermehrt Kontextfaktoren ins Spiel gebracht werden. Gerade dies zu leisten, ist eine wesentliche Qualität dieses kombinierten Verfahrens, aber auch der Qualität der Jurymitglieder. Es ist offenbar gelungen, die Jurymitglieder so auszuwählen, dass eine günstige Kombination aus inhaltlicher und kontextbezogener Beurteilung möglich war.

Für künftige Programme dieser Art sollte in Bezug auf die Organisation der Entscheidungsfindung jedenfalls an einer starken Jury festgehalten werden. Zugleich, und diese Empfehlung gilt unbeschadet aller anderen Überlegungen, wird es in Zukunft wichtiger denn je sein, das zu fördernde Projekt stets als Mittel zum Zweck zu betrachten. Dies macht es wichtiger denn je, im Zuge des Förderansuchens eine ausführliche Darstellung des Kontexts und damit der Ausgangssituation einzufordern. Das Projekt und seine Förderwürdigkeit sind daher vor allem im Lichte der jeweiligen Ausgangssituation und der anzustrebenden Ziele zu würdigen, und weniger aufgrund seiner internen Charakteristika. Dies ändert freilich nichts daran, das Programm sorgfältig zu kommunizieren und auf entsprechendes Feedback zwischen Förderer und Förderempfänger zu achten. 
Tabelle 17 Auflagen bei Aufbau- und Erweiterungsprojekten

\begin{tabular}{|c|c|c|}
\hline Runde & Projekt & Auflagen \\
\hline \multirow[t]{9}{*}{ 1. Runde } & tzs.net & - $\quad$ keine Auflagen \\
\hline & NIMM & keine Auflagen \\
\hline & AROMA & - $\quad$ Bessere Darstellung der Verschränkung des Forschungsteils mit Wirtschaftlichkeit und Vermarktung erforderlich \\
\hline & LEBRING & $\begin{array}{ll}- & \text { Kooperationsvereinbarung mit der HTBLA ist als 1. Meilenstein Ende } 2001 \text { nachzuweisen } \\
- & \text { Umsetzungsschritte vorweisen bezüglich Technologieachse Graz-Marburg als 1. Meilenstein Ende } 2001 \\
- & \text { Zeit- und Arbeitsplan exakter darstellen } \\
\end{array}$ \\
\hline & IT-LAB & \\
\hline & WTZ & - $\quad$ Mitteleinsatz ist noch detaillierter darzustellen \\
\hline & innonet B & $\begin{array}{ll}- & \text { Kostenreduktion von mind. } 20 \% \text { (Bereich Start Smart) } \\
- & \text { Detaillierter Kostennachweis (Sachkosten, Drittkosten) } \\
- & \text { Zusätzliche Einbindung von mind. } 2 \text { innovativen Unternehmen erforderlich (Privatunternehmen, die im Wettbewerb stehen) } \\
\end{array}$ \\
\hline & LUHIZ & - $\quad$ Kürzung um EUR 58.140 (investive Förderung soll nicht über 33 \% liegen) \\
\hline & CNC-Inet & $-\quad$ keine Auflagen \\
\hline \multirow[t]{4}{*}{ 2. Runde } & ENERMODE & $\begin{array}{ll}- & \text { Bestandsaufnahme der derzeitigen Energiebilanz im ersten Halbjahr der Projektlaufzeit } \\
- & \text { Konkretisierung wie die geplanten Maßnahmen im Ennstal positive Wirkungen für das TDZ und die umgebende Region erzeugen - Beitrag zur regionalen } \\
& \text { Wertschöpfung } \\
- & \text { Empfehlung: Einbeziehung des oberösterreichischen Öko-Energieclusters } \\
\end{array}$ \\
\hline & e-motion & $\begin{array}{ll}- & \text { Die Anzahl von einbezogenen, anwendenden Unternehmen, die im gewählten Schwerpunktbereich tätig sind, ist auf zumindest } 5 \text { zu erhöhen und im Zuge } \\
& \text { des Projektes nach einjähriger Laufzeit nachzuweisen } \\
- & \text { Kürzung der Personalkosten um } 10 \% \\
- & \text { Vernetzung mit weiteren Impulszentren und Initiativen, insb. mit dem Mechatronik-Cluster } \\
- & \text { Empfehlung: Coaching des Projektteams }\end{array}$ \\
\hline & IR Süd Ost & $\begin{array}{ll}\text { - } & \text { Meilensteine im Sinne von Quantifizierung der Projektauswirkungen sind zu definieren, insbesondere die Auswirkungen auf wirtschaftlich umsetzbare } \\
& \text { Ergebnisse } \\
\text { - } & \text { Verantwortungsbereich der angeführten Personen sind weiter zu spezifizieren, ein stringentes Projektmanagement ist sicherzustellen } \\
- & \text { Kürzung der Personalkosten um } 10 \%\end{array}$ \\
\hline & $\mathrm{B}^{2}$ - Next Step & $\begin{array}{ll}- & \text { Kürzung der Förderquote von } 80 \% \text { auf } 70 \% \\
- & \text { Kürzung der Gesamtprojektkosten um } 273.453 € \text {, um den geforderten Eigenmittelanteil von } 10 \% \text { zu erreichen (TZ-Beiträge herausgerechnet) Zudem } \\
& \text { erscheint die angeführte Zahl der Personentage für die geplanten Aktivitäten systematisch zu hoch angesetzt } \\
- & \text { Quantitativer Nachweis der Projektauswirkungen zu Meilensteinen } \\
- & \text { Abgrenzung zur Finanzierung 'start smart' } \\
- & \text { Empfehlung: Kooperation mit benachbarten Bundesländern }\end{array}$ \\
\hline
\end{tabular}




\begin{tabular}{|c|c|c|}
\hline Runde & Projekt & Auflagen \\
\hline & K3 & $\begin{array}{ll}- & \text { Präzisierung der angestrebten Kooperationsbeziehungen } \\
- & \text { Sichtbarmachung der Regionalisierung der Forschungsaktivitäten mit MUL und TGM als best practice } \\
\end{array}$ \\
\hline & Sell-it & $\begin{array}{ll}- & \text { Eigenmittelanteil liegt nicht bei 10\%, tech2B ist kein Unternehmen, sein Beitrag kann deshalb nicht den } 10 \% \text { Unternehmensbeiträgen zugerechnet } \\
\text { werden. } & \\
\text { - } & \text { Eigenmittelanteil muss bei } 10 \% \text { liegen. Das bedeutet eine Reduzierung des Gesamtprojekts auf } 585.700 € \text {. Reduktion des Gesamtvolumens um } 20 \% . \\
- & \text { Stärkere Einbeziehung von M\&V Unterstützungsaktivitäten, oö. Clusterinitiativen und von vorhandenem Beratungs-Know how (z.B. CATT, TIM, } \\
& \text {...).Zahl der Modellanwender-Unternehmen (S.18) ist von } 5 \text { auf } 10 \text { zu erhöhen }\end{array}$ \\
\hline & PROOF & $\begin{array}{l}\text { - } \begin{array}{l}\text { Das Projekt erfordert die Erstellung einer Machbarkeitsanalyse, in der die Implikationen für die Region und für das Impulszentrum ausgewiesen sind. } \\
\text { Deshalb ist jener Teil des Förderungsansuchens, der das Vorprojekt beschreibt, detailliert darzustellen. Bei der Erstellung des Vorprojekts sind externe } \\
\text { Gutachter einzubeziehen. Gesamtprojektkosten: max. } 50.000 €\end{array} \\
\end{array}$ \\
\hline & EssenZ & $\begin{array}{ll}- & \text { Reduktion der Förderquote für den zugekauften F\&E-Teils (JR) auf 50\% } \\
- & \text { Erläuterung, was im vorigen Projekt bisher erreicht worden ist und wie die Vorarbeiten aus Aroma in EssenZ einfließen (z.B. Versuchsanbau) } \\
- & \text { Aufteilung der Verantwortung im Förderungsnehmerkonsortium } \\
- & \text { Sicherstellung der Kooperation zwischen den Projektträgern durch entsprechende Verträge } \\
- & \text { Empfehlung: Coaching für die beiden Projektleiter } \\
\end{array}$ \\
\hline & GKmit & $\begin{array}{ll}- & \text { Kostenrahmen erscheint hoch, verschiedene Kostenpositionen sind zu reduzieren, daher Kürzung der Gesamtprojektkosten (ohne Internationalisie- } \\
& \text { rungsbonus) um } 10 \% \\
- & \text { Darstellung der Kunststoffkompetenz des Teams } \\
- & \text { Kostenplan (insb. Drittkosten), Personal- und Ressourcenplan aufgliedern, Tagsätze für Projektassistenz sind zu hoch } \\
- & \text { Detaillierte und übersichtliche Darstellung der Arbeitspakete und kostenmäßige Zuordnung } \\
- & \text { Klare Abstimmung mit den sonstigen Vorhaben des Landes NÖ unter Einschluss von ecoplus / Land NÖ (insb. WST 2, WST 3) } \\
- & \text { Empfehlung: Kooperation mit dem OÖ Kunststoffcluster }\end{array}$ \\
\hline & RWB Steyr & $\begin{array}{ll}- & \text { Gesamtvolumen erscheint im Verhältnis zu den geplanten Aktivitäten überhöht, Kürzung um 25\% } \\
\text { - } & \text { Darstellung, welche Nachfrage nach den Projektergebnissen besteht } \\
\text { - } & \text { Darstellung der erwarteten Auswirkungen des Projekts } \\
\end{array}$ \\
\hline & water-tec-net & $\begin{array}{ll}- & \text { Klarere Darstellung der angesprochenen konkreten Technologien und der damit verbundenen Zielrichtung des Projektes } \\
\text { - } & \text { Stärkeres Commitment der beteiligten Firmen, insbesondere der BWT als Leitbetrieb }\end{array}$ \\
\hline & BIO.COM.NET & $\begin{array}{ll}- & \text { Sach-, Investitions- und Drittkosten erscheinen überhöht, Kürzung dieser Kosten um 20\% } \\
- & \text { Aufschlüsselung und Begründung der Sachkosten, insbesondere der Reisekosten (Begründung der hohen Kosten von int. Vernetzung, } \\
& \text { Fachveranstaltungen, Besuch Life Science Standorte etc.) } \\
- & \text { Detaillierte Darstellung einer Aufteilung des Budgets auf die Projektpartner } \\
\end{array}$ \\
\hline & Lantech & $\begin{array}{ll}- & \text { Kürzung des Gesamtvolumens um } 10 \% \text {, Kostenrahmen erscheint generell zu hoch Konkretisierung der Projektinhalte und der Umsetzung } \\
- & \text { Detaillierte Begründung der Sachkosten (z.B. 'best practise' - Kosten von } 25.000 € \text { ) } \\
- & \text { Darstellung, wie mit dem Projekt der inhaltliche Schwerpunkt des Zentrums gestärkt werden kann } \\
- & \text { Empfehlung: Coaching / Supervision }\end{array}$ \\
\hline
\end{tabular}




\begin{tabular}{|c|c|c|}
\hline Runde & Projekt & Auflagen \\
\hline & INCUBAT & $\begin{array}{ll}- & \text { Reduktion der Förderungsquote von } 80 \% \text { auf } 70 \% \\
- & \text { Die Förderung kann für den zweiten Teil der Laufzeit reduziert werden, wenn die angestrebte Anzahl an technologieorientierten Gründungen bis zur } \\
& \text { Hälfte der Laufzeit nicht erreicht wird } \\
- & \text { Darstellung der geplanten wirtschaftlichen Weiterentwicklung des Inkubators nach Auslaufen der REGplus-Förderung (Darstellung der geplanten } \\
& \text { künftigen Finanzierungsstruktur) } \\
- & \text { Empfehlung: Konkretisierung der Kooperation mit Forschungs- und Bildungseinrichtungen, insb. FH Dornbirn }\end{array}$ \\
\hline & NIMM 2 & $\begin{array}{ll}\text { - } & \text { Kürzung des Gesamtvolumens um } 30 \% \text {, da nicht unbedingt erforderliche Maßnahmen im Projekt geplant, insbesondere wird auf bekannte, ausgetestete } \\
& \text { Tools zurückgegriffen; zu hoher Zeitaufwand kalkuliert } \\
\text { - } & \text { Kürzung der Förderquote von } 80 \% \text { auf } 70 \% \text {, weil mit Rückflüssen aus der Wirtschaft zu rechnen ist } \\
- & \text { Deckelung der Tagsätze mit } 750 € \\
- & \text { Detaillierte Darstellung der für dieses Projekt relevanten Projektergebnisse aus NIMM 1. Worauf wird konkret aufgebaut? } \\
- & \text { externe Projektevaluierung ist ins Projekt mit einzubeziehen, Darstellung der Projektauswirkungen } \\
\end{array}$ \\
\hline \multirow[t]{5}{*}{ 3. Runde } & TRIS & $\begin{array}{lll}- & \text { Vorlage einer Absichtserklärung zur Ko-Finanzierung seitens des Landes Steiermark innerhalb } 14 \text { Tagen } \\
- & \text { Einbeziehung und Darstellung kompetenter externer ExpertInnen (Uni/FH) } \\
- & \text { Detaillierte Definition von Arbeitspaketen, Arbeitsteilung und Meilensteinen } \\
\end{array}$ \\
\hline & AREAmp3 & $\begin{array}{ll}- & \text { Vorlage einer Absichtserklärung zur Ko-Finanzierung seitens des Landes Steiermark (bzw. Gemeindebund) innerhalb einer Frist von } 14 \text { Tagen } \\
- & \text { Nachvollziehbare Zuordnung der Ressourcen zu den einzelnen dargestellten Arbeitspaketen mit Definition konkreter Meilensteine } \\
- & \text { Überarbeitung bzw. höherer Detaillierungsgrad des Personal- und Sachkostenplans } \\
- & \text { Abstimmung bzw. Kooperation mit dem Materialcluster } \\
\end{array}$ \\
\hline & LANTECH2 & $\begin{array}{ll}- & \text { Überarbeitung des Projektplanes mit detaillierter Arbeitsteilung zwischen des Projektpartnern } \\
\text { - } & \text { Definition eines Milestone mit Stop or Go Entscheidung nach } 6 \text { Monaten (Zwischenbericht mit folgenden Darstellungen): Akquisition der Partner für das } \\
& \text { Chancenkapital } \\
\text { - } & \text { Abstimmung Meinungsbildnern und Leitbetrieben } \\
- & \text { Recherche vorhandener Projekte Reflexion mit ExpertInnen. Die Förderung des Projektes ist keinesfalls Grundlage für eine Förderung einer zweiten } \\
& \text { Ausbaustufe }\end{array}$ \\
\hline & PEC & $\begin{array}{ll}- & \text { Erhöhung des Eigenmittelanteils auf mind. 10\% } \\
- & \text { Darstellung eines nicht mit AplusB geförderten Eigenmittelanteils sowie der Personalkosten des ZAT aus dem ungeförderten Bereich des ZAT (d.h. aus } \\
& \text { dem Non-AplusB Bereich) } \\
- & \text { Darstellung des geplanten Outputs aus den Leistungspaketen } \\
- & \text { Darstellung zur Sicherung/Verwertung von IPR } \\
\end{array}$ \\
\hline & rapidPRO & $\begin{array}{ll}- & \text { Stop or Go Entscheidung nach AP1 (Definition, Konzept, Marktpotential) } \\
\text { - } & \text { Detaillierung der Investitionskosten sowie Sach- und Drittkosten } \\
\text { - } & \text { Genaue Darstellung der Ausbildungsaspekte und des Technologietransfers an relevante KMU der Region sowie der Wirkungen des Projektes auf die } \\
& \text { Managementkompetenz des Technologiezentrums }\end{array}$ \\
\hline
\end{tabular}




\begin{tabular}{|c|c|c|}
\hline Runde & Projekt & Auflagen \\
\hline & GDI Steyr & $\begin{array}{ll}- & \text { Kürzung der Tagsätze auf max. } 750 \text { EUR (Kürzung um } 3.050 \text { EUR) } \\
- & \text { Darstellung der Schnittstellen bzw. der Abgrenzung zu anderen geförderten Projekten des TIC-Steyr (RWB-Steyr, ADC Steyr). } \\
- & \text { Nachweis der Abstimmung zu den Aktivitäten, die im Rahmen des tech2b gefördert werden. } \\
- & \text { Detaillierte Darstellung des konkreten geplanten Outputs (mit Quantifizierung der angestrebten Zahl der Gründungen) bzw. der Wirkungen des Projektes } \\
& \text { zur Stärkung des regionalen Innovationspotenzials } \\
& \text { Abstimmung von Zwischenergebnissen mit dem REGplus-Projekt ISB (im Falle der Förderung von ISB) }\end{array}$ \\
\hline & bio.com.net II & $\begin{array}{ll}\text { - } & \text { Die bundesseitige Förderungsintensität ist von } 40 \% \text { auf } 30 \% \text { zu reduzieren und in einem überarbeiteten Kosten und Finanzierungsplan darzustellen (bei } \\
\text { gleich bleibenden Gesamtkosten und gleich bleibenden durchzuführenden Arbeitspaketen). } \\
\text { - } \\
\text { Aufgliederung des Kostenplanes nach Leistungsträgern und Leistungsinhalten sowie Definition nachvollziehbarer Meilensteine Darstellung der Rolle der } \\
\text { - } \\
\text { assoziierten Unternehmen. } \\
\text { - } & \text { Empfehlung: Messeauftritt und Roadshow erst nach Markenentwicklung } \\
\end{array}$ \\
\hline & $\begin{array}{l}\text { hyper metall } \\
\text { processing }\end{array}$ & $\begin{array}{ll}- & \text { Streichung der Kosten des Kunststoffclusters aus den förderbaren Gesamtkosten (bei gleich bleibenden durchzuführenden Arbeitspaketen) } \\
\text { - } & \text { Überarbeitung des Kosten- und Finanzierungsplanes } \\
- & \text { Abstimmung und Abgrenzung zum protec netplus-Projekt „virTOOL“ und zu den Initiativen des Kunststoffclusters in OÖ }\end{array}$ \\
\hline & TRANSFER & $\begin{array}{l}\text { - Einnahmen, die aus dem geförderten Projekt erzielt werden, sind getrennt darzustellen und nachweislich für regionalwirtschaftliche Projekte im Bereich } \\
\text { Know How Transfer und Qualifikation einzusetzen. } \\
\text { - } \quad \text { Projektergebnisse sind dem oö. Technologienetzwerk zur Verfügung zu stellen. }\end{array}$ \\
\hline & i-weg & $\begin{array}{ll}\text { - } & \text { Erhöhung des Eigenmittelanteils auf mind. 10\%, Datierung von Meilensteinen mit messbaren Ergebnissen je Arbeitspaket und mit klarem Fokus auf die } \\
& \text { Produktentwicklungen } \\
\text { - } & \text { Detaillierung der Sach- und Drittkosten } \\
\text { - } & \text { Darstellung der Rolle der Unternehmenspartner im Projekt. } \\
& \text { Im Rahmen der Projektdurchführung Sicherstellung der bedarfsorientierten Zugriffsmöglichkeit externer Partner auf das Zerspanungslabor }\end{array}$ \\
\hline & energycenter-net & $\begin{array}{ll}- & \text { Anpassung des ÖAR Tagsatzes an die Höchstgrenze } 750 \text { EUR und Korrektur der Kostenplanung } \\
\text { - } & \text { Bundesseitige Förderungsintensität ist von } 40 \% \text { auf } 30 \% \text { der förderbaren Kosten (auf Basis des korrigierten Kostenplanes) zu reduzieren und in einem } \\
& \text { überarbeiteten Kosten- und Finanzierungsplan darzustellen (bei gleichbleibenen Gesamtkosten und gleich bleibenden durchzuführenden Arbeitspaketen) } \\
\text { - } & \text { Absichtserklärung hinsichtlich der Einbeziehung des Ökoenergie Clusters OÖ } \\
- & \text { Darstellung der Abgrenzung / Schnittstellen zu anderen geförderten die Region betreffenden Energie-Projekten } \\
\end{array}$ \\
\hline & FH Region OÖ & $\begin{array}{ll} & \text { Meilenstein mit Stop or Go Entscheidung nach der Bedarfsanalyse und Weiterführung des Projektes nur bei Vorliegen einer gemäß Bedarfsanalyse } \\
& \text { ausreichend begründeten Nachfrage. } \\
\text { - } & \text { Reduktion der Bundesförderung auf max. } 150.000 € \text { bei gleichzeitiger Umwandlung des Erweiterungsprojekts zu einem Aufbauprojekt } \\
- & \text { Detaillierung der Investitions- und Sachkosten, Darstellung differenzierter Tagsätze und korrigierte Darstellung in den Tabellen }\end{array}$ \\
\hline
\end{tabular}

Quelle: Juryprotokolle der jeweiligen Ausschreibungsrunden, FGG 


\subsection{Trefferbilanz: Die Verteilung der Projekte und Fördermittel nach Bundesländern}

Die Durchführung des RIF 2000 Programms und damit verbunden, Errichtung und Betrieb von Impulszentren mit hoher Ausstrahlwirkung nicht nur zu den einmieteten Unternehmen hin, sondern auch in die lokale / regionale Wirtschaft, ist ein deutlicher Ausdruck der Auffassung, wonach es Politikelemente gibt, die einen hohen Grad an 'Räumlichkeit' aufweisen. Darunter sind kurze Distanzen - die berühmte halbe Stunde mit dem Auto $^{29}$-, die kommunikative Nähe, die Dominanz informeller Beziehungen gegenüber bürokratischen, d.h. regelgebundenen Verfahren, zu verstehen.

Die meisten Bundesländer haben in den vergangenen 5-10 Jahren auf diese Herausforderung mit einer Reihe von Maßnahmen reagiert ${ }^{30}$. Herausragend sind dabei Clusterinitiativen, Technologie- und Impulszentren sowie eine besondere Vorliebe für angewandte Forschungseinrichtungen ${ }^{31}$.

Der Bund, allen voran das Bundesministerium für Verkehr, Innovation und Technologie sowie in Teilen auch das Bundesministerium für Wirtschaft und Arbeit haben seit Ende der 90er Jahre zunehmend mehr Programme aufgelegt, bei denen grundsätzlich eine Ko-Finanzierung vorgesehen ist: Kplus, $\mathrm{K}_{\text {ind }}, \mathrm{K}_{\text {net }}$, FHplus ${ }^{32}$, RIF 2000 / REGplus, AplusB. Diese Programme sind, mit wenigen Ausnahmen, allem Anschein nach von hoher Attraktivität für die jeweiligen Bundesländer. Während Kplus, FHplus und AplusB mehr oder weniger an die Existenz von Universitäten bzw. Fachhochschulen gebunden sind, ist REGplus an etwas gebunden, was es in Österreich in einem im internationalen Vergleich geradezu üppigen Ausmaß gibt: Technologie- und Impulszentren. Wenn es also innerhalb dieser prominenten Bundesprogramme mit Ko-Finanzierung durch die Länder ein wirklich regionales Programm gibt, welches den geringsten Grad an infrastrukturellen Zutrittsbarrieren aufweist, dann ist es jedenfalls das RIF 2000 / REGplus Programm. Umgekehrt ist es so, dass sich ein Impulszentrum immer einrichten lässt, und sei es nur im Ausmaß eines kleineren Büros mit 34,92 $\mathrm{m}^{2}$ (namentlich in Gänserndorf).

Aus diesen Überlegungen ist es also doppelt interessant, die Verteilung der Projekte und Fördermittel über die einzelnen Bundesländer hinweg zu betrachten. Die folgenden Tabellen liefern hierzu zahlreiche Einsichten.

29 Oder, auf dem Land gerechnet: 30-40 km. Diese Einschätzung stammt von Georg Kreilinger (Techno-Z Braunau) und ist konsistent mit einschlägigen Befunden der Innovationsforschung. Seiner Erfahrung nach ist dies auch der Einzugsbereich für neue Mieter, die Reichweite seiner regionalen Betreuung und in der Folge auch die Demarkationslinie zwischen Kooperation und Kollegialität zwischen den Zentren und dem Punkt, wo man sich die Mieter gegenseitig 'abjagt'.

30 Vgl. für einen Überblick Ohler, F., Neue Wege in der Forschungs-, Technologie- und Innovationspolitik zwischen Bund und Bundesländer, im Auftrag des Rats für Forschung und Technologieentwicklung, Technopolis, Wien 2004

31 Oberösterreich, Salzburg, Kärnten und Niederösterreich haben diesen Pfad neu für sich entdeckt und eigene Landesforschungsgesellschaften eingerichtet. Die Steiermark tut dies seit jeher. Tiroler Wissenschaftler waren erfolgreich im Einwerben von Kompetenzzentren und -netzwerken und Tirol schickt sich nun an, diese stärker in die beiden 'Primär'sektoren, den Unternehmens- und den Universitätssektor, zurück zu binden. Ähnlich versucht es auch Wien, die jeweiligen Primärsektoren zu stärken.

32 Beim FHplus Programm trifft die Erfordernis der Ko-Finanzierung allerdings nur indirekt zu, u.zw. insoweit der Fachhochschulträger häufig eine Gesellschaft des Landes ist. 
Tabelle 18 gibt eine Übersicht über die Verteilung der Bundesförderung nach Bundesländern über alle drei Ausschreibungsrunden. Darin zeigen sich einige deutliche Muster. Abgesehen davon, dass sich kein einziges Zentrum aus Kärnten beteiligt hat, tun dies Zentren aus der Steiermark und aus Oberösterreich in einem umso größeren Ausmaß. Diese beiden Bundesländer lukrieren mit 39\% bzw. 29\% über zwei Drittel der gesamten Mittel, oder EUR 2.319.406 bzw. EUR 1.770.704. Tirol kommt immerhin für 14\% auf und auch das Burgenland lukriert, gemessen an seiner Größe und Wirtschaftsstruktur, immerhin 7\%, was vor allem auch dann viel ist, wenn man es mit dem industriell besser gestellten Vorarlberg vergleicht. Das einstige Vorzeigemodell im Bereich der Impulszentren, Salzburg, bricht ein und lukriert lediglich 4\% der Fördermittel des Bundes. Niederösterreich, ein weiterer Pionier im Bereich der Impulszentren, wirbt überhaupt nur $2 \%$ der Bundesförderung ein. Wien und Kärnten gehen völlig leer aus.

Tabelle 18 Verteilung der REGplus Bundesförderung nach Bundesländern

\begin{tabular}{|l|r|r|r|}
\hline & \multicolumn{1}{|c|}{ EUR } & \% & \% kum \\
\hline Steiermark & 2.319 .406 & $39 \%$ & $39 \%$ \\
\hline Oberösterreich & 1.770 .584 & $29 \%$ & $68 \%$ \\
\hline Tirol & 836.138 & $14 \%$ & $82 \%$ \\
\hline Burgenland & 415.791 & $7 \%$ & $89 \%$ \\
\hline Vorarlberg & 305.645 & $5 \%$ & $94 \%$ \\
\hline Salzburg & 239.035 & $4 \%$ & $98 \%$ \\
\hline Niederösterreich & 125.237 & $2 \%$ & $100 \%$ \\
\hline Wien & & $0 \%$ & $100 \%$ \\
\hline Kärnten & & $0 \%$ & $100 \%$ \\
\hline Gesamt & $\mathbf{6 . 0 1 1 . 8 3 6}$ & $\mathbf{1 0 0} \%$ & $\mathbf{1 0 0 \%}$ \\
\hline
\end{tabular}

Gibt es für diese ungleiche Verteilung systematische Gründe? Wie ist das Verhältnis von Versuchen zu Treffern? Gibt es bei einzelnen Bundesländern eine übergeordnete Politik und entsprechende Koordinationsbemühungen, die bei anderen fehlen? Warum brechen insbesondere Salzburg und Niederösterreich ein, zwei Länder, die immerhin zu den Pionieren in der Errichtung von Impulszentren in Österreich gehören ${ }^{33}$.

Tabelle 19 gibt eine erste Antwort. Sie stellt dar, (i) wie hoch die Projektkosten der eingereichten Projekte in den jeweiligen Ausschreibungsrunden sind, (ii) die Höhe der Projektkosten der tatsächlich genehmigten Projekte, (iii) das Volumen der ins jeweilige Bundesland geflossenen Bundesförderung, (iv) wie viele Projekte von den jeweiligen Bundesländern in den jeweiligen Ausschreibungsrunden eingereicht wurden und wie viele schließlich (v) davon erfolgreich waren. Diese Tabelle verlangt nach einer Wanderung durch die einzelnen Bundesländer. Wir wollen dies im Folgenden tun und die wichtigsten Beobachtungen anführen.

33 Das RIZ Gründerzentrum Wr. Neustadt und das Techno-Z Salzburg wurden 1988 gegründet. Damals gab es lediglich das um zwei Jahre ältere Technologie- und Innovationszentrum Graz (vgl. Tabelle 26). 
Tabelle 19 Projektkosten, Fördermittel und Trefferbilanz nach Bundesländer und Ausschreibungsrunden

\begin{tabular}{|c|c|c|c|c|c|}
\hline & & 1. Runde & 2. Runde & 3. Runde & Alle Runden \\
\hline \multirow[t]{6}{*}{ Burgenland } & Gesamtprojektkosten beantragt [EUR] & 1.079 .965 & 775.714 & 621.600 & 2.477 .279 \\
\hline & \begin{tabular}{|l|} 
beantragte Bundesförderung* [EUR] \\
\end{tabular} & 300.000 & 310.286 & & 610.286 \\
\hline & gewährte Bundesförderung [EUR] & 240.000 & 175.791 & & 415.791 \\
\hline & eingereichte Projekte & 2 & 1 & 2 & 5 \\
\hline & geförderte Projekte & 1 & 1 & & 2 \\
\hline & Trefferquote & $50 \%$ & $100 \%$ & $0 \%$ & $40 \%$ \\
\hline \multirow[t]{6}{*}{ Niederösterreich } & Gesamtprojektkosten beantragt [EUR] & 380.079 & 2.731 .150 & 739.245 & 3.850 .474 \\
\hline & beantragte Bundesförderung* [EUR] & & 189.152 & & 189.152 \\
\hline & \begin{tabular}{|l|} 
gewährte Bundesförderung [EUR] \\
\end{tabular} & & 125.237 & & 125.237 \\
\hline & eingereichte Projekte & 2 & 5 & 2 & 9 \\
\hline & geförderte Projekte & & 1 & & 1 \\
\hline & Trefferquote & $0 \%$ & $20 \%$ & $0 \%$ & $11 \%$ \\
\hline \multirow[t]{6}{*}{ Oberösterreich } & Gesamtprojektkosten beantragt [EUR] & 1.296 .471 & 4.658 .534 & 3.299 .870 & 9.254 .875 \\
\hline & beantragte Bundesförderung* [EUR] & 361.324 & 1.360 .648 & 757.587 & 2.479 .559 \\
\hline & \begin{tabular}{|l|} 
gewährte Bundesförderung [EUR] \\
\end{tabular} & 361.324 & 760.133 & 649.127 & 1.770 .584 \\
\hline & eingereichte Projekte & 5 & 11 & 10 & 26 \\
\hline & $\begin{array}{l}\text { geförderte Projekte } \\
\end{array}$ & 3 & 7 & 6 & 16 \\
\hline & \begin{tabular}{|l|} 
Trefferquote \\
\end{tabular} & $60 \%$ & $64 \%$ & $60 \%$ & $62 \%$ \\
\hline \multirow[t]{6}{*}{ Salzburg } & Gesamtprojektkosten beantragt [EUR] & 1.501 .594 & 71.905 & 360.000 & 1.933 .499 \\
\hline & beantragte Bundesförderung* [EUR] & 297.174 & & & 297.174 \\
\hline & \begin{tabular}{|l} 
gewährte Bundesförderung [EUR] \\
\end{tabular} & 239.035 & & & 239.035 \\
\hline & \begin{tabular}{|l} 
eingereichte Projekte \\
\end{tabular} & 3 & 1 & 1 & 5 \\
\hline & geförderte Projekte & 1 & & & 1 \\
\hline & Trefferquote & $33 \%$ & $0 \%$ & $0 \%$ & $20 \%$ \\
\hline \multirow[t]{6}{*}{ Steiermark } & Gesamtprojektkosten beantragt [EUR] & 1.979 .239 & 5.022 .520 & 3.593 .514 & 10.595 .273 \\
\hline & \begin{tabular}{|l|} 
beantragte Bundesförderung* [EUR] \\
\end{tabular} & 575.247 & 1.200 .000 & 822.200 & 2.597 .447 \\
\hline & gewährte Bundesförderung [EUR] & 575.247 & 921.959 & 822.200 & 2.319 .406 \\
\hline & \begin{tabular}{|l} 
eingereichte Projekte \\
\end{tabular} & 6 & 6 & 8 & 20 \\
\hline & $\begin{array}{l}\text { geförderte Projekte } \\
\end{array}$ & 4 & 3 & 4 & 11 \\
\hline & Trefferquote & $67 \%$ & $50 \%$ & $50 \%$ & $55 \%$ \\
\hline \multirow[t]{6}{*}{ Tirol } & Gesamtprojektkosten beantragt [EUR] & 691.227 & 754.548 & 1.396 .978 & 2.842 .753 \\
\hline & beantragte Bundesförderung* [EUR] & & 400.000 & 574.786 & 974.786 \\
\hline & gewährte Bundesförderung [EUR] & & 362.326 & 473.812 & 836.138 \\
\hline & eingereichte Projekte & 3 & 2 & 2 & 7 \\
\hline & geförderte Projekte & & 2 & 2 & 4 \\
\hline & Trefferquote & $0 \%$ & $100 \%$ & $100 \%$ & $57 \%$ \\
\hline \multirow[t]{6}{*}{ Vorarlberg } & Gesamtprojektkosten beantragt [EUR] & 341.436 & 948.270 & & 1.289 .706 \\
\hline & beantragte Bundesförderung* [EUR] & & 449.308 & & 449.308 \\
\hline & gewährte Bundesförderung [EUR] & & 305.645 & & 305.645 \\
\hline & \begin{tabular}{|l} 
eingereichte Projekte [EUR] \\
\end{tabular} & 1 & 1 & & 2 \\
\hline & \begin{tabular}{|l} 
geförderte Projekte \\
\end{tabular} & & 1 & & 1 \\
\hline & Trefferquote & $0 \%$ & $100 \%$ & & $50 \%$ \\
\hline \multirow[t]{6}{*}{ Wien } & Gesamtprojektkosten beantragt [EUR] & 175.093 & 377.844 & & 552.937 \\
\hline & beantragte Bundesförderung* [EUR] & & & & \\
\hline & \begin{tabular}{|l|} 
gewährte Bundesförderung [EUR] \\
\end{tabular} & & & & \\
\hline & \begin{tabular}{|l} 
eingereichte Projekte \\
\end{tabular} & 1 & 1 & & 2 \\
\hline & geförderte Projekte & & & & \\
\hline & \begin{tabular}{|l|} 
Trefferquote \\
\end{tabular} & $0 \%$ & $0 \%$ & & $0 \%$ \\
\hline \multirow[t]{7}{*}{ Gesamt } & Gesamtprojektkosten beantragt [EUR] & 7.445 .103 & 15.340 .485 & 10.011 .207 & 32.796 .796 \\
\hline & beantragte Bundesförderung* [EUR] & 1.533 .745 & 3.909 .393 & 2.154 .573 & 7.597 .712 \\
\hline & gewährte Bundesförderung [EUR] & 1.415 .606 & 2.651 .091 & 1.945 .139 & 6.011 .836 \\
\hline & \begin{tabular}{|l} 
eingereichte Projekte \\
\end{tabular} & 23 & 28 & 25 & 76 \\
\hline & geförderte Projekte & 9 & 15 & 12 & 36 \\
\hline & Trefferquote & $39 \%$ & $54 \%$ & $48 \%$ & $47 \%$ \\
\hline & Kürzungen & $92 \%$ & $68 \%$ & $90 \%$ & $79 \%$ \\
\hline
\end{tabular}

* von jenen Projekten, die grundsätzlich als förderungswürdig anerkannt wurden

Quelle: FFG 


\section{Burgenland}

Startet mit Engagement in der ersten Runde, schließt mit einem Folgeprojekt an und erlebt in der dritten Runde zwei Fehlversuche. Die Projekte sind überdurchschnittlich groß. Für das Burgenland haben die ersten REGplus Projekte prägenden Charakter für die Governance der Innovationspolitik des Landes. Die verlorenen Projekte sollten diese Grundversorgungsfunktion weiterführen, nicht zuletzt weil im Burgenland dieses Politikfeld noch nicht so stark entwickelt ist wie in den meisten anderen Bundesländern, vor allem genießt es noch nicht die wünschenswerte Aufmerksamkeit.

\section{Kärnten}

Dieses Bundesland scheint in der Landkarte der Bewerbungen um REGplus Projekte nicht auf. Hierfür sind mehrere Faktoren verantwortlich. Zum Einen hat Kärnten, abgesehen vom Technologiepark Klagenfurt (1992), diesen Politikpfad erst relativ spät betreten. Dies war gepaart mit einer relativ hohen Fluktuation sowohl bei den auf Landesseite verantwortlichen Institutionen als auch Personen. Wie wir aus anderen Kontexten wissen, sind personelle Kontinuität und politische Stabilität nicht unwesentliche Erfolgsfaktoren. Neben der geringen Aufmerksamkeit aufgrund von Diskontinuitäten wurde in den letzten Jahren viel Aufmerksamkeit der Errichtung des Großprojekts Lakeside gewidmet.

\section{Niederösterreich}

Mit neun eingereichten und einem gewonnenen Projekt bilanziert Niederösterreich zahlreiche Fehlversuche. In der letzten Runde hat zudem die Landesregierung die Ko-Finanzierung abgelehnt, so dass einige Projektanträge, die halb fertig waren, in der Schublade verschwanden. Interessant ist in Niederösterreich, dass eine Reihe von Projekten, die durchaus dem Charakter von REGplus-Projekten entsprechen, aus eigenen Mitteln bestritten werden. Niederösterreich wäre im Prinzip gut ausgestattet, zum Einen weil es über eine große Zahl an Impulszentren verfügt (17) und zum Anderen seine Politik im Bereich Impulszentren durch die RIZ-Holding vergleichsweise stark zentralisiert ist.

\section{Oberösterreich}

Reicht von allen Bundesländern die meisten Projekte (26 aus 76) ein und gewinnt auch die meisten (16 aus 36). Über alle drei Ausschreibungsrunden konstante hohe Trefferquote (60-64\%). Die Projekte sind eher unterdurchschnittlich groß. Oberösterreich verfolgt eine zentral koordinierte Politik in Bezug auf Impulszentren. Die dominanten Koordinationsmechanismen sind (i) Eigentum (nicht unähnlich in Niederösterreich, Salzburg oder große Teile der Steiermark) und (ii) zentrale Koordination im Bereich Qualitätsmanagement, im vorliegenden Fall der jeweiligen Projektansuchen, Schnittstelle zum Land. Zentraler Akteur ist hier ohne Zweifel die Technologie Marketing Gesellschaft (TMG), die sowohl an weithin allen Zentren Eigentum hält als auch und vor allem die zentrale Koordinationsfunktion wirkungsvoll ausübt.

\section{Salzburg}

Ein Treffer in der ersten Runde. Seither vergebliches Bemühen, allerdings auf insgesamt niedrigerem Niveau (5 eingereichte Projekte). Diese schwache Bilanz steht im Einklang mit einer schwachen Bilanz bei Bemühungen aus anderen einschlägigen Ko- 
Finanzierungsprogrammen. Erst in jüngster Vergangenheit hat Salzburg ein derartiges Projekt gewonnen, namentlich ein AplusB Zentrum. Die einstige Flaggschiffrolle ist Salzburg jedenfalls deutlich abhanden gekommen. Interesse aus Salzburg gibt es indes weiterhin, was sich daran zeigt, dass in jeder Ausschreibungsrunde Anträge vorgelegen haben. Die seit zwei Jahren existierende Innovations- und Technologietransfer Gesellschaft (ITG), eine gemeinnützigen Gesellschaft des Landes Salzburg, der Wirtschaftskammer Salzburg, des Techno-Z Verbunds, der Industriellenvereinigung Salzburg, der Universität Salzburg, der FH Salzburg, der Salzburg Research und der StandortAgentur Salzburg, versucht hier, die einst führenden Rolle des Techno-Z Verbunds zurück zu gewinnen. Das AplusB Zentrum ist ein sichtbarer Ausdruck dieser Bemühungen.

\section{Steiermark}

Die Steiermark rangiert zwar hinter Oberösterreich, was die Zahl der eingereichten bzw. gewonnenen Projekte betrifft. Weil sie aber im Schnitt aber fast doppelt so groß sind, lukriert die Steiermark das meiste Geld (39\% des Gesamtbudgets). Vier der fünf größten Projekte kommen aus der Steiermark. Die Steiermark verfügt durch die landeseigene Steirische Wirtschaftsförderung (SFG) eine mächtige Organisation, die aktive Politik betreibt und zugleich einen Grad an Kontinuität im Bereich der Impulszentren aufweist. Die Inanspruchnahme von Programmen mit Ko-Finanzierungscharakter ist in der Steiermark generell stark ausgeprägt.

\section{Tirol}

Tirol hat in der ersten Ausschreibungsrunde drei Projekte eingereicht, allerdings mit vollständig negativem Ausgang. Anders in den Ausschreibungsrunde zwei und drei, wo Tirol eine hundertprozentige Ausbeute verzeichnen konnte. Dies hat nicht unwesentlich mit einem beachtlichen Anstieg an einschlägigen intermediären Organisationen in den letzten Jahren und deren Professionalisierung zu tun: Re-Orientierung der Tiroler Zukunftsstiftung, AplusB CAST, diverse Kompetenzzentren aus dem $\mathrm{K}_{\text {ind }}$ bzw. $K_{\text {net }}$ Programm ${ }^{34}$.

\section{Vorarlberg}

Zwei Einreichungen, eine erfolgreich. Auffallend ist hier, dass es sich dabei um ein großes (insgesamt das drittgrößte) Projekt eines privaten Trägers eines Technologiezentrums handelt. Generell hat Vorarlberg sich zu einer Politik entschlossen, die wesentlich auf das Konzept des Public-Private-Partnership (PPP) baut, was in den vergangenen neun Jahren zu insgesamt acht Zentren geführt hat. Privater Partner ist jeweils die von Vorarlberg aus agierende PRISMA-Holding.

\section{Wien}

Es gibt zwei, allerdings erfolglose Projekte. Das 'offizielle' Wien tut allerdings nicht mit. Gemeint sind hier die Impulszentren im Umfeld des Zentrums für Innovation und Technologie (ZIT) bzw. des WWFF. Hauptzuständig für Einreichungen wäre das ZIT gewesen. Dieses befand sich während der Laufzeit des RIF 2000 Programms gerade

34 Vgl. F. Ohler, M. Stampfer, Audit und Re-Organisationskonzept für die Beteiligungen der Tiroler Zukunftsstiftung an Forschungs- und Technologietransfereinrichtungen, Technopolis und WWTF, Juli 2005. 
im Aufbau und war zum Einen mit der baulichen Errichtung von Zentren einerseits und mit der Konzeption und Umsetzung von Förderprogrammen andererseits beschäftigt. Die Nicht-Teilnahme war jedenfalls keine Frage der Geringschätzung, sondern eine Frage geringer Aufmerksamkeit.

\section{Schlussfolgerungen}

- Knapp die Hälfte aller eingereichten Projekte (47\%) werden gefördert. Die förderungswürdigen Projekte werden überdies um 20\% gekürzt. Diese Kürzung verteilt sich auf genau die Hälfte (50\%) der geförderten Projekte. Der Anteil der abgelehnten Projekte ist für frühere Verhältnisse, in denen das so genannte Antragsprinzip vorherrschend war, ziemlich hoch ${ }^{35}$. Allerdings hat sich in den vergangenen fünf Jahren, vor allem durch die Einführung von Jurys als de facto Entscheidungsinstanz, eine Erfolgrate durchgesetzt, die von den Zielgruppen durchaus akzeptiert wird. Projekte aus dem REGplus liegen hier im Mittelfeld und es waren kaum Stimmen zu vernehmen, an dieser Praxis etwas zu verändern.

- Das Beteiligungsverhalten ist von Bundesland zu Bundesland und von Runde zu Runde verschieden. Ausnahmen bilden Oberösterreich und die Steiermark, die eine hohe Systematik in der Wahrnehmung der Aufgabe Technologie- und Impulszentren entwickelt haben. In Tirol, als weitere Ausnahme, hat sich die institutionelle Landschaft in den letzten Jahren deutlich verdichtet und professionalisiert, was sich unmittelbar in den Ergebnissen der zweiten und dritten Runde ausdrückt.

- Es gibt keine wie immer gearteten Hinweise auf eine Kontingentierung der Fördermittel zugunsten bestimmter Bundesländer oder auf Basis bestimmter Schlüssel. Der einzige Maßstab, der bei der Förderung zur Anwendung gekommen ist, ist die Qualität des Antrags. Mehr durch glückliche Umstände als durch planende Hand war es möglich, dass keine, im Prinzip als förderungswürdig erachteten Projekte aufgrund von Budgetknappheit abgelehnt werden mussten mehr oder weniger. Während nämlich in den ersten beiden Runden dieses Prinzip säuberlich durchgehalten werden konnte, mussten in der dritten Ausschreibungsrunde die drei schwächsten der im Prinzip förderungswürdige Projekte mangels Budget abgelehnt werden.

- Die hohe Ablehnungsquote, die Ungleichheit im Beteiligungsverhalten sowie, und vor allem, die Allgegenwärtigkeit von Reduktionen und Auflagen, geben einen Hinweis auf die Schwierigkeit, (i) das Programm auf die spezifischen Probleme und Möglichkeiten der Technologie- bzw. Impulszentren abzustimmen und (ii) es zu den Adressaten hin zu vermitteln. Dies ist umso merkwürdiger, als man meinen möchte, dass es sich bei Impulszentren zum Einen um eine ausgesprochen homogene Zielgruppe handelt und zum Anderen, dass diese hoch organisiert ist. Zu erwähnen sind hier - mit unterschiedlichen Funktionen und Reichweiten - der Verband der Technologiezentren Österreichs (VTÖ), der Verbund oö. Technologiezentren, in der alle oberösterreichischen Technologiezentren lose zusammen gefasst sind in Verbindung mit der Eigentümer- und Koordinationsfunktion durch die TMG, die eigentumsrechtliche Zusammenführung der meisten niederösterreichischen Zentren in der RIZ-Holding oder die hohe

35 Diese geringe Ablehnungsrate hat vielfach auch damit zu tun, dass in diesem Verfahren ungeeignete Projekte bereits im Vorfeld ausgeschieden werden können. 
Präsenz der Steirischen Wirtschaftsförderung (SFG) in der Doppelrolle als Miteigentümer von Zentren in Verbindung mit der Rolle als Förderer.

- Als wichtigste Verhaltenselemente und bestimmende Erfolgsfaktoren erachten wir zwei Dinge, die sich bei genauerem Hinsehen als verschiedene Ausprägungen desselben Verhaltens heraus stellen. Es sind dies 'Aufmerksamkeit' und 'strategische Intelligenz'. Keiner der sonstigen Faktoren prägt, über alle Bundesländer hinweg betrachtet, die Erfolgsbilanz so sehr, wie diese beiden. An zahlreichen Fällen lässt sich dies positiv nachzeichnen, aber auch und gerade an den beiden Ländern, wo der Option RIF 2000 eben zu wenig Aufmerksamkeit geschenkt wurde. Diese Bedeutung von Aufmerksamkeit und strategischer Intelligenz korrespondiert auch stark mit der Beobachtung, dass die Koinzidenz mit anderen Ereignissen zu einer reduzierten Aufmerksamkeit und Wahrnehmung führt.

- Wir kommen zu dem weit reichenden Schluss, dass unterschiedliche Länder unterschiedliche Absorptionskapazitäten für Politikoptionen mitbringen. Natürlich ist es am Ende das einzelne Impulszentrum und dessen Management, dass ein Konsortium aufstellt, den Antrag verfasst und später das Projekt umsetzt. Dennoch halten wir daran fest, dass es auch die Länder - verstanden hier als Wirtschaftsagentur bzw. Landesregierung und deren Akteure, Programme und Maßnahmen - sind, die wesentlich die Präsenz des einzelnen Zentrums bzw. Projekts bestimmen. 


\section{Der Entstehungskontext von RIF 2000 Projekten}

Die 'Erfindung' von RIF 2000 beruhte auf der Beobachtung, dass Impulszentren grosso modo zu wenig Aufmerksamkeit sowohl der Betreuung der Mieter hinsichtlich ihres Überlebens ('Brutkasten') und sodann ihres Wachstums ('Treibhaus') als auch der Unternehmen in der Region ('Regionalentwicklung') widmen. So war es klar, dass die Projekte aus dem RIF 2000 Programm primär dazu beitragen sollten, (i) die Kompetenz des Zentrumsmanagements zu erhöhen, diesen Aufgaben nachzukommen und (ii) diese auch wirksam werden zu lassen. Gab es Anreize genug, um diese Transformationsschritte zu tun? Wurden diese Schritte systematisch verfolgt oder 'ergaben' sich die Projekte aus dem laufenden Geschehen? Wie kontingent ist das Zustandekommen der Projekte, vor allem in Bezug auf Personen und institutionelle Faktoren? Wir können in der Tat mehrere Muster und (begünstigende) Faktoren identifizieren. Wir beschreiben zunächst diese Muster und Faktoren und ziehen im Anschluss daran Schlüsse (Kapitel 5.1). Im darauf folgenden Kapitel 5.2 beleuchten wir die inhaltliche Orientierung der einzelnen Projekte, um zu sehen, ob diese primär nach innen, also an die Mieter gerichtet sind oder überwiegend nach außen, also an die Unternehmen und Institutionen in der Region. Wie sich zeigen wird, gibt es auch noch eine dritte Kategorie, nämlich die Errichtung von materieller (Labors) oder immaterieller Infrastruktur (Schulungen, Informationspools). In Kapitel 5.3 gehen wir der spannenden Frage nach, inwieweit die Zentren nicht nur eines oder mehrere Projekte aus dem RIF 2000 abwickeln, sondern vielmehr über ein Portfolio von Projekten verfügen. Wir haben Anlass, letzteres zu vermuten und zeigen in diesem Kapitel auf, inwieweit dies der Fall ist und ziehen Schlüsse daraus.

\subsection{Wie kommen RIF 2000 / REGplus Projekte zustande?}

Wir haben im vorstehenden Kapitel feststellen können, dass die einzelnen Länder eine deutlich unterschiedliche Performance in der Inanspruchnahme des REGplus Programms aufweisen. Wir haben auch Argumente gefunden, die die Prägung durch das Bundesland, dessen Politik und dessen institutioneller Ausstattung plausibel machen. In diesem Kapitel wollen wir der Frage nachgehen, aus welchen Motiven und in welchen Kontexten die Projekte entstehen und dann versuchen, ein paar Schlüsse daraus zu ziehen. Tabelle 20 gibt einen ersten Überblick.

Tabelle 20 Motive und Umstände für das Zustandekommen von REGplus Projekten

\begin{tabular}{|c|c|}
\hline & Beschreibung [in Form von Zitaten, und vielfach retrospektiv] \\
\hline \multirow[t]{3}{*}{$\begin{array}{l}\text { bessere Nutzung } \\
\text { vorhandener } \\
\text { Ressourcen }\end{array}$} & $\begin{array}{l}\text { Mit der HTL / FHS enger zusammenarbeiten und strategischer Partner werden: } \\
\text { Lehrer gründen Firmen, wirken als Berater, Aufbau einer gemeinsame } \\
\text { Infrastruktur (z.B. Labor) mit der HTL }\end{array}$ \\
\hline & $\begin{array}{l}\text { FHS bietet berufsbegleitend Kurse / Module an, dadurch Weiterbildung + } \\
\text { Verbleib in der Region, Umbau der Lehrpläne als Auslöser }\end{array}$ \\
\hline & Firmen in der Region behalten, schauen, dass die Mitarbeiter nicht abwandern \\
\hline \multirow[t]{3}{*}{ Vernetzen } & $\begin{array}{l}\text { Anbahnen von Kooperationen zwischen den Firmen im Zentrum und / oder mit } \\
\text { solchen außerhalb }{ }^{36}\end{array}$ \\
\hline & Informationsdrehscheibe für Firmen im Zentrum und in der Region sein \\
\hline & losere Kontakte zusammenführen und festigen \\
\hline
\end{tabular}

36 Das machen typischerweise Clusterinitiativen, aber jeweils nur in ausgewählten Themenbereichen. 


\begin{tabular}{|c|c|}
\hline & Beschreibung [in Form von Zitaten, und vielfach retrospektiv] \\
\hline \multirow[t]{6}{*}{ Chancen ergreifen } & Es liegt immer irgend etwas in der Luft! \\
\hline & $\begin{array}{l}\text { Ich gehe immer mit irgendwelchen Ideen schwanger. Wenn sich eine } \\
\text { Gelegenheit wie REGplus ergibt, ergreife ich sie, wenn nicht, suche ich andere. }\end{array}$ \\
\hline & $\begin{array}{l}\text { Die Manager der Zentren treffen sich all } 3 \text { Monate und besprechen gemeinsame } \\
\text { Interessen und Projekte bzw. Erfahrungen. Da kann man dann schnell } \\
\text { zuschlagen. }\end{array}$ \\
\hline & $\begin{array}{l}\text { Man ist der Region unterwegs, und weiß, was gebraucht wird. Wir haben einen } \\
\text { guten Draht zu den Firmen und können innerhalb von } 2 \text { Monaten immer } \\
\text { irgendwas auf die Beine stellen. }\end{array}$ \\
\hline & Als Zentrumsmanager ist man immer im Stand-by-Modus. \\
\hline & $\begin{array}{l}\text { Wir können nicht alles abdecken, daher müssen wir uns profilieren und } \\
\text { zusammenarbeiten. REGplus hat uns dabei geholfen. }\end{array}$ \\
\hline \multirow[t]{2}{*}{$\begin{array}{l}\text { Erstrecken des } \\
\text { Planungshorizonts }\end{array}$} & $\begin{array}{l}\text { Mit einem Projekt in der Hand, kann ich die Firmen locken, etwas jenseits ihres } \\
\text { Tagesgeschäftes zu unternehmen. }\end{array}$ \\
\hline & $\begin{array}{l}\text { Wir können dann ein paar Dinge über eine längere Zeit ausprobieren, die } \\
\text { ansonsten auf der Strecke bleiben. Auch das ist ein Anreiz, mit dem ich } \\
\text { Hausieren gehen kann. }\end{array}$ \\
\hline \multirow{7}{*}{$\begin{array}{l}\text { Erfüllung der } \\
\text { Mission des } \\
\text { Zentrums / der } \\
\text { Politik des Landes }\end{array}$} & $\begin{array}{l}\text { In unserem Zentrum gibt bei uns (thematische) Schwerpunkte und wir suchen } \\
\text { nach Möglichkeiten, diese umzusetzen. REGplus ist so eine Chance. }\end{array}$ \\
\hline & $\begin{array}{l}\text { Manchmal gibt es keine Spezialisierung in der Region, dann muss ich über die } \\
\text { Grenze gehen und eine Brücke schlagen. }\end{array}$ \\
\hline & Wir machen das immer top-down, weil wir eine Aufgabe zu erfüllen haben. \\
\hline & $\begin{array}{l}\text { Ich hatte eine Zentrum übernommen, wusste aber nicht viel über das weitere } \\
\text { Umfeld. Also habe ich eine Studie gemacht und dabei alle Akteure einbezogen. } \\
\text { REGplus kam mir dabei gelegen. }\end{array}$ \\
\hline & Wir haben ein Thema, wollen dies vorantreiben und suchen nach Förderung. \\
\hline & Wir wollten unser Kerngeschäft systematisieren und verstärken. \\
\hline & $\begin{array}{l}\text { Die Idee kam ursprünglich vom Land, da sollte es im Rahmen des Strategischen } \\
\text { Konzepts einen Business Inkubator geben, möglichst aus dem privaten Sektor. } \\
\text { Als dann REGplus sich anbot, haben wir uns beworben. Ohne die Förderung } \\
\text { hätten wir nicht gestartet. }\end{array}$ \\
\hline \multirow{2}{*}{$\begin{array}{l}\text { Ausschreibung als } \\
\text { Trigger und } \\
\text { Fokussierungs- } \\
\text { mechanismus }\end{array}$} & $\begin{array}{l}\text { Wir stehen voll hinter dem Ausschreibungsprinzip, weil das unsere } \\
\text { Aufmerksamkeit schärft. Themen gibt es immer - ich habe immer ein paar in der } \\
\text { Pipeline. Mit der Ausschreibung müssen wir dann konkret werden. }\end{array}$ \\
\hline & $\begin{array}{l}\text { Das erste REGplus Projekt war ein Hammer. Ich war im Zuge der Vorbereitung } \\
\text { bei } 50 \text { Unternehmen. Als dann das Projekt tatsächlich kam, hatte ich deutlich an } \\
\text { Glaubwürdigkeit gewonnen. So etwas trägt dann zur Mythenbildung bei, von } \\
\text { dem man wieder ein paar Jahre leben kann. }\end{array}$ \\
\hline \multirow{3}{*}{$\begin{array}{l}\text { Leitfigur / } \\
\text { Katalysator / } \\
\text { Koordinator }\end{array}$} & $\begin{array}{l}\text { Aber man braucht dann immer eine Person, die das in die Hand nimmt, und } \\
\text { durchträgt. Manchmal muss man auch den Zufall heraufbeschwören. }\end{array}$ \\
\hline & $\begin{array}{l}\text { Es ist immer auch wichtig, zum Land einen guten Draht zu haben und die Dinge } \\
\text { abzustimmen, sonst empfinden die das als Einmischung. }\end{array}$ \\
\hline & $\begin{array}{l}\text { Eine Inkubationszeit von 3-4 Monaten ist schon sehr wichtig. Dann geht es in 2- } \\
3 \text { Wochen. }\end{array}$ \\
\hline \multirow[t]{3}{*}{$\begin{array}{l}\text { Deckungsbeiträge } \\
\text { erwirtschaften }\end{array}$} & $\begin{array}{l}\text { Wir müssen in unseren Zentren schauen, dass wir leben können. Die Mieterlöse } \\
\text { reichen nicht aus. Daher muss ich mich nach anderen Einkommensmöglichkeiten } \\
\text { umsehen. REGplus hat eine satte Förderquote. }\end{array}$ \\
\hline & $\begin{array}{l}\text { Die Zentrumsmanager sind einfach 'arme Schweine', die, wenn man's genau } \\
\text { nimmt, gar nicht leben dürften. Daher sind sie auf Förderungen wie REGplus } \\
\text { angewiesen. Langfristig zerreißen sie sich alle. }\end{array}$ \\
\hline & $\begin{array}{l}\text { Wir müssen eigene verkaufbare Dienstleistungen anbieten und versuchen sie u.a. } \\
\text { mit REGplus zu entwickeln. }\end{array}$ \\
\hline
\end{tabular}

Quelle: Interviews

Diese Liste ließe sich noch beliebig fortsetzen und verfeinern. Gleichwohl reicht sie aus, um auf folgende kritischen Faktoren aufmerksam zu machen, die für das Zustandekommen von (erfolgreichen) REGplus-Projekten maßgeblich sind. 
- Guter 'Draht' zu den Unternehmen drinnen wie draußen. Das Aufsetzen eines REGplus Projekts kann Türen öffnen, das Gewinnen des Projekts sie dauerhaft offen halten. Testfall: Bekommt der Zentrumsmanager einen Termin beim 'Chef' und wie schnell kriegt er ihn ${ }^{37}$ ?

- Triggerfunktion und Inkubationszeit sind wichtig, um das Projekt bei den Partnern gut zu verankern. Die tatsächlich gewählte Vorlaufzeit von 3-4 Monaten wird als optimal empfunden.

- Zentrumsmanager müssen immer in einem erhöhten Anregungszustand sein und mehrere Ideen gleichzeitig verfolgen. Umsetzungsgelegenheiten müssen gesucht werden und finden sich auch. REGplus ist eine solche. Die meisten Zentren haben mehrere Projekte laufen, manche bis zu zwei Dutzend ${ }^{38}$.

- Missionen, meist als thematische Ausrichtung verstanden, sollten nicht zu hoch bewertet werden. Eine erhöhte Aufmerksamkeit gegenüber den Zeitläuften (verändertes Umfeld, neue Möglichkeiten, neue Konstellationen, manchmal auch neue Personen) dürfte wichtiger sein für das Zustandekommen von Deals als eine strikte Einhaltung einer Mission.

- Enger Kontakt zur Entwicklungsagentur des Landes bzw. zum Land ist aus mehreren Gründen wesentlich. Die Wichtigsten sind Vertrauensaufbau und Teilhabe am Informationsfluss und Strategieprozess.

- Zentrumsmanager zeichnen sich dadurch aus, dass sie über ein gutes Verständnis des regionalen und überregionalen Systems verfügen und die Möglichkeit des Zugangs zu diesem System besitzen.

Wenn man berücksichtigt, dass diese Befunde aus 2005 stammen und auf die Periode 2001-2004 blicken, dann kann man den Schluss ziehen, dass sich die Zentrumsmanager vollständig im systemischen Modus befinden. Wenn dies ein Ziel war, und es war ein Ziel, so ist es, zumindest auf der Ebene der Selbstwahrnehmung und Selbstdefinition, vollständig erreicht ${ }^{39}$.

37 Dies hat auch damit zu tun, dass vielerorts bereits eine Sättigung mit Unterstützungsdienstleistungen und Förderungen zu beobachten ist. Dazu kommt, dass in der Folge eine kleinere Gruppe und bereitwilligen Unternehmen immer wieder angesprochen werden. Als Folge davon gibt es mitunter einen Wettbewerb um die Gunst von Förderadressaten. Auf diesen Punkt kommen wir weiter unten in Kapitel 5.3 noch zu sprechen.

39 Wir kommen auf diese Beobachtung, nunmehr mit einem höheren Grad an Testbarkeit, in Kapitel 5.2 noch einmal zurück. Dort geht es um die inhaltliche Orientierung der. 
Abbildung 2 Größenverteilung der Impulszentren [Zahl der Mieter]

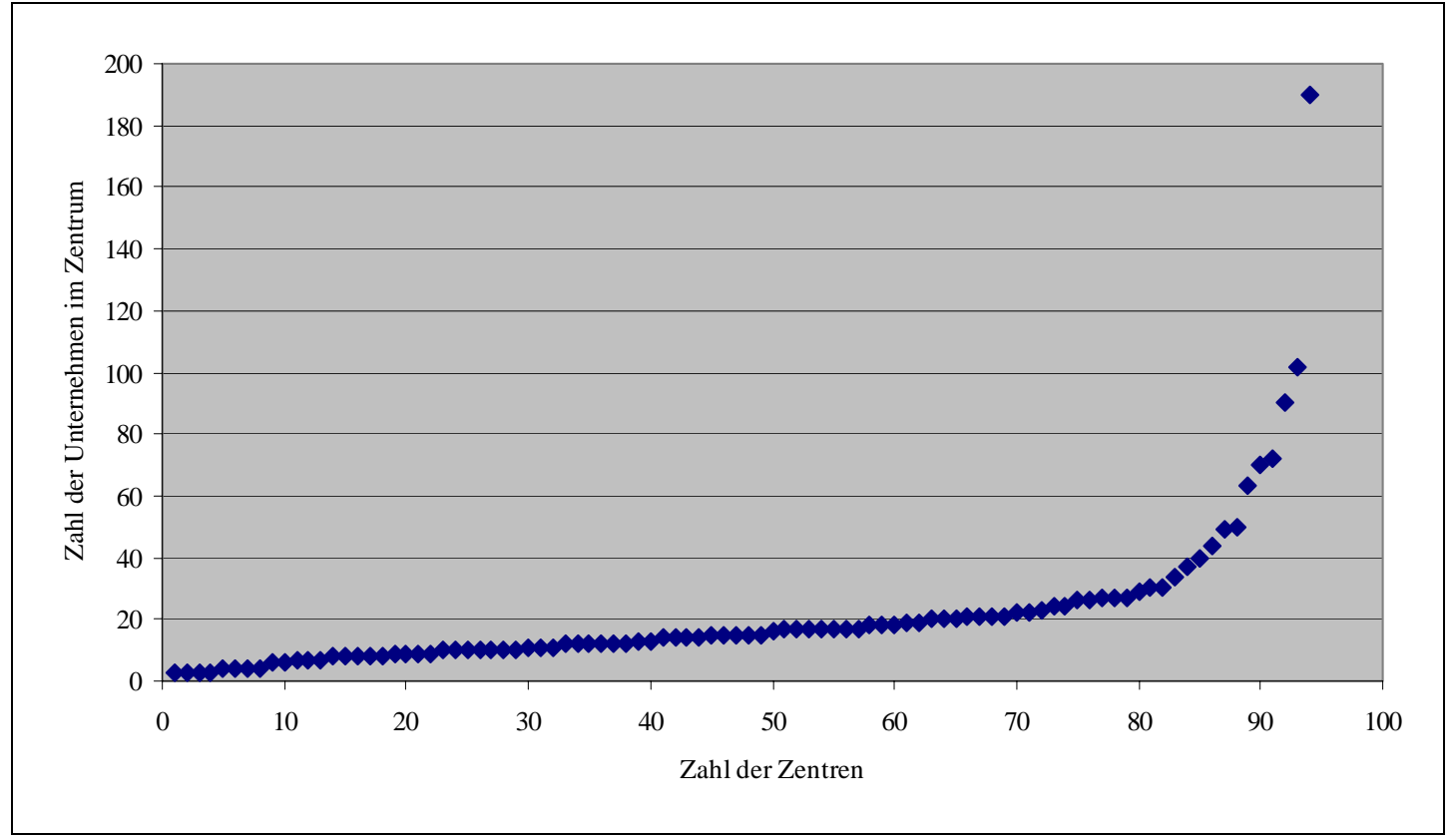

Quelle: Verband der Technologiezentren Österreichs, www.vto.at

Abbildung 3 Größenverteilung der Impulszentren [vermietbare Fläche]

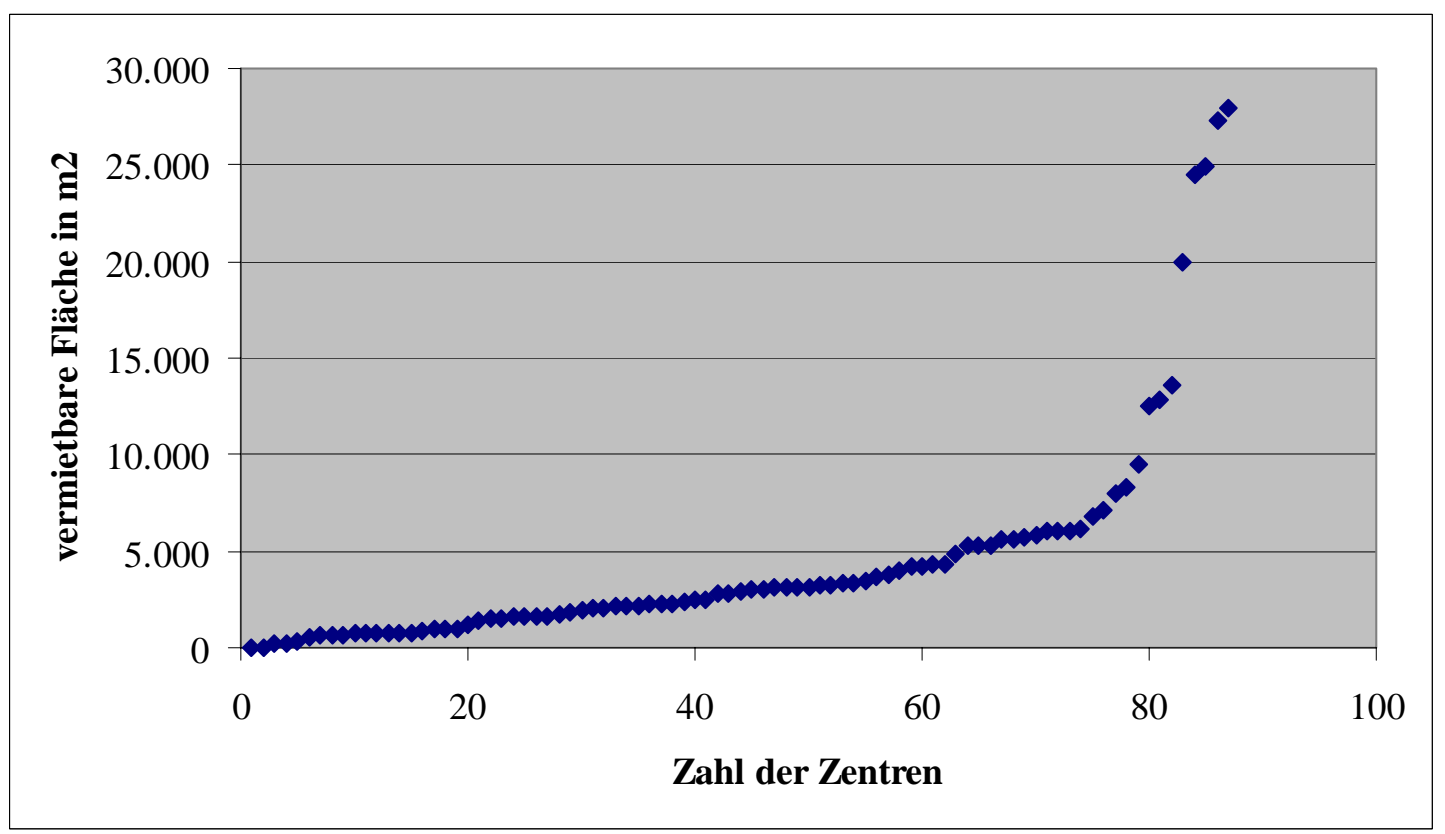

Quelle: Verband der Technologiezentren Österreichs, www.vto.at

NB: Nicht in der Abbildung eingetragen sind noch zwei weitere Zentren mit 55.000 und $120.000 \mathrm{~m}^{2}$.

Wir können das Kapitel über die Motive und Umstände für das Zustandekommen von REGplus Projekten nicht abschließen, ohne nicht auf eine sehr ernüchternde Beobachtung hinzuweisen. Es ist dies die Anerkennung der Notwendigkeit, Einkommen und damit Deckungsbeiträge zu erzielen. Hier liegt in der Regel folgendes Problem vor, das bei genauerer Betrachtung aus einer Kaskade von Problemen besteht. 
- Das Zentrum wird in der Regel von einem hauptamtlichen Manager mit einem kleinen, in einzelnen Fällen auch größeren Team geführt. In einzelnen Fällen führt der hauptamtliche Manager mehrere Zentren. Es gibt schließlich auch Fälle, wo Bürgermeister oder Amtsleiter ('Gemeindesekretär') dieser Aufgabe nachkommen. Allerdings ist dies die Ausnahme.

- Das Zentrum ist in der Regel klein, was zur Folge hat, dass ein entsprechender Managementüberbau rasch die Kosten in die Höhe treibt. Abbildung 2 gibt eine Übersicht, aus der deutlich hervorgeht, dass über zwei Drittel der Impulszentren nicht mehr als 20 Mieter beherbergen. Abbildung 3 zeigt, dass zwei Drittel der Zentren weniger als $5.000 \mathrm{~m}^{2}$ und ein Viertel bis zu $10.000 \mathrm{~m}^{2}$ zur Vermietung anbietet.

- Die Gesellschafter der Zentren rekrutieren sich zu nicht unerheblichen Teilen aus dem öffentlichen Sektor ${ }^{40}$, in einigen Fällen kommen PPP-Modelle zur Anwendung, selten sind es rein private Betreiber ${ }^{41}$.

- Hier tut sich eine Spannung auf, die am Besten stilisiert so beschrieben werden kann: Während des Jahres sind die Gesellschafter primär an regionalwirtschaftlichen Effekten interessiert, zweimal im Jahr verwandeln sie sich aber, wenn es um Budgets und Bilanzen geht, in Krämerseelen, bei denen die vermietete Fläche und das EGT im Vordergrund stehen ${ }^{42}$. Selten wird honoriert, wenn Leerstände durch Auszug eines Mieters aufgrund von Wachstum bedingt sind ${ }^{43}$.

- Selbst Versuche, Unterstützungsvereine zu bilden, führen in erfolgreichen Fällen dazu, einige bescheidene zusätzliche Zehntausend Euro aufzustellen. Dieser Fall setzt überdies eine spezifische institutionelle Anordnung voraus, die selten vorzufinden ist und den Nachteil hat, allerlei Sonderregelungen zu begünstigen.

- Für eine bestimmte Kombination aus Größe und Managementstruktur fällt es also schwer, einen gleichmäßigen, berechenbaren Einkommensstrom aus dem Betrieb des Zentrums zu erzielen.

- In der Praxis tun sich hier mehrere Möglichkeiten auf.

- $\quad$ Die Zentrumsmanager sind teilzeitbeschäftigt und verfügen über ein zweites berufliches Standbein. Neben einer Beschäftigung im öffentlichen Sektor sind sie typischerweise als Berater tätig und erzielen auf diese Weise ein (privates) Zusatzeinkommen.

- $\quad$ Andere, das ist die Regel, greifen nach Projekten, die von Land, Bund oder der EU allein oder, was die Regel ist, in Kooperation gefördert bzw. finanziert werden. In der Regel sind sie nicht ausfinanziert, sondern müssen von dritter Seite ko-finanziert werden.

- Einige fortgeschrittene Zentren versuchen, aus dieser Finanzierungsfalle dadurch zu entkommen, dass sie Dienstleistungen entwickeln, die sich zu Marktpreisen verkaufen lassen. Dies führt wieder zur Frage der Größe und des entsprechenden Dienstleistungsportfolios.

- Nicht selten widmen sich die Zentrumsmanager überwiegend der Durchführung der Projekte und immer öfter wird dieses Geschäft des Einwerbens von Projekten betrieben, um Deckungsbeiträge zu erzielen und negative Ergebnisse aus dem

40 Dafür gibt es einerseits eine Reihe guter Gründe und andererseits entstehen einige spezifische Vorteile für das Zentrum und sein Umfeld: Höheres Committment diverser Institutionen (Kammern, Banken, Schulen (HTL, FHS), Bürgermeister), bessere Verbindung insbesondere zum Land, bisweilen auch zum Bund, und öfter noch zur EU etc.

41 Auch der Cluster rund um die Fa. PRISMA ist nicht unwesentlich von PPP-Modellen geprägt.

42 Ein Zentrumsmanager nennt dies eine Lebenslüge.

43 Dies ist ein durchgängiges Verhaltensmuster von Impulszentren. Vgl. European Commission, Enterprise Directorate-General, Benchmarking of Business Incubators, Final Report, Centre for Strategy \& Evaluation Services, February 2002 
Mietgeschäft zu kompensieren ${ }^{44}$. In diesem Zusammenhang mutet es übrigens merkwürdig an, wenn einzelne Zentren Überschüsse aus der geförderten Projekttätigkeit erzielen und dadurch die Erlöse aus der Vermietung auffetten. Eine andere, durchaus komplementäre Verhaltensweise tritt in Form von Selbstausbeutung zutage ${ }^{45}$.

- Wir unterstellen hier ein rationales und insoweit auch opportunistisches Verhalten der Mehrzahl der Zentrumsmanager: So lange auf die oben beschriebene Weise die Kostendeckung leichter zu realisieren ist, wird es eine Entwicklung in Richtung 'regionale Entwicklung' geben. Würde privates Geld leichter zu erlangen sein, etwa durch Management Fees oder Coaching-Honorare für die Mieter im Zentrum oder gegenüber Dritten, könnte die Entwicklung durchaus in eine andere Richtung gehen. In der Tat wurde im Rahmen unserer Interviews die Förderungen aus dem REGplus Programm auch dafür akklamiert, als diese eine 'anständige' Förderintensität aufweisen würden. Wir ziehen daraus einen im doppelten Sinn nüchternden Schluss: Zentrumsmanager finden eine bestimmte Anreizsituation vor und richten sich danach aus. Die, die sie vorfinden, scheint ergiebig genug zu sein. Die gute Nachricht: Zentrumsmanager verhalten sich rational und sind daher durch Anreize steuerbar. Die schlechte Nachricht: Die Früchte der öffentlichen Förderung sind süß und hängen tief.

\subsection{Inhaltlich-strategische Orientierung der geförderten Projekte}

Im Standardmodell eines Gründer-, Innovations- oder Technologiezentrums oder Science Parks kümmert sich das Managementteam um die Akquisition geeigneter Mieter und um deren Wohlergehen. Ziel dieses Bemühens ist (i) höhere Gründungsrate, (ii) höhere Überlebensrate, (iii) rascheres Wachstum. In den Zentren, die sich am REGplus Programm beteiligt bzw. eine RIFupgrade Förderung erhalten haben, herrscht dagegen eine signifikant andere Orientierung vor. Wesentlicher Teil der Aufmerksamkeit wird Projekten gewidmet, die den Charakter von Regionalentwicklung haben bzw. dem Aufbau von Infrastrukturen dienen. Dieser Behauptung wollen wir im Folgenden nachgehen. Dazu unterscheiden wir folgende drei Kategorien von Dienstleistungen:

- (immaterielle) Dienstleistungen, die primär nach innen, also an die Mieter gerichtet sind

- (immaterielle) Dienstleistungen, die primär nach außen, also an Unternehmen und sonstige Einrichtungen der Region gerichtet sind, insbesondere Netzwerkund Kompetenzaufbau

- Aufbau von höherwertiger materieller Infrastruktur, also Labors, Anwendungszentren etc. und darauf aufbauende Dienstleistungen

Tabelle 21 gibt dazu einen ersten, viel sagenden Eindruck, Tabelle 22 liefert die Details. Darin fällt eine extrem starke Ausrichtung auf die Erbringung von Dienst-

44 Manchmal gibt es auch gut bezahlte Aufträge (der Entwicklungsagentur des Landes), die einen gewissen Überschuss abwerfen und die, man liegt nicht ganz falsch mit der Vermutung, als versteckte Förderung zu verstehen sind.

45 Diese an der Schnittstelle von privaten zu öffentlichen Strukturen, Kulturen, Geschäftsmodellen und Anreizen angesiedelte Tätigkeit ist grundsätzlich anfällig für Selbstausbeutung, weil hier die Zielsysteme in der Regel komplexer, ja überfrachtet sind, die Anforderungen an Denken und Verhalten aus mehreren Kulturen stammen und Zielerreichung in aller Regel schwer zu überprüfen sind. 
leistungen in die Region auf, und dabei vor allem von auf Vernetzung und Netzwerkbildung gerichtete Aktivitäten, wobei natürlich zu bemerken ist, dass diese Dienstleistungen stets auch an die eingemieteten Unternehmen gerichtet sind. Von 76 (REGplus) +6 (RIFupgrade) $=82$ eingereichten Projekten zielen 51 (REGplus) + 2 (RIFupgrade) $=53$ Projekte (65\%), also fast zwei Drittel, auf Zielgruppen ab, die sich überwiegend außerhalb der Zentren befinden. Bemerkenswert ist ferner die nicht unerhebliche Bedeutung des Aufbaus von Infrastruktur. Immerhin wurden hier 23 (REGplus) + 3 (RIFupgrade) = 26 Projekte (34\%), also ein weiteres Drittel, eingereicht, die darauf abzielen, dass über die soweit aufgebaute Infrastruktur Dienstleistungen angeboten werden können. Auch sie zielen in der Regel auf externe Zielgruppen ab. Es gibt lediglich 2 (REGplus) + 1 (RIFupgrade) = 3 eingereichte Projekte, welche wesentlich nach innen gerichtet sind. Bei einem der beiden geförderten handelt es sich, wenig überraschend, auch um das Projekt eines Inkubators in privatem Eigentum.

Tabelle 21 Orientierung der eingereichten / geförderten REGplus / RIFupgrade Projekte: Überblick

\begin{tabular}{|l|r|r|r|r|r|r|r|r|}
\hline \multicolumn{1}{|c|}{ Runde } & \multicolumn{2}{c|}{ nach innen } & \multicolumn{2}{c|}{ nach außen } & \multicolumn{2}{c|}{ Infrastruktur } & \multicolumn{2}{c|}{ gesamt } \\
\hline & $\begin{array}{l}\text { einge- } \\
\text { reicht }\end{array}$ & gefördert & $\begin{array}{l}\text { einge- } \\
\text { reicht }\end{array}$ & gefördert & $\begin{array}{l}\text { einge- } \\
\text { reicht }\end{array}$ & gefördert & $\begin{array}{l}\text { einge- } \\
\text { reicht }\end{array}$ & gefördert \\
\hline 1. Runde & 0 & 0 & 15 & 5 & 8 & 4 & 23 & 9 \\
\hline 2. Runde & 1 & 1 & 19 & 10 & 8 & 4 & 28 & 15 \\
\hline 3. Runde & 1 & 0 & 17 & 8 & 7 & 4 & 25 & 12 \\
\hline Summe & 2 & 1 & 51 & 23 & 23 & 12 & 76 & 36 \\
\hline RIFupgrade & 1 & 1 & 2 & 2 & 3 & 3 & 6 & 6 \\
\hline Gesamt & $\mathbf{3}$ & $\mathbf{2}$ & $\mathbf{5 3}$ & $\mathbf{2 5}$ & $\mathbf{2 6}$ & $\mathbf{1 5}$ & $\mathbf{8 2}$ & $\mathbf{4 2}$ \\
\hline
\end{tabular}

Quelle: FFG

\section{Schlussfolgerungen}

- Wir können aus diesen Fakten einige, durchaus weit reichende Schlüsse ziehen. Das Pfefferkorn in diesen Schlussfolgerungen ist der besondere Status des einzigen privaten Inkubators, der das einzige REGplus Projekt gewonnen hat, in dem nach innen gerichtete Dienstleistungen entwickelt und angeboten werden sollen. ${ }^{46}$

- Wechseln wir zu den beiden anderen Orientierungen, nämlich der Erbringung von Leistungen nach außen bzw. externe Vernetzung sowie Aufbau von Infrastruktur, so zeigt sich, dass sich die Verteilung über die eingereichten Projekte auf die Gruppe der geförderten Projekte überträgt. Diese beiden Orientierungen stellen also keinen Vor- oder Nachteil in der Bewerbung dar.

- Erinnern wir uns: Die Ausgangssituation bestand Ende der 90er Jahre darin, dass Zentren zu sehr unter dem Gesichtspunkt einer möglichst hoch auszulastenden Immobilie geführt wurden und dabei der Entwicklungsgesichtspunkt, allen voran der eingemieteten Unternehmen, zu kurz kam. Als Folge davon wurde ein Förderprogramm konzipiert, das helfen sollte, eine breitere Perspektive zu entwickeln. Zielgruppe waren sowohl die Mieter als auch die Unternehmen und sonstigen einschlägigen Organisationen der Region und Ziel war es, die

46 Es gibt im Grunde noch ein zweites Projekt, das insofern nach innen gerichtet ist, als es auf Qualitätssicherung und gemeinsame Standards der oberösterreichischen Zentren, also auf die Meta-Ebene abzielt. 
Entwicklungsdynamik sowohl unter den Mietern als auch in der regionalen Wirtschaft zu unterstützen. Tragender Akteur, der diese Entwicklungsdynamik entfachen sollte, war das Management der Impulszentren. Dieses sollte anhand von Projekten lernen, 'einen besseren Job zu machen'. Nahe liegender Weise sollten bevorzugt solche Projekte gefördert werden, die sowohl den Lernaspekt in Bezug auf das Zentrumsmanagement als auch in Bezug auf die Region zu fördern imstande waren.

- Inzwischen sind fünf Jahre und drei Ausschreibungsrunden durchs Land gezogen und wir finden eine Situation vor, die sich völlig gewandelt hat. Nur mehr ein einziges der 36 (REGplus) + 6 (RIFupgrade) $=42$ geförderten Projekte ist im engeren Sinn darauf gerichtet, eine Entwicklungsdynamik unter den Mietern zu entfachen. Dagegen sind 23 (REGplus) + 2 (RIFupgrade) der 36 + 6 Projekte (60\%) nach außen gerichtet und suchen vor allem in der Vernetzung, bisweilen mit anderen Zentren, ihren Beitrag zur regionalen Entwicklung zu leisten. In dieser Hinsicht ist es zu einer Umkehrung der Verhältnisse gekommen, und, wie wir Anlass haben, zu vermuten, in Teilen auch zu einer 'Flucht in die Region'.

- Zu dieser kritischen Bemerkung geben uns einige Indizien Anlass, die im Folgenden kurz angeführt seien: (i) Zahlreiche Zentrumsmanager weisen im Gespräch ausdrücklich darauf hin, dass sie aus den Überschüssen ihrer Mieterlöse ihre eigenen (Grenz)Kosten nicht abdecken können und sich daher genötigt sehen, Projekte einzuwerben. Dies hat unter anderem, (ii), damit zu tun, dass die Zentren oftmals zu klein sind und eine entsprechende Betreuungskapazität nicht rechtfertigen. Dies wiederum ist (iii) darauf zurückzuführen, dass zahlreiche Zentren aus einer (regional- bzw. lokal)politischen Motivation heraus entstanden sind. Die Manager dieser Zentren haben sich, und das ist vielfach ihre explizit vorgetragene Selbstdefinition, vom Immobilienmanager zum Regionalmanager ${ }^{47}$ verwandelt. Diesen Bewusstseins- und Verhaltenswandel herbeizuführen war ein wesentliches Ziel des Programms. Seine Erreichung ist abermals bestätigt, nunmehr unter Heranziehung der inhaltlich-strategischen Orientierung der eingereichten bzw. geförderten Projekte.

47 Der Begriff 'Regionalmanager' ist hier generisch gebraucht und ist nicht zu verwechseln mit den Mitarbeitern der Förderschiene 'Regionalmanagement' (vgl. www.rm-austria.at) 
Tabelle 22 Orientierung der eingereichten / geförderten Projekte: detailliert

\begin{tabular}{|c|c|c|c|c|}
\hline Kurzbezeichnung & Beschreibung & $\begin{array}{c}\text { nach } \\
\text { innen }\end{array}$ & $\begin{array}{c}\text { nach } \\
\text { außen }\end{array}$ & $\begin{array}{c}\text { Infra- } \\
\text { struktur }\end{array}$ \\
\hline \multicolumn{5}{|l|}{ 1. Runde } \\
\hline LUHIZ & $\begin{array}{l}\text { Lungauer Holzprodukt-Impulszentrum. Ausarbeitung und Umsetzung eines gemeinsamen Holz- } \\
\text { Produktprogramms }\end{array}$ & & & $\mathrm{x}$ \\
\hline CNC-Inet & $\begin{array}{l}\text { CNC-Maschinen - Innviertel Technologie Cluster für Holz, Kunststoff und Metall Bereiche. Maschinen- } \\
\text { Sharing und Nutzungskonzept + Schulungen + gemeinsames Marketing }\end{array}$ & & & $\mathrm{x}$ \\
\hline IT-LAB & Informationstechnologien Transfer- und Applikationslabor, Kow how - IT; Technologietransfer etc. & & & $\mathrm{x}$ \\
\hline WIP West & gemeinsame Entwicklung und Vermarktung der drei regionalen IZ als WIP West & & $\mathrm{x}$ & \\
\hline CLINT & $\begin{array}{l}\text { Cluster- und Innovations-Netzwerk-Tirol. Aufbau von Innovationsmanagement-Kompetenz durch } \\
\text { Schulung und Beratung bei Tiroler KMUs }\end{array}$ & & $\mathrm{x}$ & \\
\hline IVRA & $\begin{array}{l}\text { Institut für virtuelle Realität und Architektur. Aufbau des Instituts für virtuelle Realität, welches in } \\
\text { Bereichen der Auftragsforschung (Ind) und Verbundforschung (EU) tätig sein soll }\end{array}$ & & & $\mathrm{x}$ \\
\hline tzs.net & $\begin{array}{l}\text { Technologiezentrum Salzkammergut Netzwerk. Kooperation zw. Groß- und Kleinbetrieben, Nutzung und } \\
\text { Know-how der technischen Dienstleister, etc. }\end{array}$ & & $\mathrm{x}$ & \\
\hline FIT-Log & $\begin{array}{l}\text { Aufbau eines virtuellen IZ mit Schwerpunkt Logistik (Beratungen, Know-how-Transfer, Datenbank, } \\
\text { Schulungen,...) }\end{array}$ & & $\mathrm{x}$ & \\
\hline NET_WORK & $\begin{array}{l}\text { Strukturierte Vernetzung und Kompetenzstärkung der regionale Akteure durch Workshops und } \\
\text { anschließende Pilotphase }\end{array}$ & & $\mathrm{x}$ & \\
\hline $\begin{array}{l}\text { Pilotprojekt } \\
\text { Regionalisierung von } \\
\text { Cluster- und Innovations- } \\
\text { management unter Einsatz } \\
\text { von IT }\end{array}$ & $\begin{array}{l}\text { Internetplattform für Information, Kommunikation und Kooperation der KMUs und Bevölkerung des } \\
\text { Wipptales, inkl. Moderation von Workshops unter Einbeziehung der Bevölkerung, Schulen, Kammern }\end{array}$ & & & $\mathrm{x}$ \\
\hline $\begin{array}{l}\text { High Tech Netzwerk New } \\
\text { Alpine Touch }\end{array}$ & & & $\mathrm{x}$ & \\
\hline CenterP / CenterPortal.at & $\begin{array}{l}\text { Erstellung einer Datenbank mit Infomaterial, die sowohl für zentreninterne als auch externe Unternehmen } \\
\text { nutzbar sein soll }\end{array}$ & & & $\mathrm{x}$ \\
\hline $\begin{array}{l}\text { Positionierung } \\
\text { Technologiezentrum S.I.G. } \\
\text { Wien }\end{array}$ & Schaffung eines Know-how-Netzwerkes, Erweiterung des DL-Angebotes, neue IT, Wissensmanagement & & $\mathrm{x}$ & \\
\hline
\end{tabular}




\begin{tabular}{|c|c|c|c|c|}
\hline Kurzbezeichnung & Beschreibung & $\begin{array}{c}\text { nach } \\
\text { innen }\end{array}$ & $\begin{array}{c}\text { nach } \\
\text { außen }\end{array}$ & $\begin{array}{c}\text { Infra- } \\
\text { struktur }\end{array}$ \\
\hline innonet B & $\begin{array}{l}\text { Innovationsnetzwerk Burgenland. Umsetzung von Innovationsmaßnahmen durch Information, Motivation } \\
\text { der Unternehmen. Verbesserung des Dienstleistungsangebots, Start-up-Initiative }\end{array}$ & & $\mathrm{x}$ & \\
\hline RESET & $\begin{array}{l}\text { Regionalentwicklung mit Schwerpunkt Erneuerbarer Energie und Gebäudetechnik. Zwei IZ mit den } \\
\text { Schwerpunkten 'erneuerbare Energie' bzw. 'Gebäudetechnik' entwickeln gemeinsames (Marketing-) } \\
\text { Konzept }\end{array}$ & & $\mathrm{x}$ & \\
\hline WTZ & $\begin{array}{l}\text { Werkstoff- und Technologie-Zentrum Obersteiermark. Es soll eine 'Region of Excellence für } \\
\text { Werkstofftechnologien' entwickelt werden durch: Werkstoffkompetenz-Datenbank, Beratungsnetzwerk, } \\
\text { Implementierung neuer Technologien, Weiterbildung, gemeinsames Marketing }\end{array}$ & & $\mathrm{x}$ & \\
\hline AROMA & $\begin{array}{l}\text { Impulsprojekt Ätherische Öle. Stärkung vorhandener F\&E-Kompetenz im Bereich 'innovativer Nutzung } \\
\text { von in der Region grundsätzlich kultivierb. Pflanzen' }\end{array}$ & & & $\mathrm{x}$ \\
\hline LEBRING & $\begin{array}{l}\text { strategische Kooperation mit der HTBLA Kaindorf (gemeinsames Konzept, Marketing, } \\
\text { Auftragsausarbeitung etc.) }\end{array}$ & & $\mathrm{x}$ & \\
\hline NIMM & $\begin{array}{l}\text { Erfolgssteigerung durch Engpassanalyse, Innovationsmanagement, Total Marketing Management, } \\
\text { Regionales Standort Management., PM }\end{array}$ & & $\mathrm{x}$ & \\
\hline WGW & $\begin{array}{l}\text { Technische Weiterentwicklung auf dem Gebiet der Robotik und Automationstechnik im WGM, } \\
\text { Öffentlichkeitsoffensive. Information über Möglichkeiten über Robotik und Automatisierungstechnik, } \\
\text { individuelle Beratung der Unternehmen; Schulung des Managements und Facharbeiter (gem. mit AMS } \\
\text { etc.); F\&E; Vernetzung und Öffentlichkeitsarbeit }\end{array}$ & & & $\mathrm{x}$ \\
\hline $\begin{array}{l}\text { Technologienetzwerk } \\
\text { Region Wr. Neustadt }\end{array}$ & Modell - Investoren, Venturer etc. bezüglich Gründungen und Technologisierung von Unternehmen & & $\mathrm{x}$ & \\
\hline REGkoop & $\begin{array}{l}\text { Kooperations- und Vernetzungsplattform IZ BREGENZERWALD. Aufwertung des IZ Bregenzerwald und } \\
\text { Schaffung eines Akteursnetzwerkes }\end{array}$ & & $\mathrm{x}$ & \\
\hline $\begin{array}{l}\text { Innovationsnetzwerk NÖ } \\
\text { Süd }\end{array}$ & $\begin{array}{l}\text { Entwicklung eines Konzeptes, mit dem Firmen, die aus IZ ausziehen, weiter beraten und betreut werden } \\
\text { sollen }\end{array}$ & & $\mathrm{x}$ & \\
\hline \multicolumn{5}{|c|}{ C: } \\
\hline Enermode & Modell für eine Enerige-plus-Region auf Basis erneuerbarer Energie im oberösterreichischen Ennstal & & $\mathrm{x}$ & \\
\hline e-motion & $\begin{array}{l}\text { Konzeption und Errichtung eines Roboter- Simulations- Labors im Technologie- und Dienstleis- } \\
\text { tungszentrum Donau-Böhmerwald in enger Zusammenarbeit mit der HTL Neufelden Automatisie- } \\
\text { rungstechnik und Know-How-Partnern aus der Wirtschaft. }\end{array}$ & & & $\mathrm{x}$ \\
\hline com.park & $\begin{array}{l}\text { Entwicklung von innovativen Instrumenten und Methoden für eine Standortentwicklung rund um die vier } \\
\text { Impulszentren im Mühlviertel (Ziel } 2 \text { Gebiet) mit einem begleitenden Marketingkonzept. }\end{array}$ & & $\mathrm{x}$ & \\
\hline IR Süd Ost & Innovationsregion Süd Ost & & $\mathrm{x}$ & \\
\hline
\end{tabular}




\begin{tabular}{|c|c|c|c|c|}
\hline Kurzbezeichnung & Beschreibung & $\begin{array}{c}\text { nach } \\
\text { innen }\end{array}$ & $\begin{array}{c}\text { nach } \\
\text { außen }\end{array}$ & $\begin{array}{c}\text { Infra- } \\
\text { struktur }\end{array}$ \\
\hline $\mathrm{B}^{2}$ - Next Step & $\mathrm{B}^{2}$ - Next Step & & $\mathrm{x}$ & \\
\hline K3 & Kunststoff Kompetenz-Netzwerk Kremstal & & $\mathrm{x}$ & \\
\hline ktla & Kremstaler Technische Lehr-Akademie & & $\mathrm{x}$ & \\
\hline Industrial Design & Industrial Design & & & $\mathrm{x}$ \\
\hline Sell-it & Sell - Innovative Technology & & $\mathrm{x}$ & \\
\hline Uttendorf & Entwicklungs- und Betriebskonzept Compoundierlabor Techno-Z Uttendorf & & & $\mathrm{x}$ \\
\hline Tech-iTV & $\begin{array}{l}\text { Internet TV Kompetenznetzwerk für technologische und wissenschaftliche Multimediaproduktionen und - } \\
\text { inhalte }\end{array}$ & & & $\mathrm{x}$ \\
\hline PROOF & $\begin{array}{l}\text { Prüf- und Forschungszentrum für elektronische Baugruppen und Komponenten in Kraftfahrzeugen und } \\
\text { Kraftfahrzeuganhängern }\end{array}$ & & & $\mathrm{x}$ \\
\hline InnoSpot & Impulszentren als regionale Innovationsdrehscheiben & & $\mathrm{x}$ & \\
\hline SUCCESS & Success with Systematic & & $\mathrm{x}$ & \\
\hline SWIFT & $\begin{array}{l}\text { Den Stress in der Wirtschaft innovativ nutzen und seine negativen Auswirkungen mit Forschung und } \\
\text { Technologie vermindern }\end{array}$ & & $\mathrm{x}$ & \\
\hline EssenZ & $\begin{array}{l}\text { Extrakte und natürliche Substanzen mit dem Schwerpunkt gesunde Ernährung - im Netzwerk in die } \\
\text { regionale Zukunft }\end{array}$ & & & $\mathrm{x}$ \\
\hline GkmiT & Gründung Kunststoff mit innovativen Technologien & & & $\mathrm{x}$ \\
\hline STARBORD & Start Across the Border Weinviertel & & $\mathrm{x}$ & \\
\hline RWB Steyr & Regionales Wissens- und Bildungsnetzwerk Steyr & & $\mathrm{x}$ & \\
\hline water-tec-net & water technology network & & $\mathrm{x}$ & \\
\hline BIO.COM.NET & Biotech Competence Network & & $\mathrm{x}$ & \\
\hline Lantech & Lantech - REGplus & & $\mathrm{x}$ & \\
\hline INNO@SIG & Innovationsüberleitung durch gemanagtes Experteninsourcing & & $\mathrm{x}$ & \\
\hline IMHOWA & $\begin{array}{l}\text { Impulszentrum - Holz - Waldviertel Entwicklung eines marktgerechten Produktangebotes der } \\
\text { Partnerbetriebe des Waldviertler Bau- \& Wohnstudios }\end{array}$ & & $\mathrm{x}$ & \\
\hline INCUBAT & Konzeption und Aufbau eines Business Incubation Programms & $\mathrm{x}$ & & \\
\hline LebLeb & $\begin{array}{l}\text { Entwicklung vom Prototypen zu seriengeeigneten Wohneinheiten auf Basis der Passivhaus- und } \\
\text { Nullenergietechnologie }\end{array}$ & & & $\mathrm{x}$ \\
\hline WBZ & Zentrum zur Förderung der Wettbewerbsfähigkeit von KMU's durch Nutzung der Ökoenergiekompetenz & & $\mathrm{x}$ & \\
\hline NIMM 2 & Netzwerk Impuls Management 2 & & $\mathrm{x}$ & \\
\hline \multicolumn{5}{|l|}{ 3. Runde } \\
\hline SimPLE.net & Network for Simple Produktion, Learning and Engineering & & $\mathrm{x}$ & \\
\hline
\end{tabular}




\begin{tabular}{|c|c|c|c|c|}
\hline Kurzbezeichnung & Beschreibung & $\begin{array}{c}\text { nach } \\
\text { innen }\end{array}$ & $\begin{array}{c}\text { nach } \\
\text { außen }\end{array}$ & $\begin{array}{c}\text { Infra- } \\
\text { struktur }\end{array}$ \\
\hline SIUS & $\begin{array}{l}\text { Innovation durch Kooperation von KMU, Großunternehmen, Forschungs- und Bildungseinrichtungen } \\
\text { mittels überbetrieblichem Ideenmanagement SIUS }\end{array}$ & & $\mathrm{x}$ & \\
\hline B-RIS & Regionale Innovationsstrategie für das Burgenland & & $\mathrm{x}$ & \\
\hline i-weg & $\begin{array}{l}\text { Industrielles Werkstoffbearbeitungszentrum Georgsberg / Eibiswald für die Fahrzeug- und } \\
\text { Luftfahrttechnologie }\end{array}$ & & & $\mathrm{x}$ \\
\hline Produktentwicklung & Zentrum für Produktentwicklung & & & $\mathrm{x}$ \\
\hline bio.com.net II & Ausbau des Forschungsverwertungsnetzwerks Life Sciences Tirol - Phase II & & $\mathrm{x}$ & \\
\hline TRANSFER & Aufbau einer Programmschiene des Ars Electronica Futurelab für KMU & & $\mathrm{x}$ & \\
\hline GDI Steyr & Gründer- und Diversifikations-Initiative Steyr 2010 & & $\mathrm{x}$ & \\
\hline hyper etal processing & hyper etal processing & & & $\mathrm{x}$ \\
\hline energycenter-net & $\begin{array}{l}\text { Netzwerk von Unternehmen, Institutionen aus F\&E und Bildung, } 2 \text { Technologiezentren, die sich auf das } \\
\text { Thema 'Erneuerbare Energien' konzentrieren, der regionale Schwerpunkt liegt in Wels Stadt / Wels Land, } \\
\text { Vöcklabruck und Weiz / Gleisdorf }\end{array}$ & & $\mathrm{x}$ & \\
\hline IQ²-Management & $\begin{array}{l}\text { Stärkung der Wettbewerbfähigkeit des nationalen Energie- und Bausektors durch kombinierte Anwendung } \\
\text { von Instrumenten und Methoden des Innovations-, QS-, Internationalisierungs- und Qualifizierungs- } \\
\text { Management }\end{array}$ & & $\mathrm{x}$ & \\
\hline NEE & Netzwerk Erneuerbare Energie & & $\mathrm{x}$ & \\
\hline Bio-Z & $\begin{array}{l}\text { Machbarkeitsstudie für ein Entwicklungs- und Anwendungszentrum regenerative Energien / Schwerpunkt } \\
\text { Biomasse }\end{array}$ & & & $\mathrm{x}$ \\
\hline rapidPRO & $\begin{array}{l}\text { Projekt zur Entwicklung, Aufbau und Betrieb eines Rapid Prototyping Anwendungs-Centers im Techno-Z } \\
\text { Ried }\end{array}$ & & & $\mathrm{x}$ \\
\hline ISB & $\begin{array}{l}\text { Innovate Smart Business: Projekt zur Unterstützung von Unternehmensgründern und zur Steigerung der } \\
\text { Gründungszahlen im Bereich Smart Business. Das Projekt soll nachhaltig den Gründern und TZs zur } \\
\text { Verfügung stehen und den TZs ein klares Profil im Bereich der Gründerunterstützung }\end{array}$ & $\mathrm{x}$ & & \\
\hline PIONIERE & $\begin{array}{l}\text { Projekt zur Implementierung von Planzenölen: Nachhaltige und Innovative Entwicklung einer regionalen } \\
\text { Wertschöpfungskette }\end{array}$ & & & $\mathrm{x}$ \\
\hline FH Region OÖ & Regionale Technologiezentren als Partner der Fachhochschulstudiengänge Oberösterreichs & & $\mathrm{x}$ & \\
\hline AREAmp3 & Neue Player für das regionale Innovations-System & & $\mathrm{x}$ & \\
\hline LANTECH2 & LANTECH2 & & $\mathrm{x}$ & \\
\hline ACCESS & Grenzüberschreitendes ACCESS Zentrum & & $\mathrm{x}$ & \\
\hline VIRGO & Virtuelles Gründerzentrum Oststeiermark & & $\mathrm{x}$ & \\
\hline Infocenter Bahninfrastruktur & Infocenter Bahninfrastruktur & & & $\mathrm{x}$ \\
\hline
\end{tabular}




\begin{tabular}{|c|c|c|c|c|}
\hline Kurzbezeichnung & Beschreibung & $\begin{array}{c}\text { nach } \\
\text { innen }\end{array}$ & $\begin{array}{c}\text { nach } \\
\text { außen }\end{array}$ & $\begin{array}{c}\text { Infra- } \\
\text { struktur }\end{array}$ \\
\hline AlpMobil & Einsatz alternativer mobiler Antriebstechnik im alpinen Bereich & & $\mathrm{x}$ & \\
\hline TRIS & Training in Innovation und Strategie & & $\mathrm{x}$ & \\
\hline inno hub & $\begin{array}{l}\text { developing an efficient regional hub for the integration of local technology centers in an international } \\
\text { innovation system }\end{array}$ & & $\mathrm{x}$ & \\
\hline \multicolumn{5}{|c|}{ e } \\
\hline PROOF 2 & $\begin{array}{l}\text { Entwicklung von Prüfmethoden und Einrichtung eines Prüf- und Kompatibilitätszentrums für die } \\
\text { Automobilindustrie, Drehscheibe für regionale Klein- und Mittelunternehmen, Beratungsfunktion für die } \\
\text { regionale und überregionale Wirtschaft (KMUs) }\end{array}$ & & & $\mathrm{x}$ \\
\hline Together & $\begin{array}{l}\text { Effizienzsteigerung beim Zugang zu Informationen für KMUs, Intensivierung der Kooperations- und } \\
\text { Netzwerkaktivitäten der Firmen im Verbund der TZs und der TZs als Regionaleinrichtungen, Matching von } \\
\text { Angebot und Nachfrage im Bereich Innovation- und Technologietransfer, Besserer Zugang zu nationalen } \\
\text { und internationalen Know-How Pools, Anbindung von spezialisierten KMUs an Technologiekooperationen } \\
\text { und wissenschaftliche Einrichtungen }\end{array}$ & & $\mathrm{x}$ & \\
\hline tech-lab & Aufbau von Laborinfrastruktur für Mechatronik und Oberflächenmesstechnik & & & $\mathrm{x}$ \\
\hline TZ Prüfregion OÖ & $\begin{array}{l}\text { Errichtung eines Prüftechnik Center mit dem Schwerpunkt Bauteilprüfung. Zweck des Prüfstandes ist es, } \\
\text { den Unternehmen der Region günstig innovative Prüftechnik vor Ort zur Verfügung zu stellen }\end{array}$ & & & $\mathrm{x}$ \\
\hline Q-net & $\begin{array}{l}\text { Kooperationsprojekt zur Sicherung von einheitlichen Qualitätsstandards in den Arbeitsabläufen der } \\
\text { Impulszentren des Netzwerkes OÖ }\end{array}$ & $\mathrm{x}$ & & \\
\hline $\begin{array}{l}\text { PNC-Park Networking } \\
\text { Carinthia }\end{array}$ & $\begin{array}{l}\text { Aufbau und Einführung eines kundenzentrierten, standortübergreifenden, informationstechnischen } \\
\text { Netzwerkes, mit dem Ziel, an allen Standorten von Impulszentren und für alle Kunden, die in der Strategie } \\
\text { festgelegten Leistungen - Incubating, Technologietransfer, Netzwerke, Regionalentwicklung - } \\
\text { flächendeckend, homogen und qualitativ hochwertig anbieten zu können }\end{array}$ & & $\mathrm{x}$ & \\
\hline
\end{tabular}

Quelle: FFG, Bundesministerium für Verkehr, Innovation und Technologie

NB: Die geförderten Projekte sind grau unterlegt. 


\subsection{Projektportfolio der Zentren}

Wir haben in Kapitel 5.2 die inhaltlich-strategische Orientierung der eingereichten bzw. geförderten Projekte betrachtet und feststellen können, dass zwei Drittel der Projekte auf die Region bezogen sind und überdies ein Drittel auf die Bereitstellung von Infrastruktur gerichtet ist, die überwiegend für Nutzer außerhalb des Zentrums von Bedeutung ist. Wir konnten daraus den recht klaren Schluss ziehen, dass sich die Manager dieser Zentren vom Immobilienmanager zum Regionalmanager verwandelt haben. Es liegt, unter diesem Gesichtspunkt der Transformation zum Regionalmanager, die Vermutung nahe, dass die Projekte aus dem RIF 2000 Programm in der Regel nicht die einzigen sind, die von den Impulszentren akquiriert und durchgeführt werden.

Wir sind dieser Frage nachgegangen und haben dabei entdecken können, dass im Grunde jedes Zentrum das eine oder andere Projekt betreibt. Tabelle 23 gibt hierzu eine umfassende Übersicht über das Projektportfolio von Impulszentren, die im Rahmen des Fördermoduls REGplus Projekte eingereicht bzw. gefördert bekommen haben. Darin wird insbesondere ersichtlich, dass es einen nicht unerheblichen Anteil an Zentren gibt, deren Projektportfolio beachtliche Ausmaße annimmt. Besonders typisch ist dies für die Bundesländer Oberösterreich, Steiermark und das Burgenland. Auch in Tirol gibt es eine starke Tendenz, wonach intermediäre Organisationen (darunter auch das im Rahmen von RIF 2000 geförderte AplusB-Zentrum CAST bzw. das Industrielle Kompetenzzentrum KMT) systematisch ihr Portfolio anreichern. Motiv ist hier die Sicherung des Fortbestands nach Auslaufen bzw. Reduktion der Finanzierung jener Aufgaben, deretwegen sie gegründet wurden.

In Tabelle 23 sind 48 Zentren bzw. Förderungswerber aufgelistet, davon sind 6 virtuelle Zentren, bei 9 liegen keine Angaben vor, eines wurde inzwischen geschlossen bzw. in die Entwicklungsagentur des Landes übergeführt, also gibt es bei 32 Zentren bzw. Förderungswerber Informationen über ihre sonstigen Projekte bzw. Projektbeteiligungen. Unter diesen befinden sich alle durch RIF 2000 geförderten Zentren bzw. solche, die an diesen Projekten beteiligt sind. Auf Basis dieser Informationen lassen sich einige interessante Beobachtungen machen bzw. Schlüsse ziehen.

- Auf 32 Zentren kommen über deutlich über 150 Beteiligungen an diversen Projekten einschließlich der REGplus und RIFupgrade Projekte.

- Es ist die Regel, dass Zentren an mehreren Projekten beteiligt sind. Die Vielfalt ist enorm und hat im Wesentlichen (i) mit einem ziemlich offenen Zielsystem der Zentren und (ii) mit der Verfügbarkeit von Förderangeboten zu tun. Einige Zentren verfügen über ein Portfolio von über einem Dutzend Projektbeteiligungen, die bis zu drei Jahre dauern und den Charakter eines gleichmäßigen Einkommensstroms aufweisen. Schwankungen werden personalseitig durch projektweise Anstellungen ausgeglichen. Es gehört offenbar zum Geschäftsmodell dieser Zentren, eine gewisse Zahl an Projekten am Laufen zu halten.

- Es gibt, wie immer, auch hier Konzentrationen: Die acht Zentren (26\%) mit den meisten Projektbeteiligungen (> 7) kommen allein für 88 Beteiligungen auf (64\%). Es sind dies drei aus der Steiermark (EIZ Weiz, Ökopark Hartberg, Telepark Bärnbach), vier aus Oberösterreich (TIC Steyr, TIZ Kirchdorf, TZ Braunau am Inn, TZ Salzkammergut / Attnang) und eines aus dem Burgenland, 
das im Wesentlichen alle anderen burgenländischen Zentren repräsentiert (TZ Eisenstadt / BIC). Was die Abwicklung der Projekte betrifft, so lassen sich hier zwei Modelle identifizieren: (i) Durchführung mit einem relativ größeren eigenem Team mit entsprechenden Projektmitarbeitern, z.B. EIZ Weiz, TZ Braunau, TIC Steyr oder (ii) überwiegend unter intensiver Heranziehung Dritter. Diese Dritten rekrutieren sich ihrerseits aus dem Kreis der Berater, aus den Firmen bzw. Mieter aus dem Zentrum (z.B. HTL ${ }^{48}$ ), aber auch Mitarbeiter aus der Gemeinde- bzw. Stadtverwaltung ${ }^{49}$.

- Gerade für die Zentren mit einer größeren Anzahl an Projekten hat sich zunehmend das Arbeits- und Organisationsprinzip 'Projekt' als dominantes Organisationsprinzip durchgesetzt: Es befinden sich immer ein paar Ideen in der Pipeline. Es werden Chancen für deren Finanzierung gesucht, wobei hier EU-geförderte Projekte (vor allem aus den Strukturfonds bzw. dem INTERREG Programm) eine herausragende Rolle spielen. Schließlich spielt die Tatsache, dass es spezialisierte Mitarbeiter gibt, eine nicht unerhebliche Rolle. Diese wollen / müssen beschäftigt werden und so gibt letztlich einen permanenten Druck, zusätzliche Einkommensquellen zu erschließen. Wir sehen diese Entwicklung durchaus ambivalent, weil sie einerseits als Ausdruck von Kontinuität und Nachhaltigkeit zu interpretieren ist ${ }^{50}$, andererseits aber die Gefahr besteht, dass hier ein mit öffentlichen Mitteln geschaffener Markt für intermediäre Dienstleistungen entsteht ${ }^{51}$.

- Wir können auch hier, diesmal unter dem Gesichtspunkt der Vernetzung mit anderen Zentren und Programmen und mit deren Zielgruppen abermals feststellen, dass die Mehrzahl der Zentren ihre Systembildungs- und Vernetzungsarbeit in hohem Maß geleistet haben. Sie beherrschen in der Regel das Geschäft des Akquirierens von Abwickelns von Projekten und haben diese Option nicht nur in ihr Geschäftsmodell integriert, sondern es bisweilen zum tragenden Prinzip ihres Geschäftsmodells gemacht. Meist gibt es 'Champions', die die anderen überragen. So auch hier: Ein Viertel der in RIF 2000 bzw. REGplus

48 Durch die Regionalisierung der Impulszentren in einigen Bundesländern ergibt sich - nicht ganz zufällig - eine systematische Verbindung zwischen den Impulszentren und den HTLs: Standortgemeinschaften, Lehrer als Firmeninhaber oder als Berater, Schüler als Projektmitarbeiter im Zuge ihrer Ausbildung.

49 In einem Zentrum ist das Gemeindeamt untergebracht. Dies erweckt zunächst den Eindruck eines Webfehlers, ähnlich wie Polizeistationen, Behindertenwerkstätten oder Fahrradreparaturwerkstätten. Im Falle des Gemeindeamts stellt sich aber ein sehr spezifischer positiver Effekt ein: Der Bürgermeister und der Amtsleiter (früher: 'Gemeindesekretär') arbeiten im selben Haus wie mit ihnen auch zehn kleinere engagierte Firmen. Dies ermöglicht den beiden Schlüsselpersonen der Gemeindepolitik einschließlich den Mitgliedern des Gemeinderates eine engere Tuchfühlung zur Wirtschaft im Allgemeinen und zur Hochtechnologie und Innovation im Besonderen, Konzepte, die in der Erfahrungswelt der genannten Akteure nicht immer realistisch besetzt sind.

50 Einen Hinweis auf die Bedeutung von Kontinuität und Nachhaltigkeit erhält man auch aus der Beobachtung, dass die virtuellen Impulszentren nicht wirklich überzeugen konnten und durchwegs durchgefallen sind. Sie tauchen überwiegend in Niederösterreich und Salzburg auf und drücken deren Erfolgsbilanz nicht unerheblich.

51 Wir beobachten eine solche Situation bei diversen quasi-institutionalisierten Initiativen und Programmen (insbesondere Clusterinitiativen, Kompetenzzentren), die nur teilfinanziert sind und die sich den restlichen Teil aus anderen Quellen erschließen müssen. Traditionell waren und sind dies private Mittel, häufig verstanden als Mitgliedsbeiträge. In jüngerer Zeit zeichnet sich eine Tendenz dahingehend ab, dass, weil vielfach die privaten Quellen nicht vermehrbar oder die öffentlichen Mittel besser zugänglich sind, die Ko-Finanzierung aus anderen öffentlichen Titeln erschlossen wird. 
aktiven Zentren sind in nahezu zwei Drittel (64\%) der Projekte beteiligt. Sie können als eine sehr spezifische Form von Plattform für überwiegend öffentlich finanzierte Projekte verstanden werden und damit auch als ein Intermediär, der für eine ganze Reihe von Programmen in irgendeiner Weise als Zielgruppe angesehen werden kann (aktuell: INTERREG, EUREGIO, protec INNO, protec netplus, CIR-CE, Kompetenzzentren, AplusB, Cluster, Strukturfondsprogramme (darunter auch das auf die Entwicklung des ländlichen Raums abzielende Programm Leader+), Uni:invent / Innovationsscout).

Tabelle 23 Projektportfolio von Förderwerbern im Fördermodul REGplus

\begin{tabular}{|c|c|}
\hline Förderungswerber & Projekte \\
\hline \multicolumn{2}{|l|}{ Burgenland } \\
\hline TZ Eisenstadt / BIC & $\begin{array}{ll}\text { 1. } & \text { REGplus Projekt START SMART B2 } \\
\text { 2. } & \text { START SMART Chance Ungarn } \\
\text { 3. } & \text { START SMART Mobile Business } \\
\text { 4. } & \text { DIANE - INTERREG IIIA } \\
\text { 5. } & \text { TEC PARK NET } \\
\text { 6. } & \text { RDA-net Ceda - INTERREG IIC } \\
\text { 7. } & \text { Teska } \\
\text { 8. } & \text { BAER - Building a European Region - Interreg IIIA } \\
\text { 9. } & \text { Trans-IT } \\
\text { 10. } & \text { I2E Infotech to Extend } \\
\text { 11. } & \text { CONET } \\
\text { 12. I2Net } \\
\text { 13. } & \text { REGplus Projekt INNONET B } \\
\end{array}$ \\
\hline TZ Güssing & $\begin{array}{ll}\text { 1. } & \text { TecParkNet } \\
\text { 2. } & \text { Diane } \\
\end{array}$ \\
\hline \multicolumn{2}{|l|}{ Kärnten } \\
\hline \multicolumn{2}{|l|}{ Niederösterreich } \\
\hline ACCESS Industrial Park Austria GmbH & $\mathrm{kA}$ \\
\hline ARGE Tulln & virtuelles Impulszentrum \\
\hline Holznetzwerk Management Vitis & virtuelles Impulszentrum \\
\hline Plattform für Innovationsmanagement & virtuelles Impulszentrum \\
\hline RIZ NÖ & $\mathrm{kA}$ \\
\hline RIZ NÖ-Süd & 1. $\quad$ REGplus Projekt GkmiT \\
\hline TBZ Groß-Siegharts & $\mathrm{kA}$ \\
\hline TFZ Wr. Neustadt & $\mathrm{kA}$ \\
\hline \multicolumn{2}{|l|}{ Oberösterreich } \\
\hline AEC Linz MuseumsgesmbH & 1. REGplus Projekt TRANSFER \\
\hline Gründer- und Technologiezentrum Wels & $\mathrm{kA}$ \\
\hline TDZ Donau - Böhmerwald & $\begin{array}{ll}\text { 1. } & \text { InterregIIIA Projekt "IC 48,5" } \\
\text { 2. } & \text { REGplus Projekt 'e-Motion' } \\
\text { 3. } & \text { REGplus FH Region OÖ } \\
\end{array}$ \\
\hline TDZ Ennstal & $\begin{array}{ll}\text { 1. } & \text { REGplus Projekt ENERMODE } \\
\text { 2. } & \text { BIOKERZE (Fabrik der Zukunft) } \\
\text { 3. } & \text { z-international } \\
\text { 4. } & \text { Strategieverdichtung oö. Ennstal } \\
\text { 5. } & \text { RIFupgrade Projekt TOGETHER } \\
\end{array}$ \\
\hline $\begin{array}{l}\text { TIZ Technologiezentrum Landl- } \\
\text { Grieskirchen }\end{array}$ & \begin{tabular}{|ll} 
1. & RIFupgrade Projekt TZ Prüfregion OÖ \\
2. & RIFupgrade Projekt TOGETHER \\
\end{tabular} \\
\hline TechCenter Linz-Winterhafen & $\begin{array}{ll}\text { 1. } & \text { sell-it } \\
\text { 2. } & \text { RIFupgrade Projekt tech lab } \\
\text { 3. } & \text { RIFupgrade Projekt Qualitätsmanagement Qnet } \\
\text { 4. } & \text { TMG als größter Mieter mit ihrem Portfolio } \\
\end{array}$ \\
\hline $\begin{array}{l}\text { Technologieregion Enns - St. Florian } \\
\text { Betriebsgesellschaft }\end{array}$ & $\mathrm{kA}$ \\
\hline
\end{tabular}




\begin{tabular}{|c|c|}
\hline Förderungswerber & Projekte \\
\hline TGZ Schärding & $\begin{array}{ll}\text { 1. } & \text { CITY NETZ } \\
\text { 2. } & \text { University of Southern Queensland } \\
\text { 3. } & \text { REGplus Projekt Prüfzentrum PROOF } \\
\text { 4. } & \text { REGplus Projekt IT-Lab: Transfer- und } \\
& \text { Applikationslabor für Informationstechnologien } \\
\end{array}$ \\
\hline TIC Steyr & $\begin{array}{ll}\text { 1. } & \text { protec netplus Projekt ADC Plus } \\
\text { 2. } & \text { CAR \& CARGOBIKE } \\
\text { 3. } & \text { K-TASS } \\
\text { 4. } & \text { REGplus Projekt GDI Steyr } \\
\text { 5. } & \text { NWGU II } \\
\text { 6. } & \text { RIO M1 } \\
\text { 7. } & \text { RIO M2 } \\
\text { 8. } & \text { RIO M5 } \\
\text { 9. } & \text { REGplus Projekt RWB } \\
\text { 10. } & \text { RIFupgrade Projekt together } \\
\end{array}$ \\
\hline TIZ Kirchdorf & $\begin{array}{ll}\text { 1. } & \text { REGplus Projekt hyper metal processing } \\
\text { 2. } & \text { protec netplus Projekt Entwicklung einer Virtuellen } \\
& \text { Firma im Spritzguss- Werkzeug- und Formenbau } \\
\text { 3. } & \text { virTOOeL } \\
\text { 4. } & \text { REGplus Projekt k3 } \\
\text { 5. } & \text { RIFupgrade Projekt TOGETHER } \\
\text { 6. } & \text { F\&E Ressourcen } \\
\text { 7. } & \text { Karriere mit Kunststoff } \\
\text { 8. } & \text { REGplus Projekt KTLA } \\
\text { 9. } & \text { MARIBOR } \\
\text { 10. } & \text { Von der Strategie zur Aktion } \\
\text { 11. } & \text { RIFupgrade Projekt Qualitätsmanagement Qnet } \\
\end{array}$ \\
\hline TZ Braunau/Inn & $\begin{array}{ll}\text { 1. } & \text { CITY NETZ } \\
\text { 2. } & \text { University of Southern Queensland } \\
\text { 3. } & \text { REGplus Projekt Prüfzentrum PROOF } \\
\text { 4. } & \text { RIFupgrade Projekt Prüfzentrum PROOF } 2 \\
\text { 5. } & \text { RIFupgrade Projekt TOGETHER } \\
\text { 6. } & \text { RIFupgrade Projekt Qualitätsmanagement Qnet } \\
\text { 7. } & \text { Breitbandnetzwerk Schärding - Passau } \\
\text { 8. } & \text { TechnologienetZwerk TZI-TZR-TGZ } \\
\text { 9. } & \text { RIFupgrade Projekt Qualitätsmanagement Qnet } \\
\text { 10. } & \text { Grenzüberschreitende Firmenvernetzung Braunau - } \\
\text { Töging } \\
\text { 11. } \\
\text { 12. Trormations- und Service-Zentrale } \\
\text { 13. } \text { Interreg-Projekt Euro-Schulen Call-Center Agent/In } \\
\text { 14. } & \text { Poseidon (Schul-Wirtschaftsprojekt Simbach- } \\
\text { Braunau/Inn - Citynetz) } \\
\text { 15. } \text { protec INNO Projekt weak ties } \\
\end{array}$ \\
\hline TZ Ried & $\begin{array}{ll}\text { 1. } & \text { CITY NETZ } \\
\text { 2. } & \text { University of Southern Queensland } \\
\text { 3. } & \text { REGplus Projekt Prüfzentrum PROOF } \\
\text { 4. } & \text { REGplus Projekt rapid pro } \\
\text { 5. } & \text { RIFupgrade Projekt TOGETHER } \\
\text { 6. } & \text { RIFupgrade Projekt Qualitätsmanagement Qnet } \\
\end{array}$ \\
\hline TZ Salzkammergut / Attnang & $\begin{array}{ll}\text { 1. } & \text { VC Anlage } \\
\text { 2. } & \text { REGplus Projekt water-tec-net } \\
\text { 3. } & \text { z-international } \\
\text { 4. } & \text { SWOT-SPOT } \\
\text { 5. } & \text { REGplus Projekt energycenter-net } \\
\text { 6. } & \text { Protec netplus Projekt O² Fischerei } \\
\text { 7. } & \text { Protec netplus Projekt HTS-SC goes East } \\
\text { 8. } & \text { RIFupgrade Projekt TOGETHER } \\
\end{array}$ \\
\hline
\end{tabular}




\begin{tabular}{|c|c|}
\hline Förderungswerber & Projekte \\
\hline TZ Salzkammergut / Gmunden & \begin{tabular}{|ll} 
1. & protec netplus HoliTe \\
2. & RIFupgrade Projekt TOGETHER \\
\end{tabular} \\
\hline \multicolumn{2}{|l|}{ Salzburg } \\
\hline Flachgauer Techno-GWZ Förderverein & virtuelles Impulszentrum \\
\hline TZ Pongau-Pinzgau-Lungau & 1. REGplus Projekt LUHIZ \\
\hline Verein Regionalentwicklung Pinzgau & virtuelles Impulszentrum \\
\hline \multicolumn{2}{|l|}{ Steiermark } \\
\hline AREA m Styria GmbH & 1. REGplus Projekt AREAmp3 \\
\hline $\begin{array}{l}\text { ARGE INNOVATION AUSTRIA } \\
\text { (Gmbh in Gründung) }\end{array}$ & $\mathrm{kA}$ \\
\hline EIZ Weiz & $\begin{array}{ll}\text { 1. } & \text { Avstrija-Slowenien } \\
\text { 2. } & \text { MATE } \\
\text { 3. I²-Net Interregionales Internationalisierungsnetzwerk } \\
\text { 4. } & \text { T.E.A.M. } \\
\text { 5. } & \text { GO BEST } \\
\text { 6. } & \text { IR Styria } \\
\text { 7. } & \text { REGplus Projekt Nimm } 2 \\
\text { 8. } & \text { Common Challenge } \\
\text { 9. } & \text { O.P.A. } \\
\text { 10. Eurosyn } \\
\text { 11. } \\
\text { 12n } \\
\text { 13. } \text { CANELLITAL-P } \\
\text { 14. Easy } \\
\text { 15. } \\
\text { 16EGplus Projekt Nimm } \\
\text { 17. Enent Office Paket } \\
\text { Energie } \\
\end{array}$ \\
\hline Gründer- \& Servicezentrum Fürstenfeld & $\begin{array}{ll}\text { 1. } & \text { I2net } \\
\text { 2. } & \text { IR Styria } \\
\text { 3. } & \text { REGplus Projekt IR Süd Ost } \\
\text { 4. } & \text { REGplus Projekt NIMM } \\
\text { 5. } & \text { REGplus Projekt NIMM2 } \\
\end{array}$ \\
\hline Innofinanz Graz & $\begin{array}{ll}\text { 1. } & 24 \text { Zentren } \\
\text { 2. } & \text { AplusB } \\
\text { 3. } & \text { Dachorganisation für zahlreiche Impulszentren } \\
\end{array}$ \\
\hline Ökopark Hartberg & $\begin{array}{ll}\text { 1. } & \text { REGplus Projekt EssenZ } \\
\text { 2. } & \text { REGplus Projekt AROMA } \\
\text { 3. } & \text { REGplus Projekt NIMM2 } \\
\text { 4. } & \text { REGplus TRIS } \\
\text { 5. } & \text { EUROSYN } \\
\text { 6. } & \text { Öko-Haus } \\
\text { 7. } & \text { Desiccant-Klimaanlage } \\
\text { 8. } & \text { Grüne Bioraffinerie } \\
\text { 9. } & \text { RENET Austria } \\
\text { 10. } & \text { Energieplan Hartberg } \\
\text { 11. } & \text { Chemisch-technische Pflanzennutzung }\end{array}$ \\
\hline Projektgemeinschaft WIP West & virtuelles Impulszentrum \\
\hline Technologiezentrum Deutschlandsberg & 1. RegPlus - Innovationsregion Süd-Ost \\
\hline Telepark Bärnbach & \begin{tabular}{|ll} 
2. & RegPlus - Innovationsregion Süd-Ost \\
3. & INTERREG III A, T.E.A.M. \\
4. & LEADER+ LAG Weststeiermark \\
5. & TeleReg Projekt Wirtschaftsraum Süd-West- \\
& Oststeiermark \\
6. & CADSES REVITAMIN INTERREG III B \\
7. & InServNet INTERREG III B CADSES \\
8. & protec netplus Projekt Logistik End-to-End-Solution \\
& durch den Einsatz von RFID-Komponenten
\end{tabular} \\
\hline TEZ Georgsberg-Stainz-St.Stefan Gmbh & 1. REGplus Projekt i-weg \\
\hline
\end{tabular}




\begin{tabular}{|c|c|}
\hline Förderungswerber & Projekte \\
\hline WPO - Wirtschaftspark Obersteiermark & 1. REGplus Projekt WTZ \\
\hline $\begin{array}{l}\text { Zentrum für angewandte Technologie } \\
\text { Leoben / Außeninstitut der MU Leoben }\end{array}$ & $\begin{array}{ll}\text { 1. } & \text { AplusB } \\
\text { 2. } & \text { REPplus Projekt Produktentwicklung } \\
\text { 3. } & \text { protec INNO Projekt Die Ideenmaschine - Marketing } \\
& \text { \& Sales } \\
\text { 4. } & \text { protec INNO Projekt better ideas for better business } \\
\text { 5. } & \text { protec netplus Projekt Entwicklung von } \\
& \text { maßgeschneiderten Polymer-Werkstoffen für } \\
& \text { Präzisions-Mikrospritzgussteile }\end{array}$ \\
\hline $\begin{array}{l}\text { WGM - Wirtschaftspark / } \\
\text { Gründerzentrum Mürzzuschlag }\end{array}$ & 1. $\quad$ Robotik, Automationstechnik \\
\hline \multicolumn{2}{|l|}{ Tirol } \\
\hline CAST-Center Tirol & \begin{tabular}{|ll} 
1. & AplusB \\
2. & Uni:invent \\
3. & REPplus Projekt BIO.COM.NET
\end{tabular} \\
\hline Innovationszentrum Reutte & 1. IKA Ingenieur Kolleg Automatisierungstechnik \\
\hline IZ Lantech & \begin{tabular}{|ll} 
1. & REPplus Projekt LANTECH \\
2. & REPplus Projekt LANTECH 2 \\
3. & INTERREG III A \\
\end{tabular} \\
\hline Kompetenzzentrum Medizin GmbH & $\begin{array}{ll}\text { 1. } & \text { REPplus Projekt BIO.COM.NET } \\
\text { 2. } & \text { REPplus Projekt BIO.COM.NET } 2 \\
\text { 3. } & \text { CLUSTER Life Science Tirol } \\
\text { 4. } & \text { NaDiNe } \\
\end{array}$ \\
\hline TZ Tech-Tirol & von Tiroler Zukunftsstiftung übernommen \\
\hline \multicolumn{2}{|l|}{ Vorarlberg } \\
\hline Impulszentrum Bregenzerwald & $\mathrm{kA}$ \\
\hline PRISMA Holding Dornbirn & 1. REPplus Projekt INCUBAT \\
\hline \multicolumn{2}{|l|}{ Wien } \\
\hline SIG Wien-Simmering & $\mathrm{kA}$ \\
\hline
\end{tabular}

Quelle: FFG, Verband der Technologiezentren Österreichs, www.vto.at, Homepages der einzelnen Zentren. NB: Die im Rahmen von REGplus geförderten Zentren sind grau unterlegt.

Ein besonders dichtes Netzwerk an Beziehungen zwischen Zentren weist die Steiermark auf. Dies ist auch erklärte Politik sowohl der beteiligten Zentren selbst als auch der Entwicklungsagentur des Landes, der Steirischen Wirtschaftsförderungsgesellschaft. Beweggrund für diese Zusammenarbeit ist Spezialisierung und Arbeitsteilung. Jedes Zentrum soll ein Kompetenzprofil aufweisen, welches auf der einen Seite die erforderliche Sachkompetenz ('Branchenkenntnis') sicherstellt und andererseits über die unmittelbare Umgebung (halbe bis ganze Autostunde) hinaus seine insoweit erworbene Spezialisierung auch für andere Regionen zur Verfügung stellt. Konzeptueller Rahmen und gleichzeitig Arbeitsgrundlage sind mehrjährige Projekte. Wir finden in Tabelle 24 eine illustrative Übersicht über die Beteiligungen und Partnerschaften im Rahmen von REGplus-, INTERREG- sowie Projekten des Landes. Zu dieser Strategie der Vernetzung durch Arbeitsteilung hat nicht unwesentlich das REGplusProgramm beigetragen. 
Tabelle 24 Verflechtungen von Zentren und Projekten am Beispiel Ost- und Südsteiermark

\begin{tabular}{|c|c|c|c|c|c|c|c|}
\hline & I2net & T.E.A.M. & $\begin{array}{c}\text { IR } \\
\text { Styria } \\
\end{array}$ & $\begin{array}{c}\text { IR Süd } \\
\text { Ost }\end{array}$ & NIMM & NIMM2 & $\begin{array}{l}\text { Common } \\
\text { Challenge }\end{array}$ \\
\hline $\begin{array}{l}\text { Technologiezentrum } \\
\text { Deutschlandsberg }\end{array}$ & $\mathrm{x}$ & & & $\mathrm{x}$ & & & \\
\hline W.E.I.Z & $\mathrm{x}$ & $\mathrm{x}$ & $\mathrm{X}$ & $\mathrm{x}$ & $\mathrm{x}$ & $\mathrm{x}$ & $\mathrm{x}$ \\
\hline Telepark Bärnbach & $\mathrm{x}$ & $\mathrm{x}$ & $\mathrm{X}$ & $\mathrm{x}$ & & & \\
\hline $\mathrm{BIC}$ & $\mathrm{x}$ & & & & & & \\
\hline $\begin{array}{l}\text { Gründer- \& Service- } \\
\text { zentrum Fürstenfeld }\end{array}$ & $\mathrm{x}$ & & $\mathrm{x}$ & $\mathrm{x}$ & $\mathrm{x}$ & $\mathrm{x}$ & \\
\hline $\begin{array}{l}\text { Bildungszentrum } \\
\text { Deutschlandsberg }\end{array}$ & & & $\mathrm{x}$ & & & & \\
\hline $\begin{array}{l}\text { Freiraum Business } \\
\text { Center }\end{array}$ & & & $\mathrm{X}$ & $\mathrm{X}$ & & & \\
\hline $\begin{array}{l}\text { Regionale Impuls- } \\
\text { gesellschaft Süd-Ost }\end{array}$ & & & & & & $\mathrm{x}$ & \\
\hline $\begin{array}{l}\text { Wirtschaftskammer } \\
\text { Steiermark }\end{array}$ & $\mathrm{x}$ & & & & & & $\mathrm{x}$ \\
\hline Ökopark Hartberg & & & & & $\mathrm{x}$ & $\mathrm{x}$ & \\
\hline
\end{tabular}

Quelle: Homepages der diversen Organisationen bzw. Projekte

Was oben am Beispiel der Steiermark vorgeführt wurden, lässt sich in unterschiedlichen Graden verallgemeinern und in anderen Kontexten wieder finden.

- Managementverbünde. In der RIZ-Holding gibt es Untergesellschaften, die von den Mitarbeitern der RIZ-Holding mitbetreut werden. Die Zentren in Braunau, Ried im Innkreis und Schärding werden von einem Geschäftsführer geführt, ähnliche Strukturen gibt es im Mühlviertel bzw. im Salzkammergut. In Vorarlberg ist die Fa. PRISMA durchgängig präsent.

- Eigentümerschaft. RIZ-Holding und PRISMA können noch einmal erwähnt werden zusammen mit der Steirischen Innofinanz und der oberösterreichischen TMG bzw. der burgenländischen Zentrenlandschaft, in Teilen auch Wien mit dem Zentrum für Innovation und Technologie, wo gemeinsame, übergreifende Eigentümerstrukturen vorherrschen.

- Projektverbünde bzw. Marketingverbünde. Immer wieder werden Projekte gemeinsam durchgeführt. Neben dem oben beschriebenen steirischen Projektcluster ist hier Oberösterreich zu erwähnen, die über die landesfinanzierten Projekte und später mit Bundesförderung durchgeführten Projekte zusammen arbeiten. Bei der Errichtung von Infrastrukturen (z.B. in Landl-Grieskirchen) kam es zu Kooperationen mit anderen Zentren in Bezug auf die Vermarktung des an einem Ort aufgebauten Prüfzentrums.

- Leiter von Impulszentren mit Zweitberuf. Wir kehren hier bewusst die positiven Seiten jener Gruppe von Leitern von Impulszentren hervor, die zugleich einem anderen Beruf nachgehen: Bürgermeister, Amtsleiter, Lehrer an einer HTL, Berater. Der positive Effekt dieser Anordnung besteht darin, dass es hier Zugang zu anderen Ressourcen und Infrastrukturen gibt, fallweise auch zu Märkten, was vor allem dann von Vorteil ist, wenn Investitionen getätigt wurden, die Mindestgrößen erfordern.

- Zusammenarbeit aus anderen Projekten. Diese gibt es, das bindende Glied ist in der Regel der Leiter des Zentrums. Sein Netzwerk und seine Reputation sind 
daher hier die Schlüsselfaktoren. Ein gelungenes erstes Projekt ist umgekehrt ein Schlüsselfaktor für die Reputation des Zentrumsleiters. Dies ist natürlich nur scheinbar ein Plus, weil auf der andere Seite der Medaille die Verletzbarkeit des Zentrums aufgeprägt ist.

\section{Schlussfolgerungen}

- Wir fassen die bisherigen Ausführungen über die Bewirtschaftung von Projektportfolios noch einmal zusammen und halten fest, dass (i) die Zentrumsmanager nicht nur in ihrer Selbstdefinition, sondern (ii) auch auf Ebene der bei REGplus eingereichten Projekte den Schritt zum Regionalmanager ${ }^{52}$ weithin vollzogen haben. Wir können davon ausgehen, dass es keinen Manager mehr gibt (außer, deklarierter Weise, jene des privaten Inkubators), der sich nicht einer regionalwirtschaftlichen bzw. regionalpolitischen Ausrichtung verpflichtet fühlt.

- Sowohl unter dem Imperativ der regionalen Vernetzung und der Wahrnehmung regionalpolitischer Agenden als auch unter dem Druck zur Selbstfinanzierung greifen die Zentren in besonderem Ausmaß auf verfügbare Förder- und Finanzierungstöpfe des Landes, des Bundes und vor allem der EU zurück. Sie befinden sich dadurch alsbald in einer Situation, in der sie zu überwiegenden Teilen mit der Abwicklung der Projekte beschäftigt sind und landen damit in einer systematisch prekären Finanzsituation, die vor allem durch die Notwendigkeit der KoFinanzierung verursacht wird.

- 'Vernetzung', verstanden hier als durch öffentliche Förderprogramme vorangetriebene Kooperation zwischen Impulszentren, entpuppt sich damit zusehends als Unterfangen, welches in einem Spannungsfeld angesiedelt ist, das (i) durch KoFinanzierung durch Dritte (meist Land bzw. EU, selten Träger), (ii) Darstellung überhöhter Kosten und (iii) Selbstausbeutung (der Manager) geprägt ist ${ }^{53}$.

- Am Ende dieser Diskussion und nach fünf Jahren Laufzeit des RIF 2000 Programms ist fairer Weise die Frage zu stellen, ob es noch zweckmäßig ist, Impulszentren zum de facto ${ }^{54}$ ausschließlichen Adressaten eines Förderprogramms zu machen, welches sich in seinen wesentlichen Belangen unbemerkt in ein innovations- und technologieorientiertes Regionalentwicklungsprogramm verwandelt hat, bei dem Impulszentren die Funktion eines Intermediärs einnehmen.

- Eine halbe Antwort lässt sich an dieser Stelle geben: Es waren und sind Impulszentren, die sich nach und nach in kleinere und größere Regionalagenturen transformiert haben. Sie haben meist ein kontingentes Profil, es ist also den Umständen geschuldet, was sie anbieten und wie sie es $\operatorname{tun}^{55}$. Wesentlich waren und sind dabei die diversen Förderprogramme, aus denen sie Projekte eingeworben haben.

52 Wir weisen noch einmal darauf hin, dass wir hier nicht die einschlägige Programmschiene meinen.

53 Letzteres ist insofern sehr wahrscheinlich, weil es sich hier um überdurchschnittlich motivierte Personen handelt, die im Allgemeinen mit schlecht strukturierten Zielen arbeiten und daher notorisch zur Selbstausbeutung und Überforderung neigen.

54 In den Richtlinien sind zwar als Förderungswerber Impulszentren oder vergleichbare Infrastruktureinrichtungen angeführt. Abgesehen von virtuellen Impulszentren haben sich de facto mit ganz wenigen Ausnahmen aber nur 'echte' Impulszentren am RIF 2000 Programm beteiligt.

55 Die 'Umstände' sind hier im Wesentlichen die deutlich veränderte Förderlandschaft und die damit verbundene Inanspruchnahme der Förderangebote. 
Hätten sie andere Projekte eingeworben oder hätten sie dies mit anderen Partnern getan, wären mit hoher Wahrscheinlichkeit andere Profile entstanden ${ }^{56}$.

- Diese Transformation der Impulszentren zu Regionalentwicklungsagenturen mit Technologie- und Innovationsorientierung wirft natürlich die Frage der Fortsetzung des RIF 2000 Programms auf. Diese Frage kann an dieser Stelle aber nicht erschöpfend beantwortet werden. Ein Beitrag zur Klärung dieser Frage besteht aber darin, dass wir deutliche Evidenz dafür haben, dass es einen gewissen Bedarf nach lokal-regionalen Zugangs- und Verteilstrukturen gibt. Die besseren Impulszentren erfüllen diesen Bedarf. Man kann dies daran erkennen, dass sie in der lokalen Wirtschaft gut integriert sind und dass man sie ruft. Dies geleistet zu haben, ist ein wesentliches Verdienst der Förderlinie REGplus.

\subsection{Das Netzwerk der österreichischen Technologie-, Impuls- und Gründerzentren und ihrer Dienstleistungen}

Es gibt zur Zeit (August 2005) in Österreich 104 Technologie-, Impuls- und Gründerzentren. Diese Zentren verstehen sich als Einrichtungen sowohl für die Unterstützung bei der Gründung neuer und technologie-orientierter Unternehmen als auch als innovative, regionale Impulsgeber. Tabelle 26 gibt einen detaillierten Überblick über die Landschaft, aufgeschlüsselt nach Bundesländern, Gründungsjahr, eingemieteten Firmen und Fläche. Darüber hinaus wird eine Übersicht über die wichtigsten Dienstleistungen, die von Impulszentren typischer Weise erwartet werden, gegeben.

- Gründerberatung

- Geschäftspläne

- Personalsuche

- Technologieberatung

- Kooperationsvermittlung

- Finanzierungsberatung

- Organisationsberatung

Wir gehen im Folgenden einigen Fragen nach, die vor allem aus der Zielsetzung von RIF 2000 her motiviert sind. Es ist dies vor allem die Frage nach dem Dienstleistungsspektrum, dazu zu entwickeln und zu fördern die wesentliche Zielsetzung von RIF 2000, insbesondere des Förderungsmoduls REGplus war. Dieses richtet sich zum Einen nach innen und zielt auf die Unterstützung von Unternehmen bei ihrer Gründung sowie ihrer weiteren Entwicklung ab, also etwa Personalsuche, Technologieberatung, Partnersuche / Kooperationsvermittlung, Finanzierungsberatung, Organisationsberatung. Alle diese Dienstleistungen können im Prinzip gleichermaßen auch nach außen hin angeboten werden. Die nähere Analyse der in Tabelle 26 dargestellten Daten zeitigen hier einige interessante Details. So erkennen wir in Abbildung 4 sehr deutlich, dass die Zentren mit einem recht umfangreichen Dienstleistungsspektrum ausgestattet sind: Etwa die Hälfte der Zentren verfügt über ein komplettes Dienst-

56 Diese Bemerkung ist auch als Kritik an der gemeinhin üblichen Förderpraxis zu verstehen. Sie zielt primär auf das Projekt ab, in zweiter Instanz auf den Träger des Projekts, und dann bevorzugt auf die Kapazität, das Projekt umzusetzen. Ein dritte Betrachtungsebene, nämlich die Rolle und Position des zu fördernden Projekts im Kontext anderer Projekte und Kompetenzen, also im Portfolio, kommt systematisch zu kurz. Diese wird bestenfalls unter dem Gesichtspunkt der 'Doppelförderung' einer Analyse unterzogen. 
leistungsangebot. Bei deutlich mehr als zwei Drittel (71\%) kann man davon ausgehen, dass die eine oder andere Dienstleistung fehlt. Im Wesentlichen ist die Palette aber weitgehend komplett.

Abbildung 4 Verteilung der Dienstleistungen der Impulszentren

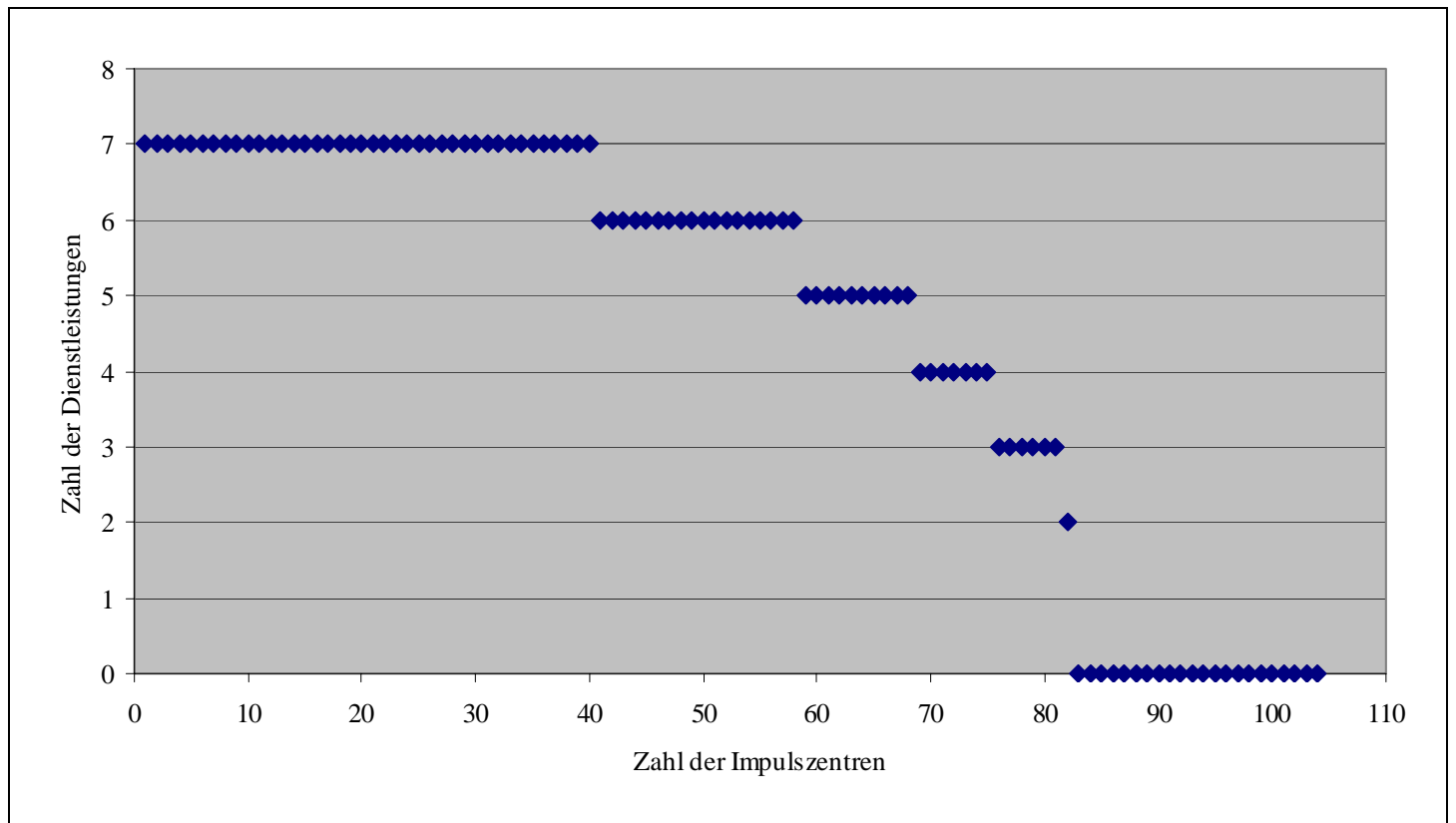

Quelle: Verband der Technologiezentren Österreich, www.vto.at, Homepages der diversen Zentren, NB: '0 Dienstleistungen' entspricht 'keine Angaben'.

Tabelle 25 gibt die inhaltliche Struktur des Dienstleistungsspektrums wieder. Hier zeigt sich, dass alle Zentren Gründungsberatung anbieten. Gleichermaßen sind die Unterstützung in der Entwicklung von Geschäftsplänen (91\%) und in der Vermittlung von Kooperationen (90\%) hoch im Kurs. Etwas weniger häufig, aber immer noch in hohem Maße im Dienstleistungsspektrum der Impulszentren vorhanden, sind Finanzierungsberatung (85\%), Technologieberatung (84\%), Organisationsberatung (76\%) und, etwas abgeschlagen, aber immer noch von 2 von 3 Zentren angeboten, Personalsuche.

Tabelle 25 Verteilung der Dienstleistungen in den Impulszentren

\begin{tabular}{|l|c|c|c|c|c|c|c|}
\hline $\begin{array}{l}\text { Dienst- } \\
\text { leistung }\end{array}$ & $\begin{array}{c}\text { Gründer- } \\
\text { beratung }\end{array}$ & $\begin{array}{c}\text { Geschäfts- } \\
\text { pläne }\end{array}$ & $\begin{array}{c}\text { Personal- } \\
\text { suche }\end{array}$ & $\begin{array}{c}\text { Technologie- } \\
\text { beratung }\end{array}$ & $\begin{array}{c}\text { Kooperations- } \\
\text { vermittlung }\end{array}$ & $\begin{array}{c}\text { Finanzierungs- } \\
\text { beratung }\end{array}$ & $\begin{array}{c}\text { Organisations- } \\
\text { beratung }\end{array}$ \\
\hline Zahl & 82 & 75 & 53 & 69 & 74 & 70 & 62 \\
\hline Anteil & $100 \%$ & $91 \%$ & $65 \%$ & $84 \%$ & $90 \%$ & $85 \%$ & $76 \%$ \\
\hline
\end{tabular}

Quelle: Verband der Technologiezentren Österreich, www.vto.at, Homepages der diversen Zentren, NB: Unter 'Anteil' verstehen wir hier den Anteil an jenen Zentren, die in wenigstens eine Dienstleistung bekannt gegeben haben. Da 22 keine Angaben gemacht haben, ist die Bezugsgröße 82 von 104.

Insgesamt kann man in einer ersten Einschätzung davon ausgehen, dass die Zentren hervorragend mit Dienstleistungen ausgestattet sind, die für Gründung und Wachstum von Unternehmen wichtig sind. Im Einzelnen zeichnen sich die Zentren durch eine große Offenheit aus:

- Überregionale Impulszentren. Nicht immer werden die Dienstleistungen vor Ort angeboten, z.B. werden in Niederösterreich Büros betrieben, in denen 
Sprechstunden abgehalten werden. Im Burgenland oder in Salzburg werden bestimmte Leistungen von der jeweiligen Zentrale in Eisenstadt bzw. in der Stadt Salzburg angeboten.

- Vermittlung von Dienstleistungen. Nicht nur wird die Erbringung bestimmter Dienstleistungen an das überregionale (Mutter)Zentrum delegiert, es werden vielfach auch Dritte herangezogen, wobei die Entwicklungsagentur des jeweiligen Landes und die Wirtschaftskammer bevorzugter Partner sind, letztere vor allem bei Fragen der Gründung und bei Behördenangelegenheiten.

- Offenheit gegenüber externen Klienten. In wenigstens drei von vier Fällen werden die Dienstleistungen nicht nur den Mietern, sondern auch externen Unternehmen angeboten.

Wir verhehlen nicht eine gewisse kritische Distanz gegenüber dem Broker-Modell, bei dem Dienstleistungen Dritter vermittelt werden. Dieses setzt einen gewissen Grad an Explizitheit der Problemstellung voraus und ist stark auf Problemlösung hin orientiert - man bearbeitet dann eben einen Fall, ein Projekt oder bedient einen Klienten in einer bestimmten Frage. Die Betreuung von Unternehmen in Impulszentren ist oft das des 'Kümmerns', also auch dann da zu sein, wenn nichts anliegt, nicht nur Probleme zu lösen, sondern auch Chancen heranzutragen usw.. So oder so, ein Mindestmaß an Präsenz und Kontinuität ist von den Zentren aufrecht zu erhalten ${ }^{57}$. Die Welt der Projekte verleidet dieses Verhalten.

\section{Schlussfolgerungen}

- Das Dienstleistungsportfolio des größten Teils der Impulszentren ist im Wesentlichen komplett. Dort, wo die Dienstleistungen nicht selbst angeboten werden, bieten die Zentren eine Weitervermittlung an.

- Die Zentren halten also die entsprechenden Dienstleistungen selber vor oder bedienen sich Dritter. Dies sind typischer Weise das überregionale Zentrum, die Entwicklungsagentur des Landes bzw. die Wirtschaftskammer. Darüber hinaus und für spezielle Fragen werden Fachleute aus dem privaten Sektor herangezogen.

- Insgesamt betrachten wir dieses Netzwerk von Impulszentren, zusammen mit anderen verwandten und komplementären Anbietern und Zubringern (Förderagenturen, Entwicklungsagenturen, spezialisierte Intermediäre (z.B. Clusterinitiativen ${ }^{58}$ ), Wirtschaftskammern, private Berater, Banken) als hinlänglich dicht, sowohl was die räumliche als auch die thematische Dichte betrifft. Es ist

57 "Die wichtigsten Leute will ich im eigenen Haus haben. Berater sind oft nicht sehr hilfreich."

58 Clusterinitiativen sind nicht nur als Programme zu betrachten, sondern auch und zunehmend mehr als eigenständige Organisationen, so etwa in der Steiermark, in Tirol und neuerdings auch im 'Clusterland' Oberösterreich. Dort hat sich die Entwicklungsagentur des Landes, die TMG, im Wesentlichen identisch reproduziert und eine eigene Clustergesellschaft abgespalten. Wir wagen hier die Prognose, dass diese neue Gesellschaft im Laufe der Zeit ihr Dienstleistungsspektrum verändern, in Summe aber anreichern wird. Ursache dieser Anreicherung wird der Zwang zur Eigenfinanzierung sein, der dazu führen wird, dass man nach Opportunitäten greifen wird, die sich vor allem im öffentlichen Raum auftun werden. Typische Fälle sind hier die Übernahme von Aufgaben öffentlicher Einrichtungen (der Landesregierung, von Ministerien, der EU-Kommission) bzw. die Akquisition von Förderprojekten, bevorzugt INTERREG, Strukturfondsprojekte, ERANET-Projekte etc. sowie die in diesem Bericht mehrfach angesprochenen nationalen Programme. 
möglich, innerhalb einer Autostunde Zugang zu wesentlichen Unterstützungsangeboten bzgl. Unternehmensgründung und Unternehmensentwicklung zu erhalten. Im Gegenzug ist es möglich, den Einzugsbereich der einzelnen Impulszentren so klein zu halten, dass ein hinreichend enger informeller Kontakt zu den Zielgruppen möglich ist.

- Die Tatsache, dass Impulszentren einerseits Dienstleistungen für Unternehmen außerhalb der Zentren anbieten und andererseits auf dritte Anbieter zurückgreifen, kann als überzeugender Beweis dafür gesehen werden, dass Impulszentren tatsächlich Agentur- bzw. Brokerfunktionen einnehmen und in unterschiedlichen Graden auch leben. Daraus ergibt sich ein hohes Maß an Entkopplung der Bewirtschaftung von Gebäuden einerseits und dem Anbieten von Unterstützungsdienstleistungen.

- Uneinheitlich ist dagegen die Qualität des Dienstleistungsportfolios. Diese hängt wesentlich von den Zentrumsmanagern (Qualifikation, Verfügbarkeit, Netzwerk, Persönlichkeitsprofil) ab. Es gibt inzwischen eine Reihe von Bemühungen, einheitliche Qualitätsstandards zu entwickeln und einzuführen. Das RIF 2000 Programm hat hier sowohl was die Qualität auf Ebene der Programmabwicklung betrifft, als auch hinsichtlich der Förderung spezifischer, auf die Anhebung der Qualität gerichteter Projekte, zweifellos Verdienste erworben. 
Tabelle 26 Technologie-, Impuls- und Gründerzentren in Österreich

\begin{tabular}{|c|c|c|c|c|c|c|c|c|c|c|c|c|c|}
\hline Bundesland & Zentrum & $\begin{array}{l}\text { Grün- } \\
\text { dung }\end{array}$ & Firmen & $\begin{array}{c}\text { Arbeits- } \\
\text { plätze }\end{array}$ & $\begin{array}{l}\text { vermietbare } \\
\text { Fläche }\end{array}$ & $\begin{array}{c}\text { davon } \\
\text { Bürofläche }\end{array}$ & $\begin{array}{l}\text { Gründer- } \\
\text { beratung }\end{array}$ & $\begin{array}{l}\text { Geschäfts- } \\
\text { pläne }\end{array}$ & $\begin{array}{l}\text { Personal- } \\
\text { suche }\end{array}$ & $\begin{array}{l}\text { Technologie- } \\
\text { beratung }\end{array}$ & $\begin{array}{c}\text { Kooperations- } \\
\text { vermittlung }\end{array}$ & $\begin{array}{c}\text { Finanzierungs- } \\
\text { beratung }\end{array}$ & $\begin{array}{c}\text { Organisations- } \\
\text { beratung }\end{array}$ \\
\hline \multirow[t]{6}{*}{ Burgenland } & $\begin{array}{l}\text { Technologiezentrum } \\
\text { Eisenstadt }\end{array}$ & 1997 & 90 & 1.153 & 27.306 & 27.306 & $x$ & $\mathrm{x}$ & $\mathrm{x}$ & $x$ & $\mathrm{x}$ & $\mathrm{x}$ & $\mathrm{x}$ \\
\hline & $\begin{array}{l}\text { Technologiezentrum } \\
\text { Güssing }\end{array}$ & 2002 & 22 & $\mathrm{kA}$ & 3.371 & 3.371 & $\mathrm{x}$ & $\mathrm{x}$ & $\mathrm{x}$ & $\mathrm{x}$ & $\mathrm{x}$ & $\mathrm{x}$ & $\mathrm{x}$ \\
\hline & $\begin{array}{l}\text { Technologiezentrum } \\
\text { Jennersdorf }\end{array}$ & 2001 & 14 & 64 & 2.323 & 1.450 & $\mathrm{x}$ & $\mathrm{x}$ & $\mathrm{x}$ & $\mathrm{x}$ & $\mathrm{x}$ & & $\mathrm{x}$ \\
\hline & \begin{tabular}{|l|} 
Technologiezentrum \\
Mittelburgenland
\end{tabular} & 2002 & 14 & $\mathrm{kA}$ & 4.167 & 4.167 & $\mathrm{x}$ & $\mathrm{x}$ & $\mathrm{x}$ & $\mathrm{x}$ & $\mathrm{x}$ & $\mathrm{x}$ & $\mathrm{x}$ \\
\hline & \begin{tabular}{|l} 
Technologiezentrum \\
Neusiedl am See
\end{tabular} & 2003 & 10 & $\mathrm{kA}$ & 8.025 & & $\mathrm{x}$ & $\mathrm{x}$ & $\mathrm{x}$ & $\mathrm{x}$ & $\mathrm{x}$ & $\mathrm{x}$ & $\mathrm{x}$ \\
\hline & $\begin{array}{l}\text { Technologiezentrum } \\
\text { Pinkafeld }\end{array}$ & 2000 & 10 & $\mathrm{kA}$ & 2.507 & 1.507 & $\mathrm{x}$ & $\mathrm{x}$ & $\mathrm{x}$ & $\mathrm{x}$ & $\mathrm{x}$ & $\mathrm{x}$ & $\mathrm{x}$ \\
\hline \multirow[t]{8}{*}{ Kärnten } & \begin{tabular}{|l|} 
Impulszentrum \\
Arnoldstein
\end{tabular} & 1999 & & $\mathrm{kA}$ & 3.246 & 1.300 & $\mathrm{x}$ & $\mathrm{x}$ & $\mathrm{x}$ & $\mathrm{x}$ & $\mathrm{x}$ & & $\mathrm{x}$ \\
\hline & \begin{tabular}{|l|} 
Impulszentrum \\
Oberkärnten
\end{tabular} & $\mathrm{kA}$ & $\mathrm{kA}$ & $\mathrm{kA}$ & $\mathrm{kA}$ & $\mathrm{kA}$ & $\mathrm{kA}$ & $\mathrm{kA}$ & $\mathrm{kA}$ & $\mathrm{kA}$ & $\mathrm{kA}$ & $\mathrm{kA}$ & $\mathrm{kA}$ \\
\hline & Impulszentrum St. Veit & $\mathrm{kA}$ & $\mathrm{kA}$ & $\mathrm{kA}$ & $\mathrm{kA}$ & $\mathrm{kA}$ & $\mathrm{kA}$ & $\mathrm{kA}$ & $\mathrm{kA}$ & $\mathrm{kA}$ & $\mathrm{kA}$ & $\mathrm{kA}$ & $\mathrm{kA}$ \\
\hline & \begin{tabular}{|l|} 
Impulszentrum \\
Völkermarkt
\end{tabular} & 1995 & 12 & 86 & 1.865 & 868 & $\mathrm{x}$ & $\mathrm{x}$ & $\mathrm{x}$ & $\mathrm{x}$ & $\mathrm{x}$ & & $\mathrm{x}$ \\
\hline & \begin{tabular}{|l|} 
Impulszentrum \\
Wolfsberg
\end{tabular} & $\mathrm{kA}$ & kA & $\mathrm{kA}$ & $\mathrm{kA}$ & $\mathrm{kA}$ & $\mathrm{kA}$ & $\mathrm{kA}$ & $\mathrm{kA}$ & $\mathrm{kA}$ & $\mathrm{kA}$ & $\mathrm{kA}$ & $\mathrm{kA}$ \\
\hline & $\begin{array}{l}\text { Lakeside Science and } \\
\text { Technology Park }\end{array}$ & 2004 & 14 & $\mathrm{kA}$ & 28.000 & 8.500 & $\mathrm{x}$ & $\mathrm{x}$ & $\mathrm{x}$ & $\mathrm{x}$ & & $\mathrm{x}$ & \\
\hline & $\begin{array}{l}\text { Technologiepark } \\
\text { Klagenfurt }\end{array}$ & 1992 & & 4.325 & 4.325 & & $\mathrm{x}$ & $\mathrm{x}$ & $\mathrm{x}$ & $\mathrm{x}$ & $\mathrm{x}$ & $\mathrm{x}$ & $\mathrm{x}$ \\
\hline & \begin{tabular}{|l} 
Technologiepark Villach \\
\end{tabular} & 2001 & 70 & 350 & 24.500 & 20.000 & $\mathrm{x}$ & $\mathrm{x}$ & $\mathrm{x}$ & $\mathrm{x}$ & $\mathrm{x}$ & $\mathrm{x}$ & $\mathrm{x}$ \\
\hline \multirow[t]{4}{*}{$\begin{array}{l}\text { Nieder- } \\
\text { österreich }\end{array}$} & \begin{tabular}{|l|} 
Business- und \\
Innovationszentrum St. \\
Pölten
\end{tabular} & 2001 & 29 & $\mathrm{kA}$ & 5.300 & 5.300 & $\mathrm{x}$ & & $\mathrm{x}$ & $\mathrm{x}$ & $\mathrm{x}$ & $\mathrm{x}$ & $\mathrm{x}$ \\
\hline & \begin{tabular}{|l} 
Gründer- und \\
Beratungszentrum \\
Gmünd \\
\end{tabular} & 1996 & 12 & 42 & 700 & 700 & $\mathrm{x}$ & & $\mathrm{x}$ & & $\mathrm{x}$ & & \\
\hline & \begin{tabular}{|l} 
Gründer- und \\
Innovationszentrum \\
Blue Danube Park
\end{tabular} & $\mathrm{kA}$ & 44 & 60 & 2.050 & & $\mathrm{x}$ & $\mathrm{x}$ & $\mathrm{x}$ & & $\mathrm{x}$ & $\mathrm{x}$ & $\mathrm{x}$ \\
\hline & $\begin{array}{l}\text { RIZ Gründerzentrum } \\
\text { Amstetten }\end{array}$ & 2000 & 17 & 60 & 1.680 & 1.130 & $\mathrm{x}$ & $\mathrm{x}$ & $\mathrm{kA}$ & $\mathrm{x}$ & $\mathrm{x}$ & $\mathrm{x}$ & $\mathrm{x}$ \\
\hline
\end{tabular}




\begin{tabular}{|c|c|c|c|c|c|c|c|c|c|c|c|c|c|}
\hline Bundesland & Zentrum & $\begin{array}{l}\text { Grün- } \\
\text { dung }\end{array}$ & Firmen & \begin{tabular}{|c|}
$\begin{array}{c}\text { Arbeits- } \\
\text { plätze }\end{array}$ \\
\end{tabular} & \begin{tabular}{c|}
$\begin{array}{c}\text { vermietbare } \\
\text { Fläche }\end{array}$ \\
\end{tabular} & \begin{tabular}{c|} 
davon \\
Bürofläche \\
\end{tabular} & $\begin{array}{l}\text { Gründer- } \\
\text { beratung }\end{array}$ & $\begin{array}{l}\text { Geschäfts- } \\
\text { pläne }\end{array}$ & $\begin{array}{c}\text { Personal- } \\
\text { suche }\end{array}$ & $\begin{array}{l}\text { Technologie- } \\
\text { beratung }\end{array}$ & $\begin{array}{c}\text { Kooperations- } \\
\text { vermittlung }\end{array}$ & $\begin{array}{c}\begin{array}{c}\text { Finanzierungs- } \\
\text { beratung }\end{array} \\
\end{array}$ & $\begin{array}{c}\text { Organisations- } \\
\text { beratung }\end{array}$ \\
\hline & $\begin{array}{l}\text { RIZ Gründerbüro } \\
\text { Gänserndorf }\end{array}$ & 2003 & 0 & $\mathrm{kA}$ & 34,92 & 34,92 & $x$ & $\mathrm{x}$ & $\mathrm{kA}$ & $\mathrm{kA}$ & $\mathrm{kA}$ & $\mathrm{x}$ & $\mathrm{kA}$ \\
\hline & $\begin{array}{l}\text { RIZ Gründerzentrum } \\
\text { Hollabrunn }\end{array}$ & 2001 & 8 & $\mathrm{kA}$ & 715 & 490 & $\mathrm{x}$ & $\mathrm{x}$ & $\mathrm{kA}$ & $\mathrm{x}$ & $\mathrm{x}$ & $\mathrm{x}$ & $\begin{array}{l}\mathrm{x} \\
\end{array}$ \\
\hline & $\begin{array}{l}\text { RIZ Gründerzentrum } \\
\text { Krems }\end{array}$ & 2000 & 19 & $\mathrm{kA}$ & 2.300 & 1.050 & $\mathrm{x}$ & $\mathrm{x}$ & $\mathrm{kA}$ & $\mathrm{x}$ & $\mathrm{kA}$ & $\mathrm{x}$ & $\mathrm{x}$ \\
\hline & $\begin{array}{l}\text { RIZ Gründerbüro } \\
\text { Mistelbach } \\
\end{array}$ & $\mathrm{kA}$ & 0 & $\mathrm{kA}$ & 0 & 0 & $\mathrm{x}$ & $\mathrm{x}$ & $\mathrm{kA}$ & $\mathrm{kA}$ & $\mathrm{kA}$ & $\mathrm{x}$ & $\mathrm{kA}$ \\
\hline & $\begin{array}{l}\text { RIZ Gründerzentrum } \\
\text { Piestingtal }\end{array}$ & 1997 & 4 & 9 & 166 & 90 & $\mathrm{x}$ & $\mathrm{x}$ & kA & $\mathrm{kA}$ & $\mathrm{x}$ & $\mathrm{x}$ & $\mathrm{x}$ \\
\hline & $\begin{array}{l}\text { RIZ Gründerzentrum } \\
\text { Bucklige Welt }\end{array}$ & 1997 & 4 & kA & 290 & 290 & $\mathrm{x}$ & $\mathrm{x}$ & $\mathrm{kA}$ & $\mathrm{x}$ & $\mathrm{x}$ & $\mathrm{x}$ & $\mathrm{x}$ \\
\hline & $\begin{array}{l}\text { RIZ Gründerzentrum } \\
\text { Schwarzatal }\end{array}$ & 1998 & 8 & $\mathrm{kA}$ & 617 & 300 & $\mathrm{x}$ & $\mathrm{x}$ & (x) & $\mathrm{x}$ & $\mathrm{x}$ & $\mathrm{x}$ & $\mathrm{x}$ \\
\hline & $\begin{array}{l}\text { RIZ Gründerzentrum } \\
\text { Triestingtal }\end{array}$ & 2003 & 13 & 27 & 624 & 412 & $\mathrm{kA}$ & $\mathrm{kA}$ & $\mathrm{kA}$ & $\mathrm{kA}$ & $\mathrm{kA}$ & $\mathrm{kA}$ & $\mathrm{kA}$ \\
\hline & $\begin{array}{l}\text { RIZ Gründerzentrum } \\
\text { Waidhofen/Ybbs }\end{array}$ & 2000 & 11 & 25 & 895 & 895 & $\mathrm{x}$ & $\mathrm{x}$ & $\mathrm{kA}$ & $\mathrm{x}$ & $\mathrm{x}$ & $\mathrm{x}$ & $\mathrm{kA}$ \\
\hline & $\begin{array}{l}\text { RIZ Gründerzentrum } \\
\text { Wr. Neustadt }\end{array}$ & 1988 & 17 & $\mathrm{kA}$ & 1.568 & 1.248 & $\mathrm{x}$ & $\mathrm{x}$ & $\mathrm{kA}$ & $\mathrm{kA}$ & $\mathrm{x}$ & $\mathrm{x}$ & $\mathrm{kA}$ \\
\hline & $\begin{array}{l}\text { Technologie- und } \\
\text { Bildungszentrum Groß } \\
\text { Siegharts }\end{array}$ & 1998 & 6 & 20 & 1180 & 880 & $\mathrm{x}$ & $\mathrm{x}$ & $\mathrm{x}$ & $\mathrm{x}$ & $\mathrm{x}$ & $\mathrm{x}$ & $\mathrm{x}$ \\
\hline & $\begin{array}{l}\text { Technologie- und } \\
\text { Forschungszentrum Wr. } \\
\text { Neustadt } \\
\end{array}$ & 2000 & 10 & 160 & 7.100 & 2.500 & $\mathrm{x}$ & $\mathrm{x}$ & $\mathrm{x}$ & $\mathrm{x}$ & $\mathrm{x}$ & $\mathrm{x}$ & $\mathrm{x}$ \\
\hline & $\begin{array}{l}\text { concorde technology } \\
\text { center schwechat }\end{array}$ & 2003 & 8 & 30 & 6.000 & \begin{tabular}{|l|}
6.000 \\
\end{tabular} & $\mathrm{x}$ & $\mathrm{x}$ & $\mathrm{x}$ & $\mathrm{x}$ & $\mathrm{x}$ & $x$ & $\mathrm{x}$ \\
\hline \multirow[t]{4}{*}{$\begin{array}{l}\text { Ober- } \\
\text { österreich }\end{array}$} & $\begin{array}{l}\text { Business- und } \\
\text { Innovationszentrum } \\
\text { Wels }\end{array}$ & 2002 & 4 & 300 & 20.000 & 10.000 & $\mathrm{x}$ & $\mathrm{x}$ & $\mathrm{x}$ & $\mathrm{x}$ & $\mathrm{x}$ & $\mathrm{x}$ & $\mathrm{x}$ \\
\hline & \begin{tabular}{|l|} 
Gründer- und \\
Technologiezentrum \\
Wels \\
\end{tabular} & 1996 & 50 & 205 & 5.800 & 5.800 & $\mathrm{x}$ & $\mathrm{x}$ & $\mathrm{x}$ & $\mathrm{x}$ & $\mathrm{x}$ & $\mathrm{x}$ & $\mathrm{x}$ \\
\hline & $\begin{array}{l}\text { Logistikzentrum Enns- } \\
\text { Hafen }\end{array}$ & 2000 & 10 & $\mathrm{kA}$ & 808 & 807 & $\mathrm{kA}$ & $\mathrm{kA}$ & $\mathrm{kA}$ & $\mathrm{kA}$ & $\mathrm{kA}$ & $\mathrm{kA}$ & $\mathrm{kA}$ \\
\hline & $\begin{array}{l}\text { Softwarepark Schloß } \\
\text { Hagenberg }\end{array}$ & 1998 & 40 & 600 & 8.350 & 8.000 & $\mathrm{x}$ & $\mathrm{kA}$ & $\mathrm{x}$ & $\mathrm{kA}$ & $\mathrm{x}$ & $\mathrm{kA}$ & $\mathrm{kA}$ \\
\hline
\end{tabular}




\begin{tabular}{|c|c|c|c|c|c|c|c|c|c|c|c|c|c|}
\hline Bundesland & Zentrum & $\begin{array}{l}\text { Grün- } \\
\text { dung }\end{array}$ & Firmen & $\begin{array}{c}\begin{array}{c}\text { Arbeits- } \\
\text { plätze }\end{array} \\
\end{array}$ & \begin{tabular}{c|}
$\begin{array}{c}\text { vermietbare } \\
\text { Fläche }\end{array}$ \\
\end{tabular} & \begin{tabular}{|c|} 
davon \\
Bürofläche
\end{tabular} & $\begin{array}{l}\text { Gründer- } \\
\text { beratung }\end{array}$ & $\begin{array}{l}\text { Geschäfts- } \\
\text { pläne }\end{array}$ & $\begin{array}{l}\text { Personal- } \\
\text { suche }\end{array}$ & $\begin{array}{l}\text { Technologie- } \\
\text { beratung }\end{array}$ & $\begin{array}{c}\text { Kooperations- } \\
\text { vermittlung }\end{array}$ & $\begin{array}{l}\text { Finanzierungs- } \\
\text { beratung }\end{array}$ & $\begin{array}{l}\text { Organisations- } \\
\text { beratung }\end{array}$ \\
\hline & $\begin{array}{l}\text { Technologie- und } \\
\text { Dienstleistungszentrum } \\
\text { Donau-Böhmerwald / } \\
\text { Haslach bzw. Neufelden }\end{array}$ & 2002 & 17 & 60 & 1.891 & 1.891 & $\mathrm{x}$ & $\mathrm{x}$ & $\mathrm{kA}$ & $\mathrm{x}$ & $\mathrm{x}$ & $\mathrm{x}$ & $\mathrm{x}$ \\
\hline & $\begin{array}{l}\text { TechCenter Linz- } \\
\text { Winterhafen }\end{array}$ & 2002 & 63 & 370 & 12.800 & 12.200 & $\mathrm{x}$ & $\mathrm{x}$ & $\mathrm{x}$ & $\mathrm{x}$ & $\mathrm{x}$ & $\mathrm{x}$ & $\mathrm{x}$ \\
\hline & $\begin{array}{l}\text { Technologiezentrum } \\
\text { Braunau }\end{array}$ & 1993 & 23 & $\mathrm{kA}$ & 3.105 & 3.105 & $\mathrm{x}$ & $\mathrm{x}$ & $\mathrm{x}$ & $\mathrm{x}$ & $\mathrm{x}$ & $\mathrm{x}$ & $\mathrm{x}$ \\
\hline & \begin{tabular}{|l|} 
Technologiezentrum \\
Ried im Innkreis \\
\end{tabular} & 2000 & 20 & $\mathrm{kA}$ & 2.800 & 2.800 & $\mathrm{x}$ & $\mathrm{x}$ & $\mathrm{x}$ & $\mathrm{x}$ & $\mathrm{x}$ & $\mathrm{x}$ & $\mathrm{x}$ \\
\hline & $\begin{array}{l}\text { Technologie- und } \\
\text { Gründerzentrum } \\
\text { Schärding }\end{array}$ & 2000 & 17 & $\mathrm{kA}$ & kA & kA & $\mathrm{x}$ & $\mathrm{x}$ & $\mathrm{x}$ & $\mathrm{x}$ & $\mathrm{x}$ & $\mathrm{x}$ & $\mathrm{x}$ \\
\hline & $\begin{array}{l}\text { Technologie- und } \\
\text { Innovationszentrum } \\
\text { Kirchdorf }\end{array}$ & 2002 & 21 & 89 & 2.791 & \begin{tabular}{|l|}
1.197 \\
\end{tabular} & $\mathrm{x}$ & $\mathrm{kA}$ & $\mathrm{kA}$ & $\mathrm{x}$ & $\mathrm{x}$ & $\mathrm{x}$ & $\mathrm{kA}$ \\
\hline & $\begin{array}{l}\text { Technologie- und } \\
\text { Innovationszentrum } \\
\text { Landl-Grieskirchen }\end{array}$ & 2004 & 37 & 80 & 3.354 & 1.257 & $\mathrm{x}$ & $\mathrm{x}$ & $\mathrm{kA}$ & $\mathrm{x}$ & $\mathrm{x}$ & $\mathrm{x}$ & $\mathrm{kA}$ \\
\hline & $\begin{array}{l}\text { Technologie- und } \\
\text { Innovationszentrum St. } \\
\text { Florian }\end{array}$ & 1999 & 22 & 70 & 5.600 & 5.200 & $\mathrm{x}$ & $\mathrm{x}$ & $\mathrm{kA}$ & $\mathrm{x}$ & $\mathrm{x}$ & $\mathrm{x}$ & $\mathrm{kA}$ \\
\hline & $\begin{array}{l}\text { Technologiezentrum } \\
\text { Freistadt-Mühlviertel } \\
\end{array}$ & 1998 & 15 & 67 & 1.670 & 1.504 & $\mathrm{x}$ & $\mathrm{x}$ & $\mathrm{kA}$ & $\mathrm{x}$ & $\mathrm{kA}$ & $\mathrm{x}$ & $\mathrm{x}$ \\
\hline & $\begin{array}{l}\text { Technologiezentrum } \\
\text { Perg } \\
\end{array}$ & 2003 & 21 & 70 & 3.000 & 1.800 & $\mathrm{x}$ & $\mathrm{x}$ & $\mathrm{kA}$ & $\mathrm{x}$ & $\mathrm{x}$ & $\mathrm{x}$ & $\mathrm{x}$ \\
\hline & $\begin{array}{l}\text { Technologiezentrum } \\
\text { Freistadt } \\
\end{array}$ & 1998 & 15 & 67 & 1670 & 1.504 & $\mathrm{x}$ & $\mathrm{x}$ & $\mathrm{kA}$ & $\mathrm{x}$ & $\mathrm{kA}$ & $\mathrm{x}$ & $\mathrm{x}$ \\
\hline & $\begin{array}{l}\text { Technologiezentrum } \\
\text { Gmunden }\end{array}$ & 2000 & 30 & 65 & 1.500 & 1.500 & $\mathrm{x}$ & $\mathrm{x}$ & $\mathrm{x}$ & $\mathrm{x}$ & $\mathrm{x}$ & $\mathrm{kA}$ & $\mathrm{x}$ \\
\hline & $\begin{array}{l}\text { Technologiezentrum } \\
\text { Bad Ischl }\end{array}$ & 2004 & 17 & $\mathrm{kA}$ & 1.640 & 1.640 & $\mathrm{x}$ & $\mathrm{x}$ & $\mathrm{x}$ & $\mathrm{x}$ & $\mathrm{x}$ & $\mathrm{kA}$ & $\mathrm{x}$ \\
\hline & \begin{tabular}{|l|} 
Technologiezentrum \\
Attnang-Puchheim
\end{tabular} & 2000 & 26 & 72 & 1.500 & 1.500 & (x) & (x) & & $\mathrm{x}$ & $\mathrm{x}$ & $\mathrm{x}$ & (x) \\
\hline & \begin{tabular}{|l|} 
Technology and \\
Innovation Center Steyr
\end{tabular} & 2001 & 18 & kA & 2.960 & $\mathrm{kA}$ & $\mathrm{x}$ & $\mathrm{x}$ & $\mathrm{x}$ & $\mathrm{x}$ & $\mathrm{x}$ & $\mathrm{x}$ & $\mathrm{x}$ \\
\hline Salzburg & Techno-Z Salzburg & 1988 & 102 & $\mathrm{kA}$ & 24.890 & 24.890 & $\mathrm{x}$ & $\mathrm{x}$ & $\mathrm{x}$ & $\mathrm{kA}$ & $\mathrm{x}$ & $\mathrm{x}$ & $\mathrm{x}$ \\
\hline & Techno-Z Bischofshofen & 1990 & 24 & 200 & 12.471 & 2.499 & $\mathrm{x}$ & $\mathrm{x}$ & $\mathrm{x}$ & $\mathrm{x}$ & $\mathrm{x}$ & $\mathrm{x}$ & $\mathrm{x}$ \\
\hline & \begin{tabular}{|l|} 
Techno-Z Mariapfarr \\
\end{tabular} & 1999 & 8 & 36 & 2.238 & 696 & $\mathrm{x}$ & $\mathrm{x}$ & $\mathrm{x}$ & $\mathrm{x}$ & $\mathrm{x}$ & $\mathrm{x}$ & $\mathrm{x}$ \\
\hline & Techno-Z Pfarrwerfen & 1998 & 3 & 10 & 587 & 587 & $\mathrm{x}$ & $\mathrm{x}$ & $\mathrm{x}$ & $\mathrm{x}$ & $\mathrm{x}$ & $\mathrm{x}$ & $\mathrm{x}$ \\
\hline & \begin{tabular}{|l|} 
Techno-Z Saalfelden \\
\end{tabular} & 1992 & 12 & 60 & 3.129 & 1.165 & $\mathrm{x}$ & $\mathrm{x}$ & $\mathrm{x}$ & $\mathrm{x}$ & $\mathrm{x}$ & $\mathrm{x}$ & $\mathrm{x}$ \\
\hline
\end{tabular}




\begin{tabular}{|c|c|c|c|c|c|c|c|c|c|c|c|c|c|}
\hline Bundesland & Zentrum & $\begin{array}{l}\text { Grün- } \\
\text { dung }\end{array}$ & Firmen & $\begin{array}{c}\text { Arbeits- } \\
\text { plätze }\end{array}$ & $\begin{array}{c}\begin{array}{c}\text { vermietbare } \\
\text { Fläche }\end{array} \\
\end{array}$ & \begin{tabular}{|c|} 
davon \\
Bürofläche
\end{tabular} & $\begin{array}{l}\text { Gründer- } \\
\text { beratung }\end{array}$ & $\begin{array}{l}\text { Geschäfts- } \\
\text { pläne }\end{array}$ & $\begin{array}{l}\text { Personal- } \\
\text { suche }\end{array}$ & $\begin{array}{l}\text { Technologie- } \\
\text { beratung }\end{array}$ & $\begin{array}{c}\text { Kooperations- } \\
\text { vermittlung }\end{array}$ & $\begin{array}{l}\text { Finanzierungs- } \\
\text { beratung }\end{array}$ & $\begin{array}{l}\text { Organisations- } \\
\text { beratung }\end{array}$ \\
\hline & Techno-Z Uttenorf & 2002 & 3 & 90 & 5.340 & 1.525 & $\mathrm{x}$ & $x$ & $\mathrm{x}$ & $x$ & $\mathrm{x}$ & $\mathrm{x}$ & $\mathrm{x}$ \\
\hline & Techno-Z Zell am See & 1992 & 16 & 25 & 795 & 519 & $\mathrm{x}$ & $\mathrm{x}$ & $\mathrm{x}$ & $\mathrm{x}$ & $\mathrm{x}$ & $\mathrm{x}$ & $\mathrm{x}$ \\
\hline \multirow[t]{21}{*}{ Steiermark } & \begin{tabular}{|l|} 
A.i.Z \\
Unternehmerzentrum
\end{tabular} & 1989 & 21 & $\mathrm{kA}$ & $\mathrm{kA}$ & $\mathrm{kA}$ & $\mathrm{kA}$ & $\mathrm{kA}$ & $\mathrm{kA}$ & $\mathrm{kA}$ & $\mathrm{kA}$ & $\mathrm{kA}$ & $\mathrm{kA}$ \\
\hline & $\begin{array}{l}\text { Impulszentrum } \\
\text { Grambach }\end{array}$ & 1994 & 34 & 170 & 5.600 & $\mathrm{kA}$ & $\mathrm{x}$ & $\mathrm{x}$ & $\mathrm{kA}$ & $\mathrm{x}$ & $\mathrm{x}$ & $\mathrm{kA}$ & $\mathrm{kA}$ \\
\hline & $\begin{array}{l}\text { Impulszentrum Graz- } \\
\text { West }\end{array}$ & 1986 & 12 & 70 & 1.000 & 1.000 & $\mathrm{x}$ & $\mathrm{x}$ & $\mathrm{kA}$ & $\mathrm{kA}$ & $\mathrm{x}$ & $\mathrm{kA}$ & $\mathrm{kA}$ \\
\hline & Impulszentrum Liezen & 1996 & 12 & 90 & 6.000 & 1.500 & $\mathrm{x}$ & $\mathrm{kA}$ & $\mathrm{kA}$ & $\mathrm{kA}$ & $\mathrm{x}$ & $\mathrm{x}$ & $\mathrm{kA}$ \\
\hline & \begin{tabular}{|l|} 
Impulszentrum \\
Niklasdorf \\
\end{tabular} & 1990 & 15 & 65 & 6.000 & 4.000 & $\mathrm{x}$ & $\mathrm{kA}$ & $\mathrm{kA}$ & $\mathrm{x}$ & $\mathrm{x}$ & $\mathrm{kA}$ & $\mathrm{kA}$ \\
\hline & $\begin{array}{l}\text { Impulszentrum } \\
\text { Radkersburg }\end{array}$ & 2001 & 3 & 4 & 1.000 & 150 & $\mathrm{x}$ & $\mathrm{x}$ & $\mathrm{kA}$ & $\mathrm{x}$ & $\mathrm{x}$ & $\mathrm{x}$ & $\mathrm{kA}$ \\
\hline & \begin{tabular}{|l|} 
Impulszentrum \\
Unterpremstetten
\end{tabular} & 2000 & 17 & $\mathrm{kA}$ & 13.600 & kA & $\mathrm{kA}$ & $\mathrm{kA}$ & $\mathrm{kA}$ & $\mathrm{kA}$ & $\mathrm{kA}$ & $\mathrm{kA}$ & $\mathrm{kA}$ \\
\hline & \begin{tabular}{|l|} 
Impulszentrum \\
Auersbach \\
\end{tabular} & 2001 & 8 & $\mathrm{kA}$ & 3.191 & 534 & $\mathrm{x}$ & $\mathrm{x}$ & $\mathrm{x}$ & $\mathrm{x}$ & $\mathrm{x}$ & $\mathrm{x}$ & $\mathrm{x}$ \\
\hline & \begin{tabular}{|l|} 
Innovationszentrum \\
Zeltweg (in Gründung)
\end{tabular} & & & & & & & & & & & & \\
\hline & Logistik Center Leoben & 1999 & 14 & $\mathrm{kA}$ & 3.815 & 2.870 & $\mathrm{x}$ & $\mathrm{x}$ & $\mathrm{x}$ & $\mathrm{x}$ & $\mathrm{x}$ & $\mathrm{x}$ & $\mathrm{x}$ \\
\hline & Ökopark Hartberg & 1998 & 20 & & & & & & & & & & \\
\hline & Science Park Graz & 2002 & 10 & $\mathrm{kA}$ & $\mathrm{kA}$ & $\mathrm{kA}$ & $\mathrm{kA}$ & kA & $\mathrm{kA}$ & $\mathrm{kA}$ & $\mathrm{kA}$ & $\mathrm{kA}$ & $\mathrm{kA}$ \\
\hline & \begin{tabular}{|l|} 
Start-up Center \\
Reininghaus (AplusB)
\end{tabular} & 2003 & 17 & $\mathrm{kA}$ & $\mathrm{kA}$ & $\mathrm{kA}$ & $\mathrm{kA}$ & $\mathrm{kA}$ & $\mathrm{kA}$ & $\mathrm{kA}$ & $\mathrm{kA}$ & $\mathrm{kA}$ & $\mathrm{kA}$ \\
\hline & $\begin{array}{l}\text { Technologiezentrum } \\
\text { Deutschlandsberg }\end{array}$ & 2000 & 9 & $\mathrm{kA}$ & 720 & 490 & $\mathrm{kA}$ & $\mathrm{kA}$ & $\mathrm{kA}$ & $\mathrm{kA}$ & $\mathrm{kA}$ & $\mathrm{kA}$ & $\mathrm{kA}$ \\
\hline & \begin{tabular}{|l|} 
Technologiezentrum \\
Kapfenberg
\end{tabular} & 1993 & 7 & 100 & 5.700 & 1.400 & $\mathrm{x}$ & $\mathrm{x}$ & $\mathrm{x}$ & $\mathrm{x}$ & $\mathrm{x}$ & $\mathrm{x}$ & $\mathrm{x}$ \\
\hline & \begin{tabular}{|l|} 
Technologie- und \\
Gründerpark Rosental
\end{tabular} & 2001 & $\mathrm{kA}$ & $\mathrm{kA}$ & 182 & 182 & $\mathrm{kA}$ & kA & $\mathrm{kA}$ & kA & $\mathrm{kA}$ & kA & $\mathrm{kA}$ \\
\hline & $\begin{array}{l}\text { Technologie- und } \\
\text { Innovationszentrum } \\
\text { Graz } \\
\end{array}$ & 1986 & 12 & 70 & 1.000 & 1.000 & $\mathrm{x}$ & $\mathrm{x}$ & & & & & \\
\hline & \begin{tabular}{|l|} 
Technologie- und \\
Wirtschaftspark Lebring \\
\end{tabular} & 2000 & 21 & 100 & 3.700 & 2.500 & $\mathrm{x}$ & $\mathrm{x}$ & $\mathrm{kA}$ & $\mathrm{x}$ & $\mathrm{x}$ & $\mathrm{kA}$ & $\mathrm{kA}$ \\
\hline & Telepark Bärnbach & 2001 & 15 & $\mathrm{kA}$ & $\mathrm{kA}$ & $\mathrm{kA}$ & $\mathrm{kA}$ & $\mathrm{kA}$ & $\mathrm{kA}$ & $\mathrm{kA}$ & $\mathrm{kA}$ & $\mathrm{kA}$ & $\mathrm{kA}$ \\
\hline & $\begin{array}{l}\text { Weizer Energie } \\
\text { Innovationszentrum }\end{array}$ & 1999 & 10 & $\mathrm{kA}$ & 1.350 & 1.350 & $\mathrm{x}$ & $\mathrm{x}$ & $\mathrm{kA}$ & $\mathrm{kA}$ & $\mathrm{x}$ & $\mathrm{x}$ & $\mathrm{x}$ \\
\hline & $\begin{array}{l}\text { Wirtschaftspark Bruck } \\
\text { an der Mur }\end{array}$ & 1998 & 27 & 146 & 2.134 & 1.855 & $\mathrm{x}$ & $\mathrm{x}$ & $\mathrm{x}$ & $\mathrm{x}$ & & $\mathrm{x}$ & $\mathrm{x}$ \\
\hline
\end{tabular}




\begin{tabular}{|c|c|c|c|c|c|c|c|c|c|c|c|c|c|}
\hline Bundesland & Zentrum & $\begin{array}{l}\text { Grün- } \\
\text { dung }\end{array}$ & Firmen & $\begin{array}{c}\text { Arbeits- } \\
\text { plätze }\end{array}$ & \begin{tabular}{c|}
$\begin{array}{c}\text { vermietbare } \\
\text { Fläche }\end{array}$ \\
\end{tabular} & \begin{tabular}{|c|} 
davon \\
Bürofläche
\end{tabular} & $\begin{array}{l}\text { Gründer- } \\
\text { beratung }\end{array}$ & $\begin{array}{l}\text { Geschäfts- } \\
\text { pläne }\end{array}$ & $\begin{array}{c}\text { Personal- } \\
\text { suche }\end{array}$ & $\begin{array}{c}\text { Technologie- } \\
\text { beratung }\end{array}$ & $\begin{array}{c}\text { Kooperations- } \\
\text { vermittlung }\end{array}$ & \begin{tabular}{|c|}
$\begin{array}{c}\text { Finanzierungs- } \\
\text { beratung }\end{array}$ \\
\end{tabular} & $\begin{array}{c}\text { Organisations- } \\
\text { beratung }\end{array}$ \\
\hline & \begin{tabular}{|l|} 
Wirtschaftspark \\
Kleinregion Fehring \\
\end{tabular} & 1999 & 18 & $\mathrm{kA}$ & 2.200 & 1.000 & $\mathrm{kA}$ & $\mathrm{kA}$ & $\mathrm{kA}$ & $\mathrm{kA}$ & $\mathrm{kA}$ & $\mathrm{kA}$ & $\mathrm{kA}$ \\
\hline & $\begin{array}{l}\text { Wirtschaftspark und } \\
\text { Gründerzentrum } \\
\text { Mürzzuschlag } \\
\end{array}$ & 1996 & 20 & 500 & 9.500 & 1.500 & $\mathrm{x}$ & $\mathrm{x}$ & $\mathrm{x}$ & $\mathrm{x}$ & $\mathrm{x}$ & $\mathrm{x}$ & $\mathrm{x}$ \\
\hline \multirow[t]{8}{*}{ Tirol } & $\begin{array}{l}\text { Competence Center } \\
\text { Innsbruck }\end{array}$ & 2003 & 15 & 15 & 3.940 & 3.750 & $\mathrm{x}$ & $\mathrm{kA}$ & $\mathrm{kA}$ & $\mathrm{kA}$ & $\mathrm{x}$ & $\mathrm{x}$ & $\mathrm{x}$ \\
\hline & $\begin{array}{l}\text { Gründer- und } \\
\text { Businesscenter Kufstein }\end{array}$ & 2003 & 49 & 150 & 6.200 & 6.000 & $\mathrm{x}$ & $\mathrm{x}$ & $\mathrm{x}$ & $\mathrm{x}$ & $\mathrm{x}$ & $\mathrm{x}$ & $\mathrm{x}$ \\
\hline & $\begin{array}{l}\text { Innovationszentrum } \\
\text { Landeck }\end{array}$ & $\mathrm{kA}$ & 26 & $\mathrm{kA}$ & $\mathrm{kA}$ & $\mathrm{kA}$ & $\mathrm{x}$ & $\mathrm{x}$ & $\mathrm{x}$ & $\mathrm{x}$ & $\mathrm{x}$ & $\mathrm{x}$ & $\mathrm{kA}$ \\
\hline & $\begin{array}{l}\text { Innovationszentrum } \\
\text { Reutte }\end{array}$ & 2000 & 24 & 75 & 2.025 & 1.900 & $\mathrm{x}$ & $\mathrm{x}$ & $\mathrm{x}$ & $\mathrm{x}$ & $\mathrm{x}$ & $\mathrm{x}$ & $\mathrm{x}$ \\
\hline & Life Science Center & $\mathrm{kA}$ & 9 & kA & $\mathrm{kA}$ & $\mathrm{kA}$ & $\mathrm{kA}$ & $\mathrm{kA}$ & $\mathrm{kA}$ & $\mathrm{kA}$ & $\mathrm{x}$ & $\mathrm{kA}$ & $\mathrm{kA}$ \\
\hline & $\begin{array}{l}\text { Osttiroler } \\
\text { Wirtschaftspark }\end{array}$ & 1997 & 27 & $\mathrm{kA}$ & 3.160 & 3.160 & $\mathrm{x}$ & $\mathrm{x}$ & $\mathrm{x}$ & $\mathrm{x}$ & $\mathrm{x}$ & $\mathrm{x}$ & $\mathrm{x}$ \\
\hline & $\begin{array}{l}\text { Technologie- und } \\
\text { Gründerzentrum } \\
\text { Hochfilzen }\end{array}$ & 1998 & 7 & 40 & 750 & 600 & $\mathrm{x}$ & $\mathrm{x}$ & (x) & $\mathrm{x}$ & $\mathrm{x}$ & $\mathrm{x}$ & $\mathrm{x}$ \\
\hline & $\begin{array}{l}\text { Technologie- und } \\
\text { Wirtschaftspark } \\
\text { Innsbruck }\end{array}$ & 1991 & 190 & 1.800 & 55.000 & 53.000 & $\mathrm{x}$ & $\mathrm{x}$ & $\mathrm{kA}$ & $\mathrm{x}$ & $\mathrm{x}$ & $\mathrm{x}$ & $\mathrm{x}$ \\
\hline \multirow[t]{9}{*}{ Vorarlberg } & $\begin{array}{l}\text { at \& co Regionales } \\
\text { Zentrum Ems }\end{array}$ & 2001 & 13 & $\mathrm{kA}$ & 2.230 & $\mathrm{kA}$ & $\mathrm{x}$ & $\mathrm{x}$ & $\mathrm{x}$ & $\mathrm{x}$ & $\mathrm{x}$ & $\mathrm{x}$ & $\mathrm{x}$ \\
\hline & $\begin{array}{l}\text { Competencecenter CCD } \\
\text { Dornbirn }\end{array}$ & 1996 & 9 & $\mathrm{kA}$ & 2.500 & $\mathrm{kA}$ & $\mathrm{x}$ & $\mathrm{x}$ & $\mathrm{x}$ & $\mathrm{x}$ & $\mathrm{x}$ & $\mathrm{x}$ & $\mathrm{x}$ \\
\hline & $\begin{array}{l}\text { Competence Center } \\
\text { Rheintal }\end{array}$ & 2002 & 27 & $\mathrm{kA}$ & 3.116 & $\mathrm{kA}$ & $\mathrm{x}$ & $\mathrm{x}$ & $\mathrm{x}$ & $\mathrm{x}$ & $\mathrm{x}$ & $\mathrm{x}$ & $\mathrm{x}$ \\
\hline & Impulszentrum Fabrik & 1997 & 19 & $\mathrm{kA}$ & 3.000 & $\mathrm{kA}$ & $\mathrm{x}$ & $\mathrm{x}$ & $\mathrm{x}$ & $\mathrm{x}$ & $\mathrm{x}$ & $\mathrm{x}$ & $\mathrm{x}$ \\
\hline & \begin{tabular}{|l|} 
Impulszentrum \\
Bregenzerwald
\end{tabular} & 2001 & 18 & $\mathrm{kA}$ & 2.200 & $\mathrm{kA}$ & $\mathrm{x}$ & $\mathrm{x}$ & $\mathrm{x}$ & $\mathrm{x}$ & $\mathrm{x}$ & $\mathrm{x}$ & $\mathrm{x}$ \\
\hline & $\begin{array}{l}\text { Innovationszentrum } \\
\text { Bludenz }\end{array}$ & 2001 & 7 & $\mathrm{kA}$ & 772 & $\mathrm{kA}$ & $\mathrm{x}$ & $\mathrm{x}$ & $\mathrm{x}$ & $\mathrm{x}$ & $\mathrm{x}$ & $\mathrm{x}$ & $\mathrm{x}$ \\
\hline & \begin{tabular}{|l|} 
Interpark Focus \\
\end{tabular} & $\mathrm{kA}$ & 9 & kA & 120.000 & $\mathrm{kA}$ & $\mathrm{x}$ & $\mathrm{x}$ & $\mathrm{x}$ & $\mathrm{x}$ & $\mathrm{x}$ & $\mathrm{x}$ & $\mathrm{x}$ \\
\hline & $\begin{array}{l}\text { Unternehmerzentrum } \\
\text { Alberschwende }\end{array}$ & 2005 & 3 & $\mathrm{kA}$ & $\mathrm{kA}$ & $\mathrm{kA}$ & $\mathrm{x}$ & $\mathrm{x}$ & $\mathrm{x}$ & $\mathrm{x}$ & $\mathrm{x}$ & $\mathrm{x}$ & $\mathrm{x}$ \\
\hline & \begin{tabular}{|l|} 
icub \\
\end{tabular} & 2005 & 6 & & & & & & & & & & \\
\hline Wien & $\begin{array}{l}\text { Business- und Research } \\
\text { Center Höchstädtplatz } \\
\text { (in Gründung) }\end{array}$ & 2005 & & & 3.400 & 3.200 & $\mathrm{x}$ & $\mathrm{x}$ & $\mathrm{kA}$ & $\mathrm{x}$ & $\mathrm{x}$ & $\mathrm{x}$ & $\mathrm{x}$ \\
\hline
\end{tabular}




\begin{tabular}{|c|c|c|c|c|c|c|c|c|c|c|c|c|c|}
\hline Bundesland & Zentrum & $\begin{array}{l}\text { Grün- } \\
\text { dung }\end{array}$ & Firmen & $\begin{array}{c}\begin{array}{c}\text { Arbeits- } \\
\text { plätze }\end{array} \\
\end{array}$ & $\begin{array}{c}\text { vermietbare } \\
\text { Fläche }\end{array}$ & \begin{tabular}{|c|} 
davon \\
Bürofläche
\end{tabular} & $\begin{array}{l}\text { Gründer- } \\
\text { beratung }\end{array}$ & $\begin{array}{l}\text { Geschäfts- } \\
\text { pläne }\end{array}$ & $\begin{array}{c}\text { Personal- } \\
\text { suche }\end{array}$ & $\begin{array}{l}\text { Technologie- } \\
\text { beratung }\end{array}$ & $\begin{array}{c}\text { Kooperations- } \\
\text { vermittlung }\end{array}$ & $\begin{array}{c}\begin{array}{c}\text { Finanzierungs- } \\
\text { beratung }\end{array} \\
\end{array}$ & $\begin{array}{l}\text { Organisations- } \\
\text { beratung }\end{array}$ \\
\hline & $\begin{array}{l}\text { Competence Center } \\
\text { Wien }\end{array}$ & 2004 & 10 & $\mathrm{kA}$ & 4.850 & $\mathrm{kA}$ & $\mathrm{kA}$ & $\mathrm{kA}$ & $\mathrm{kA}$ & $\mathrm{kA}$ & $\mathrm{x}$ & $\mathrm{kA}$ & $\mathrm{kA}$ \\
\hline & Impulszentrum IP.ONE & 2001 & 11 & 80 & 6.800 & $\mathrm{kA}$ & $\mathrm{kA}$ & $\mathrm{kA}$ & $\mathrm{kA}$ & $\mathrm{kA}$ & $\mathrm{x}$ & $\mathrm{kA}$ & $\mathrm{kA}$ \\
\hline & Impulszentrum IP.TWO & 2003 & 11 & 50 & 4.300 & $\mathrm{kA}$ & $\mathrm{kA}$ & $\mathrm{kA}$ & $\mathrm{kA}$ & $\mathrm{kA}$ & $\mathrm{x}$ & $\mathrm{kA}$ & $\mathrm{kA}$ \\
\hline & $\begin{array}{l}\text { Technologie- und } \\
\text { Gewerbezentrum SIG } \\
\text { Wien }\end{array}$ & 1989 & 72 & $\mathrm{kA}$ & 5.338 & 4.000 & $\mathrm{x}$ & $\mathrm{x}$ & $\mathrm{kA}$ & $\mathrm{x}$ & $\mathrm{x}$ & $\mathrm{x}$ & $\mathrm{x}$ \\
\hline & Vienna Bio Center & 2004 & 4 & $\mathrm{kA}$ & 4.200 & $\mathrm{kA}$ & $\mathrm{kA}$ & $\mathrm{kA}$ & $\mathrm{kA}$ & $\mathrm{kA}$ & $\mathrm{x}$ & $\mathrm{kA}$ & $\mathrm{kA}$ \\
\hline & Tech Gate Vienna & 2002 & 30 & $\mathrm{kA}$ & $\mathrm{kA}$ & $\mathrm{kA}$ & $\mathrm{kA}$ & $\mathrm{kA}$ & $\mathrm{kA}$ & $\mathrm{kA}$ & $\mathrm{kA}$ & $\mathrm{kA}$ & $\mathrm{kA}$ \\
\hline
\end{tabular}

Quelle: Verband der Technologiezentren Österreich, www.vto.at, Homepages der diversen Zentren 


\section{$6 \quad$ Welche Rolle spielt die Entwicklungsagentur des Landes?}

Wir haben an verschiedenen Stellen die Rolle der Entwicklungsagentur des Landes bzw. die Landesregierungen angesprochen. In ihrer Minimalrolle kommen sie für die Ko-Finanzierung auf. In vielen Fällen geht ihre Rolle, zumal jene der Entwicklungsagenturen, mitunter deutlich darüber hinaus. Gleichzeitig variiert die aktuelle Bedeutung erheblich, selbst die vermeintlich klare Rolle der Ko-Finanzierung und damit verbunden, die Abgabe einer entsprechenden Zusage. Wir haben im Einzelnen folgendes Repertoire an Rollen und Verhaltensweisen gefunden:

\section{Zentrale Koordination der Impulszentren}

Prototyp ist hier die Oberösterreichische Technologie Marketing Gesellschaft (TMG). Sie ist in der Regel an den einzelnen Impulszentren eigentumsmäßig beteiligt. Auf den ersten Blick sollte das eine sowohl notwendige wie hinreichende Bedingung für eine enge Abstimmung der Politik des Landes, hier vertreten durch die TMG ${ }^{59}$, und den Impulszentren bedeuten. Wir vertreten indes eine Position, die dem Eigentum weder eine notwendige noch eine hinreichende Bedingung für enge Politikkoordination zuschreibt. Ein Beispiel für eine außerordentlich starke Beziehung sowohl zwischen Landesregierung als auch Entwicklungsagentur ist das Land Niederösterreich, die zusammen als Eigentümer der RIZ Holding fungieren, aber bei Weitem nicht die enge Politikkoordination aufweist wie in Oberösterreich.

Aber wir bestreiten nicht, dass Eigentum die Beziehung begünstigt, auch wenn es sich nur um Minderheitenbeteiligungen handelt. Vollständig wird das Bild der oberösterreichischen Szene durch ein paar zusätzliche Ingredienzien. Zunächst gibt es dort ein ziemlich strenges Berichtswesen und ein damit verbundenen regelmäßiges Feedback zwischen den Impulszentren und der TMG. Ferner gibt es einen hohen Grad an Kontinuität bei den handelnden Personen seitens der TMG. Transportmittel dieser engen Beziehung ist nicht nur Eigentum und Berichtswesen, sondern auch die Existenz eines insgesamt betrachtet nicht allzu großen Projektes seitens der Landesregierung (Größenordnung: EUR 500.000), welches eine Reihe von 'Clubgütern' ermöglicht hat: Aus- und Weiterbildung, Einführung von Standards, Vorbereitung von gemeinsamen Initiativen usw..

Nicht unerwähnt soll in diesem Zusammenhang die Durchführung eines RIFupgrade Projekts namens Qnet, welches das Ziel verfolgt hat, durch Einführung gemeinsamer Qualitätsstandards die Leistungsfähigkeit und Effizient der Zentren zu heben. Im Rahmen dieses Projekts wurden einheitliche Mietverträge entwickelt, gemeinsamer Einkauf von Versicherungs- und Qualifizierungsleistungen organisiert, Erfahrungsgruppen eingerichtet ${ }^{60}$, Kennziffern entwickelt, Benchmarks durchgeführt, ein Qualitätshandbuch erstellt. Schließlich dienen diese Maßnahmen nicht nur zum Abgleich der einzelnen Zentren sowie zur Identifikation und Verbreitung von überlegenen Konzepten, sondern auch zur raschen Einarbeitung neuer (leitender) Mitarbeiter.

59 Dies ist tatsächlich der Fall, als zwischen TMG und Amt der Landesregierung bzw. Landesregierung eine sehr enge und lückenlose Abstimmung vorliegt.

60 Zu vier Themen - Qualitätsmanagement, Marketing, Innovation, Gründung -, mit einer Tagungsfrequenz von einem Monat. 
Bei aller Wertschätzung der Vorbildhaftigkeit der durchgeführten Aufgaben können wir uns dem Eindruck nicht verschließen, dass die Finanzierung dieses Qnet-Projekts auch durch das Land hätte erfolgen können, was ja in Ansätzen auch schon der Fall gewesen ist - ein politischer Mitnahmeeffekt also, weil hier das Prinzip der Subsidiarität nicht sehr ernst genommen wurde. Es ist, was die Sache selbst betrifft, das Projekt Qnet inzwischen auf die Bundesebene aufgestiegen und wird dort akklamiert, insofern innerhalb des Verbandes der Technologiezentren Österreichs (VTO) das Thema Qualität und Qualitätsmanagement von den bei Qnet maßgeblichen Personen systematisch wahrgenommen wird. Weil Qnet an den Bund im Rahmen von RIFupgrade zur Förderung herangetragen wurde und das zuständige bmvit eine Ko-Finanzierung zugesagt hat, wurde im Fördervertrag richtigerweise vereinbart, es auch anderen Bundesländern zur Verfügung zu stellen. Diese Auflage wird nun zweckmäßigerweise vom VTO koordiniert und so die Ergebnisse des Qnet umgesetzt.

Die TMG nimmt auch in Bezug auf die RIF 2000 Projekte im Allgemeinen und die REGplus Projekte im Besonderen eine besondere Funktion ein. Ihre Aufgabe besteht darin, frühzeitig Informationen über die Möglichkeit einer Förderung unter den potenziellen Antragstellern zu verbreiten, die Koordination der Anträge zu übernehmen, und das Einvernehmen mit dem Land über die Ko-Finanzierung herzustellen.

Auch wenn einzelne Zentrumsmanager die Unterstützung durch die TMG in ihrem konkreten Fall herunterspielen ${ }^{61}$, macht sich die Zentralisierung der Betreuung der Impulszentren nicht zuletzt in der sehr konstanten und zugleich hohen Erfolgsquote bei den oberösterreichischen Beteiligungen bemerkbar. Es fällt nicht schwer, dem oberösterreichischen Vorgehen das Attribut einer guten Praxis zuzuschreiben.

\section{Informelle Einbindung und permanenter Dialog}

Hier ist die Steiermark als Prototyp zu erwähnen. Nicht, dass dies nicht auch in anderen Bundesländern zutrifft, in der Steiermark zeigt sich der Erfolg einer hohen Beziehungsintensität zwischen dem Land (hier: Steirische Wirtschaftsförderungs Gesellschaft, SFG) und den Zentren in ganz besonderem Ausmaß. Spezifisch ist hier, dass die SFG, repräsentiert durch ihre Tochter, Innofinanz, gelegentlich auch selbst als Antragsteller und Förderempfänger aufgetreten ist. Die Innofinanz selbst ist Miteigentümer einer Reihe von Impulszentren in der Steiermark und übt über diese Funktion neben der formalen Aufsichtsrolle auch eine eher informelle, aber nichtsdestoweniger wirksame Koordinationsfunktion aus. Gleichzeitig tritt die SFG als Ko-Finanzier auf $^{62}$.

Insgesamt ist die Landschaft der Impulszentren in der Steiermark wesentlich bunter als anderswo. Dies ist gezielte Politik und hat auch damit zu tun, dass das Land (SFG) durch frühe Involvierung in neue Ideen und Vorhaben diese durch gemeinsames Weiterdenken und Weiterentwickeln belohnt. Nicht ganz zufällig, aber nicht zwangsläufig drückt sich dieses gemeinsame Entwickeln von Ideen in gemeinsamem Umsetzen, also in Kooperation aus. Gleichwohl ist zu bemerken, dass dies nicht durchgängig stattfindet.

\footnotetext{
61 Nicht ganz zufällig gibt es in Oberösterreich einige 'Förderprofis'.

62 Dass hier komplizierte, ja delikate Governance- und Kontrollprobleme auftauchen, sei hier nur angedeutet und ist vor allem eine Aufgabe, die von der Steirermark selbst zu lösen ist.
} 
Sieht man von zwei, nicht unwesentlichen Problemen ab, nämlich die delikaten Governance- und Kontrollverhältnisse und die mitunter überdimensionierte Zahl an gemeinschaftlichen Projekten, verdient das steirische Verhalten durchaus auch Attribut 'gute Praxis'.

\section{Verstreute Beobachtungen}

Beginnen wir mit einem Einzelfall, der wert ist, beachtet zu werden. Es ist dies Vorarlberg, wo das Land Vorarlberg im Rahmen seiner Innovations- bzw. Wirtschaftspolitik Inkubatoren vorgesehen, gleichzeitig aber den Wunsch nach möglichst großem privatem Engagement geäußert hat. Dies hat in der Folge zu einem erheblichen Engagement einer Gruppe privater Unternehmen geführt, wobei die PRISMA Holding durchgängig präsent ist. Obwohl es Gründe gibt, die ein Engagement der öffentlichen Hand wünschenswert erscheinen lassen ${ }^{63}$, sollte dort, wo privates Engagement möglich ist, dieses bevorzugt werden. Vorarlberg zeigt grosso modo, dass dies möglich ist. In diesem Sinn ist Vorarlberg ein nicht uninteressantes Benchmark, an dem man immer wieder Maß nehmen sollte.

In Tirol wurde auf Ebene der Landesregierung bzw. der Entwicklungsagentur (hier: Tiroler Zukunftsstiftung) in den vergangen Jahren ein Prozess der Konsolidierung eingeleitet und weitgehend abgeschlossen. Zeitgleich mit diesem Konsolidierungsprozess, teils durch die nunmehr konsolidierten Strukturen begünstigt, teils als von außen kommend (namentlich durch diverse Bundesprogramme wie $\mathrm{K}_{\text {ind/net }}$ und AplusB), hat sich in Tirol eine neue Spezies von Trägern bzw. Förderempfängern entwickelt. Die beiden REGplus Projekte BIO.COM.NET und BIO.COM.NET 2 werden nicht von klassischen Impulszentren durchgeführt, sondern zunächst von akademischen Inkubatoren aus dem AplusB Programm und dann von Forschungseinrichtungen aus dem $K_{\text {ind/net }}$ Programm ${ }^{64}$. Tirol ist insgesamt ein gutes Beispiel für eine Zusammenfassung der Koordination verschiedener innovationsorientierter Aktivitäten (Impulszentren, AplusB, Kompetenzzentren, Cluster etc.) in einer zentralen Institution (hier: Tiroler Zukunftsstiftung).

Niederösterreich ist noch ein erwähnenswerter Fall. Hier gibt es im Zusammenhang mit Impulszentren die alles überragende RIZ-Holding. Hier gibt es Gebietsaufteilungen und mit vier Untergesellschaften. Während die RIZ-Holding zu 55\% dem Land Niederösterreich und zu 45\% der ecoplus gehört, befinden sich die vier Untergesellschaften jeweils im Mehrheitseigentum (51\%) der RIZ-Holding sowie einzelnen Städten, Gemeinden bzw. Trägervereinen. Interessanter Weise gibt es neben der

63 Diese sind weniger finanzieller als vielmehr die symbolischer Natur, weil über die Beteiligung etwa von mehreren benachbarten Gemeinden ein höherer Identifikationsgrad der lokalen Institutionen und politischen Entscheidungsträger mit der lokalen Wirtschaft erhöht werden kann.

64 Genau genommen ist die Bezeichnung 'Forschungseinrichtung' im Grunde irreführend, insofern als hier keine klassischen Unternehmensfunktionen ausgeübt werden wie Allokation von Budgetmitteln, Disposition von Anlagen und Personal, Übernahme und Management von Risiken, Management von Cash-Flow, Verwertung von Ergebnissen usw.. Vielmehr werden Fördermittel administriert, eine Aufgabe, die auch von einer professionell arbeitenden Förderagentur übernommen werden könnte. Freie Personalkapazität hat hier dazu geführt, dass zusätzliche Arbeit gesucht - und gefunden wurde. So sehr die pulsierende Entwicklung in Tirol zu begrüßen ist, so sehr stellt sich die Frage, ob es zweckmäßig ist, die Entwicklung zusätzlicher öffentlicher intermediärer Strukturen zu begünstigen. 
RIZ-Gruppe auch noch mehrere Zentren, die sich im (mehrheitlichen) Eigentum der ecoplus befinden. Neben diesen soweit von der öffentlichen Hand betriebenen Zentren gibt es auch noch einige in privatem Eigentum. Nicht zuletzt hat sich im Zuge des REGplus Programms gezeigt, dass sich gerade in Niederösterreich zahlreiche virtuelle Zentren bebildet haben. Insgesamt war die niederösterreichische Beteiligung von einer auffallend geringen Erfolgsquote geprägt. Wir erwähnen den Fall Niederösterreich, weil hier ein reiches ${ }^{65}$, auch traditionsreiches Bundesland - die Gründung des RIZ Niederösterreich Süd in Wr. Neustadt fand 1988 statt - einer eher schwach entwickelten Governance gegenübersteht. Diese ist vor allem durch eine schwache Anbindung an die beiden Eigentümer und dieser mit der RIZ-Holding gekennzeichnet. Dies führt dazu, dass außerhalb der RIZ-Holding andere Zentren, ebenfalls mit öffentlichem Geld, errichtet wurden, das jüngste liegt erst ein paar Jahre zurück. In der Folge fehlt es an Kooperation und Koordination, zuletzt darin, dass das Land Niederösterreich der RIZ-Holding die Ko-Finanzierung der REGplus-Projekte a priori verweigert hat ${ }^{66}$.

\section{Schlüsselfaktoren beim Umgang mit und über die Rolle der Landeseinrichtungen}

Gibt es optimale Rollenaufteilungen und Beziehungsformen zwischen den Impulszentren und den Einrichtungen der Länder, um das Angebot der RIF 2000 Förderung effizient zu nützen? Manche sagen, Eigentum und eigentumsrechtlicher $\mathrm{Zu}$ - und Durchgriff seien der Schlüsselfaktor. Als Beispiel werden hier Oberösterreich und die Steiermark genannt. Leider liefert ein in dieser Hinsicht gut aufgestelltes Bundesland wie Niederösterreich eine schwache Performance, wohingegen in Vorarlberg, das eine recht muntere Szene aufweisen kann, das Land eher im Hintergrund bleibt. Dieser Hinweis soll vorderhand genügen, um die Vorstellung einer wie immer gearteten best practice zu zerstreuen. Aber es lassen sich ungeachtet dessen einige hilfreiche Elemente von guter Praxis angeben.

- Kontinuität und Rollenklarheit bei der Entwicklungsagentur bzw. beim Land. Beispiele, wo dies gut funktioniert, sind Oberösterreich und die Steiermark, auch Vorarlberg. Diskontinuitäten und unklare Rollen sind u.a. in Kärnten und Niederösterreich, in jüngerer Vergangenheit auch im Burgenland zu beobachten.

- Aktive Rolle der Entwicklungsagenturen bzw. Länder. Es schadet nicht, wenn es auf Ebene der Länder eine einigermaßen selektive Politik gibt. Diese ist allein über die Selektivität, aber auch aus anderen Gründen stets eine aktive Politik. Im gegenständlichen Zusammenhang unterstützt sie die Awareness, das Förderangebot überhaupt aufzugreifen und zu nutzen. Sie ist förderlich für die Qualität der Anträge und übt auch eine gewisse Vorab-Selektion der Projekte aus und sichert dadurch ein Mindestniveau an Kompatibilität mit der Politik des Landes.

- Institutionelles Gedächtnis und Lernen. Beide vorstehenden Prinzipien begünstigen institutionelles Gedächtnis und Lernen bzw. setzen dieses voraus. Sie wir-

65 Allein die RIZ-Holding mit ihren Untergesellschaften beschäftigt 50 Mitarbeiter bzw. 37 Vollzeitäquivalente.

66 Es gibt in dieser Verweigerung eine gewisse Logik: Immerhin sind unter dem Dach der RIZHolding 50 Mitarbeiter / 37 VZÄ beschäftigt, die insgesamt ca. 100 Unternehmen mit durchschnittlich 3 Mitarbeitern betreuen. So gesehen stellt sich die Frage, welchen marginalen Ertrag ein zusätzlicher Euro bringen sollte. 
ken also wechselseitig verstärkend. Die Folge ist eine gewisse Mitentwicklung der Projekte und ein (symbolisches) Miteigentum an den Projekten. In fortgeschritteneren Kontexten entwickelt sich über die Zeit ein Coaching-Verhältnis zwischen den Zentren und den Entwicklungsagenturen.

- Miteigentum und Not-Invented-Here-Syndrom. Wir haben in einzelnen Fällen feststellen können, dass die Einbindung der Landesorganisationen auch deshalb wichtig ist, um bestimmten vagabundierenden Ängsten und Irritationen vorzubeugen ${ }^{67}$. Hier sind Besuche der Programmverantwortlichen auf Seiten des Bundes bei den Ländern bzw. gewisse Vereinbarungen über Controlling und sonstige Verfahrensregeln ausgesprochen förderlich. Hier ist abermals Partnerschaft und das Coaching-Prinzip am Ende hilfreicher als eine zwar formal korrekte, aber insgesamt kommunikationsarme Beziehung. Alles, was dazu beiträgt, dass die Förderagentur des Bundes und die einschlägigen Stellen auf Landesseite die Projekte als 'ihre' Projekte betrachten, sollte gewürdigt und gefördert werden.

- Alles in Allem geht nichts über eine aufgeräumte und pro-aktive Landesentwicklungsagentur. Wenn diese die Option RIF 2000 aktiv bewirtschaftet, ist ein hoher Grad an Erfolgsaussicht gegeben: Frühe Information und Involvierung, Qualitätssicherung und Prioritätensetzung bzw. Einpassung in die Strategien des Landes (zusammen mit dem Land), Weiterentwicklung des Portfolios ${ }^{68}$.

67 Man nennt dieses Verhalten das Not-Invented-Here-Syndrom. "Abmachungen mit Wien" oder "Schon wieder so ein EU-Projekt, wo wir nur zur Kasse gebeten werden." sind beliebte Abwehrreaktionen, bei denen die - manchmal nicht unbegründete - Angst ausgedrückt wird, vor vollendete Tatsachen gestellt zu werden.

68 Dass dann, wie im Falle Oberösterreichs, Aufgaben, die vorher das Land finanziert hat, auf die Bundesebene verlagert, so schmerzt dies ein wenig. Dieser Schmerz wird in des dadurch gelindert, dass der Bund die Ko-Finanzierungszusage unter der Auflage erteilt hat, dass die Ergebnisse den anderen Bundesländern zur Verfügung gestellt werden müssen. 


\section{$7 \quad$ Woran kann man nach zwei, drei Jahren die Spuren des Projekts noch erkennen?}

So haben wir die Frage nach den Wirkungen gestellt. Obwohl die Frage immer das REGplus oder RIFupgrade Projekt als Bezugspunkt hatte, war es erstaunlich, wie oft die Gesprächspartner auf das Zentrum und ihr eigenes zurückliegendes Tun Bezug genommen haben. Wir registrieren dies mit einem weinenden und mit einem lachenden Auge. Das weinende Auge soll ausdrücken, dass der marginale Beitrag des jeweiligen Projekts für das Fortkommen des Zentrums nur ausnahmsweise rekonstruiert werden kann und dass dies im Lichte der insgesamt durchgeführten Projekte vgl. Kapitel 5.3 bzw. Tabelle 23 - tatsächlich schwer fällt. Das lachende Auge weist darauf hin, dass die Projektleiter - sie sind entweder mit wenigen Ausnahmen die Leiter der Zentren oder es gibt eigene Projektleiter, dann haben diese in der Regel ein Portfolio von Projekten zu managen - jeweils das Zentrum als Ganzes im Auge haben. Wir wollen daher im Folgenden versuchen, im Rahmen einer Typologie die bunte Welt der Wirkungen von REGplus bzw. RIFupgrade Projekten zu charakterisieren.

Tabelle 27 Wirkungen und ausgebliebene Wirkungen der RIF 2000 Projekte

\begin{tabular}{|c|c|}
\hline $\begin{array}{l}\text { Wirkung / ausgebliebene Wirkung } \\
\text { [teilweise in Form von Zitaten] }\end{array}$ & Kommentar \\
\hline $\begin{array}{l}\text { Die am Regplus Projekt teilnehmende Firma aus } \\
\text { dem Zentrum erhält für die Dauer des Projekts } \\
\text { Aufträge und daher eine gewisse Grundaus- } \\
\text { lastung. }\end{array}$ & $\begin{array}{l}\text { Wir klassifizieren dies im Wesentlichen als } \\
\text { Beschaffungsvorgang ohne nennenswerte } \\
\text { Additionalitätswirkung. }\end{array}$ \\
\hline $\begin{array}{l}\text { Die Schüler der benachbarten HTL haben die } \\
\text { Möglichkeit, im Labor, welches im Rahmen des } \\
\text { Projekts eingerichtet wurde, Übungen zu } \\
\text { absolvieren. Dies festigt auch die Beziehung zu } \\
\text { den Lehrern der HTL, die ihrerseits für Spezial- } \\
\text { fragen als Berater dienen, was sich in zwei } \\
\text { Fällen bereits praktisch realisiert hat. }\end{array}$ & $\begin{array}{l}\text { Lehrer von HTLs gehen oft zwei Berufen nach, was } \\
\text { in der Regel vorteilhaft ist. Die Nähe zu HTLs in } \\
\text { Verbindung mit einer flächendeckenden Regionali- } \\
\text { sierungsstrategie bei Impulszentren ist im Prinzip } \\
\text { eine gute Politik, soweit sich dies organisieren lässt. } \\
\text { Dummerweise sind im konkreten Fall Impuls- } \\
\text { zentrum und HTL } 2 \text { km entfernt, was umso betrüb- } \\
\text { licher ist, als in diesem Bundesland Errichtung von } \\
\text { HTLs und Errichtung von Impulszentren demselben } \\
\text { politischen Gedanken entspringen. }\end{array}$ \\
\hline $\begin{array}{l}\text { Hier am Land ist es halt sehr schwer, Unterneh- } \\
\text { mensgründung und -entwicklung zu unterstützen, } \\
\text { man hat doch einige Nachteile, wenn man nicht } \\
\text { im Zentrum nicht, obwohl es nur } 45 \text { min nach } \\
\text { [Landeshauptstadt] sind. Die Kunden unserer } \\
\text { Dienstleistungen sind doch mehr im Ballungs- } \\
\text { raum. Daher sind auch die Projekte mit der } \\
\text { Fachhochschule so wichtig. }\end{array}$ & $\begin{array}{l}\text { Die Nähe eines wirtschaftlichen Zentrums gerät } \\
\text { eher zum Nachteil denn zum Vorteil. Ein Zentrum } \\
\text { ist u.a. deswegen ein Zentrum, weil es für die } \\
\text { umliegende Region spezifische Ressourcen eben im } \\
\text { Zentrum konzentriert. Erst im Zuge so genannter } \\
\text { Suburbanisierungsprozesse profitiert wieder die } \\
\text { Peripherie. }\end{array}$ \\
\hline $\begin{array}{l}\text { Wir haben ERFA-Treffen durchgeführt. Dabei } \\
\text { hat jedes Unternehmen vorgetragen, teils über } \\
\text { scheinbar ganz langweilige Themen wie z.B. } \\
\text { Arbeitszeitmodelle. Dabei gab es unter den } \\
\text { Teilnehmern, aber auch bei uns jede Menge Aha- } \\
\text { Erlebnisse, so dass wir diese Aktion in Zukunft } \\
\text { fortsetzen wollen. }\end{array}$ & $\begin{array}{l}\text { Eine häufig vernachlässigte, aber umso wirkungs- } \\
\text { vollere Aufgabe. Ein Zeichen übrigens für die } \\
\text { Regionalentwicklungsfunktion von Impulszentren. }\end{array}$ \\
\hline
\end{tabular}




\begin{tabular}{|c|c|}
\hline $\begin{array}{l}\text { Wirkung / ausgebliebene Wirkung } \\
\text { [teilweise in Form von Zitaten] }\end{array}$ & Kommentar \\
\hline $\begin{array}{l}\text { Wir haben zahlreiche Schulungen absolviert, } \\
\text { deren Bedarf wir vorher sondiert haben. Diese } \\
\text { sind sehr gut angekommen. Dabei haben wir ein } \\
\text { recht beachtliches Netzwerk aufgebaut. }\end{array}$ & $\begin{array}{l}\text { Ausgezeichnet. Dieses Netzwerk gilt es nun zu } \\
\text { bewirtschaften. }\end{array}$ \\
\hline $\begin{array}{l}\text { Wir haben Fachveranstaltungen im [Fach- } \\
\text { bereich] durchgeführt. Alle (20) Teilnehmer } \\
\text { waren aus [Landeshauptstadt], auch von großen } \\
\text { Unternehmen. Aus der engeren oder weiteren } \\
\text { Umgebung von [Standort des Zentrums] war } \\
\text { weit und breit niemand zu sehen. }\end{array}$ & $\begin{array}{l}\text { Ein typischer Fall einer angebotsseitigen Dienst- } \\
\text { leistung. Vgl. den vorstehenden Punkt als die } \\
\text { klügere Alternative ('vorher sondiert'). }\end{array}$ \\
\hline $\begin{array}{l}\text { Wir haben ein Labor aufgebaut, das wir nun } \\
\text { kommerziell betreiben wollen. Wir führen } \\
\text { gegenwärtig die ersten Gespräche. In spätestens } \\
\text { drei Jahren soll dies kostendeckend laufen. }\end{array}$ & $\begin{array}{l}\text { Auch wenn es im Einzelfall glücken mag, halten } \\
\text { wir die Errichtung von Labors oder laborartigen } \\
\text { Infrastrukturen mit öffentlichen Mitteln aus vielen } \\
\text { Gründen für bedenklich. Sie sind in der Regel } \\
\text { insuläre Lösungen, die schlecht angebunden sind } \\
\text { und dann erst ihr Angebot suchen müssen. Wegen } \\
\text { der schlechten Anbindung und den geringen } \\
\text { Erlösen fehlen in der Folge die Mittel für Moderni- } \\
\text { sierung (eigene Methodenentwicklung, Erneuerung } \\
\text { der Wissensbasis, neue Geräte). So kann es passier- } \\
\text { en, dass sie sich nach } 5 \text { Jahren in 'Laborruinen' } \\
\text { verwandeln. Labors müssen entweder aus einem } \\
\text { Produktions- oder einem Forschungszusammen- } \\
\text { hang heraus entstehen und betrieben werden. } \\
\end{array}$ \\
\hline $\begin{array}{l}\text { Enttäuschend ist die geringe Bereitschaft der } \\
\text { KMU, die [Technologie] anzuwenden. Es hat } \\
\text { sich herausgestellt, dass die eigentlich einfachere } \\
\text { Konzepte brauchen. }\end{array}$ & $\begin{array}{l}\text { Ein typisches Schicksal eine angebotsseitigen und } \\
\text { wegen der Kleinheit meist sehr spezialisierten } \\
\text { Lösung. }\end{array}$ \\
\hline $\begin{array}{l}\text { Ich möchte mich eigentlich mehr aus der Projekt- } \\
\text { tätigkeit zurückziehen und stattdessen vermehrt } \\
\text { auf die Firmen zugehen und mehr Präsenz } \\
\text { zeigen, im Besonderen möchte ich Geschäfts- } \\
\text { konzepte entwickeln und begleiten können. Ich } \\
\text { möchte mehr Zeit haben, um meinen Mitarbei- } \\
\text { tern meine Erfahrung und meine Kontakte } \\
\text { weiterzugeben. }\end{array}$ & $\begin{array}{l}\text { Diese beiden Fälle sind nicht untypisch und weisen } \\
\text { auf die Substitution der regulären Arbeit - weit } \\
\text { umschrieben mit 'Kümmern um die Unternehmen } \\
\text { bzw. die Mitarbeiter' - durch Projektarbeit hin. Mit } \\
\text { einem sehr kritischen Auge betrachtet liegt hier der } \\
\text { Verdacht auf eine erhebliche Substitutionswirkung } \\
\text { nahe: "Ich habe keine Zeit für die Unternehmen, } \\
\text { weil ich mein Förderprojekt vorantreiben muss!" }\end{array}$ \\
\hline $\begin{array}{l}\text { Ich bräuchte eine zusätzliche Halbtagskraft. Aber } \\
\text { ich muss fürchten, dass mir die Gesellschafter } \\
\text { dies nicht genehmigen werden. }\end{array}$ & $\begin{array}{l}\text { Wir haben auf diese Ambivalenz der Geschäfts- } \\
\text { modelle - Regionalentwicklung vs. EGT und } \\
\text { Auslastung - bereits hingewiesen und betrachten } \\
\text { dies als Beleg dafür, dass vielfach 'unter beengten } \\
\text { Verhältnissen' gearbeitet wird. }\end{array}$ \\
\hline $\begin{array}{l}\text { Worauf ich am Meisten stolz bin, ist, dass ich } \\
\text { positiv bilanziere. }\end{array}$ & $\begin{array}{l}\text { Im konkreten Fall hilft das REGplus mit, ein } \\
\text { positives Betriebsergebnis zu erzielen. Ein anderer } \\
\text { Fall einer inkonsistenten Governance. } \\
\end{array}$ \\
\hline $\begin{array}{l}\text { Dann wird es einen Inkubator geben, der Bestand } \\
\text { haben wird. Wir werden uns auf Projekte } \\
\text { konzentrieren, die finanzierbar sind und dann die } \\
\text { Finanzierung aufstellen und betreuen. Wir } \\
\text { nutzen aber auch die Ressourcen von AplusB. }\end{array}$ & Ein Schelm, wer hier schlechtes denkt. \\
\hline
\end{tabular}




\begin{tabular}{|c|c|}
\hline $\begin{array}{l}\text { Wirkung / ausgebliebene Wirkung } \\
\text { [teilweise in Form von Zitaten] }\end{array}$ & Kommentar \\
\hline $\begin{array}{l}\text { Worüber ich gerne erzähle, ist folgendes Bei- } \\
\text { spiel: Zwei ehemalige technische Manager einer } \\
\text { ausländischen Firma legen ein zuerst schwaches } \\
\text { Konzept vor, dann haben wir doch angebissen } \\
\text { weil wir einen ziemlich guten Marketing-Mann } \\
\text { gefunden haben. Der USP war das technische } \\
\text { Know-how. } 700.000 \text { EUR Finanzbedarf. } 100.000 \\
\text { selber aufgestellt, je } 200.000 \text { von Inkubator, } \\
\text { Land und AWS. Inkubator macht den } \\
\text { Backbereich. Verkaufen weltweit, } 1 \text { Mio. EUR } \\
\text { Umsatz. Können sehr hohe Margen verlangen. } \\
\text { Das Ganze hat sich schon gelohnt. Andererseits } \\
\text { sind wir hinter Plan, weil wir gerne schon ein } \\
\text { paar Unternehmen mehr hätten. }\end{array}$ & Nur weiter so. \\
\hline $\begin{array}{l}\text { Das erste REGplus Projekt war ein Glücksfall: } \\
\text { Ich habe beim ersten Projekt } 50 \text { Unternehmen } \\
\text { kontaktiert. Dann kam das Projekt zustande und } \\
\text { unsere Akzeptanz stieg deutlich an. Jetzt kriege } \\
\text { ich bei den Unternehmen jederzeit einen Termin. } \\
\text { Außerdem haben wir pro Woche zwei Artikel in } \\
\text { der Presse. }\end{array}$ & $\begin{array}{l}\text { Auch dafür kann ein REGplus Projekt gut sein und } \\
\text { man sollte die symbolische Bedeutung von } \\
\text { Projekten nicht gering schätzen. Hier diente das } \\
\text { Projekt als 'Eintrittskarte'. }\end{array}$ \\
\hline $\begin{array}{l}\text { Aus den Informationen und Erfahrungen, sowie } \\
\text { aus den Kontakten, die wir im Zuge des Projekts } \\
\text { erarbeitet haben, können wir die nächsten 2-3 } \\
\text { Jahren leben. }\end{array}$ & $\begin{array}{l}\text { Mehr hat das Projekt nicht gebracht, aber auch nicht } \\
\text { weniger - sofern dieses Beziehungs- und Wissens- } \\
\text { kapital sorgfältig bewirtschaftet wird. }\end{array}$ \\
\hline $\begin{array}{l}\text { Einer der wichtigsten Erfolge des Projekts ist, } \\
\text { dass wir ein Folgeprojekt bekommen haben, so } \\
\text { dass es nun weitergeht. }\end{array}$ & $\begin{array}{l}\text { Hier erbrütet öffentliches Geld öffentliches Geld. } \\
\text { Eigentlich ein Alptraum für einen Förderer von } \\
\text { intermediären Organisationen. }\end{array}$ \\
\hline $\begin{array}{l}\text { Das wichtigste Ergebnis ist die Netzwerkbil- } \\
\text { dung, die hat es vorher nicht gegeben. Jetzt soll } \\
\text { eine eigene Gesellschaft gegründet werden um } \\
\text { die Nachhaltigkeit zu festigen. Zweck der neuen } \\
\text { Gesellschaft ist das Akquirieren von Projekten, } \\
\text { die dann von den Partnern abgearbeitet werden. }\end{array}$ & $\begin{array}{l}\text { Intermediäre Organisation gründen intermediäre } \\
\text { Organisationen - die Wiederkehr desselben } \\
\text { Alptraums. }\end{array}$ \\
\hline $\begin{array}{l}\text { Worauf ich aber am meisten stolz bin, sind } \\
\text { meine Mitarbeiter, die sowohl hoch motiviert } \\
\text { sind als auch die richtige Einstellung zu unserer } \\
\text { Aufgabe haben. }\end{array}$ & $\begin{array}{l}\text { Das sind keine schlechten Voraussetzungen, zumal } \\
\text { wir weiter oben gesehen haben, wie sensibel die } \\
\text { Schlüsselpersonen eines Zentrums für dessen Erfolg } \\
\text { sind. }\end{array}$ \\
\hline $\begin{array}{l}\text { Ich darf das gar nicht verraten, aber der größte } \\
\text { Effekt ist, dass ich soviel gelernt habe und dafür } \\
\text { auch noch bezahlt werden. Ich setze dieses } \\
\text { Wissen ein, um neue Ideen umzusetzen. Es gibt } \\
\text { so viel zu tun. }\end{array}$ & $\begin{array}{l}\text { So definiert sich ein tatsächlich ziemlich engagier- } \\
\text { ter und keineswegs erfolgloser Zentrumsmanager. } \\
\text { Nicht unsympathisch. }\end{array}$ \\
\hline $\begin{array}{l}\text { Es gibt uns erst seit 2001. Der eigentliche positi- } \\
\text { ve Effekt ist unsere Entwicklung im Allgemei- } \\
\text { nen. Der andere Geschäftsführer war sehr } \\
\text { wichtig, umtriebig. Jetzt gibt es interessante } \\
\text { Dienstleistungen und wir haben uns einen guten } \\
\text { Namen gemacht. }\end{array}$ & Ein guter Name kann vieles aufwiegen. \\
\hline $\begin{array}{l}\text { Enttäuschend ist, dass wir im Grunde keine } \\
\text { Gründungen zustande gebracht haben, obwohl } \\
\text { das eines unserer wichtigsten Ziele im REGplus } \\
\text { Projekt war. }\end{array}$ & $\begin{array}{l}\text { Hier hat auch das REGplus Projekt nicht über die } \\
\text { Tatsache geholfen, dass dieses Zentrum insgesamt } \\
\text { eine schwache Performance aufweist. Es ist } \\
\text { wichtig, zu verstehen, dass es nicht unwesentlich } \\
\text { um die Entwicklung von Personen geht. Kontext } \\
\text { und Geschichte mehr einbeziehen. }\end{array}$ \\
\hline
\end{tabular}




\begin{tabular}{|l|l|}
\multicolumn{1}{|c|}{$\begin{array}{c}\text { Wirkung / ausgebliebene Wirkung } \\
\text { [teilweise in Form von Zitaten] }\end{array}$} & \multicolumn{1}{c|}{ Kommentar } \\
\hline $\begin{array}{l}\text { Wir wollten 100 Arbeitsplätze schaffen. Dass wir } \\
\text { das nicht zustande gebracht haben, ist schon } \\
\text { enttäuschend. }\end{array}$ & $\begin{array}{l}\text { Wie konnte dieses Projekt mit dieser Zielsetzung } \\
\text { die Jury passieren? Vermutlich hatte dieses Projekt } \\
\text { einige andere Meriten. }\end{array}$ \\
\hline $\begin{array}{l}\text { Die Kooperation unter den Zentren ist noch nicht } \\
\text { voll entfaltet. Hier liegen wir hinter den }\end{array}$ & $\begin{array}{l}\text { Das mag schon recht und gut sein. Unserer } \\
\text { Einschätzung nach kooperieren die Zentren schon } \\
\text { Möglichkeiten. }\end{array}$ \\
& $\begin{array}{l}\text { zu viel und kümmern sich zu wenig um ihre } \\
\text { Kernaufgaben: Inkubator + Treibhaus. }\end{array}$ \\
\hline
\end{tabular}

Quelle: Interviews

Wir ersparen uns an dieser Stelle einen über die bereits abgegebenen Bemerkungen hinausgehenden Kommentar und tun dies im übernächsten Kapitel, bei dem es um die Zukunft des RIF 2000 Programms geht. 


\section{Lernen von Anderen: Eine Übersicht über die internationale Inkubatorszene}

Google ist immer eine gute Adresse für eine erste - weltweiche - Recherche. Dort zeigt sich, dass es Impulszentren gibt, allerdings nur in Österreich. Sucht man nach 'Technologiezentren' bzw. 'technology centers / centres', wird man fündig, erreicht aber eher spezialisierte Teilorganisationen von internationalen Unternehmen oder Universitäten und Forschungseinrichtungen. Erst bei 'incubators' wird man fündig. Der allergrößte Teil des Fundes betrifft die Präsentation von Inkubatoren, deren Dienstleistungen, deren Mieter usw.. Die Suche nach 'incubators benchmarking' führt schließlich zu den gewünschten Ergebnissen, darunter auch ein Sechser. Es ist die von der Europäischen Kommission, Generaldirektion Unternehmen beauftragte und 2002 fertig gestellte Benchmarking-Studie zu Inkubatoren ${ }^{69}$. Diese Vergleichsstudie wurde nicht zuletzt deswegen beauftragt, weil, obwohl zahlreiche Untersuchungen zum Thema vorliegen, eine vergleichende Analyse gefehlt hat. Wir werden im Folgenden von dieser Studien extensiven Gebrauch machen und die wichtigsten Ergebnisse darstellen. Der Sprachgebrauch bleibt österreichisch, also Impulszentren, obwohl eine Anpassung an internationale Gepflogenheiten angeraten wäre.

\subsection{Eigentümer, Finanziers, Investoren}

Dies ist eine der entscheidenden Fragen. Sie durchzieht im Grunde die gesamte Debatte, inwieweit nämlich Impulszentren hochwertige Immobilien sind oder eher einen regionalen Versorgungsauftrag haben. Letzteres wäre dann - weitgehend exklusiv - an die öffentliche Hand adressiert. Die Erfahrung zeigt, dass es wesentlich auf den Mix an Eigentümern, Finanziers und Investoren ankommt. Wir werden dieses Thema im Folgenden an mehreren wieder aufgreifen.

Es zeigt sich jedenfalls, dass Beiträge von privater Seite einerseits wünschenswert sind, andererseits diese aber mit Erträgen verbunden sind. Die Formen des Engagements und der Art der Rückzahlungen können aber erheblich variieren (z.B. Dividenden, Zugang zu Infrastruktur, Corporate venturing, Expertise). Es zeigt sich, dass in den frühen Phasen öffentliche Finanzierung entscheidend sein kann und dass es mehrere Jahre dauern kann, bis privates Kapital angezogen werden kann, sei es, um öffentliches Eigentum in privates zu überführen, sei es, dass die laufenden Kosten aus einschlägigen Dienstleistungen gedeckt werden. Tabelle 28 gibt eine Übersicht über Eigentümer bzw. Finanziers von Impulszentren. Sie zeigt, dass öffentliche Einrichtungen die Mehrheit darstellen, aber auch, dass in jedem fünften Fall private Einrichtungen eine Rolle spielen.

Quintessenz dieser vergleichenden Betrachtung ist, dass Österreich grosso modo demselben Muster folgt, was das Verhältnis öffentlich : privat betrifft. Eine Besonderheit in Österreich ist indes die überragende Rolle regionaler bzw. lokaler Schlüsselpartner. Grundsätzlich dürfen wir in dieser Hinsicht eine eher breite Palette von Dienstleistungen einerseits und eine eher breite Zielgruppe erwarten, was sich denn auch empirisch bestätigt (vgl. Kapitel 8.4).

69 European Commission, Enterprise Directorate-General, Benchmarking of Business Incubators, Final Report, Centre for Strategy \& Evaluation Services, February 2002 
Tabelle 28 Schlüsselpartner von Impulszentren

\begin{tabular}{|l|r|r|}
\hline \multicolumn{1}{|c|}{ Partner } & \multicolumn{1}{c|}{ Zahl } & \multicolumn{1}{c|}{ Anteil [\%] } \\
\hline (1) EU und / oder andere internationale Agenturen & 36 & 13,4 \\
\hline (2) National Einrichtungen / Agenturen & 68 & 25,3 \\
\hline (3) Unternehmen, Banken und andere private Organisationen & 56 & 20,8 \\
\hline (4) Universitäten / Forschungseinrichtungen & 44 & 16,4 \\
\hline (5) Kommunale Einrichtungen / NGO & 34 & 11,5 \\
\hline Gesamt & 269 & 100,0 \\
\hline
\end{tabular}

Quelle: European Commission, Enterprise Directorate-General, Benchmarking of Business Incubators, Final Report, Centre for Strategy \& Evaluation Services, February 2002

Interessant in diesem Zusammenhang ist die Frage, inwieweit Impulszentren erwerbsorientiert oder gemeinnützig sind. Die Antwort ist einerseits sehr deutlich, insofern eines aus fünf (21,8\%) erwerbsorientiert ist, wohingegen drei aus vier (76,9\%) gemeinnützig sind. Andererseits lässt sich beobachten, dass der Anteil an öffentlicher Finanzierung bzw. die gemeinnützige Orientierung von Land zu Land unterschiedlich hoch ist. Während in Italien 38,5\% der Zentren erwerbsorientiert sind, sind es in Frankreich lediglich 18\%.

\subsection{Standorte und Größe von Impulszentren}

Hier zeigt sich in einer internationalen Betrachtung ein wesentlicher Unterschied zu Österreich. Während im internationalen Vergleich Impulszentren nur im Ausmaß von 6,4\% ländliche Standorten aufweisen (vgl. Tabelle 29), ist dies in Österreich deutlich anders. Ein wesentlicher Erklärungsfaktor ist hier die dominante Rolle von regionalen und lokalen Partnern bzw. Eigentümern.

Tabelle 29 Standorte von Impulszentren

\begin{tabular}{|l|r|r|}
\hline & \multicolumn{1}{c|}{ Zahl } & \multicolumn{1}{c|}{ Anteil [\%] } \\
\hline Urbanes Umfeld & 68 & 54,4 \\
\hline Greenfield & 30 & 24,0 \\
\hline Ländliches Umfeld & 8 & 6,4 \\
\hline Andere & 13 & 10,4 \\
\hline k.A. & 6 & 4,8 \\
\hline Gesamt & $\mathbf{1 2 5}$ & $\mathbf{1 0 0 , 0}$ \\
\hline
\end{tabular}

Quelle: European Commission, op. cit.

Ein interessantes, und mit österreichischen Verhältnissen durchaus vergleichbares Phänomen betrifft die Art des Gebäudes: nur eines von fünf Gebäuden ist eine umgebaute Immobilie, wohingegen drei von fünf neu errichtet sind. Ihre Größe reicht von 90 bis $41.000 \mathrm{~m}^{2}$, der Median beträgt $3.000 \mathrm{~m}^{2}$, die eine Hälfte ist also größer, die andere kleiner als $3.000 \mathrm{~m}^{2}$. Die österreichischen Impulszentren sind - bis auf's Komma - im Median identisch gleich groß (vgl. Tabelle 26).

Tabelle 30 gibt eine Übersicht über die Zahl der betreuten Unternehmen / Organisationen. Im Median sind es 18 Mieter und 10 externe Einheiten. Ein Blick auf Abbildung 2 zeigt, dass die Verhältnisse in Österreich nicht wesentlich von denen anderswo abweichen. 
Tabelle 30 Zahl der betreuten Unternehmen / Organisationen

\begin{tabular}{|l|r|r|}
\hline \multicolumn{1}{|c|}{ Zahl der betreuten Einheiten } & \multicolumn{1}{c|}{ Mieter } & \multicolumn{1}{c|}{ Externe } \\
\hline Minimum & 1 & 1 \\
\hline Maximum & 120 & 571 \\
\hline Durchschnitt & 32.2 & 36.6 \\
\hline Median & 18 & 10 \\
\hline Median - Mieter und Externe & \multicolumn{2}{|c|}{28} \\
\hline
\end{tabular}

Quelle: European Commission, op. cit.

\subsection{Ziele, Rollen und strategischer Kontext von Impulszentren}

Hier schlägt ein durchaus gängiges Motiv durch: Wettbewerbsfähigkeit und Schaffung von Arbeitsplätzen. Damit sind Impulszentren nichts weiter als ein spezielles Instrument in einer breiteren Palette von Bemühungen, zwei zentralen Herausforderungen der Wirtschaftspolitik zu begegnen. Wichtiger als die individuellen Ziele der Zentren ist damit ihre Einbettung in weiter reichende Strategien und Politikmaßnahmen. In diesem Punkt befinden sich die meisten österreichischen Zentren in einer bevorzugten Position, die auch in der hier zugrunde liegenden Benchmark-Studie ausdrücklich als Best Practice Example erwähnt wird. Standalone-Betrieb ist daher die zweitbeste Lösung und die Einbettung in weiteres Netzwerk von Dienstleistungsanbietern und Geschäftspartnern die bevorzugenswertere Variante.

Tabelle $31 \quad$ Ziele von Impulszentren

\begin{tabular}{|l|r|r|r|r|r|r|r|}
\hline \multicolumn{1}{|c|}{ Ziele } & \multicolumn{6}{|c|}{ Rang (1 = am Wichtigsten) } \\
\cline { 2 - 8 } & 1 & 2 & 3 & 4 & 5 & 0 & Durchschnitt \\
\hline (1) Wettbewerbsfähigkeit und Schaffung von Arbeitsplätzen & 56 & 14 & 4 & 0 & 1 & 3 & 1,3 \\
\hline $\begin{array}{l}\text { (2) Unterstützung von Forschungseinrichtungen bei der } \\
\text { Kommerzialisierung ihres Know-how }\end{array}$ & 10 & 18 & 19 & 18 & 3 & 9 & \\
\hline (3) Unterstützung von Unternehmen bei Spin-off Aktivitäten & 3 & 23 & 27 & 12 & 5 & 7 & 2,8 \\
\hline (4) Unterstützung von benachteiligten Gebieten & 1 & 12 & 12 & 22 & 15 & 16 & 2,9 \\
\hline (5) andere Rollen & 7 & 6 & 7 & 7 & 6 & 44 & \\
\hline
\end{tabular}

Quelle: European Commission, op. cit.

\subsection{Dienstleistungsportfolio von Impulszentren}

Die nachstehende Tabelle 32 gibt eine Übersicht über die von Impulszentren angebotenen Dienstleistungen. Daraus lassen sich mehrere Beobachtungen ableiten: Das Spektrum ist breit, die nach innen gerichteten Dienstleistungen halten sich mit den außen gerichteten im Umfang die Waage, sind aber im jeweiligen Profil eher verschieden als gleichartig. Insgesamt lässt sich aber sagen, und darin unterscheidet sich die Mehrzahl der österreichischen Impulszentren nicht vom internationalen Geschehen, dass Impulszentren keineswegs, ja nicht einmal stark bevorzugt für die Mieter da sind, sondern für einen breiteren Kreis von Unternehmen auch außerhalb des Zentrums. 
Tabelle 32 Dienstleistungen von Impulszentren

\begin{tabular}{|l|r|r|r|r|r|}
\hline \multirow{2}{*}{ Dienstleistung } & \multicolumn{2}{c|}{ in-house } & \multicolumn{2}{c|}{ extern } & \multicolumn{2}{c|}{ gesamt } \\
\cline { 2 - 5 } & Zahl & {$[\%]$} & Zahl & {$[\%]$} & Zahl \\
\hline (1) Unterstützung bei Finanzierung (Banken, Förderungen, VC) & 68 & $89 \%$ & 28 & $37 \%$ & 96 \\
\hline (2) Networking, z.B. zu anderen Unternehmen, Kunden & 64 & $84 \%$ & 24 & $32 \%$ & 88 \\
\hline (3) Erstellung von Businessplänen & 62 & $82 \%$ & 25 & $33 \%$ & 87 \\
\hline (4) Managementtraining & 36 & $47 \%$ & 47 & $62 \%$ & 83 \\
\hline (5) Marktforschung, Verkauf und Marketing & 31 & $41 \%$ & 52 & $68 \%$ & 83 \\
\hline (6) Prä-Inkubations-Dienstleistungen & 66 & $87 \%$ & 15 & $20 \%$ & 81 \\
\hline (7) Unterstützung bei Entwicklung neuer Produkten / & 43 & $57 \%$ & 35 & $46 \%$ & 78 \\
Dienstleistungen & & & & & \\
\hline (8) Unterstützung bei e-business und anderen ICT-Angelegenheiten & 39 & $51 \%$ & 35 & $46 \%$ & 74 \\
\hline (9) Buchhaltung, Rechtsberatung, verwandte Dienstleistungen & 16 & $21 \%$ & 57 & $75 \%$ & 73 \\
\hline (10) Unterstützung bei Export und Partnersuche & 28 & $37 \%$ & 42 & $55 \%$ & 70 \\
\hline (11) Unterstützung bei Personalrekrutierung, Personalwesen & 32 & $42 \%$ & 35 & $46 \%$ & 67 \\
\hline (12) Mentoring, Übernahme von Aufsichtsratstätigkeit, Beratung & 38 & $50 \%$ & 27 & $36 \%$ & 65 \\
\hline (13) Inkubator VC, Business Angels & 31 & $41 \%$ & 32 & $42 \%$ & 63 \\
\hline (14) andere Dienstleistungen & 8 & $11 \%$ & 3 & $4 \%$ & 11 \\
\hline Gesamt & 562 & & 457 & & 1.019 \\
\hline
\end{tabular}

Quelle: European Commission, op. cit., befragt wurden 76 Zentren, darauf sind die Prozentangaben bezogen

\subsection{Errichtungs- und Betriebskosten und deren Finanzierung, Erreichung des Break Even}

Die Erhebung zeigt, dass die Durchschnittskosten für die Errichtung eines Impulszentrums bei 3,7 Mio. EUR liegen, im Median sind es gar nur 1,9 Mio. EUR. Noch interessanter als die Kosten ist deren Finanzierung. Hier zeigt sich, dass immerhin ein gutes Fünftel (22\%) der Finanzierung von der EU stammt, knapp die Hälfte (46\%) aus nationalen (und darin eingeschlossen auch regionalen und lokalen) öffentlichen Quellen, der private Sektor ist mit einem Achtel (13\%) vertreten.

Tabelle 33 Finanzierung der Errichtungskosten von Impulszentren

\begin{tabular}{|l|r|}
\hline \multicolumn{1}{|c|}{ Finanzierungsquelle } & \multicolumn{1}{|c|}{$\%$} \\
\hline (1) Förderungen: EU und andere internationale Fördereinrichtungen & 22 \\
\hline (2) Förderungen: nationale, regionale, lokale Fördereinrichtungen & 46 \\
\hline (3) Banken und andere private Unternehmen / Organisation & 13 \\
\hline (4) Universitäten Forschungseinrichtungen & 5 \\
\hline (5) andere Quellen & 13 \\
\hline Gesamt & 100 \\
\hline
\end{tabular}

Quelle: European Commission, op. cit.

Betreffend die Betriebskosten zeigt die Umfrage, dass diese im Durchschnitt 500.000 EUR im Jahr betragen, der Medianwert beträgt 300.000 EUR. Davon nehmen 41\% die Personalkosten ein, 22,1\% Umbau, Erhaltung und Erneuerung der Bausubstanz, Dienstleistungen an die Mieter 24,6\% sowie 13,3\% für Betriebskosten.

Dieser Wert dient natürlich nur als Richtschnur. Er ist aber hilfreich für die Positionierung der in dieser Studie vorgenommenen Evaluierung der RIF 2000 Förderung. Daraus wird ersichtlich, dass die durchschnittlichen Projektgrößen durchaus das reguläre Budget eines ganzen Betriebsjahres überschreiten können. Also handelt es sich um durchaus veritable Interventionen. Damit ergibt sich ein fließender Übergang zur Frage nach der Finanzierung der Betriebskosten, die im internationalen Vergleich 
aus Tabelle 34 ersichtlich wird. Erstaunlich ist hier der eher niedrige Anteil von Mieterträgen, was offensichtlich darauf zurückzuführen ist, dass begünstigte Mieten nicht als Förderungen dargestellt werden. Im gleichen Ausmaß wie Miteinnahmen erzielen die Zentren Einnahmen aus Förderungen.

Tabelle 34 Finanzierung der Betriebskosten von Impulszentren

\begin{tabular}{|l|r|}
\hline \multicolumn{1}{|c|}{ Finanzierungsquelle } & \multicolumn{1}{|c|}{$\%$} \\
\hline (1) Förderungen: EU und andere internationale Fördereinrichtungen & 10,1 \\
\hline (2) Förderungen: nationale, regionale, lokale Fördereinrichtungen & 27,3 \\
\hline (3) Banken und andere private Unternehmen / Organisation & 2,6 \\
\hline (4) Universitäten und Forschungseinrichtungen & 3,0 \\
\hline (5) Mieterträge & 39,5 \\
\hline (6) andere Erträge, z.B. aus Dienstleistungen & 11,1 \\
\hline (7) Einkommen aus Investitionen, z.B. aus Lizenzen oder Beteiligungen & 0,8 \\
\hline (8) andere Quellen & 5,6 \\
\hline Gesamt & 100,0 \\
\hline
\end{tabular}

Quelle: European Commission, op. cit.

Angesichts dieser Einkommensstruktur stellt sich die Frage nach dem Break Even. Hier zeigt sich ein erstaunliches Phänomen: Nur 40,8\% der Zentren haben das Erreichen eines Break Even innerhalb eines bestimmten Zeitraums als Gegenstand eines Geschäftsplans, bei 49,6\% der Zentren gibt es eine derartige Festlegung nicht, bei 9,6\% ist es unklar. Von denen, die ein diesbezügliches Ziel haben, wollen 60\% es innerhalb von 5 Jahren erreichen, 40\% brauchen länger ${ }^{70}$. Immerhin 29,6\% wollen ihr Break Even innerhalb von 2-3 Jahren erreicht haben. Überflüssig zu sagen, dass sich eine zu kurze Zeitspanne negativ auf das Akquisitionsverhalten auswirken kann, weil hier notgedrungen eine Strategie des First-come-first-serve zur Anwendung kommt und damit eine geringe Selektionswirkung auf die Auswahl der Mieter und in der Folge geringeren Synergieeffekten.

\subsection{Abhängigkeit von öffentlicher Finanzierung}

76,9\% der Impulszentren haben gemeinnützigen Status, die Hälfte (49,6\%) hat kein explizites Ziel der Erreichung eines Break Even. Es stellt sich also die Frage nach ihrer Abhängigkeit von öffentlicher Finanzierung. Tabelle 35 zeigt, dass bei ca. $60 \%$ die Beendigung der öffentlichen Finanzierung / Förderung zu einer deutlichen Reduzierung der Tätigkeiten der Zentren führen würde.

Tabelle 35 Konsequenzen des Rückzugs aus der öffentlichen Finanzierung von Impulszentren

\begin{tabular}{|l|r|r|}
\hline \multicolumn{1}{|c|}{ Bedeutung von öffentlicher Finanzierung } & Zahl & \multicolumn{1}{|c|}{ Anteil [\%] } \\
\hline (1) Aktivitäten würden auf dem gegenwärtigen Niveau beibehalten werden & 6 & 6,7 \\
\hline (2) Aktivitäten würden signifikant reduziert & 31 & 39,7 \\
\hline (3) Aktivitäten würden beendet & 17 & 21,8 \\
\hline (4) ohne Bedeutung, Zentrum erhält keine öffentliche Finanzierung & 9 & 11,5 \\
\hline (5) k.A. & 15 & 19,2 \\
\hline Gesamt & 78 & 100,0 \\
\hline
\end{tabular}

Quelle: European Commission, op. cit.

70 Es ist methodisch nicht ganz klar, was mit 'Break Even' und ähnlichen Indikatoren genau gemeint war. Für bestimmte Fragen ist das auch nicht so wichtig. Für unsere Analyse ist es dagegen aufschlussreich, zu sehen, dass es ein weites Spektrum an Positionen gibt. 
Die Tatsache, dass eines von fünf Zentren nicht weiß, was das Wegbrechen ihrer maßgeblichen Finanzierung für Folgen nach sich ziehen würde, ist auch ein Hinweise darauf, dass durchaus auch 'gewurstelt' wird und dass Produktivitätsreserven vorhanden sind.

\subsection{Sind die österreichischen Impulszentren anders als die ausländischen Inkubatoren?}

Im Grunde sind sie es nicht. Sie sind in etwa gleich groß, sie weisen eine ebenso breite Dienstleistungspalette auf, und sie bieten ihre Dienstleistungen nicht nur den Mietern, sondern auch Externen in ungemindertem Ausmaß an. Die öffentliche Hand als maßgeblicher Finanzier und die gemischte Finanzierung sind nicht unähnlich. Auch gibt es durch unklare Zielvorstellungen einerseits und durch Überfrachtung mit unterschiedlichen Erwartungen auch gehörige Produktivitätsreserven.

Positiv zu vermerken ist die Tatsache, dass doch die meisten österreichischen Impulszentren integriert sind in weiterreichende Strategien, zu denen nicht zuletzt das RIF 2000 einzurechnen ist. Weiters, dass in den meisten Fällen eine gemischt öffentlich-private Trägerschaft vorherrscht.

Das Vorhandensein von klaren und über die Zeit konsistenten Geschäftsplänen ist eher die Ausnahme, desgleichen und damit zusammen hängend, Unklarheit darüber, welchen Geschäftsmodellen die jeweiligen Zentren folgen. Dies wäre übrigens eine nicht unwesentliche Herausforderung für künftige Programme (vgl. dazu Kapitel 9, insb. 9.5), aber auch für die gegenwärtig laufenden Aktivitäten des Verbandes der Technologiezentren Österreichs (VTO). Ziel dieser horizontalen Bemühungen sollte es sein, den Anteil der öffentlichen Finanzierung so weit wie möglich zu minimieren und die Anreize für private Investoren klarer heraus zu arbeiten.

Es gibt eine gewisse anekdotische Evidenz darüber, dass private Zentren effizienter geführt werden. Es wäre allerdings vorschnell, Zentren in privates Eigentum überführen zu wollen. Vielmehr geht es darum, auf Basis expliziter Zielvereinbarungen die unterschiedlichen Anforderungen an das Zentrum abzuklären: Break Even, Auslastung bzw. Mieterlöse, Erlöse aus anderen Tätigkeiten, Dienstleistung für Mieter und Externe, bessere Beherrschung des Zielkonflikts hohe Mieterlöse vs. Wachstum und damit ein 'Hinauswachsen' aus dem Zentrum etc.. Hier kommen die Stakeholder ins Spiel, die, gemeinsam mit dem Management, entsprechende Vereinbarungen treffen müssen, die für die Beteiligten auch anreizwirksam sein müssen.

Ähnlich wie im internationalen Vergleich dominieren auch in Österreich Neubauten. Die Revitalisierung von Gebäuden ist die Ausnahme. Angesichts eines großen Leerstandes an Industrieimmobilien ist dies eigentlich ein beklagenswerter Zustand umso mehr als die immateriellen Leistungen (vgl. Tabelle 26 für Österreich, Tabelle 32 für den internationalen Vergleich) langfristig den höheren Stellenwert haben werden. Diese Leistungen sind in einem langfristigen Szenario auch durch Entgelte zu entlohnen, ohne dabei den privaten Dienstleistungssektor auszuhöhlen. Gegenwärtig herrscht eher die umgekehrte Richtung vor, insofern die Unternehmen mit einer Vielzahl von Dienstleistungen 'verwöhnt' werden, die im Rahmen der unterschied- 
lichsten Förderprojekte (vgl. Tabelle 23) angeboten werden. Für den langfristigen Bestand der Impulszentren, vor allem deren Management ist es wichtig - und dies wird im Prinzip auch schon praktiziert -, dass ein 'hinaus gewachsenes' Unternehmen weiterhin mit dem Zentrum verbunden bleibt, nicht zuletzt weil es in der Gründungsund Aufbauphase gut betreut wurde ${ }^{71}$ und nunmehr weiterhin die Dienstleistungen des Zentrums in Anspruch nimmt, in jedem Fall aber unter starker Einbindung des privaten Dienstleistungssektors.

Schließlich und endlich ist das Managementteam, dessen Fertigkeiten und Netzwerke, der entscheidende Faktor für die Leistungsfähigkeit von Impulszentren. Erst danach kommen das Einzugsgebiet, die Wirtschaftsstruktur und andere 'vorgefundene' Faktoren. Für die Eigentümer, Investoren, und sonstige Aufsichts- und Beratungsorgane sind daher die 'Führung dieser Führungspersonen' das Zentrum ihrer Aufmerksamkeit (vgl. Kapitel 9.3).

71 Man erkennt hier ein sehr wirkungsvolles Regulativ. 


\section{Die Zukunft des RIF 2000 Programms}

Seit dem Anlaufen des RIF 2000 Programms hat sich vieles geändert (Tabelle 6, Kapitel 2.4). Die Zentren sind vernetzt - untereinander, mit anderen (öffentlichen) Institutionen (Kapitel 5.3). Ihr Dienstleistungsportfolio ist über weite Strecken komplett und das Netz der Zentren über Österreich ist dicht besetzt (Kapitel 5.4). Es gibt wohl kein Unternehmen in Österreich, das nicht nach einer einstündigen Fahrt mit dem Auto ein Zentrum erreichen würde. Die Zentren sind erfolgreiche Teilnehmer im Markt der öffentlich geförderten Projekte (Kapitel 5.3). Sie bieten ihre Dienstleistungen in der Regel sowohl für ihre eingemieteten Unternehmen als auch für externe Firmen an und dort, wo sie selbst keine Kompetenzen aufweisen, sind sie Vereinbarungen mit Dritten eingegangen (Kapitel 5.4). Tausende Gemeinden, Städte, Landesentwicklungsagenturen, Wirtschaftskammern, HTLs, Universitäten, Fachhochschulen, Forschungseinrichtungen sind über Projekte vernetzt. Über die eingegangenen Beteiligungen an INTERREG-Projekten gibt es eine veritable grenzüberschreitende Vernetzung - zur Nachbarregion jenseits der Staatsgrenze, aber auch zu Regionen, die 1.000 und mehr Kilometer entfernt sind.

So gesehen finden wir eine pulsierende, unternehmerische, Chancen suchende und von überdurchschnittlich motivierten Menschen geprägte Szene vor. Viele Zentren bzw. Zentrumsmanager haben sich zu regionalen Entwicklungsagenturen transformiert, die eine breite Palette von Bedürfnissen bedienen. Dies alles lässt sich durch Indikatoren nachweisen, und in der hier vorliegenden Analyse ist vieles davon abgebildet.

Auf der anderen Seite und bei genauerem Hinsehen kommen wir zu einem ganz anderen Bild, und am Ende handelt es sich um ein Vexierbild, welches je nach Perspektive kippt: Der Sektor ist mit Förderangeboten überhitzt, die Anforderungen an das Management von Seiten der jeweiligen Gesellschafter sind vielfach unerfüllbar, weil zu divergierend und überzogen, die finanzielle Situation der Zentren ist oftmals prekär, die Manager operieren am Rande der Selbstausbeutung, die eigentliche wird Mission vernachlässigt.

Beide Bilder treffen zu und die Auflösung des vermeintlichen Widerspruchs liegt in einer dynamischen Betrachtung. Es sind seit Einführung des RIF 2000 Programms nur vier Jahre vergangen. Das Angebot an Fördermöglichkeiten, auch die Verlockungen sind paradiesisch. Die Gesellschafter der Zentren waren gleichermaßen beflügelt und daher in Bezug auf das Management und deren Ziele und Präferenzen wenig diskriminierend und fokussiert. So gesehen, wurde viel begonnen und wenig zu Ende geführt. Es wurde viel gesät, aber wenig gejätet und ausgedünnt, geschweige denn geerntet. $^{72}$

\subsection{RIF 2000 Programm hat seine Mission erfüllt}

Die Zentren sind mobilisiert. Ihre Außenorientierung, ihre Vernetzungsarbeit, die Zahl der Plattformen und Drehscheiben, die Initiativen und Events gehen in die hun-

72 Wohlgemerkt, wir sprechen hier nicht primär von den REGplus- und RIFupgrade Projekten, sondern von der großen Zahl an Projekten aus den unterschiedlichsten Förderprogrammen. Vgl. auch Tabelle 23. 
dert und tausend. Das Netzwerk der Zentren ist dicht und es bedarf keiner Erweiterung auch nur irgend einer Funktion. Viele Zentren haben sich in kleine Regionalentwicklungsagenturen verwandelt und dienen als Anlaufstelle für Unternehmen. Für die Bürgermeister sind sie 'ihr' Organ. Die Zielsetzungen des RIF 2000 Programms sind über weite Strecken erfüllt, ja übererfüllt. Dort, wo es noch des einen oder anderen Projektes bedürfte, kann man sich anderer existierender Angebote bedienen ${ }^{73}$.

\subsection{Kargheit und Fokussierung}

Die Kooperationen müssen auf den Prüfstand, vor allem jene, die durch Förderprogramme zustande gekommen sind. Sie müssen sich nun im Regelbetrieb bewähren. Der Regelbetrieb ist in erster Instanz durch die Bedienung der beiden alten und unvermindert neuen Funktionen bestimmt: (i) Brutkasten, also höhere Überlebensquote der jungen Unternehmen, (ii) Treibhaus, d.h. rascheres Wachstum bei den Unternehmen im Zentrum. Es haben sich in den vergangenen Jahren zahlreiche zusätzliche Aufgaben hinzugesellt. Sie stehen häufig im Verdacht, dass sie gelegenheitsgetrieben und vielfach nicht durch eine gründliche Analyse der regionalen Situation begründet sind. Wir wollen damit ausdrücken, dass die Impulszentren durchaus regionalwirtschaftliche Aufgaben übernehmen sollen. Welche Leistungen dies sind, hängt aber ganz wesentlich vom Kontext ab, also von der Politik des jeweiligen Bundeslandes, dem Spielraum, den die Gesellschafter zulassen und dann wohl auch Verantwortung und (politische) Haftung unternehmen, nicht zuletzt und vor allem von den ökonomischen und institutionellen Gegebenheiten in der Region. Wichtig ist hier, das systemimmanente Konflikte angesprochen und gelöst werden. Ein typischer Konflikt besteht darin, dass durchschnittliche Zentrumsmanager kein Interesse an jener Art von Wachstum ihrer Mieter haben, das dazu führt, dass diese das Zentrum - wachstumsbedingt - verlassen. Eine anderer Konflikt ist die Flucht in die Vernetzung und die Regionalentwicklung unter Preisgabe der Sorge um die Immobilie und die Mieter. Benchmark und Stachel im Fleisch der Vernetzungs-, Event- und Initiativkultur ist die Tatsache, dass sich Zentren privatwirtschaftlich betreiben lassen.

\subsection{Das Humanpotenzial nutzen}

Das RIF 2000 Programm hat nicht unwesentlich dazu beigetragen, dass einige Dutzend Zentrumsmanager, wenn sie sich schon nicht am Projekt empor gearbeitet haben, so doch entdeckt wurden. Sei es, dass sie erfolgreich Projekte eingeworben haben, die ihnen einen Durchbruch in ihrer Region ermöglicht haben, sei es, dass im Zuge des Projekts - und in aller Regel auch anderer Projekte - ein Beziehungsnetzwerk aufgebaut wurde, sei es schließlich, dass die Mitarbeiter am Projekt gelernt haben. Wir sehen in den Managern der Zentren, ihren Schlüsselmitarbeitern und ihren Netzwerken zu den Unternehmen und sonstigen Akteuren in der Region, nicht zuletzt zur Entwicklungsagentur des Landes und zur Förderabwicklungsstelle aufgebaute Erfahrung als das wertvollste Ergebnis an. Allerdings verflüchtigen sich diese Potenziale auch rasch, wenn sie nicht bewirtschaftet werden. Es steht zu fürchten, dass die

73 Es gibt vielmehr bei den Projekten zunehmend Überlappungen und Rosinenpicken. Nicht ganz zufällig ist verschiedentlich der Ausdruck 'satte Förderquote' aufgetaucht. Zur Kontrastierung ein Entwicklungsprogramm des Bundeslandes, aus der Sicht eines Teilnehmers: "Das [Programm] ist ein Problem für mich. Ich habe zwei Projekte eingereicht. Das ist mit so vielen Auflagen verbunden, dass einige Partner gesagt haben, bei der Förderquote machen wir das nicht. Die Förderquote bei [Programm] beträgt 40\%. Es gibt ganz wenige anrechenbare Kosten. Ich als Geschäftsführer kann mich gar nicht einrechnen." 
meisten Beziehungen dieses Schicksal auch tatsächlich erleiden - unter anderem und paradoxerweise dadurch, dass neue Projekte eingeworben werden. So liegt es an den Managern, den Gesellschaftern der Zentren und den jeweiligen Landesstellen, allen voran den Entwicklungsagenturen, rasch und beherzt die aufgebauten Potenziale zu realisieren und nachhaltig zu verankern. Die nahe liegende Ebene der Umsetzung ist abermals die Kernaufgabe der Zentren: Gründung, Wachstum, Entwicklung, Vernetzung. Wichtigster Aktionspunkt ist hier die Aufklärung der Gesellschafter bzw. jene Akteure, die an normalen Tagen Regionalentwicklung und Vernetzung preisen und zweimal im Jahr auf Auslastung und EGT beharren.

Wir werden weiter unten Vorschläge machen, inwieweit dieses in den Zentrumsmanagern aufgebaute Potenzial in Zukunft systematisch genutzt werden kann.

\subsection{Hohe Anerkennung der Förderabwicklungsstelle und ihrer Mitarbeiter, veränderte Rolle in Zukunft}

Wenn von Humanpotenzial und Erfahrung die Rede ist, müssen auch die Förderabwicklungsstelle und im Speziellen die mit der Abwicklung des REGplus Programms befassten Mitarbeiter erwähnt werden. Ihre Arbeit wurde - ausnahmslos - als kooperativ, transparent, fair und effizient empfunden. Der administrative und Kontrollaufwand wurde als angemessen empfunden und es gab im Grunde nicht nur keine ernsthafte Kritik, sondern durchwegs Lob für die Mitarbeiter der Abwicklungsstelle. Besonders der Balanceakt zwischen verbindlicher und nützlicher Information im Vorfeld von Ausschreibungen einerseits und der gebotenen Neutralität andererseits wurde gewürdigt. Nicht zuletzt ist der Vorort-Besuch als hilfreich und vertrauensbildend gewürdigt worden. Im Besonderen konnten im Zuge dieser Gespräche, Ziele und Erwartungen präzisiert und fallweise auch angepasst werden.

Für künftige einschlägige Programme sollten derartige Besuche nicht nur den Charakter eines Reviews, sondern auch den einer - in Grenzen - möglichen Anpassung des Projekts haben. An der Kompetenz und der Vertrauenswürdigkeit der Mitarbeiter der FFG sollte es nach den Befunden aus dieser Evaluierung nicht mangeln. Wir leiten aus diesen Befunden, die sich durch das transparente und kooperative Verhalten auch gegenüber den Evaluatoren bestätigen lassen, eine neue und reichere Rollendefinition für die Mitarbeiter der Förderagentur ab: Nachdem wesentliche Bewertungen und Entscheidungen nicht von den Mitarbeitern der Förderagentur selbst vorgenommen werden, sondern de facto durch eine unabhängige Jury, und weil überdies die Verlässlichkeit und Akzeptanz der Förderagentur bzw. ihrer Mitarbeiter im Rahmen dieser Evaluierung außer Zweifel gestellt wurde, ist es nicht nur möglich, sondern auch sinnvoll, das Rollenprofil der Förderagentur um die Aufgabe des Coachings zu erweitern. Coaching heißt hier, mit Hilfe des Projekts dazu beizutragen, dass sich die Organisation, hier das Impulszentrum, ihrer Mission und ihren spezifischen Aufgaben gemäß weiterentwickelt. Im Einzelnen erfordert dies ein klares Rollenverständnis auf Seiten der Förderagentur und ein höherer Ressourceneinsatz. Ersteres ist gegeben, zweiteres steht noch auf unsicheren Beinen, weil gegenwärtig die Reduktion der administrativen Kosten der FFG diskutiert wird. Durch Übergang zu größeren Projekten und damit der Zahl der Projekte sollte diese Situation entschärft werden können, unbeschadet der Sinnhaftigkeit der vermehrten Coachings. 


\subsection{Ein neues Programm?}

Definitiv kein neues Programm, das exklusiv an Impulszentren gerichtet ist. Aber es gibt Zentren, die schon mehr lokale Entwicklungsagentur sind als Gründer- und Technologiezentrum. Es gibt auf der anderen Seite Zentrumsmanager, die das Zeug für Größeres haben und denen man zusätzliche bzw. größere Aufgaben übertragen kann. Schließlich überlagern sich bereits diverse Strukturprogramme: Adressaten von RIF 2000 sind gleichzeitig Empfänger von Förderungen aus dem protec Programm und überdies Zielgruppe des CIR-CE Programms. Auch die Berührung mit den Kompetenzzentrenprogrammen sowie dem AplusB Programm ist fallweise gegeben. Schließlich sind Impulszentren bevorzugte Adressaten von Landesförderungen, die ihrerseits aus Programmen der EU ko-finanziert werden.

\section{Übergang von 'Vielfalt' zu 'Selektion'}

Es gibt ferner Zentren bzw. Manager, die ein extensives Szenario verfolgen und auch realisieren, andere haben erhebliche Schwierigkeiten, auch nur eine Halbtagskraft anzustellen. Ob man diesen ein Projekt bzw. eine Aufgabe übertragen soll, hängt in hohem Maß davon ab, welche Managementkapazität, Vernetzung und Sichtbarkeit das Zentrum bzw. dessen Manager hat. Dies ist mindestens so wichtig wie die spezifische Eignung der Projekts an sich. Es gibt überdies so viele Zentren, dass eine Reduktion der Zahl der Zentren durchaus verkraftbar ist, zumal dann, wenn man bedenkt, dass nicht selten Zentren weniger nach dem, wenn überhaupt, erhobenen Bedarf, sondern nach eher kontingenten Gesichtspunkten eingerichtet wurden. Aus diesen Gründen kann die Phase, in der 'Vielfalt' das Leitkonzept war, nunmehr durch die Phase 'Selektion' abgelöst werden. Die wesentlichen Charakteristika eines solchen Programms sind die folgenden:

\section{Offenere Zielgruppe}

Da sich die Grenzen zwischen unterschiedlichen intermediären Institutionen zunehmend verschieben, sollte aus der Not eine Tugend gemacht werden, dass gefördert werden soll, was an intermediären Leistungen wünschenswert und sinnvoll ist. Logisch zweitrangig ist dabei die Institution selbst. Ein Gang allein durch die RIF 2000 Projekte liefert einen guten Eindruck, was dies alles heißen kann - aber keineswegs heißen muss. An Phantasie mangelt es jedenfalls nicht.

\section{Primärer Fördergegenstand ist die Organisation, nicht das Projekt}

Das Projekt dient als Mittel zum Zweck der Entwicklung der Organisation zur Erfüllung ihrer Mission. Worauf es ankommt, ist, dass sich mit Hilfe des Projekts die Organisation weiterentwickelt kann. Dies bedeutet, dass im Zuge der Bewertung des Fördervorhabens die Charakteristika der Organisation und ihrer leitenden Mitarbeiter wichtiger sind als die Charakteristika des Projekts. Darin eingeschlossen ist insbesondere das Portfolio der unterschiedlichen Kompetenzen, Dienstleistungen und (Förder)Projekte.

\section{Begutachtung der Fördervorhaben durch eine unabhängige Jury und Coaching der geförderten Organisation durch die Förderagentur}

Die Begutachtung der Fördervorhaben durch eine unabhängige Jury hat sich durchwegs als zweckmäßig erwiesen. Einem spezifischen Schwachpunkt in der Vergan- 
genheit sollte in Zukunft durch erhöhte Aufmerksamkeit begegnet werden, nämlich der deutlich stärkeren Beachtung der werbenden Organisation und ihres Kontexts. Wir halten diese Anforderung für einlösbar, weil es mehr um die Anerkennung der höheren Bedeutung dieses Kriteriums geht als um dessen praktischer Handhabung.

Auf Seiten der Förderagentur wird zum nunmehr tragenden Prinzip erhoben, was in Ansätzen schon stattgefunden hat stattfindet, allerdings implizit und unbemerkt, nämlich das Coaching des Förderempfängers durch die Förderagentur. Die Rollenprobleme sollten in diesem Ansatz beherrschbar sein. Erstens hat die FFG ein hohes $\mathrm{Maß}$ an Fairness und Transparenz bewiesen und zweitens verfolgen beide dasselbe Ziel, nämlich die Leistungsfähigkeit der Organisation zu erhöhen.

\section{Übergang zu größeren Projekten}

Dieses Kriterium ergibt sich indirekt aus den vorgenannten Kriterien. Der Aufwand erhöht sich naturgemäß, sowohl bei den Antragstellern als auch bei Jury und Förderagentur. Dazu kommt, dass sich die werbenden Organisationen - zurecht - die Frage stellen werden, warum nunmehr alle Karten offen gelegt werden müssen.

Dieser Übergang ist nur rechtfertigbar, wenn es sich um größere Vorhaben handelt. Ein Übergang zu größeren Projekten würde dem Prinzip 'Selektion' unmittelbar entgegenkommen, darüber hinaus dazu beitragen, dass sich Spreu und Weizen trennen und es zu kritischen Größen bei den geförderten Organisationen kommt ${ }^{74}$.

\section{Coaching als Förderprinzip}

Die größeren und vermutlich auch längeren Projekte (ein weiches Kriterium), das heterogene Umfeld der Projekte und schließlich die Orientierung auf die Trägerorganisation statt auf die Umsetzung des Projekts macht eine aktivere und flexiblere Betreuung seitens der Förderagentur notwendig. Das Rollenverständnis und die 'moralische' als auch professionelle Qualifikation sind soweit vorhanden, dass mit einem derartigen Ansatz begonnen werden kann.

\section{Fusion / Abstimmung mit anderen Programmen und Politikebenen}

Auf Bundesebene gibt es, wie mehrfach erwähnt, eine Handvoll Programme, die in mehrerer Hinsicht ähnlich sind. Im Besonderen stellen sie auf Strukturen und damit explizit oder implizit auf intermediäre Organisationen ab. Es gibt zumindest ein Unbehagen mit dieser Situation und Versuche einer Verständigung, über Alternativen nachzudenken. Soweit die Bundesebene. Ort des Geschehens ist vor allem die FFG, bei genauerer Betrachtung zeigt sich auch der Bedarf eines Abgleichs mit dem AWS.

Eine zweite, ja dritte Politikebene ist aber nicht weniger betroffen. Impulszentren haben sich, vor allem in den vergangen 5-8 Jahren als wesentliche Träger und Adressaten der Wirtschafts- und Innovationspolitik auf Landesebene etabliert, im Einzelnen sind auch noch Städte und Gemeinden bzw. Gemeindeverbände angesprochen. Hier gibt es im Verhältnis Bund-Bundesländer sowohl Synergien und gute Zusammenarbeit als auch zahlreiche Reibungsflächen. Ein stärkeres Eingehen auf die

74 Es macht im Gegenzug überhaupt keine Mühe, im Zuge der Begutachtung auch ein Kriterium einzubauen, dass vor überdimensionierten Organisationen schützt. 
spezifischen Gegebenheiten der Länder und Gemeinden in Bezug auf deren Politik, aber auch auf die jeweilige Ausstattung der förderungswerbenden Organisationen eröffnet die Möglichkeit, mehr Synergien zu lukrieren, die eine oder andere Konfliktlinie zu entschärfen und insgesamt mehr zum Verstehen der jeweils spezifischen Situation beizutragen. Dies ist nämlich eine der positivsten Beobachtungen, die sowohl bei der Förderlinie REGplus als auch bei RIFupgrade gemacht werden konnte, nämlich das sachorientierte und kooperative Rollenverständnis in der praktischen Abwicklung des Programms bzw. der einzelnen Projekte.

\subsection{Checkliste für künftige Programmplanung aufgrund der Erfahrung mit RIF 2000: To whom it may concern!}

Wir schließen dieses Kapital über die Zukunft des RIF 2000 Programms mit einer Übersicht (Tabelle 36) ab, in der Kriterien aufgelistet sind, die für die Planung künftiger Programme hilfreich sein können. Es sind über weite Strecken generische Empfehlungen, die für eine ganze Klasse von Programmen herangezogen werden können. Insofern heißt es im Untertitel "To whom it may concern!", analog der Gepflogenheit in Arbeitszeugnissen im anglo-amerikanischen Kulturkreis.

Tabelle 36 Checkliste für künftige Programmplanung aufgrund der Erfahrung mit RIF 2000

\begin{tabular}{|l|l|}
\hline \multicolumn{1}{|c|}{ Kriterium } & \multicolumn{1}{c|}{ Beschreibung } \\
\hline $\begin{array}{l}\text { begrenzte } \\
\text { Intervention }\end{array}$ & $\begin{array}{l}\text { Innerhalb von 5 Jahren lässt sich ein Programm entwickeln und auch } \\
\text { erfolgreich beenden. Also ist begrenzte Intervention möglich. }\end{array}$ \\
\hline $\begin{array}{l}\text { stärkere Berücksichti- } \\
\text { gung von Geschichte } \\
\text { und Kontext }\end{array}$ & $\begin{array}{l}\text { Während der Laufzeit eines Programms kann sich sein Kontext erheblich } \\
\text { verändern. Mehr denn je sind Geschichte und Kontext in das Design von } \\
\text { Programmen und in die Begründung von Projekten einzubeziehen. Bei einer } \\
\text { statischen Programmentwicklung besteht zudem die Gefahr, dass zuviel } \\
\text { (gestriger) Zeitgeist hineingepackt wird. Vernetzung und Kooperation sind } \\
\text { solche Beispiele. Daher die Möglichkeit, das Programm während der Laufzeit } \\
\text { zu ändern, von vornherein vorsehen. Ein geeignetes Mittel sind hier Aus- } \\
\text { schreibungen, die eine feste und eine variable Komponente enthalten können } \\
\text { und worin der variable Teil den jeweils aktuellen Anforderungen angepasst } \\
\text { werden kann. }\end{array}$ \\
\hline $\begin{array}{l}\text { Förderportfolio } \\
\text { stärker beachten - } \\
\text { Achtung vor } \\
\text { Verdrängung durch } \\
\text { Förderung }\end{array}$ & $\begin{array}{l}\text { Bei der Entwicklung von Programmen ist wesentlich auf die Kompatibilität } \\
\text { mit benachbarten Programmen zu achten. Es gibt insbesondere einen Wett- } \\
\text { bewerb von ähnlich gelagerten Förderprogrammen (vertikal, also Land - } \\
\text { Bund - EU, aber auch horizontal) und damit verbunden, Rosinenpicken bei } \\
\text { den Förderadressaten. Bei RIF 2000 sind zahlreiche Hinweise auf Verdrän- } \\
\text { gung der primären Mission durch Förderung aufgetaucht. }\end{array}$ \\
\hline $\begin{array}{l}\text { Metaziel: Vielfalt } \\
\text { oder Selektion }\end{array}$ & $\begin{array}{l}\text { Beim Design neuer Programme ist verstärkt auf das Portfolio verwandter } \\
\text { Programme zu achten. Die meisten Programmen zielen auf Vielfalt, selten } \\
\text { auf Selektion. Nach 5-8 fetten Jahren sollte dem Paradigma 'Vielfalt' } \\
\text { zunehmend mehr vom Paradigma 'Selektion' abgelöst werden. Dadurch ist es } \\
\text { besser möglich, Fragen der kritischen Massen, der Kontinuität und der } \\
\text { Nachhaltigkeit zu verfolgen. Dies macht es verstärkt erforderlich, die } \\
\text { geförderte Organisation zum Gegenstand der Förderentscheidung zu machen. } \\
\text { Dementsprechend sind auch die Bewertungskriterien anzupassen. }\end{array}$ \\
\hline
\end{tabular}




\begin{tabular}{|c|c|}
\hline \begin{tabular}{|l|} 
Dynamische \\
Zieldefinition
\end{tabular} & $\begin{array}{l}\text { Die verstärkte Berücksichtigung sowohl des Kontexts des gesamten Pro- } \\
\text { gramms als auch des Kontexts der jeweiligen Projekte und insbesondere der } \\
\text { Förderwerber bedingt eine offenere Zieldefinition. Dies bedingt auf der einen } \\
\text { Seite eine stärkere Abstimmung mit anderen Programmen (Inhalt, Zielgrup- } \\
\text { pen, Abwicklungsmodus, Termine, eingereichte Projekte etc.), auf der ande- } \\
\text { ren Seite erfordert es ein stärkeres Eingehen auf den Förderwerber, dessen } \\
\text { Geschäftsmodell, Geschichte, Kontext und Geschäftsportfolio, um, von dort } \\
\text { ausgehend, die Förderungswürdigkeit zu beurteilen. Primärer Fördergegen- } \\
\text { stand ist also die Organisation, nicht das Projekt. Dieses dient als Mittel zum } \\
\text { Zweck der Entwicklung der Organisation zur Erfüllung ihrer jeweiligen } \\
\text { Mission. }\end{array}$ \\
\hline $\begin{array}{l}\text { Sorgfältige Pflege des } \\
\text { Humankapitals }\end{array}$ & $\begin{array}{l}\text { Humankapital ist ein wesentlicher Erfolgsfaktor. Im Zuge von Förderpro- } \\
\text { grammen werden auch immer wieder 'gute Leute' entdeckt. Diese sind eine } \\
\text { bevorzugte Zielgruppe für weiterführende Aufgaben (u.a. im Rahmen anderer } \\
\text { Programme). Man findet sie sowohl unter den Förderempfängern als auch in } \\
\text { der Förderagentur. Sie zeichnen sich insbesondere durch unternehmerisches } \\
\text { Handeln und im Besonderen durch den Aufbau von Beziehungen und Zu- } \\
\text { gängen aus. Diese 'Ressourcen' sind flüchtig, man muss sie also rasch nützen. }\end{array}$ \\
\hline \begin{tabular}{|l} 
Coaching als \\
Förderprinzip
\end{tabular} & $\begin{array}{l}\text { Unter bestimmten Umständen ist es wichtig, mit einem Projekt zu beginnen, } \\
\text { allerdings die Möglichkeit vorzusehen, es während der Laufzeit an veränderte } \\
\text { Bedingungen anzupassen. Hierzu sind einige Bedingungen einzuhalten: (i) } \\
\text { eher größere Projekte, (ii) hohes gegenseitiges Vertrauen zwischen Förderer } \\
\text { und Geförderten, (iii) primäre Konzentration auf die geförderte Organisation } \\
\text { und nicht so sehr auf das geförderte Projekt, also Verwendung des geförder- } \\
\text { ten Projekts zum Zweck der Entwicklung der Gesamtorganisation. }\end{array}$ \\
\hline $\begin{array}{l}\text { Übergang zu } \\
\text { größeren Projekten }\end{array}$ & $\begin{array}{l}\text { Mit Hilfe von größeren Projekten können folgende Ziele besser verfolgt } \\
\text { werden: (i) besseres Eingehen auf den Förderwerber, dessen Portfolio und } \\
\text { Geschäftssituation, (ii) bessere Möglichkeit, zur Entwicklung des Förder- } \\
\text { werbers (mit Hilfe des geförderten Projektes) beizutragen, (iii) höhere Selek- } \\
\text { tionswirkung unter den Geförderten, (iv) leichterer Aufbau von kritischen } \\
\text { Massen und von Nachhaltigkeit. }\end{array}$ \\
\hline $\begin{array}{l}\text { Begutachtung der } \\
\text { Fördervorhaben } \\
\text { durch eine } \\
\text { unabhängige Jury }\end{array}$ & $\begin{array}{l}\text { Die Begutachtung der Fördervorhaben durch eine unabhängige Jury ist ein } \\
\text { robustes Konzept und erlaubt insbesondere den verstärkten Übergang in der } \\
\text { Rolle der Fördereinrichtung zur Ausübung ihrer Coaching-Aufgabe. Die } \\
\text { potenziellen Rollenkonflikte sollten in diesem Ansatz beherrschbar sein. }\end{array}$ \\
\hline $\begin{array}{l}\text { Fusion / Abstimmung } \\
\text { mit anderen } \\
\text { Programmen und } \\
\text { Politikebenen }\end{array}$ & $\begin{array}{l}\text { Auf Bundesebene gibt es eine Handvoll Programme, die in mehrfacher } \\
\text { Hinsicht ähnlich sind. Dazu kommen Programme der Länder und der EU. Die } \\
\text { Erfahrung, dass zahlreiche Organisationen bei mehreren, ja zahlreichen Pro- } \\
\text { grammen um Förderung einkommen, erzeugt eine spezifische Kontrolllücke. } \\
\text { Sie besteht darin, dass weder die Förderer noch die Geförderten große Auf- } \\
\text { merksamkeit den Portfolios der Projekte entgegen bringen (können). Gerade } \\
\text { Impulszentren haben in den vergangenen Jahren ein Verhalten entwickelt, } \\
\text { demzufolge sie sich zunehmend zu 'Eier legenden Wollmilchsäuen' ent- } \\
\text { wickeln, nicht zuletzt durch die gleichzeitige Inanspruchnahme unterschied- } \\
\text { licher Förderprogramme und der Übernahme unterschiedlicher Rollen. Dieser } \\
\text { Fall zeigt, dass es auch den Bedarf nach Politikkoordination gibt. Hier reicht } \\
\text { es nicht aus, beim Design von Programmen wechselseitige Konsultationen } \\
\text { vorzusehen. Vielmehr kann, selbst bei homogenem Verhalten des Bundes, } \\
\text { die Politik der Länder so verschieden sein, dass diese Unterschiedlichkeiten } \\
\text { nicht durch ein Konsultationsverfahren vor Verabschiedung der Förderricht- } \\
\text { linien aufgefangen werden kann. Weiterführende Koordinationen sind } \\
\text { notwendig. Die im Rahmen von RIF } 2000 \text { aufgebauten Beziehungen und } \\
\text { Vertrauenspotenziale zeigen eine aussichtsreiche Richtung an. }\end{array}$ \\
\hline
\end{tabular}

